\title{
Propositions
}

1. Metabolome analysis in plasma of the host can detect an effect of a compound on the gut microbiome.

(this thesis)

2. Post-puberty Wistar rats at the age of 10 weeks show no or negligible sex-specific effects, neither in the microbial community (the microgenderome) nor in the feces metabolome.

(this thesis)

3. Test strategies for potential genome damage from CRISPR/Cas9 gene editing are needed to clarify potential risks with the final goal, to facilitate and accelerate innovative research in the field of plant science, biobased chemicals or gene therapies in Europe.

4. Algorithms will provide artificial intelligence systems with computational narrative intelligence, which will transform them to human-like members of our society.

5. The cruelest and most merciless master teacher of our mind and attitude (to life) is our body.

6. Rhineland-Palatinate will lose their famous cultivation areas of Riesling grapes due to the climate change.

Propositions belonging to the thesis, entitled

'Influence of the gut microbiome on plasma metabolite patterns'.

Christina Behr

Wageningen, 15 February 2019. 


\section{Influence of the gut microbiome on plasma metabolite patterns}

Christina Behr 


\section{Influence of the gut microbiome on plasma metabolite patterns}

Christina Behr 


\section{Thesis committee}

\section{Promotors}

Prof. Dr Bennard van Ravenzwaay

Professor of Reproduction and Developmental Toxicology

Wageningen University \& Research

Senior Vice President of Experimental Toxicology and Ecology BASF SE, Ludwigshafen,

Germany

Prof. Dr Ivonne M.C.M. Rietjens

Professor of Toxicology

Wageningen University \& Research

\section{Co-Promotor}

Dr Karsten Beekmann

Assistant professor, Sub-department of Toxicology

Wageningen University \& Research

\section{Other members}

Prof. Dr Frederik-Jan van Schooten, Maastricht University

Prof. Dr Sander A.H Kersten, Wageningen University \& Research

Dr Ad A.C.M. Peijnenburg, Wageningen University \& Research

Dr Carsten Sieden, BASF SE, Ludwigshafen, Germany

This research was conducted under the auspices of the Graduate School VLAG (Advanced studies in Food Technology, Agrobiotechnology, Nutrition and Health Sciences). 


\title{
Influence of the gut microbiome on plasma metabolite patterns
}

\author{
Christina Behr
}

Thesis

submitted in fulfilment of the requirements for the degree of doctor

at Wageningen University

by the authority of the Rector Magnificus

Prof. Dr A.P.J. Mol,

in the presence of the

Thesis Committee appointed by the Academic Board

to be defended in public

on Friday 15 February 2019

at 1:30 p.m. in the Aula. 


\section{Christina Behr}

Influence of the gut microbiome on plasma metabolite patterns, 188 pages.

PhD thesis, Wageningen University, Wageningen, the Netherlands (2019)

With references, with summary in English

ISBN: 978-94-6343-378-5

DOI: https://doi.org/10.18174/463891 


\section{Table of Contents}

Chapter 1

General introduction

Chapter 2

Gut microbiome-related metabolic changes in plasma of antibiotic-treated rats

\section{Chapter 3}

Microbiome-related metabolite changes in gut tissue, cecum content and feces of rats treated with antibiotics

\section{Chapter 4}

Impact of lincosamides antibiotics on the composition of the rat gut microbiota and the metabolite profile of plasma and feces

\section{Chapter 5}

Analysis of metabolome changes in the bile acid pool in feces and plasma of antibiotic-treated rats

\section{Chapter 6}

General discussion

\section{Chapter 7}

Summary

\section{Appendix}

Acknowledgements, Curriculum Vitae, List of publications, Overview of completed training activities 


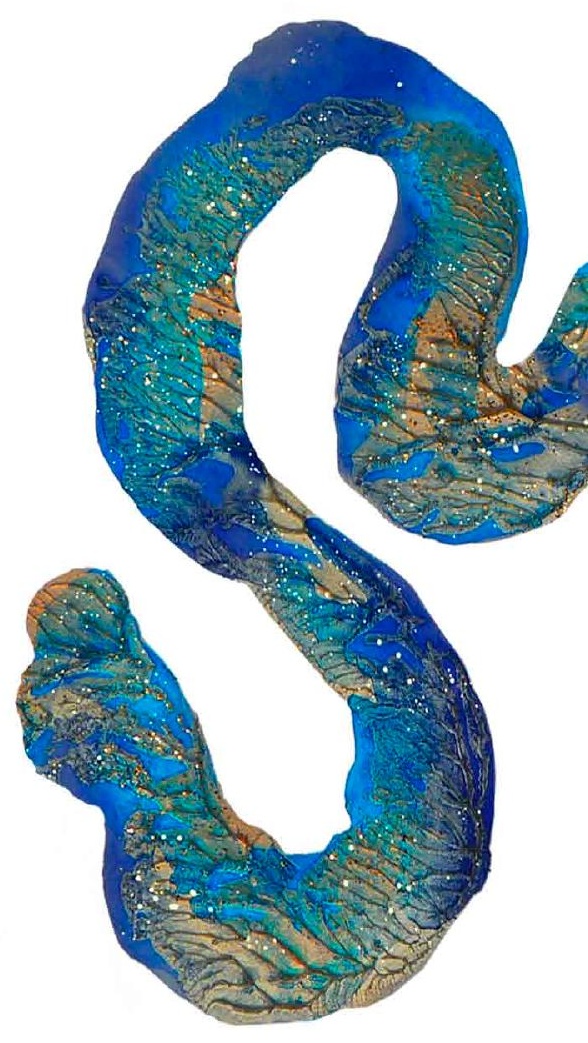




\section{Chapter 1}

General introduction

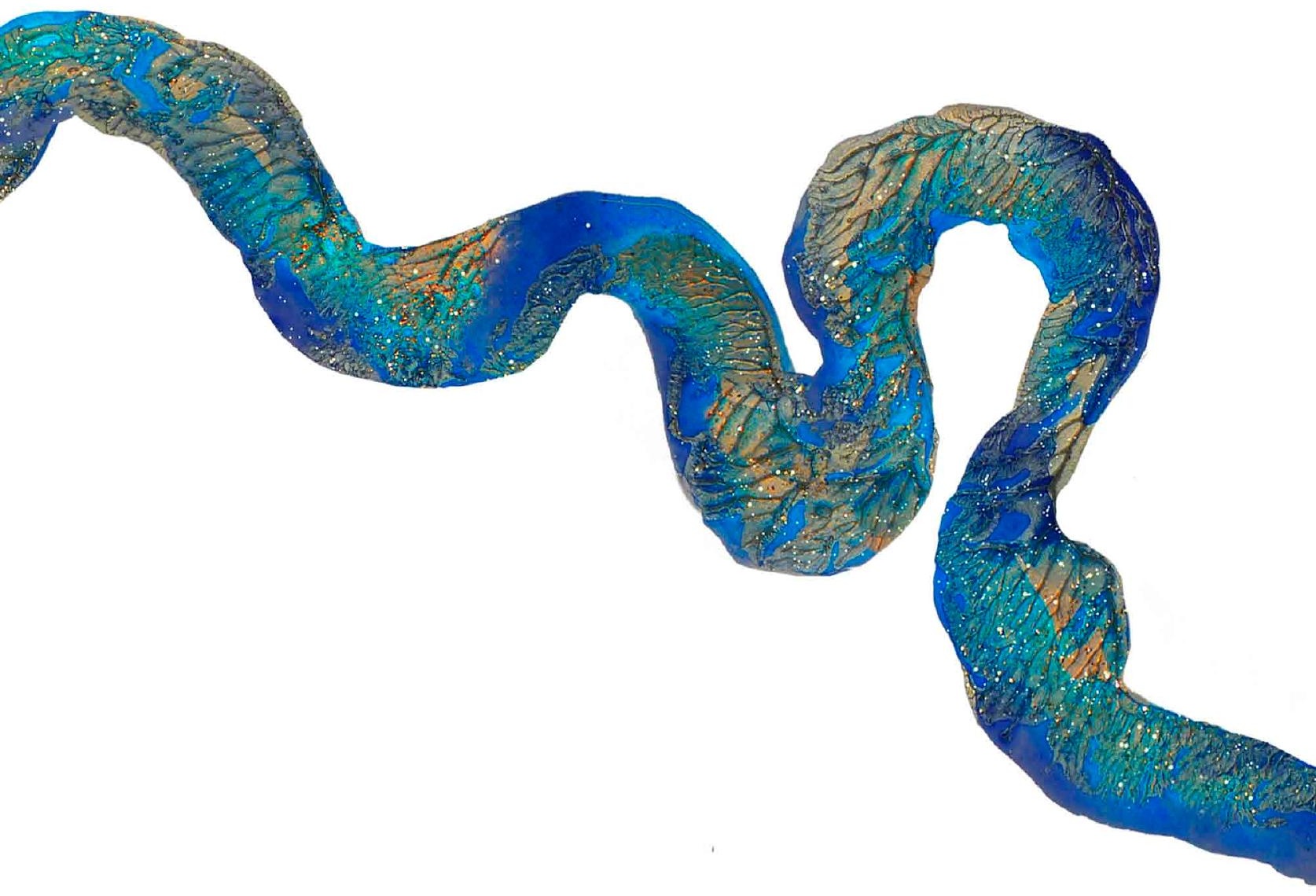




\section{GENERAL INTRODUCTION}

The microbiome consists of trillions of microbial cells with a high inter- and intraspecies variability [1]. Nobelist and visionary Joshua Lederberg proposed the expression "microbiome, to signify the ecological community of commensal, symbiotic, and pathogenic microorganisms that literally share our body space and have been all but ignored as determinants of health and disease" [2]. Research reveals that the totality of the microbes inhabiting the intestine - the gut microbiome - has thousands of times more genes than the human genome and that the bacterial cells in the human host have been estimated being in the same order as the number of human cells [1,3]. The gut microbiome seems to have a central function in the eukaryote organism, not just as digestive system or for the synthesis of essential vitamins and amino acids, but also in the development of the immune system, the maintenance of the energy balance, bile acid and lipid metabolism, detoxification of potentially harmful chemicals, prevention of infections, angiogenesis and many others [4-12]. However, since many of these findings are based on associations, the mechanisms behind the interplay between microbes and host remain to be explored.

Rapid developments and refinement of metagenomic techniques coupled with increasing computing capacities and bioinformatic tools have enabled a microbial journey of discovery up to a molecular level. While bacterial cells represent the vast majority, they are just one part of the microbiome: it is also composed of archaeal organisms, the mycobiome, the virome and other microbes such as protists and metazoans [13]. Studies of these parts of the gut microbiome, however, are still in their infancy.

The gut microbiome has become a burgeoning field of research and is proven to play a role in scientific findings in toxicology, pharmacology, oncology, neurology and many others. As research has demonstrated, it plays an important role in health and disease of the host, and can be influenced by factors such as diet, the host condition, environmental chemical exposure and drugs [14-17]. The crosstalk between microbiome and host is often based on signal molecules or metabolites [18]. The microbiome-derived metabolites can affect the host, for example via nuclear receptor signaling, leading to changes in signaling pathways including for example energy metabolism and regulation of the immune system [19].

Although the fundamental role of the microbiome in the well-being of the host is clear, science is now dealing with a bigger challenge, i.e. understanding the mechanisms by which these processes of the tight microbial-host interaction are driven. Integrative approaches that not only assess the composition of the gut microbiome but also its functionality must be implemented to unravel the complex dynamics of these interactions [11]. There are high intra- and interspecies variabilities regarding the composition of the microbiome, however, there are also indications that the functionality in terms of gene activity and metabolic output of the microbes have a lower variability [20-22] which supports assessing functional microbial changes with metabolomics approaches. 


\section{Inter- and intraspecies variability of the gut microbiome}

The composition of the gut microbiome has a high inter- and intraspecies variability in general which makes it difficult to define a healthy gut microbiome regarding the species inhabiting the gut $[1,23]$. Bacteria are the major part of microorganisms present in the gut microbiome: they outnumber eukaryotes and archaea by 2-3 orders of magnitude [1]. The gut microbiota of each individual is similar at the highest taxonomic level, the phylum: Bacteroidetes and Firmicutes are the most prominent, followed by Proteobacteria, Verrumicrobia, Actinobacteria, Fusobacteria and Cyanobacteria [24,25]. However, it shows differences at the genus and species levels because the composition of the gut microbiome is influenced by diet, genetics, lifestyle and exposure to xenobiotics. The human intestinal microbiome consists of 70 bacterial phyla that include 500 to 1,000 resident species, 7,000 to 40,000 bacterial strains, representing 1800 genera $[11,17,26]$.

In experimental studies, rodents are commonly used as animal models exploring the interaction between gastrointestinal microorganisms and the host, and to correlate changes of the gut microbiome with diseases. A comparison of the human and mouse intestinal microbiota showed considerable similarity at the genus level but large quantitative differences. Analyses of the rat intestinal microbiota revealed a bacterial diversity exceeding that of the human gut by a factor of two to three [27]. In a recent study, the core microbiota of the Sprague-Dawley rat digestive tract was assessed: at the phylum level, 21 different taxonomic groups were identified: $80 \%$ belonging to the Firmicutes, and the remaining $20 \%$ consisted of Bacteroidetes, Proteobacteria, unclassified bacteria, Tenericutes, Actinobacteria, Cyanobacteria, Deferribacteres and Deinococcus-Thermus [28]. However, only Firmicutes, Bacteroidetes, Proteobacteria and Actinobacteria were found in all samples [28]. In general, rats, the golden standard model in toxicological studies, are proposed to be more representative of the human gut microbiota than mice, and the gut bacterial communities of humanized rats more closely reflect the gut microbiota of human donors $[28,29]$. However, while many species inhabiting the human gut are well described [30,31], the majority of rodent gut bacteria remain to be cultured and characterized [20].

\section{Metabolic capacity of the gut microbiome}

The gut microbiome is known to have an immense metabolic capacity: on the one hand the bacterial cells are able to produce a wide variety of metabolites (e.g. short chain fatty acids, amino acids, vitamins and hormones), and on the other hand it can modify or further metabolize (foodborne) chemicals or pharmaceuticals. [32]. Interestingly, contrary to differences in the human and murine intestinal genera in mice almost $80 \%$ of the functional genes were identical to the human microbiome indicating a functional overlap [33]. Also in humans the variation in expression of bacterial metabolic enzymes is less diverse than the taxonomic profile [34].

The microbiome-derived metabolites can be essential for the host's health, and enable the host to harvest otherwise inaccessible nutrients [35]. With rapidly developing technologies, 
scientists are now able to unravel the microbiome-host interactions, including the identification of a number of small molecules, produced by the microbiome, which may play a role in this interaction and the host's health $[18,36]$. Knowing which molecules are produced by the microbiome and how to detect these in the host are essential points for a better understanding of the mammalian-microbiome co-metabolism of endogenous metabolites.

A prominent example of the metabolic interaction between the gut microbiome and the host are the components of the bile [37]. Bile acids are the major functional components of the bile and act as signaling molecules in the host. Via complex molecular mechanisms they can influence the gut-liver axis, the immune system and other body systems. Primary bile acids are synthesized in the liver from cholesterol and are conjugated with either taurine or glycine, and thereby gaining their amphiphilic character, before they are secreted into the bile and finally into the small intestine to emulsify lipids into micelles and assist in the nutrient absorption. The majority of bile acids is actively reabsorbed in the ileum. A minor amount escapes the reabsorption, reaches the colon and is partly excreted. When reaching the intestine primary bile acids are further metabolized by unique microbial enzymes to unconjugated, secondary and tertiary bile acids. The microorganisms possess enzymes to modify these bile acids, for example the bile salt hydrolase (BSH) that cleaves the peptide linkage between the amino acid and the primary bile acid. Furthermore, some microbes, mainly anaerobes, possess a $7 \alpha$ - dehydroxylation activity to convert primary into secondary bile acids [38]. Modified bile acids can enter the enterohepatic circulation, which means they are absorbed into the system, recycled and re-conjugated in the liver, and then resecreted into the bile and to the intestine.

Bile acids have direct or indirect antimicrobial effects, i.e. bile acids can modulate the composition of the microbiota, which in turn has an effect on the liver and consequently on the bile acid pool [39]. Yet, a change of the gut microbial community, e.g. via xenobiotics, can also lead to change of the bile acid pool which may also have implications for the host's health [40]. In general, bile acids are known to have tumor-promoting properties and are associated for example with gastrointestinal carcinogenesis and liver cancer $[41,42]$.

The gut microbiota - as the host's second liver - can also directly and indirectly affect drug and xenobiotic metabolism of the host but can also be itself affected by these compounds as recently described by Maier et al. reporting an impact of non-antibiotic drugs on human gut commensals [43]. Microbes are able to transform molecules via direct reactions like reduction, hydrolysis, removal of succinate groups, dehydroxylation, de-/acetylation, cleavage of N-oxide bonds, proteolyse, or denitration [44]. This can lead to an activation or inactivation of the drug, or the formation toxic byproducts and metabolites possessing antimicrobial activity.

It is essential to identify both the molecules produced by gut microbiota and their effects on the host, as well as understanding how xenobiotic compounds are biotransformed by resident microbiota. There is also a need to determine the effects of xenobiotic exposures 
on gut microbiota function, composition, community organization, and resulting hostmicrobiota interactions. Determining the functional endpoints rather than the composition can contribute to a better understanding of how microbial communities function, their metabolic capacities and complex interactions, also with the host.

\section{The role of the gut microbiome in toxicology and pharmacology}

The gut microbiome is increasingly recognized as a critical component in development, health and disease [45] as it has been shown to be associated with a number of diseases [46]. A shift in the microbial community, or dysbiosis, has been correlated for example to cancer, cardiovascular, immune and liver diseases, decreased mental abilities and behavioral effects $[12,15,47-50]$. Many of these correlations just hint a relationship since the mechanisms behind it are vaguely understood. However, these mechanisms or the mode of action may play an important role in the field of pharmacology and toxicology.

Considering the metabolic capacity of the gut microbiome, it has for one the potential to metabolize or modify (foodborne) chemicals or pharmaceuticals, and for another it is able to affect the host metabolism by signaling molecules or metabolites.

The consequence of the microbial metabolism for the host was demonstrated in a case in Japan where 18 patients died caused by interactions of sorivudine, an antiviral drug, with the metabolism of the cancer drug 5-fluorouracil [51]. Here, the metabolite of sorivudine produced by the gut microbiota inhibited an enzyme responsible for detoxification of the cancer drug. There are many other studies where the metabolic capacity of the microbiome was shown to affect human (drug) metabolism, for instance acetaminophen [52] or deleobuvir [53].

Further, metabolites exclusively produced by the gut microbiome can act as agonists or antagonists of nuclear receptors and hence can directly influence the host's metabolism. For instance, secondary bile acids are recognized as important signaling molecules serving as ligands for numerous nuclear receptors [54-56]. Through binding to these receptors, similar to hormones, bile acids can regulate genes involved in lipid and glucose metabolism, and energy homeostasis of the host [57,58]. It is also conceivable that an altered bile acid pool due to changes of the gut microbiome may affect the absorption or excretion of metabolites or xenobiotics. Further, it may activate or inactivate nuclear receptors in the liver or intestine, which could lead to altered plasma concentrations or a detoxification or toxification of a compound of interest.

The metabolic capacity of the gut microbiome may influence the absorption, distribution, metabolism and excretion (ADME) of a compound. However, although the microbiome can act as a first pass metabolism, it is not taken into account in the general concepts of toxicology and pharmacology. Indirect interactions have also been described, including microbial effects on the host gene expression in the liver, and activity of components of host xenobiotic metabolism, which finally can lead to an altered host (drug) metabolism $[59,60]$. 
In summary, either a change of the gut microbiome through xenobiotics, its metabolic capacity of metabolites, or the first pass metabolism of xenobiotics can influence the host's metabolism or the toxicity of a compound, which may have implications for the host's health.

However, since mechanistic studies are mostly performed in rodents, the results are difficult to extrapolate to humans and other species due to the interspecies differences in the composition of the microbiome. Furthermore, the microbiome is influenced by several factors such as a change in diet, the host condition, radiation, xenobiotics and genetics, which results in a high variability regarding the composition of the microbial community within species, but also between different species [14-16,61-63]. Hence, an essential first step is to identify host species-specific effects, e.g. to measure the endogenous metabolite profile, to identify key metabolites and to correlate them to bacterial species.

New technologies such as the omics technologies enable a deep research of the complex interaction between microbiome and host, potentially having the power to elucidate its outstanding role in the developing organism. To understand its role in toxicology and pharmacology, it is essential to determine the microbiota's function, composition, community organization, and resulting host-microbiota interactions. Omics technologies, especially metabolomics, can be suitable tools to identify microbiome-derived or associated metabolites and to reflect changes in the composition of the microbiota that could potentially lead to disease [18]. Analyzing the production of microbiota-derived metabolites is the first stage of a series of investigations that have the final goal of assessing the impact of the microbiome on the host.

\section{Metabolomics and the MetaMap ${ }^{\circledR}$ Tox database}

Small endogenous molecules within a biological sample - in its entirety called the metabolome - such as carbohydrates, amino acids, nucleic acids or fatty acids and their derivatives resulting from biochemical pathways, are defined as metabolites within the context of the omics technology metabolomics [64]. Compared to other omics technologies, metabolomics is from a statistical perspective more powerful in detecting robust effects [65] and the use of sensitive LC-MS and GC-MS techniques offers the possibility to detect a broad range of metabolites. Furthermore, metabolomics provides the opportunity of finding relevant biomarkers or patterns of change [66]. In the past years it has been shown that metabolomics can serve as a powerful tool in risk assessment, e.g. as a read-across tool, and in toxicological research $[67,68]$. Practical advantages of metabolomics include the use of, for example, urine or blood as a matrix. Thus, samples can be obtained by a less invasive method and in animal experiments it is not necessary to sacrifice the experimental animals, which also enables the rarely performed but valuable longitudinal study design. Since with metabolomics a broad range of metabolites can be quantitatively or semiquantitatively measured, metabolic fluxes can be analyzed [69]. The data obtained can be 
further used in a systems biology approach, a rapidly growing field of research, integrating these high-throughput data in computational network models [70].

In toxicological research, metabolomics can be used for identifying relevant biomarkers or metabolite patterns of change which indicate an adverse effect or organ toxicity. Measuring the metabolite profile in plasma of rats, which are a widely used animal model in toxicology, and its sensitivity to well-known toxic model compounds is an essential first step in developing the use of the plasma metabolome to detect adverse health effects [71]. Initial investigations demonstrated that after substance treatment it was possible to identify different modes of action (MoAs) or adverse outcomes in rats [71]. It was noted that the chemicals or diseases that produce a specific form of toxicity, generally through a shared mode of action, produced a subset of common specific metabolite changes. Such a common set of consistently regulated metabolites can be used to establish metabolic patterns specific for certain toxicities $[72,73]$. Using this approach in toxicology as a screening tool, various metabolite patterns for different toxicological targets and MoAs (liver, kidney, thyroid, testes, blood, nervous system and endocrine system) were established in the MetaMap ${ }^{\circledR}$ Tox [74], a metabolomics database for the identification and prediction of toxicological MoAs of new test substances. The MetaMap ${ }^{\circledR}$ Tox database has been built based upon 28-day studies in rats, adapted to the OECD 407 guideline, with blood sampling and metabolic profiling of more than 200 metabolites after 7, 14 and 28 days of test substance treatment. It contains the metabolite profiles as well as the toxicity data of approximately 800 chemicals, agrochemicals and drugs.

In the last years, research has demonstrated the impact of the gut microbiome on the host, especially on the state of the host health. It was shown that gut microbiome-derived metabolites can be found in mammalian blood and arise exclusively in the presence of gut microflora, suggesting a major interplay between bacterial and mammalian metabolism [18]. It became clear that metabolites produced or modified by the gut microbiota can be absorbed across the intestinal cell wall and can be further modified by the liver, ending up circulating in the blood stream of the host (see Figure 1). Some bacterial species and their related metabolites including their main function have been already described $[75,76]$. 


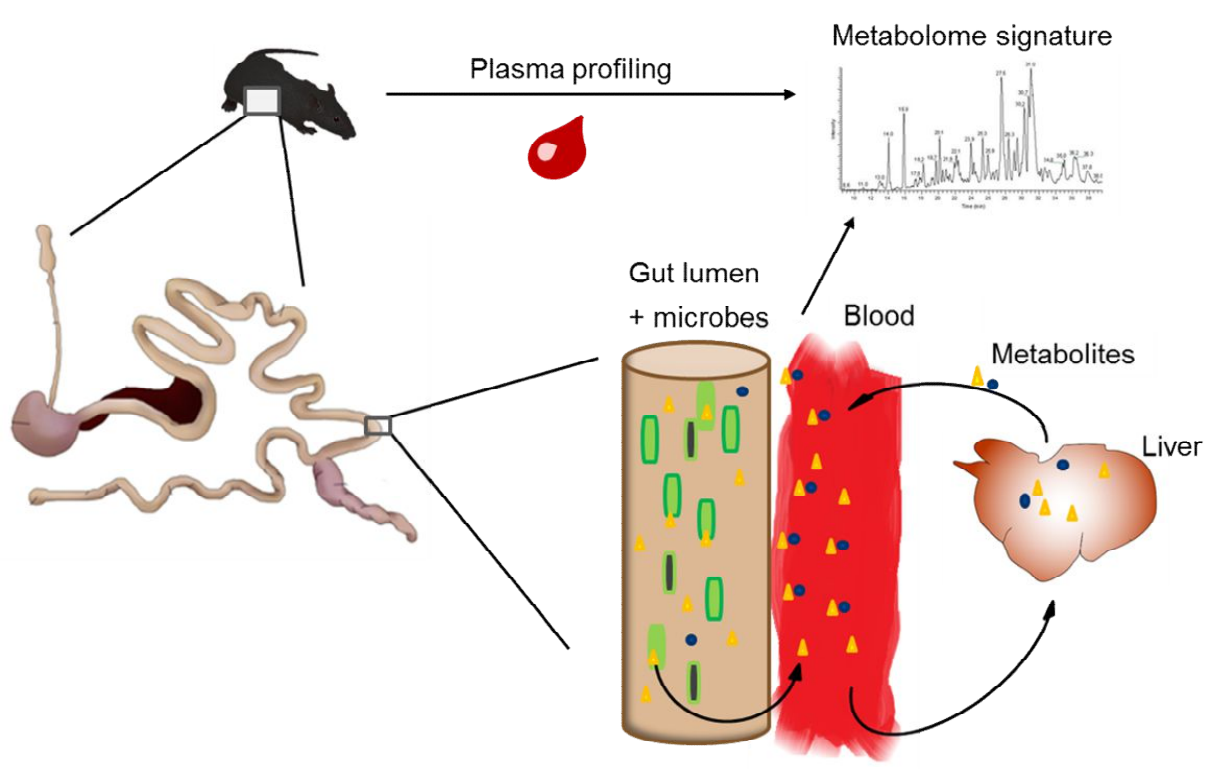

Figure 1 Influence of the gut microbiome on plasma metabolite patterns. Microbes in the lumen of the intestine produce metabolites which are absorbed into the blood. They can be further metabolized by the liver and analyzed in the plasma with metabolomics.

The crosstalk between host and microbes in the form of molecules can be measured with metabolomics which can serve here as a tool for assessing the functional status of the microbiome beyond taxonomics $[18,45]$. Especially metabolites formed by the microbiota available for uptake by the host are important to analyze since they have the potential to affect the host organism systemically.
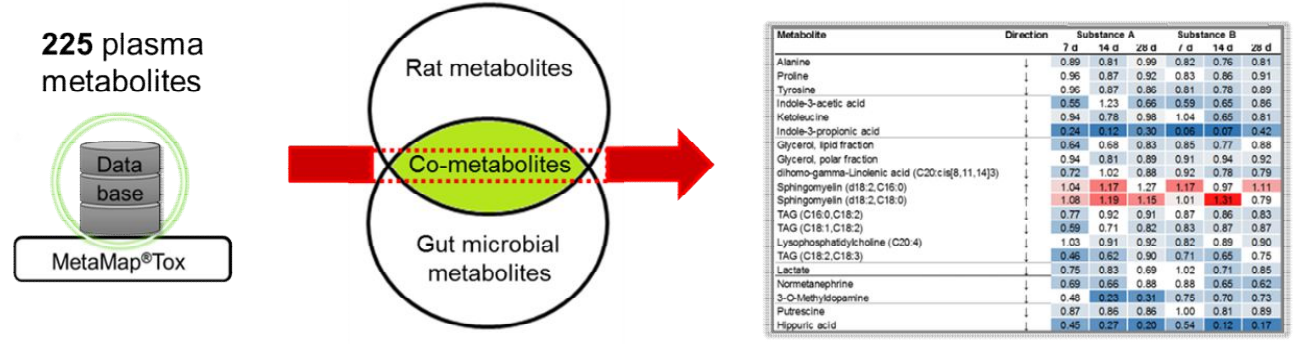

Figure 2 During a 28-day study 225 metabolites are analyzed on three time points in plasma of rats treated with test substances. The specific plasma metabolite pattern can identify and predict toxicological mode of actions of new substances. Metabolites in the blood are composed of gut microbial metabolites, endogenous metabolites of the rat, and co-metabolites of the microbial-mammalian crosstalk. 
Since the metabolite profile in plasma combines both the microbiome-derived or associated metabolites as well as the endogenous metabolites, the scope of the project was to find out which metabolites were derived specifically from the gut microbiome and what kind of impact they have on the metabolite profile (see Figure 2). Using the metabolomics approach and the existing knowledge and data availability of the MetaMap ${ }^{\circledR}$ Tox database, microbiome-related metabolites in rat plasma should be identified after treatment with substances which are known to induce a shift of the microbial community. 


\section{Aim and outline of the thesis}

The intestinal microbiota plays an important role in many host physiological aspects, also contributing to the blood metabolome that is otherwise produced by tissues of the host organism. Thus, when assessing the toxicological profile of a substance, its effect on the microbiome cannot be ignored. To enable studies on mammalian-microbiome cometabolism, understanding of effects of the microbiome's functionality on the endogenous plasma metabolome is required. The aim of this project was to obtain detailed insight in the mammalian-microbiome co-metabolism of endogenous metabolites and the extent to which the microbiome influences the plasma metabolome observed with the help of the MetaMap ${ }^{\circledR}$ Tox database and metabolic profiling.

Background information and an outline of this thesis are provided in Chapter 1, the present chapter. Also, the aims of the thesis are defined here.

Analyzing the production of microbiota-derived metabolites absorbed by the host was the first stage of the investigations, which was achieved via assessing the influence of new compounds with antibiotic activity on the host plasma metabolome as described in Chapter

2. Changes of the metabolome in plasma samples of male Wistar rats were analyzed after administration of antibiotics known to induce an artificial shift of the intestinal microbiota. This study was a first metabolic approach to establish a specific metabolome pattern for selected classes of antibiotics in the MetaMap ${ }^{\circledR}$ Tox database, and to identify plasma metabolites related to changes of the gut microbiome's functionality due to antibiotic treatment.

After successful identification of first plasma metabolites derived from the gut microbiome, further studies with six antibiotics of five different classes as well as vehicle controls were conducted. Besides plasma, also gut tissue and gut content samples were taken for further investigations.

Chapter 3 presents a study where metabolite profiling of different matrices of the gut of rats was performed, after antibiotic treatment in order to evaluate metabolite changes observed at different dose levels and in different sexes, and to identify the best tissue matrix for further investigations. The aim of this study was to assess if the same metabolites previously detected in plasma could be found in the different gut matrices, to investigate if a treatment-related effect can be observed in the analyzed matrices tissues, and if different activity spectra of antibiotics are reflected in the metabolite profiles. Furthermore, it was evaluated if metabolite changes can be observed at different dose levels and if they were sex-dependent. Lastly, the evaluations should reveal the best sample matrix for the assessment of effects of new compounds affecting the gut microbiome.

In Chapters 2 and 3, metabolite profiling was performed in different matrices. In the study described in Chapter 4, metabolite profiling in both plasma and feces of rats after lincosamides treatment was performed in order to establish specific metabolite patterns in 
the MetaMap ${ }^{\circledR}$ Tox database and to identify further key metabolites indicating gut microbial changes. Additionally, a community analysis was performed to characterize changes in the gut microbiome following treatment, vehicle treatment and general differences between the male and female animals. These evaluations help gaining a more comprehensive understanding of the effects and dynamics of microbiome alterations.

In the previous studies, various key metabolites derived from the microbiome or indicating changes of the microbiome could be identified in plasma. Since the primary bile acids could be identified as key metabolites, an additional method for a bile acid screening was set up to further elucidate changes in the bile acid metabolism. This study is described in Chapter 5. The evaluation of changes in the plasma and feces bile acid pool could help to gain a more comprehensive understanding of how microbial changes may influence the bile acid metabolism which, regarding the important role of these signaling molecules, can have far reaching consequences for the host.

Chapter 6 discusses the results of the previous chapters. Further, it provides future perspectives, discusses the limitations of the rodent model used in the studies as well as alternatives to animal studies. The final chapter, Chapter 7, provides a concluding summary of the results delivered in this thesis. 


\section{REFERENCES}

1. Sender R, Fuchs S, Milo R. Revised Estimates for the Number of Human and Bacteria Cells in the Body. PLoS Biol. 2016;14: 1-15. doi:10.1371/journal.pbio.1002533

2. Lederberg J, McCray AT. "Ome Sweet" Omics - A Genealogical Treasury of Words. Sci. 2001;15: 8.

3. Gill SR, Pop M, Deboy RT, Eckburg PB, Turnbaugh PJ, Samuel BS, et al. Metagenomic analysis of the human distal gut microbiome. Science. 2006;312: 1355-9. doi:10.1126/science. 1124234

4. Wolf G. Gut microbiota: a factor in energy regulation. Nutr Rev. 2006;64: 47-50. doi:10.1301/nr.2006.jan.47

5. Neis EPJG, Dejong CHC, Rensen SS. The Role of Microbial Amino Acid Metabolism in Host Metabolism. Nutrients. 2015;7: 2930-2946. doi:10.3390/nu7042930

6. Forbes JD, Van Domselaar G, Bernstein CN. The gut microbiota in immunemediated inflammatory diseases. Front Microbiol. 2016;7: 1-18. doi:10.3389/fmicb.2016.01081

7. Cani PD, Delzenne NM. The role of the gut microbiota in energy metabolism and metabolic disease. Curr Pharm Des. 2009;15: 1546-1558. doi:10.2174/138161209788168164

8. Sagar NM, Cree IA, Covington JA, Arasaradnam RP. The interplay of the gut microbiome, bile acids, and volatile organic compounds. Gastroenterol Res Pract. 2015;2015: 398585. doi:http://dx.doi.org/10.1155/2015/398585

9. Wilson ID, Nicholson JK. Gut microbiome interactions with drug metabolism, efficacy, and toxicity. Transl Res. Elsevier Inc.; 2017;179: 204-222. doi:10.1016/j.trsl.2016.08.002

10. Lin M, Xie Z, Zhou Y, Li Y, Ren J, Peng X, et al. Dynamic metabonomic and microbiological response of rats to lincomycin exposure: an integrated microbiology and metabonomics analysis. RSC Adv. Royal Society of Chemistry; 2015;5: 65415-65426. doi:10.1039/C5RA10626E

11. Bäumler AJ, Sperandio V. Interactions between the microbiota and pathogenic bacteria in the gut. Nature. 2016;535: 85-93. doi:10.1038/nature18849

12. Lecomte V, Kaakoush NO, Maloney C a., Raipuria M, Huinao KD, Mitchell HM, et al. Changes in Gut Microbiota in Rats Fed a High Fat Diet Correlate with Obesity-Associated Metabolic Parameters. PLoS One. 2015;10: e0126931. doi:10.1371/journal.pone.0126931

13. Virgin HW. The virome in mammalian physiology and disease. 2014;157: 142150. doi:10.1016/j.cell.2014.02.032.The

14. Modi SR, Collins JJ, Relman D a. Antibiotics and the gut microbiota. J Clin Invest. 2014;124: 4212-4218. doi:10.1172/JCI72333.themselves

15. Zhang Y-J, Li S, Gan R-Y, Zhou T, Xu D-P, Li H-B. Impacts of Gut Bacteria on Human Health and Diseases. Int J Mol Sci. 2015;16: 7493-7519. 
doi:10.3390/ijms16047493

16. David L a, Maurice CF, Carmody RN, Gootenberg DB, Button JE, Wolfe BE, et al. Diet rapidly and reproducibly alters the human gut microbiome. Nature. Nature Publishing Group; 2014;505: 559-63. doi:10.1038/nature12820

17. Rosenfeld CS. Gut Dysbiosis in Animals Due to Environmental Chemical Exposures. Front Cell Infect Microbiol. 2017;7. doi:10.3389/fcimb.2017.00396

18. Wikoff WR, Anfora AT, Liu J, Schultz PG, Lesley S a, Peters EC, et al. Metabolomics analysis reveals large effects of gut microflora on mammalian blood metabolites. Proc Natl Acad Sci U S A. 2009;106: 3698-3703. doi:10.1073/pnas.0812874106

19. Rooks MG, Garrett WS. Gut microbiota, metabolites and host immunity. Nat Rev Immunol. Nature Publishing Group; 2016;16: 341-52. doi:10.1038/nri.2016.42

20. Hugenholtz F, de Vos WM. Mouse models for human intestinal microbiota research: a critical evaluation. Cell Mol Life Sci. Springer International Publishing; 2018;75: 149-160. doi:10.1007/s00018-017-2693-8

21. McCoy KD, Geuking MB, Ronchi F. Gut microbiome standardization in control and experimental mice. Curr Protoc Immunol. 2017;2017: 23.1.1-23.1.13. doi:10.1002/cpim.25

22. Cho I, Blaser MJ. The human microbiome: at the interface of health and disease. Nat Rev Genet. 2012;13: 260-70. doi:10.1038/nrg3182

23. Nguyen TLA, Vieira-Silva S, Liston A, Raes J. How informative is the mouse for human gut microbiota research? Dis Model Mech. 2015;8: 1-16. doi: $10.1242 / \mathrm{dmm} .017400$

24. The Human Microbiome Project Consortium. Structure, function and diversity of the healthy human microbiome [Internet]. Nature. Nature Publishing Group; 2012. doi:10.1038/nature11234

25. Jost L, Devries P, Walla T, Greeney H, Chao A, Ricotta C. Partitioning diversity for conservation analyses. Divers Distrib. 2010;16: 65-76. doi:10.1111/j.14724642.2009.00626.x

26. Jovel J, Patterson J, Wang W, Hotte N, O'Keefe S, Mitchel T, et al. Characterization of the gut microbiome using $16 \mathrm{~S}$ or shotgun metagenomics. Front Microbiol. 2016;7: 1-17. doi:10.3389/fmicb.2016.00459

27. Manichanh C, Reeder J, Gibert P, Varela E, Llopis M, Antolin M, et al. Reshaping the gut microbiome with bacterial transplantation and antibiotic intake. Genome Res. 2010;20: 1411-1419. doi:10.1101/gr.107987.110

28. Li D, Chen H, Mao B, Yang Q, Zhao J, Gu Z, et al. Microbial Biogeography and Core Microbiota of the Rat Digestive Tract. Sci Rep. Nature Publishing Group; 2017;8:45840. doi:10.1038/srep45840

29. Wos-Oxley M, Bleich A, Oxley APA, Kahl S, Janus LM, Smoczek A, et al. Comparative evaluation of establishing a human gut microbial community within rodent models. Gut Microbes. 2012;3: 234-249. doi:10.4161/gmic.19934

30. Lagier JC, Khelaifia S, Alou MT, Ndongo S, Dione N, Hugon P, et al. Culture of 
previously uncultured members of the human gut microbiota by culturomics. Nat Microbiol. 2016;1. doi:10.1038/nmicrobiol.2016.203

31. Rajilić-Stojanović M, de Vos WM. The first 1000 cultured species of the human gastrointestinal microbiota. FEMS Microbiol Rev. 2014;38: 996-1047. doi:10.1111/1574-6976.12075

32. Xiao L, Feng Q, Liang S, Sonne SB, Xia Z, Qiu X, et al. A catalog of the mouse gut metagenome. Nat Biotechnol. 2015;33: 1103-1108. doi:10.1038/nbt.3353

33. Krych L, Hansen CHF, Hansen AK, van den Berg FWJ, Nielsen DS. Quantitatively Different, yet Qualitatively Alike: A Meta-Analysis of the Mouse Core Gut Microbiome with a View towards the Human Gut Microbiome. PLoS One. 2013;8. doi:10.1371/journal.pone.0062578

34. Human Microbiome Project Consortium T, Huttenhower C, Gevers D, Knight R, Abubucker S, Badger JH, et al. Structure, function and diversity of the healthy human microbiome. Nature. Nature Publishing Group; 2012;486: 207-214. doi:10.1038/nature11234

35. Bäckhed F, Ley RE, Sonnenburg JL, Peterson DA, Gordon JI. Host-bacterial mutualism in the human intestine. Science (80- ). 2005;307: 1915-1920. doi: $10.1126 /$ science. 1104816

36. Martin FPJ, Wang Y, Yap IKS, Sprenger N, Lindon JC, Rezzi S, et al. Topographical variation in murine intestinal metabolic profiles in relation to microbiome speciation and functional ecological activity. J Proteome Res. 2009;8: 3464-3474. doi:10.1021/pr900099x

37. Long SL, Gahan CGM, Joyce SA. Interactions between gut bacteria and bile in health and disease. Mol Aspects Med. Elsevier Ltd; 2017;56: 54-65. doi:10.1016/j.mam.2017.06.002

38. Ridlon JM, Kang D-J, Hylemon PB. Bile salt biotransformations by human intestinal bacteria. J Lipid Res. 2006;47: 241-259. doi:10.1194/jlr.R500013JLR200

39. Jia W, Xie G, Jia W. Bile acid-microbiota cross-talk in gastrointestinal inflammation and carcinogenesis. Nat Rev Gastroenterol Hepatol. 2018;15: 111128. doi:10.1016/j.jhsa.2009.09.008.Validity

40. Zhang Y, Limaye PB, Renaud HJ, Klaassen CD. Effect of various antibiotics on modulation of intestinal microbiota and bile acid profile in mice. Toxicol Appl Pharmacol. Elsevier Inc.; 2014;277: 138-145. doi:10.1016/j.taap.2014.03.009

41. Yoshimoto S, Loo TM, Atarashi K, Kanda H, Sato S, Oyadomari S, et al. Obesityinduced gut microbial metabolite promotes liver cancer through senescence secretome. Nature. 2013;499: 97-101. doi:10.1038/nature12347

42. Fu T, Zhao X, Evans RM. Liver Cancer Checks in When Bile Acid Clocks Out. Cancer Cell. Elsevier Inc.; 2016;30: 827-828. doi:10.1016/j.ccell.2016.11.012

43. Maier L, Pruteanu M, Kuhn M, Zeller G, Telzerow A, Anderson EE, et al. Extensive impact of non-antibiotic drugs on human gut commensals. Nature. Nature Publishing Group; 2018; doi:10.1038/nature25979.

44. Sousa T, Paterson R, Moore V, Carlsson A, Abrahamsson B, Basit AW. The 
gastrointestinal microbiota as a site for the biotransformation of drugs. Int $\mathrm{J}$ Pharm. 2008;363: 1-25. doi:10.1016/j.ijpharm.2008.07.009

45. Silbergeld EK. The Microbiome. Toxicol Pathol. 2017;45: 190-194. doi:10.1177/0192623316672073

46. Schroeder BO, Bäckhed F. Signals from the gut microbiota to distant organs in physiology and disease. Nat Med. 2016;22: 1079-1089. doi:10.1038/nm.4185

47. Velasquez-Manoff M. 2. Gut Microbiome: The Peacekeepers. Nature. 2015;518: S3-S11. doi:10.1038/518S3a

48. Guarner F, Malagelada JR. Gut flora in health and disease. Lancet. 2003;361: 1831. doi:10.1016/S0140-6736(03)12489-0

49. Kirpich IA, Marsano LS, McClain CJ. Gut-liver axis, nutrition, and non-alcoholic fatty liver disease. Clinical Biochemistry. 2015. pp. 923-930. doi:10.1016/j.clinbiochem.2015.06.023

50. Toh MC, Allen-Vercoe E. The human gut microbiota with reference to autism spectrum disorder: considering the whole as more than a sum of its parts. Microb Ecol Health Dis. 2015;26: 26309. doi:10.3402/mehd.v26.26309

51. Okuda H, Ogura K, Kato A, Takubo H, Watabe T. A possible mechanism of eighteen patient deaths caused by interactions of sorivudine, a new antiviral drug, with oral 5-fluorouracil prodrugs. J Pharmacol Exp Ther. 1998;287: 791-799.

52. Clayton TA, Baker D, Lindon JC, Everett JR, Nicholson JK. Pharmacometabonomic identification of a significant host-microbiome metabolic interaction affecting human drug metabolism. Proc Natl Acad Sci U S A. 2009;106: 14728-14733. doi:10.1073/pnas.0904489106

53. McCabe M, Sane RS, Keith-Luzzi M, Xu J, King I, Whitcher-Johnstone a., et al. Defining the Role of Gut Bacteria in the Metabolism of Deleobuvir: In Vitro and In Vivo Studies. Drug Metab Dispos. 2015;43: 1612-1618. doi:10.1124/dmd.115.064477

54. Makishima M, Lu TT, Xie W, Whitfield GK, Domoto H, Evans RM, et al. Vitamin $\mathrm{D}$ receptor as an intestinal bile acid sensor. Science (80- ). 2002;296: 1313-1316. doi:10.1126/science. 1070477

55. Maruyama T, Miyamoto Y, Nakamura T, Tamai Y, Okada H, Sugiyama E, et al. Identification of membrane-type receptor for bile acids (M-BAR). Biochem Biophys Res Commun. 2002;298: 714-719. doi:10.1016/S0006-291X(02)02550-0

56. Xie W, Radominska-Pandya A, Shi Y, Simon CM, Nelson MC, Ong ES, et al. An essential role for nuclear receptors SXR/PXR in detoxification of cholestatic bile acids. Proc Natl Acad Sci. 2001;98: 3375-3380. doi:10.1073/pnas.051014398

57. Hylemon PB, Zhou H, Pandak WM, Ren S, Gil G, Dent P. Bile acids as regulatory molecules. J Lipid Res. 2009;50: 1509-1520. doi:10.1194/jlr.R900007-JLR200

58. Vítek L, Haluzík M. The role of bile acids in metabolic regulation. J Endocrinol. 2016;228: R85-R96. doi:10.1530/JOE-15-0469

59. Haiser HJ, Turnbaugh PJ. Is it time for a metagenomic basis of therapeutics? Science. 2012;336: 1253-5. doi:10.1126/science.1224396 
60. Claus SP, Ellero SL, Berger B, Krause L, Bruttin A, Molina J, et al. ColonizationInduced Host-Gut Microbial Metabolic Interaction. MBio. 2011;2: e00271-10. doi:10.1128/mBio.00271-10.Editor

61. Huse SM, Dethlefsen L, Huber J a., Welch DM, Relman D a., Sogin ML. Exploring Microbial Diversity and Taxonomy Using SSU rRNA Hypervariable Tag Sequencing. PLoS Genet. 2008;4: e1000255. doi:10.1371/journal.pgen.1000255

62. Arumugam M, Raes J, Pelletier E, Paslier D Le, Yamada T, Mende DR, et al. Enterotypes of the human gut microbiome. 2011; 1-7. doi:10.1038/nature09944

63. Ottman N, Smidt H, de Vos WM, Belzer C. The function of our microbiota: who is out there and what do they do? Front Cell Infect Microbiol. 2012;2: 1-11. doi:10.3389/fcimb.2012.00104

64. Lindon JC, Holmes E, Bollard ME, Stanley EG, Nicholson JK. Metabonomics technologies and their applications in physiological monitoring, drug safety assessment and disease diagnosis. Biomarkers. 2004;9: 1-31. doi:10.1080/13547500410001668379

65. van Ravenzwaay B, Herold M, Kamp H, Kapp MD, Fabian E, Looser R, et al. Metabolomics: A tool for early detection of toxicological effects and an opportunity for biology based grouping of chemicals-From QSAR to QBAR. Mutat Res - Genet Toxicol Environ Mutagen. Elsevier B.V.; 2012;746: 144-150. doi:10.1016/j.mrgentox.2012.01.006

66. Looser R, Krotzky AJ, Trethewey N. Metabolite profiling with GC-MS and LC-MS - a key tool for contemporary biology. In: Vaidyanathan S, Harrigan G, Goodacre R, editors. Metabolome analyses - Strategies for systems biology. New York: Springer; 2005. pp. 103-118.

67. Beger RD, Sun J, Schnackenberg LK. Metabolomics approaches for discovering biomarkers of drug-induced hepatotoxicity and nephrotoxicity. Toxicology and applied pharmacology. 2010. pp. 154-166. doi:10.1016/j.taap.2009.11.019

68. van Ravenzwaay B, Sperber S, Lemke O, Fabian E, Faulhammer F, Kamp H, et al. Metabolomics as read-across tool: A case study with phenoxy herbicides. Regul Toxicol Pharmacol. Elsevier Ltd; 2016;81: 288-304. doi:10.1016/j.yrtph.2016.09.013

69. Fernie AR, Trethewey RN, Krotzky AJ, Willmitzer L. Metabolite profiling: from diagnostics to systems biology. Nat Rev Mol Cell Biol. 2004;5: 763-9. doi:10.1038/nrm1451

70. Heinken A, Thiele I. Systems biology of host-microbe metabolomics. WIREs Syst Biol Med. 2015;7: 195-219. doi:10.1002/wsbm.1301

71. van Ravenzwaay B, Cunha GCP, Leibold E, Looser R, Mellert W, Prokoudine a., et al. The use of metabolomics for the discovery of new biomarkers of effect. Toxicol Lett. 2007;172: 21-28. doi:10.1016/j.toxlet.2007.05.021

72. Mattes W, Davis K, Fabian E, Greenhaw J, Herold M, Looser R, et al. Detection of hepatotoxicity potential with metabolite profiling (metabolomics) of rat plasma. Toxicol Lett. Elsevier Ireland Ltd; 2014;230: 467-478. doi:10.1016/j.toxlet.2014.07.021 
73. Kamp H, Strauss V, Wiemer J, Leibold E, Walk T, Mellert W, et al. Reproducibility and robustness of metabolome analysis in rat plasma of 28-day repeated dose toxicity studies. Toxicol Lett. 2012;215: 143-149.

74. Behr C, Kamp H, Fabian E, Krennrich G, Mellert W, Peter E, et al. Gut microbiome-related metabolic changes in plasma of antibiotic-treated rats. Arch Toxicol. Springer Berlin Heidelberg; 2017;91: 3439-3454. doi:10.1007/s00204017-1949-2

75. Heinken A, Thiele I. Systematic prediction of health - Relevant humanmicrobial co-metabolism through a computational framework. Gut Microbes. 2015;6: 85-92. doi:10.1080/19490976.2015.1023494

76. Nicholson JK, Holmes E, Kinross J, Burcelin R, Gibson G, Jia W, et al. Host-Gut Microbiota Metabolic Interactions. 2012;108: 1262-1268. 

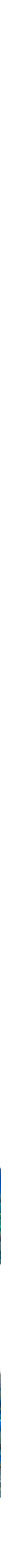


\section{Chapter 2}

\section{Gut microbiome-related metabolic changes in plasma of antibiotic-treated rats}

C. Behr, H. Kamp, E. Fabian, G. Krennrich, W. Mellert, E. Peter, V. Strauss, T. Walk, I.M.C.M. Rietjens, B. van Ravenzwaay

Published in: Archives of Toxicology (2017) 91:3439-3454 


\section{AbSTRaCt}

The intestinal microbiota contributes to the metabolism of its host. Adequate identification of the microbiota's impact on the host plasma metabolites is lacking. As antibiotics have a profound effect on the microbial composition and hence on the mammalian-microbiota cometabolism, we studied effects of antibiotics on the "functionality of the microbiome" defined as the production of metabolites absorbed by the host.

This metabolomics study presents insights into the mammalian-microbiome co-metabolism of endogenous metabolites. To identify plasma metabolites related to microbiome changes due to antibiotic treatment, we have applied broad-spectrum antibiotics belonging to the class of aminoglycosides (neomycin, gentamicin), fluoroquinolones (moxifloxacin, levofloxacin) and tetracyclines (doxycycline, tetracycline). These were administered orally for 28 days to male rats including blood sampling for metabolic profiling after 7, 14 and 28 days. Fluoroquinolones and tetracyclines can be absorbed from the gut, whereas aminoglycosides are poorly absorbed. Hippuric acid, indole-3-acetic acid and glycerol were identified as key metabolites affected by antibiotic treatment, beside changes mainly concerning amino acids and carbohydrates. Inter alia, effects on indole-3-propionic acid were found to be unique for aminoglycosides, and on 3-indoxylsulfate for tetracyclines. For each class of antibiotics specific metabolome patterns could be established in the MetaMap ${ }^{\circledR}$ Tox data base, which contains metabolome data for more than 550 reference compounds. The results suggest that plasma based metabolic profiling (metabolomics) could be a suitable tool to investigate the effect of antibiotics on the functionality of the microbiome and to obtain insight into the mammalian-microbiome co-metabolism. 


$\begin{array}{ll}\text { ABBREVIATIONS } \\ \text { CKD } & \text { chronic kidney disease } \\ \text { HA } & \text { hippuric acid } \\ \text { IAA } & \text { indole-3-acetic acid } \\ \text { IPA } & \text { indole-3-propionic acid } \\ \text { IS } & \text { 3-indoxylsulfate } \\ \text { GC } & \text { gas chromatography } \\ \text { LC } & \text { liquid chromatography } \\ \text { MOA } & \text { mode of action } \\ \text { MS } & \text { mass spectrometry } \\ \text { SPE } & \text { solid phase extraction }\end{array}$

\section{INTRODUCTION}

The intestinal microbiota is known to produce a variety of metabolites (e.g. short chain fatty acids, amino acids, vitamins and hormones) which are essential for the host's health. However, it has also been shown to be associated with a number of diseases (Cho and Blaser 2012; Zhang et al. 2015). A shift in the microbial community, or dysbiosis, has been correlated with diseases such as cancer, cardiovascular, immune and liver diseases, decreased mental abilities and behavioral effects (Guarner and Malagelada 2003; Kirpich et al. 2015; Lecomte et al. 2015; Toh and Allen-Vercoe 2015; Velasquez-Manoff 2015; Zhang et al. 2015). Evidence suggests a strong interaction between the gut microbiome, the gut and the host's wellbeing. We are now starting to unravel the microbiome-host interactions, including a number of small molecules, produced by the microbiome, which may play a role in this interaction (Martin et al. 2009; Wikoff et al. 2009). Knowing which molecules are produced by the microbiome, their role in the host's well-being and how to detect these in the host are essential points for a better understanding of the mammalian-microbiome cometabolism of endogenous metabolites.

The human microbiome consists of 100 trillion cells of microbes with a high inter- and intraspecies variability (Huse et al. 2008; Arumugam et al. 2011; Ottman et al. 2012). Several factors such as a change in diet, the host condition, radiation, and toxicological insult (e.g. antibiotics) can influence the microbiome (David et al. 2014; Modi et al. 2014; Zhang et al. 2015). A concept to assess risks associated with microbiome changes is the investigation of the microbiome's functionality (defined as the production of metabolites absorbed by the host). Therefore, it is essential to determine the microbiota'scomposition, organization and the host-microbiome interactions. Omics technologies, especially metabolomics, could be suitable tools to identify microbiome derived metabolites and to reflect changes in the composition of the microbiota that could potentially lead to disease (Wikoff et al. 2009). 
In the past years it has been shown that metabolomics can serve as a powerful tool in risk assessment and toxicological research (Beger et al. 2010). Within the context of metabolomics, metabolites are defined as small endogenous compounds such as carbohydrates, amino acids, nucleic acids or fatty acids and their derivatives resulting from biochemical pathways (Lindon et al. 2004). Practical advantages of metabolomics include the use of, for example, blood as a matrix. Samples can be obtained by a less invasive method and it is not necessary to sacrifice animals, also making time course analysis possible. The use of sensitive LC-MS and GC-MS techniques offers the possibility to detect a broad range of metabolites and thus is one increment to increase the chance of finding relevant biomarkers or patterns of change (Looser et al. 2005).

The interpretation of biomarkers or patterns of change in plasma metabolites requires insight into to what extent (adverse) effects on the host and microbiota are reflected in the plasma metabolite profile. Measuring the metabolome (metabolite profile) in rat plasma and its sensitivity to well known toxic model compounds is an essential first step in developing the use of the plasma metabolome to detect adverse health effects (van Ravenzwaay et al. 2007). Therefore, the aim of the present study was to identify the metabolome in blood samples of Wistar rats after administration of antibiotics known to modify the intestinal microbiota, which is likely to contribute essentially to the plasma metabolite profile of the mammalian host organism (Wikoff et al. 2009; Zheng et al. 2011; Nicholson et al. 2012; Marcobal et al. 2013; Heinken and Thiele 2015a; Marcobal et al. 2015). Some phyla/strains and their related main/most important metabolites including their main function have been described (Heinken and Thiele, 2015b; Nicholson et al., 2012, M Fischbach, personal communication). With the help of an artificial shift of the microbiota, e.g. with antibiotics, it is conceivable to specifically identify microbiota-related metabolites in the blood and to correlate these metabolites with their bacterial producers.

In this study, a metabolic blood profiling after oral administration of broad-spectrum antibiotics of different classes (aminoglycosides, tetracyclines, and fluoroquinolones) in male Wistar rats was performed. Relative levels of endogenous metabolites in treated rats were compared to the levels of these metabolites in untreated controls. To distinguish between metabolite changes caused by systemic toxicity of the antibiotics and microbiota related changes, we applied aminoglycosides. When orally administered, this class of antibiotics is poorly absorbed from the gut $(1-6 \%)$ (Renshaw et al.; Brown and Riviere 1991; Dhillon 2006). Thus, a change in the plasma metabolite pattern can be associated with changes in the functionality of the gut microbiota and effects on plasma metabolites due to organ toxicity are negligible. To interpret the metabolome data obtained, the data base MetaMap ${ }^{\circledR}$ Tox with more than 550 reference compounds was used. This data base is used to identify and predict the systemic toxicity or toxicological modes of action (MOA) of new compounds by creating sets of common metabolite level changes (metabolite 
pattern) (van Ravenzwaay et al. 2007; Strauss et al. 2009; van Ravenzwaay et al. 2010a; Van Ravenzwaay et al. 2012; Kamp et al. 2012b).

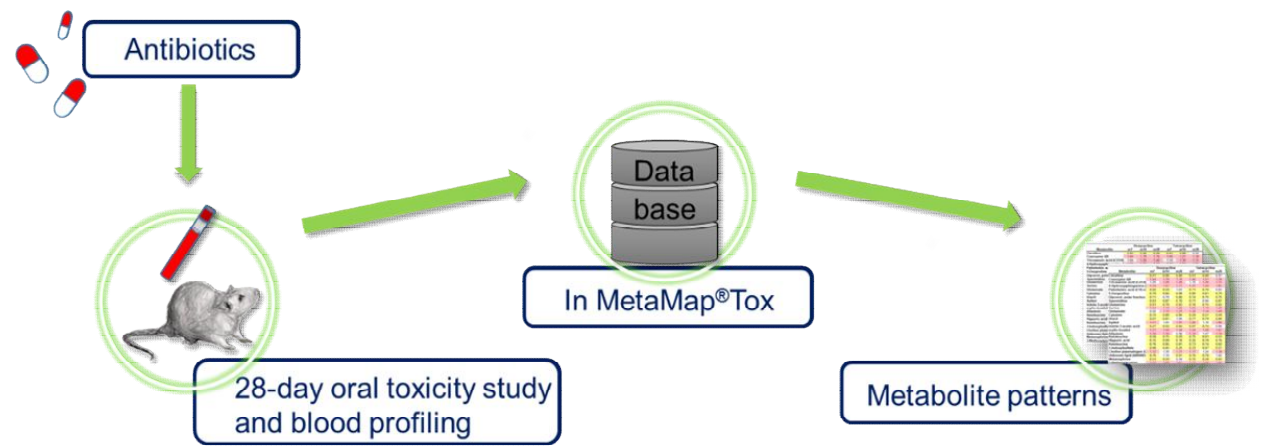

Figure 1 Metabolic profiling in different gut matrices of antibiotic treated rats. To identify plasma metabolites related to microbiome changes due to antibiotic treatment, we have employed a metabolomics approach. Broadspectrum antibiotics (Vancoymcin, Streptomycin and Roxithromycin), with each a different activity spectrum, were administered orally to rats in a toxicity study. Feces, cecum content and gut tissue (jejunum, ileum, cecum, colon, rectum) were sampled for metabolic profiling on day 28 of the study.

Analyzing the production of microbiota-derived metabolites absorbed by the host is the first stage of our investigations, which aims at assessing toxicological risks of new compounds with antibiotic activity.

This study here is a first metabolic approach to 1) establish a specific metabolome pattern for selected classes of antibiotics in the data base MetaMap ${ }^{\circledR T o x}$, and 2) identify plasma metabolites related to changes of the gut microbiome's functionality due to antibiotic treatment.

\section{Materials ANd Methods}

Five independent studies were performed according to the OECD 407 protocol. Animal handling, treatment, and clinical examinations have been described earlier (van Ravenzwaay et al. 2007; van Ravenzwaay et al. 2010b; Strauss et al. 2012; Van Ravenzwaay et al. 2012; Kamp et al. 2012b).

\section{Ethics statement}

The studies were approved by the BASF Animal Welfare Body, with the permission of the local authority, the Landesuntersuchungsamt Rheinland-Pfalz (reference number 23177 07). The laboratory is AAALAC (Association for Assessment and Accreditation of Laboratory Animal Care International) certified since 2007. 


\section{Animals and Maintenance Conditions}

Briefly, Wistar rats (CrI:WI(Han)) were supplied by Charles River, Germany, and were approx. 70 days old at the beginning of the studies. The animals were maintained in an airconditioned room at a temperature of 20 to $24^{\circ} \mathrm{C}$, a relative humidity of 30 to $70 \%$, and a 12 hour light / 12 hour dark cycle. Ground Kliba mouse/rat maintenance diet was supplied by Provimi Kliba SA, Kaiseraugst, Switzerland. The diet and drinking water were available ad libitum (except before blood sampling) and regularly assayed for chemical contaminants and the presence of microorganisms.

\section{Treatment of animals with compounds}

Treatment groups of five animals per group were compared with a dietary control group (10 male rats) irrespective of the administration route of the antibiotics (doxycycline and levofloxacin were applied in the same study, i.e. these antibiotics were compared against the same control animals). This was done in order to compare the groups with reference compounds of the MetaMap ${ }^{\circledR}$ Tox database based on uniform diet control groups. In former studies the metabolome of control groups with different administration routes had been compared: rats gavaged with $0.5 \%$ carboxymethyl cellulose (CMC; administration route of some antibiotics in the present study) had less than 5\% significantly altered metabolites compared to diet controls (Welch t-test, $\mathrm{p}<0.05$ ). This is regarded as an incidental change, which does not interfere with antibiotic-related effects of the present treatment-groups. Dose-levels (according to literature data (U.S. Food and Drug Administration; Dietz 1989; van Koten-Vermeulen and van Leeuwen 1995; Dalhoff 2001; Kuroda et al. 2001; Center for Drug Evaluation and Research 2003; Tanaka et al. 2004; Schoonen et al. 2007; U.S. Food and Drug Administration 2008; Center for Drug Evaluation and Research 2013)), routes of administration and form of preparation of the antibiotics are summarized in Table 1.

Table 1 Compounds used, dose levels, routes of administration (oral administration by gavage or administration in feed), form of preparation, and class of antibiotics.

\begin{tabular}{|c|c|c|c|c|}
\hline Treatment & Dose/day & Route & Form of preparation & $\begin{array}{l}\text { Class of } \\
\text { antibiotics }\end{array}$ \\
\hline Tetracycline Hydrochloride & approx. $5,000 \mathrm{mg} / \mathrm{kg}$ body weight & Diet & - & Tetracyclines \\
\hline Doxycycline Monohydrate & $250 \mathrm{mg} / \mathrm{kg}$ body weight & gavage & $\begin{array}{l}\text { in water containing } \\
0.5 \% \mathrm{CMC}^{\mathrm{a}}\end{array}$ & Tetracyclines \\
\hline Moxifloxacin Hydrochloride & $750 \mathrm{mg} / \mathrm{kg}$ body weight & gavage & $\begin{array}{l}\text { in water containing } \\
0.5 \% \mathrm{CMC}^{\mathrm{a}}\end{array}$ & Fluoroquinolones \\
\hline Levofloxacin & $200 \mathrm{mg} / \mathrm{kg}$ body weight & gavage & $\begin{array}{l}\text { in water containing } \\
0.5 \% \mathrm{CMC}^{\mathrm{a}}\end{array}$ & Fluoroquinolones \\
\hline Neomycin Sulfate & $250 \mathrm{mg} / \mathrm{kg}$ body weight & gavage & in water & Aminoglycosides \\
\hline Gentamicin Sulfate & $100 \mathrm{mg} / \mathrm{kg}$ body weight & gavage & in water & Aminoglycosides \\
\hline
\end{tabular}

${ }^{a}$ carboxymethyl cellulose: Tylose CB30000 


\section{Blood Sampling}

Between 7:30 and 10:30 h, blood samples were taken from the retro-orbital sinus in all rats under isoflurane anesthesia (1.0 ml K-EDTA blood on study days 7, 14 and 28) after a fasting period of $16-20$ hours and one day after the last administration of the test substances. The blood samples were centrifuged $\left(10{ }^{\circ} \mathrm{C}, 2000 \mathrm{x} \mathrm{g}, 10\right.$ minutes $)$ and the EDTA plasma was separated. The EDTA plasma samples were covered with nitrogen and frozen at $-80^{\circ} \mathrm{C}$ until metabolite profiling was performed.

\section{Clinical Examinations}

All animals were checked daily for any clinically abnormal signs and mortalities. Food consumption was determined on study days 6, 13, 20 and 27. Body weight was determined before the start of the administration period in order to randomize the animals and on study days $0,6,13,20$ and 27 . At the end of the treatment period, the animals were sacrificed by decapitation under Isoflurane anesthesia. Metabolome evaluation of the blood samples was performed for all control, and treated animals.

\section{Metabolite Profiling}

For mass spectrometry-based metabolite profiling analysis, K-EDTA plasma samples taken on study days 7, 14 and 28 were extracted by a proprietary method as described below. Three types of mass spectrometry analysis were applied to all samples: GC-MS (gas chromatography-mass spectrometry) and LC-MS/MS (liquid chromatography-MS/MS) were used for broad profiling (van Ravenzwaay et al. 2007). SPE-LC-MS/MS (Solid phase extraction-LC-MS/MS) was applied for the determination of catecholamine and steroid hormone levels. Proteins were removed from plasma samples by precipitation using acetonitrile. Subsequently polar and non-polar fractions were separated for both GC-MS and LC-MS/MS analysis by adding water and a mixture of ethanol and dichloromethane. For GC-MS analysis, the non-polar fraction was treated with methanol under acidic conditions to yield the fatty acid methyl esters derived from both free fatty acids and hydrolyzed complex lipids. The non-polar and polar fractions were further derivatized with O-methyl-hydroxylamine hydrochloride and pyridine to convert oxo-groups to O-methyloximes and subsequently with a silylating agent before analysis (Roessner et al. 2000). For LC-MS analysis, both fractions were reconstituted in appropriate solvent mixtures. HPLC was performed by gradient elution using methanol/water/formic acid on reversed phase separation columns. Mass spectrometric detection technology was applied which allows target and high sensitivity MRM (Multiple Reaction Monitoring) profiling in parallel to a full screen analysis (WO2003073464).

For GC-MS and LC-MS/MS profiling, data were normalized to the median of reference samples which were derived from a pool formed from aliquots of all samples to account for inter- and intra-instrumental variation. Steroid hormones, catecholamines and their metabolites were measured by online SPE-LC-MS/MS (Solid phase extraction-LC- 
MS/MS) (Yamada et al. 2002). The method resulted in 225 semi-quantitative analytes, 171 of which are chemically identified and 54 are structurally unknown.

\section{Statistics}

The data were analyzed by univariate and multivariate statistical methods. The sex- and day-stratified heteroscedastic t-test ("Welch test") was applied to compare metabolite levels of dose groups with respective controls. Hereafter these ratios are referred to as 'relative abundance in plasma'. The p-values, t-values, and ratios of corresponding group medians were collected as metabolic profiles and fed into the MetaMap ${ }^{\circledR}$ Toxdatabase. For a detailed description on the use of statistics in MetaMap ${ }^{\circledR}$ Tox see van Ravenzwaay et al. (Ravenzwaay et al. 2016). Whenever we write "significantly", "statistically significantly" is meant. Briefly, the best balance for finding the maximum number of truly regulated metabolites while minimizing the number of false positive regulated metabolites is obtained at a p-value of 0.15 . Therefore, for pattern recognition p-values between $0.1-0.2$ are used in the data base.

\section{Establishment of Metabolite Pattern in Metamap ${ }^{\circledR}$ Tox}

Patterns of characteristically changed metabolites for a certain toxicological mode of action were developed from the metabolite profiles as described before (Kamp et al. 2012b). In this study, characteristic metabolite changes were identified based on two antibiotics of the same class (see Table 1) from the MetaMap ${ }^{\circledR}$ Tox data base to establish a metabolite profile. After identification of the significantly changed metabolites and a consistency check through an expert panel, the pattern is validated against the data base and should not identify compounds in the MetaMap ${ }^{\circledR}$ Tox with a different toxicological mode of action.

\section{Results}

\section{Clinical signs}

There were no mortalities observed in any of the animals, which received the different antibiotics, or signs of clinical toxicity, except for moxifloxacin (slight salivation) and tetracycline (piloerection). Table 2 presents the results on food consumption and effects on body weight upon different treatments. Food consumption was reduced in the animals receiving tetracycline. With the exception of gentamicin, there was a reduction in food consumption in all test groups on day 7 , and occasionally also on day 14 . Towards the end of the study (day 28), food consumption had returned to levels close to controls for these compounds. Observed changes in body weight were in the range of $-10 \%$ of the controls, showing that the MTD (maximum tolerated dose) was not exceeded. 
Table 2 Relative changes in body weight and food consumption of male Crl:Wi(Han) rats $(\mathrm{N}=5$ per group) dosed for 4 weeks compared to the corresponding controls. Data were collected on study days 6,13 and 27 , data in in yellow boxes are statistically significantly decreased $(\mathrm{p}<0.05)$ compared to controls $(\mathrm{N}=10$ per group).

\begin{tabular}{lccc}
\hline Antibiotic & Day & $\begin{array}{l}\text { Body } \\
\text { Weight }\end{array}$ & $\begin{array}{l}\text { Food } \\
\text { Consumption }\end{array}$ \\
\hline Tetracycline & 6 & 0.91 & 0.58 \\
hydrochloride & 13 & 0.91 & 0.82 \\
& 27 & 0.89 & 0.99 \\
Doxycycline & 6 & 0.97 & 0.86 \\
monohydrate & 13 & 0.95 & 0.84 \\
& 27 & 0.94 & 1.02 \\
Gentamicin & 6 & 0.96 & 0.93 \\
sulfate & 13 & 0.95 & 0.96 \\
& 27 & 0.97 & 1.08 \\
Neomycin & 6 & 1.01 & 0.83 \\
sulfate & 13 & 0.99 & 0.99 \\
& 27 & 0.96 & 1.02 \\
Moxifloxacin & 6 & 0.95 & 0.66 \\
hydrochloride & 13 & 0.95 & 0.87 \\
& 27 & 0.88 & 0.97 \\
Levofloxacin & 6 & 0.94 & 0.90 \\
& 13 & 0.92 & 0.95 \\
& 27 & 0.90 & 1.15 \\
\hline
\end{tabular}

\section{Metabolomics}

The outcomes derived from the metabolome data analysis for the three different classes of antibiotics are presented in Figs. 2, 3 and 4. Each metabolite value of a treated group was evaluated against the value of the concurrent control and expressed as relative (foldchange) value of the control. For each class of antibiotics specific metabolome patterns were established using a p-value of 0.2 .

Key metabolites affected by antibiotic treatment of all three classes were hippuric acid (HA), indole-3-acetic acid (IAA) and glycerol, in addition antibiotic class specific changes were identified in metabolites belonging to amino acids, lipids and carbohydrates.

\section{Aminoglycosides}

After treatment with aminoglycosides 17 plasma metabolites were significantly decreased and two were significantly increased (sphingomyelin (d18:2/16:0) and (d18:2:18:0)) compared to the control group (see Fig. 2). Changes were seen in amino acids and related, complex lipids, fatty acids and related, energy metabolism and related, hormones, signal substances and related as well as miscellaneous. At all three time points (day 7, 14, 28) and for both aminoglycosides tested, indole-3-propionic acid (IPA) and hippuric acid were remarkably decreased. The lowest relative abundances in plasma for IPA were 0.12 (neomycin) and 0.06 (gentamicin), equivalent to a 12-fold and 16-fold decrease relative to control values. For both substances the relative abundance in plasma for hippuric acid were around 0.5 on day 7 and fall to 0.20 for neomycin and 0.17 for gentamicin on day 28 . As for the patterns of tetracyclines and fluoroquinolones (see Figs. 3 and 4), the relative abundance in plasma for indole-3-acetic acid was also decreased for gentamicin and 
neomycin. Low values compared to the control, $0.23-0.48$, could also be found for $3-\mathrm{O}-$ methyldopamine after neomycin treatment.

\begin{tabular}{|c|c|c|c|c|c|c|c|c|}
\hline \multirow[t]{2}{*}{ Metabolite } & \multirow[t]{2}{*}{ Direction } & \multicolumn{3}{|c|}{ Neomycin sulfate } & \multicolumn{3}{|c|}{ Gentamicin sulfate } & \multirow[t]{2}{*}{ Metabolite class } \\
\hline & & $7 \mathrm{~d}$ & $14 \mathrm{~d}$ & $28 \mathrm{~d}$ & $7 \mathrm{~d}$ & $14 \mathrm{~d}$ & $28 \mathrm{~d}$ & \\
\hline Alanine & $\downarrow$ & 0.89 & 0.81 & 0.99 & 0.82 & 0.76 & 0.81 & \multirow[t]{3}{*}{ Amino acids } \\
\hline Proline & $\downarrow$ & 0.96 & 0.87 & 0.92 & 0.83 & 0.86 & 0.91 & \\
\hline Tyrosine & $\downarrow$ & 0.96 & 0.87 & 0.86 & 0.81 & 0.78 & 0.89 & \\
\hline Indole-3-acetic acid & $\downarrow$ & 0.55 & 1.23 & 0.66 & 0.59 & 0.65 & 0.86 & \multirow[t]{3}{*}{ Amino acids related } \\
\hline Ketoleucine & $\downarrow$ & 0.94 & 0.78 & 0.98 & 1.04 & 0.65 & 0.81 & \\
\hline Indole-3-propionic acid & $\downarrow$ & 0.24 & 0.12 & 0.30 & 0.06 & 0.07 & 0.42 & \\
\hline Glycerol, lipid fraction & $\downarrow$ & 0.64 & 0.68 & 0.83 & 0.85 & 0.77 & 0.88 & \multirow[t]{9}{*}{ Complex lipids, fatty acids and related } \\
\hline Glycerol, polar fraction & $\downarrow$ & 0.94 & 0.81 & 0.89 & 0.91 & 0.94 & 0.92 & \\
\hline dihomo-gamma-Linolenic acid (C20:cis $[8,11,14] 3)$ & $\downarrow$ & 0.72 & 1.02 & 0.88 & 0.92 & 0.78 & 0.79 & \\
\hline Sphingomyelin (d18:2,C16:0) & $\uparrow$ & 1.04 & 1.17 & 1.27 & 1.17 & 0.97 & 1.11 & \\
\hline Sphingomyelin (d18:2,C18:0) & $\uparrow$ & 1.08 & 1.19 & 1.15 & 1.01 & 1.31 & 0.79 & \\
\hline TAG (C16:0,C18:2) & $\downarrow$ & 0.77 & 0.92 & 0.91 & 0.87 & 0.86 & 0.83 & \\
\hline TAG (C18:1,C18:2) & $\downarrow$ & 0.59 & 0.71 & 0.82 & 0.83 & 0.87 & 0.87 & \\
\hline Lysophosphatidylcholine (C20:4) & $\downarrow$ & 1.03 & 0.91 & 0.92 & 0.82 & 0.89 & 0.90 & \\
\hline TAG $(\mathrm{C} 18: 2, \mathrm{C} 18: 3)$ & $\downarrow$ & 0.46 & 0.62 & 0.90 & 0.71 & 0.65 & 0.75 & \\
\hline Lactate & $\downarrow$ & 0.75 & 0.83 & 0.69 & 1.02 & 0.71 & 0.85 & Energy metabolism and related \\
\hline Normetanephrine & $\downarrow$ & 0.69 & 0.66 & 0.88 & 0.88 & 0.65 & 0.62 & \multirow[t]{2}{*}{ Hormones, signal substances and related } \\
\hline 3-O-Methyldopamine & $\downarrow$ & 0.48 & 0.23 & 0.31 & 0.75 & 0.70 & 0.73 & \\
\hline Putrescine & $\downarrow$ & 0.87 & 0.86 & 0.86 & 1.00 & 0.81 & 0.89 & \multirow[t]{2}{*}{ Miscellaneous } \\
\hline Hippuric acid & $\downarrow$ & 0.45 & 0.27 & 0.20 & 0.54 & 0.12 & 0.17 & \\
\hline
\end{tabular}

Figure 2 Heatmap of statistically significantly increased and decreased values of metabolite changes after treatment with aminoglycosides. Metabolite concentration changes in plasma of male $\mathrm{Crl} / \mathrm{Wi}(\mathrm{Han}) \mathrm{rats}(\mathrm{N}=5$ per group) dosed with neomycin sulfate $(250 \mathrm{mg} / \mathrm{kg} \mathrm{bw} / \mathrm{d})$ or gentamicin sulfate $(100 \mathrm{mg} / \mathrm{kg}$ bw/d) for 28 days. Blood samples were taken on study days 7, 14 and 28 after overnight fasting: Figures in red boxes are statistically significantly increased and those in blue boxes statistically significantly decreased metabolite value changes compared to controls ( $\mathrm{N}=10$ per group). Metabolites are mentioned which were statistically significantly altered in the same direction at least at two sampling dates (Welch $t$ test; $\mathrm{p}<0.2$ ) per compound.

\section{Fluoroquinolones}

With 29 significant changes, the plasma metabolite pattern of fluoroquinolones showed the highest number of altered metabolite values compared to the patterns of aminoglycosides and tetracyclines, respectively. Fifteen significantly decreased and fourteen significantly increased metabolite changes were found compared to the control group (see Fig. 3). Changes were seen in amino acids and related, carbohydrates, complex lipids, fatty acids and related, hormones, signal substances and related, miscellaneous, nucleobases and related as well as vitamins, cofactors and related. Over all time points (day 7, 14, 28) hippuric acid was remarkably decreased for both fluoroquinolones tested. The relative abundance in plasma ranged from 0.10 to 0.30 (levofloxacin) and 0.17 to 0.43 (moxifloxacin). Indole-3-acetic acid showed decreased relative abundance in plasma (around 0.5 ) over all time points except on day 28 for levofloxacin with 0.90 . Low relative abundance in plasma could be found for metanephrine. On day 28 relative abundance in plasma twice as high as compared to the control group were found for complex lipids or fatty acids, like 4-hydroxysphinganine, nervonic acid, 3-O-methylsphingosine and sphingomyelin. The relative abundances in plasma of 3-methoxytyrosine and progesterone were up to 10 times higher than control but with a high variation between the three time points, especially day 28. High ratios were observed for coenzymes Q9 and Q10. 
Altogether theses metabolites were more strongly affected after the treatment with moxifloxacin compared to levofloxacin.

\begin{tabular}{|c|c|c|c|c|c|c|c|c|}
\hline \multirow[t]{2}{*}{ Metabolite } & \multirow[t]{2}{*}{ Direction } & \multicolumn{3}{|c|}{ Levofloxacin } & \multicolumn{3}{|c|}{ Moxifloxacin hydrochloride } & \multirow[t]{2}{*}{ Metabolite class } \\
\hline & & $7 \mathrm{~d}$ & $14 \mathrm{~d}$ & $28 \mathrm{~d}$ & $7 \mathrm{~d}$ & $14 \mathrm{~d}$ & $28 \mathrm{~d}$ & \\
\hline Taurine & $\uparrow$ & 1.19 & 1.50 & 1.21 & 1.07 & 1.27 & 1.24 & \multirow[t]{5}{*}{ Amino acids } \\
\hline Glutamine & $\downarrow$ & 0.82 & 0.79 & 0.75 & 0.68 & 0.61 & 0.67 & \\
\hline Valine & $\downarrow$ & 0.96 & 0.85 & 0.87 & 0.89 & 0.94 & 0.96 & \\
\hline Glutamate & $\downarrow$ & 0.76 & 1.07 & 0.77 & 0.72 & 0.93 & 1.00 & \\
\hline Isoleucine & $\downarrow$ & 1.05 & 0.89 & 0.80 & 0.93 & 0.91 & 1.07 & \\
\hline 5-Oxoproline & $\downarrow$ & 0.89 & 0.83 & 0.80 & 0.71 & 0.66 & 0.77 & \multirow[t]{3}{*}{ Amino acids related } \\
\hline Indole-3-acetic acid & $\downarrow$ & 0.41 & 0.52 & 0.90 & 0.55 & 0.63 & 0.63 & \\
\hline Ketoleucine & $\downarrow$ & 0.77 & 0.81 & 0.50 & 0.69 & 0.68 & 0.50 & \\
\hline Glucose & $\uparrow$ & 1.27 & 1.11 & 1.04 & 1.37 & 1.21 & 0.94 & \multirow[t]{2}{*}{ Carbohydrates and related } \\
\hline Xylitol & $\uparrow$ & 1.68 & 1.04 & 1.42 & 1.95 & 1.62 & 0.94 & \\
\hline 4-Hydroxysphinganine (t18:0, Phytosphingosine), total & $\uparrow$ & 1.29 & 1.42 & 1.72 & 1.58 & 1.83 & 2.28 & \multirow{8}{*}{$\begin{array}{l}\text { Complex lipids, fatty acids and } \\
\text { related }\end{array}$} \\
\hline Nervonic acid (C24:cis[15]1) & $\uparrow$ & 0.94 & 1.17 & 1.21 & 1.27 & 1.66 & 2.23 & \\
\hline Cholesterol, total & $\uparrow$ & 0.88 & 1.28 & 1.35 & 0.85 & 1.35 & 1.66 & \\
\hline 3-O-Methylsphingosine (d18:1) & $\uparrow$ & 0.79 & 1.13 & 1.85 & 1.20 & 1.68 & 2.49 & \\
\hline Glycerol, polar fraction & $\downarrow$ & 0.92 & 0.97 & 0.67 & 0.71 & 0.80 & 0.88 & \\
\hline Sphingomyelin (d18:2,C18:0) & $\uparrow$ & 1.01 & 1.32 & 1.27 & 1.47 & 1.51 & 2.61 & \\
\hline TAG (C18:2,C18:3) & $\downarrow$ & 0.42 & 0.48 & 1.27 & 0.65 & 0.99 & 1.31 & \\
\hline Phosphatidylcholine (C18:0,C18:1) & $\uparrow$ & 0.99 & 1.13 & 1.19 & 1.08 & 1.19 & 1.17 & \\
\hline Metanephrine & $\downarrow$ & 0.67 & 0.84 & 0.53 & 0.38 & 0.37 & 0.58 & \multirow{2}{*}{$\begin{array}{l}\text { Hormones, signal substances and } \\
\text { related }\end{array}$} \\
\hline 3-Methoxytyrosine & $\uparrow$ & 1.10 & 1.05 & 1.39 & 1.57 & 1.80 & 2.58 & \\
\hline Hippuric acid & $\downarrow$ & 0.30 & 0.10 & 0.12 & 0.21 & 0.17 & 0.43 & Miscellaneous \\
\hline Cytosine & $\downarrow$ & 0.80 & 0.83 & 0.94 & 0.73 & 0.70 & 0.75 & Nucleobases and related \\
\hline Coenzyme Q10 & $\uparrow$ & 1.41 & 1.31 & 1.73 & 1.42 & 3.04 & 2.28 & \multirow[t]{4}{*}{ Vitamins, cofactors and related } \\
\hline alpha-Tocopherol & $\uparrow$ & 1.00 & 1.34 & 1.47 & 1.57 & 1.44 & 1.24 & \\
\hline Pantothenic acid & $\downarrow$ & 0.85 & 0.77 & 0.67 & 0.52 & 0.69 & 0.73 & \\
\hline Coenzyme Q9 & $\uparrow$ & 0.93 & 1.12 & 1.47 & 1.66 & 2.91 & 2.56 & \\
\hline
\end{tabular}

Figure 3 Heatmap of statistically significantly increased and decreased values of metabolite changes after treatment with fluoroquinolones. Metabolite concentration changes relative to controls in plasma of male $\mathrm{Crl} / \mathrm{Wi}(\mathrm{Han})$ rats $(\mathrm{N}=5$ per group) dosed with levofloxacin $(200 \mathrm{mg} / \mathrm{kg}$ bw/d) or moxifloxacine hydrochloride ( $750 \mathrm{mg} / \mathrm{kg}$ bw/d) for 28 days. Blood samples were taken on study days 7,14 and 28 after overnight fasting: figures in red boxes are statistically signifi- cantly increased and those in blue boxes statistically significantly decreased metabolite value changes compared to controls $(\mathrm{N}=10$ per group). Metabolites are mentioned which were statistically significantly altered in the same direction at least at two sampling dates (Welch $t$ test; $p<0.2$ ) per compound.

\section{Tetracyclines}

After treatment with tetracyclines 13 significantly decreased and 9 significantly increased metabolite changes were found compared to the control group (see Fig. 4). Changes were seen in amino acids and related, carbohydrates, complex lipids, fatty acids and related, hormones, signal substances and related, miscellaneous, nucleobases and related as well as vitamins, cofactors and related. At all three time points (day 7, 14, 28) and for both tetracyclines tested hippuric acid was remarkably decreased. The relative abundance in plasma was between 0.09-0.15 (doxycycline) and 0.16-0.35 (tetracycline). The decreased relative abundance in plasma of indole-3-acetic acid ranged from 0.47 to 0.86 . For both substances the relative abundance in plasma for 3-indoxylsulfate was around 0.5 over all time points. Low relative abundance in plasma, $0.53-0.83$, could be found for metanephrine for both substances. On day 28 4-hydroxysphinganine showed almost doubled values compared to control with a time-dependent increase. The value of 3methoxythyrosine was 1.6 on day 28 for both antibiotics. Cytosine relative abundance in plasma was slightly lower for tetracycline, while coenzyme Q9 values were higher for doxycycline.

The significantly increased or decreased metabolites generally belonged to the same metabolite classes with the exception of the aminoglycoside's pattern. Here, lactate as 


\section{Chapter 2}

metabolite of the energy metabolism was affected but not from the metabolite class of carbohydrates, nucleobases, vitamins or cofactors (see Table 3).

\begin{tabular}{|c|c|c|c|c|c|c|c|c|}
\hline \multirow[t]{2}{*}{ Metabolite } & \multirow[t]{2}{*}{ Direction } & \multicolumn{3}{|c|}{ Doxycycline monohydrate } & \multicolumn{3}{|c|}{ Tetracycline hydrochloride } & \multirow[t]{2}{*}{ Metabolite class } \\
\hline & & $7 \mathrm{~d}$ & $14 \mathrm{~d}$ & $28 \mathrm{~d}$ & $7 \mathrm{~d}$ & $14 \mathrm{~d}$ & $28 \mathrm{~d}$ & \\
\hline Glutamine & $\downarrow$ & 0.81 & 0.76 & 0.83 & 0.76 & 0.75 & 0.62 & \multirow[t]{3}{*}{ Amino acids } \\
\hline Serine & $\uparrow$ & 1.03 & 1.19 & 1.22 & 1.05 & 1.10 & 1.26 & \\
\hline Glutamate & $\uparrow$ & 0.92 & 1.13 & 1.23 & 1.32 & 1.14 & 1.49 & \\
\hline Citrulline & $\downarrow$ & 0.91 & 0.90 & 0.89 & 0.91 & 0.88 & 0.91 & \multirow[t]{5}{*}{ Amino acids related } \\
\hline 5-Oxoproline & $\downarrow$ & 0.79 & 0.84 & 0.88 & 0.80 & 0.81 & 0.72 & \\
\hline Indole-3-acetic acid & $\downarrow$ & 0.47 & 0.52 & 0.64 & 0.67 & 0.74 & 0.86 & \\
\hline Ketoleucine & $\downarrow$ & 0.58 & 0.75 & 0.59 & 0.75 & 0.61 & 0.53 & \\
\hline 3-Indoxylsulfate & $\downarrow$ & 0.68 & 0.45 & 0.25 & 0.33 & 0.47 & 0.51 & \\
\hline Xylitol & $\uparrow$ & 1.51 & 1.05 & 1.97 & 1.04 & 1.16 & 1.86 & \multirow[t]{2}{*}{ Carbohydrates and related } \\
\hline scyllo-Inositol & $\uparrow$ & 1.21 & 1.58 & 1.48 & 1.24 & 1.28 & 1.67 & \\
\hline Tricosanoic acid (C23:0) & $\uparrow$ & 1.04 & 1.26 & 1.46 & 1.10 & 1.36 & 1.31 & \multirow[t]{4}{*}{ Complex lipids, fatty acids and related } \\
\hline 4-Hydroxysphinganine ( $\mathrm{t} 18: 0$, Phytosphingosine), total & $\uparrow$ & 1.33 & 1.57 & 1.71 & 1.37 & 1.59 & 1.81 & \\
\hline Palmitoleic acid (C16:cis[9]1) & $\downarrow$ & 0.82 & 0.59 & 1.03 & 0.71 & 0.70 & 0.83 & \\
\hline Glycerol, polar fraction & $\downarrow$ & 0.71 & 0.78 & 0.80 & 0.74 & 0.75 & 0.75 & \\
\hline Metanephrine & $\downarrow$ & 0.53 & 0.59 & 0.58 & 0.75 & 0.58 & 0.83 & \multirow[t]{2}{*}{ Hormones, signal substances and related } \\
\hline 3-Methoxytyrosine & $\uparrow$ & 1.33 & 1.28 & 1.61 & 1.11 & 1.34 & 1.62 & \\
\hline Spermidine & $\downarrow$ & 0.83 & 0.87 & 0.76 & 0.71 & 0.90 & 0.87 & \multirow[t]{2}{*}{ Miscellaneous } \\
\hline Hippuric acid & $\downarrow$ & 0.15 & 0.09 & 0.10 & 0.35 & 0.19 & 0.16 & \\
\hline Cytosine & $\downarrow$ & 0.75 & 0.89 & 0.84 & 0.59 & 0.57 & 0.49 & \multirow[t]{3}{*}{ Nucleobases and related } \\
\hline Uracil & $\downarrow$ & 0.87 & 0.81 & 1.00 & 0.77 & 0.79 & 0.80 & \\
\hline Allantoin & $\uparrow$ & 1.16 & 1.10 & 0.98 & 1.12 & 1.07 & 1.12 & \\
\hline Coenzyme Q9 & $\uparrow$ & 1.44 & 1.78 & 1.76 & 1.46 & 1.21 & 1.30 & Vitamins, cofactors and related \\
\hline
\end{tabular}

Figure 4 Heatmap of statistically significantly increased and decreased values of metabolite changes after treatment with tetracyclines. Metabolite concentration changes relative to controls in plasma of male $\mathrm{Crl} / \mathrm{Wi}(\mathrm{Han})$ rats $(\mathrm{N}=5$ per group) dosed with doxycy- cline monohydrate $(250 \mathrm{mg} / \mathrm{kg} \mathrm{bw} / \mathrm{d})$ or tetracycline hydrochloride $(50,000 \mathrm{ppm} / \mathrm{d})$ for 28 days. Blood samples were taken on study days

7, 14 and 28 after overnight fasting: figures in red boxes are statisti-cally significantly increased and those in blue boxes statistically sig- nificantly decreased metabolite value changes compared to controls $(\mathrm{N}=10$ per group). Metabolites are mentioned which were statisti- cally significantly altered in the same direction at least at two sampling dates (Welch $\mathrm{t}$ test; $\mathrm{p}<0.2$ ) per compound. 
Table 3 Statistically significantly increased and decreased metabolites changes after treatment with antibiotics of three different classes. Metabolite concentration changes in plasma of male $\mathrm{Crl}: \mathrm{Wi}(\mathrm{Han})$ rats $(\mathrm{N}=5$ per group) dosed with aminoglycosides, fluoroquinolones and tetracyclines for 28 days. $\uparrow=$ metabolite level is increased in at least two of the three sampling dates; $\downarrow=$ metabolite level is decreased in at least two of the three sampling dates; metabolite values were compared to controls $(\mathrm{N}=$ 10 per group; Welch-t-test; $p<0.2$ ).

\begin{tabular}{|c|c|c|c|c|}
\hline Metabolite class & Metabolite & Aminoglycosides & Fluoroquinolones & Tetracyclines \\
\hline \multirow[t]{9}{*}{ Amino acids } & Alanine & $\downarrow$ & & \\
\hline & Glutamate & & $\downarrow$ & $\uparrow$ \\
\hline & Glutamine & & $\downarrow$ & $\downarrow$ \\
\hline & Isoleucine & & $\downarrow$ & \\
\hline & Proline & $\downarrow$ & & \\
\hline & Serine & & & $\uparrow$ \\
\hline & Taurine & & $\uparrow$ & \\
\hline & Tyrosine & $\downarrow$ & & \\
\hline & Valine & & $\downarrow$ & \\
\hline \multirow[t]{6}{*}{ Amino acids related } & 3-Indoxylsulfate & & & $\downarrow$ \\
\hline & 5-Oxoproline & & $\downarrow$ & $\downarrow$ \\
\hline & Citrulline & & & $\downarrow$ \\
\hline & Indole-3-acetic acid & $\downarrow$ & $\downarrow$ & $\downarrow$ \\
\hline & Indole-3-propionic acid & $\downarrow$ & & \\
\hline & Ketoleucine & $\downarrow$ & $\downarrow$ & $\downarrow$ \\
\hline \multirow{3}{*}{$\begin{array}{l}\text { Carbohydrates and } \\
\text { related }\end{array}$} & Glucose & & $\uparrow$ & \\
\hline & scyllo-Inositol & & & $\uparrow$ \\
\hline & Xylitol & & $\uparrow$ & $\uparrow$ \\
\hline \multirow{15}{*}{$\begin{array}{l}\text { Complex lipids, fatty } \\
\text { acids and related }\end{array}$} & 3-O-Methylsphingosine (d18:1) & & $\uparrow$ & \\
\hline & 4-Hydroxysphinganine (t18:0, Phytosphingosine), total & & $\uparrow$ & $\uparrow$ \\
\hline & Cholesterol, total & & $\uparrow$ & \\
\hline & dihomo-gamma-Linolenic acid (C20:cis[8,11,14]3) & $\downarrow$ & & \\
\hline & Glycerol, polar fraction & $\downarrow$ & $\downarrow$ & $\downarrow$ \\
\hline & Lysophosphatidylcholine (C20:4) & $\downarrow$ & & \\
\hline & Nervonic acid (C24:cis[15]1) & & $\uparrow$ & \\
\hline & Palmitoleic acid (C16:cis[9]1) & & & $\downarrow$ \\
\hline & Phosphatidylcholine (C18:0,C18:1) & & $\uparrow$ & \\
\hline & Sphingomyelin (d18:2,C16:0) & $\uparrow$ & & \\
\hline & Sphingomyelin (d18:2,C18:0) & $\uparrow$ & $\uparrow$ & \\
\hline & TAG (C16:0,C18:2) & $\downarrow$ & & \\
\hline & TAG (C18:1,C18:2) & $\downarrow$ & & \\
\hline & TAG (C18:2,C18:3) & $\downarrow$ & $\downarrow$ & \\
\hline & Tricosanoic acid (C23:0) & & & $\uparrow$ \\
\hline $\begin{array}{l}\text { Energy metabolism and } \\
\text { related }\end{array}$ & Lactate & $\downarrow$ & & \\
\hline \multirow{4}{*}{$\begin{array}{l}\text { Hormones, signal } \\
\text { substances and related }\end{array}$} & 3-Methoxytyrosine & & $\uparrow$ & $\uparrow$ \\
\hline & 3-O-Methyldopamine & $\downarrow$ & & \\
\hline & Metanephrine & & $\downarrow$ & $\downarrow$ \\
\hline & Normetanephrine & $\downarrow$ & & \\
\hline \multirow[t]{3}{*}{ Miscellaneous } & Hippuric acid & $\downarrow$ & $\downarrow$ & $\downarrow$ \\
\hline & Putrescine & $\downarrow$ & & \\
\hline & Spermidine & & & $\downarrow$ \\
\hline \multirow{3}{*}{ Nucleobases and related } & Allantoin & & & $\uparrow$ \\
\hline & Cytosine & & $\downarrow$ & $\downarrow$ \\
\hline & Uracil & & & $\downarrow$ \\
\hline \multirow{4}{*}{$\begin{array}{l}\text { Vitamins, cofactors and } \\
\text { related }\end{array}$} & alpha-Tocopherol & & $\uparrow$ & \\
\hline & Coenzyme Q9 & & $\uparrow$ & $\uparrow$ \\
\hline & Coenzyme Q10 & & $\uparrow$ & \\
\hline & Pantothenic acid & & $\downarrow$ & \\
\hline
\end{tabular}




\section{DISCUSSION}

\section{Metabolome pattern for each class of antibiotics}

In our studies we demonstrate that antibiotics have a profound influence on the plasma metabolites in rats following oral administration. Aminoglycosides have, in contrast to the other antibiotics, poor activity against obligate anaerobic and most gram-positive bacteria (except for most staphylococci), but are active against most gram-negative aerobic or facultative anaerobic bacilli (Brown and Riviere 1991; Dortantz and Devices 1999; Mingeot-Leclercq et al. 1999). The antimicrobial spectrum of the third-generation levofloxacin and fourth-generation moxifloxacin includes aerobic and facultative anaerobic Gram-positive and most Gram-negative organisms, with additional activity of moxifloxacin against most clinically significant obligates anaerobes (Scoper 2008). Tetracyclines have a bacteriostatic activity against almost all medically relevant aerobic and anaerobic bacterial genera, both Gram-positive and Gram-negative, mycoplasmas, rickettsiae, chlamydiae, and even some protozoa (Boothe 2015). Here we show that there are antibiotic class specific changes in the plasma metabolome of rats: for each class of antibiotics specific metabolome patterns could be established. This could enable an identification of new compounds or biologicals with an antibiotic activity and associate the metabolome change with a specific class of antibiotics. The second purpose of these investigations was to ascertain which of the metabolites obtained in the plasma from rats are associated with microbiome functionality.

The pattern of aminoglycosides (neomycin and gentamicin) with 17 decreased metabolites, while two significantly increased, is considerably different than the other patterns obtained with fluoroquinolones (levofloxacin and moxifloxacin) and tetracyclines (tetracycline and doxycycline). The particular importance of the aminoglycosides for this research is the fact that they are very poorly absorbed from the gastrointestinal tract. Numerical values for absorption range from $1-3 \%$, the highest mentioned for rats is $6 \%$ (Renshaw et al.; Brown and Riviere 1991; Bergogne-Bérézin and Bryskier 1999; Dhillon 2006). If we assume that $6 \%$ can be absorbed, we would obtain a systemic dose level of $15 \mathrm{mg} / \mathrm{kg}$ body weight for neomycin and $6 \mathrm{mg} / \mathrm{kg}$ body weight for gentamicin. Compared to safe human therapeutic dose levels (gentamicin up to $7 \mathrm{mg} / \mathrm{kg}$ IV every 24 hours for 7 to 21 days (Drugs.com [Internet, 2016a]; European Medicines Agency 2016), neomycin orally up to 100 $\mathrm{mg} / \mathrm{kg} /$ day divided every 6 hours with a treatment period of 2 weeks (Drugs.com [Internet], 2016b) we can virtually exclude that systemic toxicity would have contributed significantly to the metabolite changes detected with these two compounds.

This conclusion is in line with several studies in rats; the neomycin-NOAEL of a two-year oral carcinogenicity study with rats being $25 \mathrm{mg} / \mathrm{kg}$ bw/day and the same value being reported in a multi-generation reproductive and teratogenicity study in rats (van KotenVermeulen and van Leeuwen 1995). Oral dosing of gentamicin in rats for a period of 13 weeks up to doses of $116 \mathrm{mg} / \mathrm{kg}$ bw/day showed no treatment-related effects in kidneys, 
liver, heart and brain even at the highest dose level. The NOEL was $19 \mathrm{mg} / \mathrm{kg} \mathrm{bw} / \mathrm{day}$ (Roberts 1995).

Therefore, the changes in the plasma profile detected should not be caused by systemic effects (i.e. organ toxicity) and consequently, plasma metabolome changes caused by these antibiotics are highly likely the result of their effect on microbiome functionality. Thus, these results are a key component for the evaluation of microbiome specific effects. Compared to the other two patterns, 13 out of the 19 altered metabolites are unique for the aminoglycoside pattern (see Table 3).

With 26 significantly lower or higher values, the metabolite pattern of fluoroquinolones showed the highest number of altered metabolite values compared to the patterns of aminoglycosides and tetracyclines. Increased relative abundance in plasma for 4hydroxysphinganine, nervonic acid, 3-O-methylsphingosine, sphingomyelin, coenzymes Q9 and Q10 were more pronounced for moxifloxacin. Moxifloxacin is an antibiotic of the fourth generation with a broader activity spectrum and thus is more effective against Grampositive bacteria and anaerobes than levofloxacin (Scoper 2008). This could explain the potentiation of the effect reflected by the higher relative abundance values in plasma for moxifloxacin than levofloxacin.

After treatment with tetracyclines 22 significantly altered values of metabolite changes were found (see Table 3). On day 28 after tetracycline treatment values of 4hydroxysphinganine were almost doubled compared to control and a time-dependent increase could be observed. Cytosine relative abundance in plasma were slightly lower for tetracycline, while coenzyme Q9 values were higher for doxycycline.

In contrast to the aminoglycoside pattern, the number of increased and decreased metabolites are similar after treatment with tetracyclines or fluoroquinolones.

\section{Identification of microbiota-derived metabolites}

Since it can be assumed that aminoglycosides do not have a systemic effect in this study, changes of the metabolites were probably due to an altered microbiome (see Table 3). All metabolites, except two sphingomyelin isomers, were decreased. Besides hippuric acid and indole-3-acetic acid, the metabolite indole-3-propionic acid, which belongs to the tryptophan and indole metabolic pathway, was found to be a unique biomarker metabolite for the aminoglycoside pattern. IPA is a potent antioxidant and because of its neuroprotective property it is being studied for therapeutic use in Alzheimer's disease (Chyan et al. 1999; Paul E. Bendheim, Burkhard Poeggeler, Eyal Neria, Vivi Ziv 2002). Since IPA could not be found in the plasma of germ-free mice in contrast to conventional mice, Wikoff et al. colonized them with Clostridium sporogenes ATCC 15579 (Wikoff et al. 2009). IPA was observed five days after colonization which demonstrates that the continuous IPA production from one bacterial species in the gastrointestinal tract is 
sufficient to introduce IPA into the bloodstream of the host (Wikoff et al. 2009). However, C. sporegenes is an obligate anaerobe and hence should not be affected strongly by aminoglycosides. It could be speculated that either this strain is affected over the long time of the treatment or there are other strains that are able to produce enough IPA at levels, which are subsequently detectable in the serum.

Three metabolites, of which relative abundances in plasma were decreased in all patterns, could be identified as key metabolites in all three patterns: indole-3-acetic acid, hippuric acid and glycerol. Since they could be found in the aminoglycoside pattern, it is highly likely that these metabolites are derived from the microbiota. We suggest that if these metabolites were derived from the diet, the metabolite values would not be altered.

Indole-3-acetic acid, a protein-bound uremic solute, is generated either from bacterial indole production or from dietary tryptophan degradation by both endogenous and bacterial cells, e.g. Clostridium sporogenes, Clostridium bartlettii and E coli (Ramezani et al. 2015). IAA contributes to the progression of CKD in rats (Satoh et al. 2003), and in serum of CKD patients IAA is a significant predictor of mortality and cardiovascular events, inducing proinflammatory enzyme COX-2 and oxidative stress via the nongenomic AhR pathway (Dou et al. 2014). In antibiotic-treated rats, the tryptophan metabolism appeared to be significantly altered because levels of indole-containing substances like 3-indoxylsulfate and IAA were decreased both in urine and feces whereas tryptophan and tryptamine levels were increased significantly in feces (Zheng et al. 2011).

Also, a well-known urinary metabolite of host-microbial origin is hippuric acid and its concentrations can be modulated by diet, stress, disease, and the microbiome (Phipps et al. 1998; Nicholson et al. 2012). The microbes produce benzoate by metabolizing aromatic compounds and polyphenols, and benzoate is then conjugated with glycine in the liver, kidney and intestine (Strahl and Barr 1971; Chiba et al. 1994; Poon and Pang 1995). Related bacteria are Clostridium difficile, F. prausnitzii, Bifidobacterium, Subdoligranulum, Lactobacillus (Strahl and Barr 1971; Chiba et al. 1994; Poon and Pang 1995; Nicholson et al. 2012). HA is associated to a variety of health and disease states: it may be a biomarker of hypertension and obesity in humans, may cause an increase of anion gap acidosis and glucose intolerance, interferes with erythropoiesis and platelet cyclooxygenase activity (Remer and Manz 2003; Li et al. 2008; Kohli et al. 2011; Ramezani et al. 2015). Bacterial suppression by antibiotics reduces the excretion of hippuric acid in blood, urine and feces of rodents (Williams et al. 2002; Swann et al. 2011; Zheng et al. 2011). Also in germ-free animals the plasma and urinary concentrations of hippuric acid are reduced (Nicholls et al. 2003; Wikoff et al. 2009). 
Table 4 Potential microbiome-derived metabolites: statistically significantly increased and decreased metabolites changes after treatment with nonabsorbable aminoglycosides. Metabolite concentration changes in plasma of male $\mathrm{Crl}: \mathrm{Wi}(\mathrm{Han})$ rats $(\mathrm{N}=5$ per group) dosed with neomycin and gentamicin sulfate; $\uparrow=$ metabolite level is increased in at least two of the three sampling dates; $\downarrow=$ metabolite level is decreased in at least two of the three sampling dates; metabolite values were compared to controls $(\mathrm{N}=10$ per group; Welch-t-test; $\mathrm{p}<$ $0.2)$.

\begin{tabular}{lcl}
\hline Metabolite & Direction & Metabolite class \\
& & \\
\hline Alanine & $\downarrow$ & Amino acids \\
Proline & $\downarrow$ & \\
Tyrosine & $\downarrow$ & \\
\hline Indole-3-acetic acid & $\downarrow$ & Amino acids related \\
Ketoleucine & $\downarrow$ & \\
Indole-3-propionic acid & $\downarrow$ & \\
\hline Glycerol & $\downarrow$ & Complex lipids, fatty acids and related \\
dihomo-gamma-Linolenic acid (C20:cis[8,11,14]3) & $\downarrow$ & \\
Sphingomyelin (d18:2,C16:0) & $\uparrow$ & \\
Sphingomyelin (d18:2,C18:0) & $\uparrow$ & \\
TAG (C16:0,C18:2) & $\downarrow$ & \\
TAG (C18:1,C18:2) & $\downarrow$ & \\
Lysophosphatidylcholine (C20:4) & $\downarrow$ & \\
TAG (C18:2,C18:3) & $\downarrow$ & \\
\hline Lactate & $\downarrow$ & Energy metabolism and related \\
\hline Normetanephrine & $\downarrow$ & Hormones, signal substances and related \\
3-O-Methyldopamine & $\downarrow$ & \\
\hline Putrescine & $\downarrow$ & Miscellaneous \\
Hippuric acid & $\downarrow$ & \\
\hline
\end{tabular}

Through ingredients of the diet, e.g. triacylglycerols and phospholipids, and/or by in situ microbial production or release from desquamated epithelial cells, glycerol can be found in the gastrointestinal tract (De Weirdt et al. 2010). Components of the lipids, like triacylglycerols, phosphatidylcholine, lysophosphatidylcholine, glycerol, myo-inositol and fatty acids, can be measured in the blood (Mellert et al. 2011). Evidence that glycerol is produced by the gut microbiota is provided by an experiment of Frost et al.: they compared human to gelada microbiota and found out that grass stimulated the gelada microbiota to produce ethanol and glycerol while it stimulated the human microbiota to produce acetate and propionate (Frost et al. 2014). In a 28 day study in rats Mellert et al. found that plasma glycerol levels were decreased in male rats under dietary restriction when compared to controls as well as when overnight fasted animals were compared to non-fasted individuals (Mellert et al. 2011). In human feces, a high concentration of glycerol and a reduced amount of butyrate was found in the feces of Crohn's disease patients in comparison with the control group and ulcerative colitis patients, but the origin and role of glycerol in the feces is unclear (Marchesi et al. 2007). Compared to rat feces glycerol seems to be unique to the human (Saric et al. 2008). Glycerol seems to play an important role in the microbiome and we suggest that both dietary restriction and especially a gut bacterial reduction due to antibiotic treatment may lead to the decrease of glycerol levels in the blood of the rats. 


\section{Influence of systemic toxicity on metabolite patterns}

For the tetracycline and fluoroquinolone antibiotics, which are absorbed from the gastrointestinal tract, a possible influence of systemic toxicity must be taken into account when evaluating their effects on the metabolome (see Table 4). Side effects of fluoroquinolones in rats and/or humans can involve the blood, heart, liver, kidney, central nervous system (CNS), thyroid and oculotoxicity, and adverse effects of tetracyclines were seen in the adrenals, liver, bones, kidney, testes, thyroid heart, inner ear, CNS and on the skin (phototoxicity) (Adir 1975; Vargas-Estrada et al. 2008; U.S. Food and Drug Administration 2013; Musther et al. 2014; U.S. Food and Drug Administration 2015). The dose levels selected for the tetracycline and fluoroquinolone antibiotics were below the maximum tolerated dose for rats, but in a dose-range in which systemic toxicity cannot be excluded.

In this study, several metabolites that were not significantly altered after the treatment with aminoglycosides and could be either produced by the microbes, the mammalian cells or were degraded from diet, are discussed in the following.

Table 5 Classes of antibiotics, mechanism of action, main side effects and bioavailability of neomycin, streptomycin, tetracycline, doxycycline, levofloxacin and moxifloxacin.

\begin{tabular}{|c|c|c|c|c|}
\hline Class of antibiotics & Mechanism of action & $\begin{array}{l}\text { Side effects } \\
\text { (rats) }\end{array}$ & $\begin{array}{l}\text { Side effects } \\
\text { (humans) }\end{array}$ & $\begin{array}{l}\text { Bioavailability } \\
\text { (rats) }\end{array}$ \\
\hline Aminoglycosides & $\begin{array}{l}\text { inhibition of protein synthesis } \\
\text { bacteriostatic }\end{array}$ & kidney, inner ear & kidney, inner ear & $6 \%$ (gentamicin) $^{2}$ \\
\hline Tetracyclines & $\begin{array}{l}\text { inhibition of protein synthesis } \\
\text { bacteriostatic }\end{array}$ & $\begin{array}{l}\text { adrenals, bones, CNS, } \\
\text { kidney, liver, testes, } \\
\text { thyroid }\end{array}$ & $\begin{array}{l}\text { kidney, heart, liver, inner } \\
\text { ear, CNS, phototoxicity }\end{array}$ & $80-100 \%^{b}$ \\
\hline Fluoroquinolones & $\begin{array}{l}\text { preventing bacterial DNA from } \\
\text { unwinding and duplicating } \\
\text { bacericidal }\end{array}$ & $\begin{array}{l}\text { CNS, heart, kidney, } \\
\text { liver, testes, thyroid }\end{array}$ & $\begin{array}{l}\text { blood, CNS, heart, kidney, } \\
\text { liver, phototoxicity, bones } \\
\text { (arthropathy), oculotoxicity }\end{array}$ & $80 \%{ }^{\mathrm{c}}$ \\
\hline
\end{tabular}

${ }^{a}$ (Renshaw et al.; Brown and Riviere 1991; Bergogne-Bérézin and Bryskier 1999; Dhillon 2006)

b(Adir 1975; Vargas-Estrada et al. 2008)

'(von Keutz and Schlüter 1999; Musther et al. 2014; Ahmadi et al. 2016)

After tetracycline treatment serum levels for 3-indoxylsulfate (IS), which is a metabolite in the tryptophan and indole metabolic pathway, were reduced by around 50 percent. Tryptophan either derives from protein degradation from food or can be synthesized by the gut microbiota (Clarke et al. 2014; Heinken and Thiele 2015b). The bacterial tryptophanase can degrade tryptophan to skatole and indole, respectively. From the intestine the latter is further absorbed into the blood and metabolized in the liver via phase II transformation to indoxylsulfate (Zheng et al. 2011; Heinken and Thiele 2015b). IS is an uremic toxin and directly linked to vascular disease and mortality and progression of chronic kidney disease (Zeng et al.; Barreto et al. 2009; Wu et al. 2011). 
Our results are concordant with other studies where plasma extracts from germ-free mice were compared to samples from conventional mice: IS was identified only in the serum of animals harboring microbes (Wikoff et al. 2009). Also decreased relative abundance in plasma of IS was detected in rat feces and urine after antibiotic treatment due to gut bacterial reduction and subsequently a loss of bacterial enzymes like tryptophanase (Wu et al. 2011; Zheng et al. 2011).

In the metabolite pattern of fluoroquinolones and tetracyclines metanephrine values were decreased and 3-methoxytyrosine and coenzyme Q10 and/or Q9 are upregulated. Coenzyme Q serves as an important redox active constituent in both mitochondria and lipid membranes (Kamp et al. 2012a). This suggests that the mitochondrial membranes may have been affected by tetracyclines and that an increased plasma level of oxidized coenzyme Q9 could finally lead to hepatotoxicity (Kohli et al. 2011).

In both classes, there were also altered values of glutamine and glutamate, which may play an important role in the microbiome. Both metabolites are produced by the microbes and can be used as energy sources to enterocytes, especially during illness and stress, and many studies in the last decades indicated that glutamine is an important metabolite for the smallintestinal mucosal cells regulating many cellular mechanisms (Dai et al. 2013; Davila et al. 2013; Frye et al. 2015; Heinken and Thiele 2015b). In plasma and tissue profiles in murine colitis models altered values of these metabolites were found, which were correlated with the developmental phases of this illness and severity (Holmes et al. 2011; Shiomi et al. 2011). Glutamine can be produced by glutamate and ammonia and in a study of Thomas et al. pretreatment with glutamine prior to surgical gut manipulation in rodents prevents gut and lung injury due to oxidative and inflammatory processes and protects enterocyte mitochondria (Thomas et al. 2005).

Besides hippuric acid, there was a decrease of the polyamines putrescine and spermidine after treatment with aminoglycosides or tetracylines, respectively. They are generated from precursor amino acids by the microbes and in low amounts by normal colonocytes (Davila et al. 2013; Ramezani et al. 2015). They are involved in various physiological processes both in bacterial and endogenous cells, like the biofilm formation, bacteriocin production and the microbial pathogenesis (Tabor and Tabor 1985; Shah and Swiatlo 2008; Igarashi and Kashiwagi 2010). However, none of them was altered after the treatment with fluoroquinolones. 
Table 6 Statistically significantly increased and decreased metabolites after treatment with fluoroquinolones or tetracyclines, respectively. The metabolites changes could originate from systemic/organ toxicity and can be found in different mode of action (MOA) metabolome patterns in the data base MetaMap ${ }^{\circledR}$ Tox.

\begin{tabular}{lll} 
Class of antibiotics & Metabolite & MOA patterns in MetaMap ${ }^{\circledR}$ Tox \\
\hline Fluoroquinolones & 3-O-Methylsphingosine & liver \\
& 3-Methoxytyrosine & CNS, steroid hormones \\
& 4-Hydroxysphinganine & kidney, liver \\
& 5-Oxoproline & testes \\
& Cholesterol & liver, thyroid \\
& Cytosine & kidney \\
Glutamate & kidney \\
Glutamine & testes \\
Glycerol & kidney, steroid hormones \\
& Metanephrine & kidney \\
Nervonic acid & kidney, liver \\
Phosphatidylcholine & liver \\
Taurine & kidney, testes \\
Xylitol & kidney \\
3-Methoxytyrosine & CNS, steroid hormones \\
4-Hydroxysphinganine & kidney, liver \\
5-Oxoproline & testes \\
Allantoin & kidney \\
Cytosine & kidney \\
Glutamine & testes \\
Glycerol & kidney, steroid hormones \\
Metanephrine & kidney \\
Palmitoleic acid & testes, spleen \\
Xylitol & kidney \\
\hline &
\end{tabular}

Various significantly increased and decreased metabolites after the treatment with fluoroquinolones or tetracyclines can be also found in MOA metabolome patterns in Table 5 in the data base MetaMap ${ }^{\circledR}$ Tox. The metabolites and the corresponding MOA patterns are listed in Table 6. The findings suggest that these metabolites could be caused by organ toxicity, especially due to liver, kidney and testes toxicity, which can be found in toxicological studies in the rat (see Table 4). Other MOA patterns, which include the altered metabolites, are related to organ toxicity of the thyroid, the CNS and disturbances of the steroid hormones.

The plasma metabolite profile is dependent on the bactericidal/-static efficacy spectrum of the antibiotic, its bioavailability, and consequently its potential systemic effect. This leads to specific changes in the metabolite pattern and is hence an opportunity to identify and characterize substances that may have antibiotic properties.

Taken together, plasma metabolite changes of hippuric acid, indole-3-acetic acid and glycerol following antibiotic treatment and microbiota changes are highly likely to reflect changes in microbiome-derived metabolites. This could be concluded because aminoglycosides are poorly absorbed from the gut and thus just have an effect on the microbes. Indole-3-propionic acid was found to be a unique biomarker plasma metabolite for the aminoglycosides and 3-indoxylsulfate for the tetracyclines. Besides other amino 
acids and amino acid related metabolites, coenzyme Q, glutamine, glutamate, putrescine and spermidine were found to be mammalian-microbiome co-metabolites.

The results suggest that plasma based metabolic profiling (metabolomics) could be a suitable tool to investigate the effect of compounds on the functionality of the microbiome and to obtain insight in the mammalian-microbiome co-metabolism of endogenous metabolites. The investigation of the production of metabolites absorbed by the host, or microbiome's functionality, is also a concept to assess toxicological risks of new compounds or biologicals associated with microbiota changes. Therefore, it is essential to determine the composition of the intestinal microbiota, organization and the hostmicrobiota interactions. The combination of metabolomics, metagenomics, e.g. 16S rRNA sequencing, and systems biology would be an interdisciplinary approach to identify adverse effects on the microbiota that could lead to disease. Targeted manipulation of the microbiota with antibiotics and subsequent community analyses could enable a correlation between metabolites and bacterial species. Considering toxicological studies in rats, however, it is important first to investigate the "base line variability" of their microbiota analyzing control groups with standardized diet, control groups with corn oil or carboxymethyl cellulose, that are normally used as formulation of test substances. It also includes a control group with restricted diet because the effect of dieteray restriction on the metabolite pattern has been proven and ingestion of less food is likely to cause a shift in the microbial community. These targeted experimental investigations are currently carried out. The specific plasma metabolite patterns are a first step to in the future identify substances in the data base MetaMap ${ }^{\circledR}$ Tox with a similar effect on the microbiome.

Acknowledgement: We would like to thank Ms Irmgard Weber for her skillful assistance.

Funding information: The authors have no relevant affiliations or financial involvement with any organization or entity with a financial interest in or financial conflict with the subject matter or materials discussed in the manuscript. This includes employment, consultancies, honoraria, stock ownership or options, expert testimony, grants or patents received or pending, or royalties. No writing assistance was utilized in the production of this manuscript. 


\section{REFERENCES}

Adir J (1975) Enterohepatic circulation of tetracycline in rats. J Pharm Sci 64:1847-50. doi: 10.1002/jps.2600641121

Ahmadi R, Ahmadifar M, Safarpour E, et al (2016) The Effects of Levofloxacin on Testis Tissue and Spermatogenesis in Rat. Cell J 18:112-116.

Arumugam M, Raes J, Pelletier E, et al (2011) Enterotypes of the human gut microbiome. 1-7. doi: 10.1038/nature09944

Barreto FC, Barreto D V, Liabeuf S, et al (2009) Serum Indoxyl Sulfate Is Associated with Vascular Disease and Mortality in Chronic Kidney Disease Patients. Clin J Am Soc Nephrol 4:1551-1558. doi: 10.2215/CJN.03980609

Beger RD, Sun J, Schnackenberg LK (2010) Metabolomics approaches for discovering biomarkers of drug-induced hepatotoxicity and nephrotoxicity. Toxicol. Appl. Pharmacol. 243:154-166.

Bergogne-Bérézin E, Bryskier a. (1999) The suppository form of antibiotic administration: Pharmacokinetics and clinical application. J Antimicrob Chemother 43:177-185. doi: 10.1093/jac/43.2.177

Boothe DM (2015) The Merck veterinary manual: Tetracyclines.

http://www.merckvetmanual.com/mvm/pharmacology/antibacterial_agents/tetracycli nes.html.

Brown SA, Riviere JE (1991) Comparative pharmacokinetics of amionoglycoside antibiotics. J Vet Pharmacol Ther 14:1-35. doi: 10.3109/14992027.2010.524253

Center for Drug Evaluation and Research (2003) Application number: 21-598.

Center for Drug Evaluation and Research (2013) Application Number: 204063Orig1s000.

Chiba M, Poon K, Hollands J, Pang K (1994) Glycine conjugation activity of benzoic acid and its acinar localization in the perfused rat liver. J Pharmacol Exp Ther 268:40916.

Cho I, Blaser MJ (2012) The human microbiome: at the interface of health and disease. Nat Rev Genet 13:260-70. doi: 10.1038/nrg3182

Chyan YJ, Poeggeler B, Omar RA, et al (1999) Potent neuroprotective properties against the Alzheimer $\beta$-amyloid by an endogenous melatonin-related indole structure, indole-3-propionic acid. J Biol Chem 274:21937-21942. doi: 10.1074/jbc.274.31.21937

Clarke G, Stilling RM, Kennedy PJ, et al (2014) Gut Microbiota: The Neglected Endocrine Organ. Mol Endocrinol 28:1221-1238. doi: 10.1210/me.2014-1108

Dai ZL, Li XL, Xi P Bin, et al (2013) L-Glutamine regulates amino acid utilization by intestinal bacteria. Amino Acids 45:501-512. doi: 10.1007/s00726-012-1264-4

Dalhoff a (2001) Comparative in vitro and in vivo activity of the C-8 methoxy quinolone moxifloxacin and the C-8 chlorine quinolone BAY y 3118. Clin Infect Dis 32 Suppl 1:S16-22. doi: 10.1086/319371

David L a, Maurice CF, Carmody RN, et al (2014) Diet rapidly and reproducibly alters the human gut microbiome. Nature 505:559-63. doi: 10.1038/nature12820 
Davila AM, Blachier F, Gotteland M, et al (2013) Re-print of "intestinal luminal nitrogen metabolism: Role of the gut microbiota and consequences for the host." Pharmacol Res 69:114-126. doi: 10.1016/j.phrs.2013.01.003

De Weirdt R, Possemiers S, Vermeulen G, et al (2010) Human faecal microbiota display variable patterns of glycerol metabolism. FEMS Microbiol Ecol 74:601-611. doi: 10.1111/j.1574-6941.2010.00974.x

Dhillon S (2006) Clinical Pharmacokinetics. Pharmaceutical Press

Dietz D (1989) Toxicology and Carcinogenesis Studies of Tetracycline Hydrochloride. US Dep Heal Hum Serv 344:1-171.

Dortantz PM, Devices M (1999) Comparison of Tobramycin Nephrotoxicity i n Young Adult and Aged Female Rats.

Dou L, Sallée M, Cerini C, et al (2014) The Cardiovascular Effect of the Uremic Solute Indole-3 Acetic Acid. J Am Soc Nephrol 1-12. doi: 10.1681/ASN.2013121283

Drugs.com [Internet, 2016a] Gentamicin Dosage. In: Gentamicin Inf. from drugs.com, Updat. 2016 Sept. https://www.drugs.com/dosage/gentamicin.html. Accessed 14 Sep $2016 \mathrm{a}$

Drugs.com [Internet, 2016b] Neomycin Dosage. In: Neomycin Inf. from drugs.com, Updat. 2016 Sept. https://www.drugs.com/dosage/neomycin.html. Accessed 14 Sep 2016b

European Medicines Agency (2016) European public MRL assessment report ( EPMAR ) European public MRL: Gentamicin.

Frost GS, Walton GE, Swann JR, et al (2014) Impacts of Plant-Based Foods in Ancestral Hominin Diets on the Metabolism and Function of Gut Microbiota In Vitro. 5:1-11. doi: 10.1128/mBio.00853-14.Editor

Frye RE, Rose S, Slattery J, MacFabe DF (2015) Gastrointestinal dysfunction in autism spectrum disorder: the role of the mitochondria and the enteric microbiome. Microb Ecol Health Dis 26:27458.

Guarner F, Malagelada JR (2003) Gut flora in health and disease. Lancet 361:1831. doi: 10.1016/S0140-6736(03)12489-0

Heinken A, Thiele I (2015a) Systems biology of host-microbe metabolomics. WIREs Syst Biol Med 7:195-219. doi: 10.1002/wsbm.1301

Heinken A, Thiele I (2015b) Gut Microbes Systematic prediction of health-relevant humanmicrobial co-metabolism through a computational framework. doi: 10.1080/19490976.2015.1023494

Holmes E, Li J V, Athanasiou T, et al (2011) Understanding the role of gut microbiome host metabolic signal disruption in health and disease. Trends Microbiol 19:349-359. doi: 10.1016/j.tim.2011.05.006

Huse SM, Dethlefsen L, Huber J a., et al (2008) Exploring Microbial Diversity and Taxonomy Using SSU rRNA Hypervariable Tag Sequencing. PLoS Genet 4:e1000255. doi: 10.1371/journal.pgen.1000255

Igarashi K, Kashiwagi K (2010) Modulation of cellular function by polyamines. Int J Biochem Cell Biol 42:39-51. doi: 10.1016/j.biocel.2009.07.009 
Kamp H, Fabian E, Groeters S, et al (2012a) Application of in vivo metabolomics to preclinical/toxicological studies: case study on phenytoin-induced systemic toxicity.

Kamp H, Strauss V, Wiemer J, et al (2012b) Reproducibility and robustness of metabolome analysis in rat plasma of 28-day repeated dose toxicity studies. Toxicol Lett 215:143149.

Kirpich IA, Marsano LS, McClain CJ (2015) Gut-liver axis, nutrition, and non-alcoholic fatty liver disease. Clin. Biochem. 48:923-930.

Kohli R, Kirby M, Xanthakos S a, et al (2011) High-Fructose Medium-Chain-Trans-Fat Diet Induces Liver Fibrosis \& Elevates Plasma Coenzyme Q9 in a Novel Murine Model of Obesity and NASH. 52:934-944. doi: 10.1002/hep.23797.High-Fructose

Kuroda T, Namba K, Torimaru T, et al (2001) Variability of oral bioavailability for low dose methotrexate in rats. Eur J Drug Metab Pharmacokinet 26:227-234.

Lecomte V, Kaakoush NO, Maloney C a., et al (2015) Changes in Gut Microbiota in Rats Fed a High Fat Diet Correlate with Obesity-Associated Metabolic Parameters. PLoS One 10:e0126931. doi: 10.1371/journal.pone.0126931

Li M, Wang B, Zhang M, et al (2008) Symbiotic gut microbes modulate human metabolic phenotypes. Proc Natl Acad Sci U S A 105:2117-2122. doi:

10.1073/pnas.0712038105

Lindon JC, Holmes E, Bollard ME, et al (2004) Metabonomics technologies and their applications in physiological monitoring, drug safety assessment and disease diagnosis. Biomarkers 9:1-31. doi: 10.1080/13547500410001668379

Looser R, Krotzky AJ, Trethewey N (2005) Metabolite profiling with GC-MS and LC-MS - a key tool for contemporary biology. In: Vaidyanathan S, Harrigan G, Goodacre R (eds) Metabolome analyses - Strategies for systems biology. Springer, New York, pp 103-118

Marchesi JR, Holmes E, Khan F, et al (2007) Rapid and noninvasive metabonomic characterization of inflammatory bowel disease. J Proteome Res 6:546-551. doi: 10.1021/pr060470d

Marcobal A, Kashyap PC, Nelson T a, et al (2013) A metabolomic view of how the human gut microbiota impacts the host metabolome using humanized and gnotobiotic mice. ISME J 7:1933-1943. doi: 10.1038/ismej.2013.89

Marcobal A, Yusufaly T, Higginbottom S, et al (2015) Metabolome progression during early gut microbial colonization of gnotobiotic mice. Sci Rep 5:11589. doi: 10.1038/srep11589

Martin FPJ, Wang Y, Yap IKS, et al (2009) Topographical variation in murine intestinal metabolic profiles in relation to microbiome speciation and functional ecological activity. J Proteome Res 8:3464-3474. doi: 10.1021/pr900099x

Mellert W, Kapp M, Strauss V, et al (2011) Nutritional impact on the plasma metabolome of rats. Toxicol Lett 207:173-181. doi: 10.1016/j.toxlet.2011.08.013

Mingeot-Leclercq MP, Glupczynski Y, Tulkens PM (1999) Aminoglycosides: Activity and resistance. Antimicrob Agents Chemother 43:727-737. 
Modi SR, Collins JJ, Relman D a (2014) Antibiotics and the gut microbiota. J Clin Invest 124:4212-4218. doi: 10.1172/JCI72333.themselves

Musther H, Olivares-Morales A, Hatley OJD, et al (2014) Animal versus human oral drug bioavailability: Do they correlate? Eur J Pharm Sci 57:280-291. doi:

10.1016/j.ejps.2013.08.018

Nicholls AW, Mortishire-Smith RJ, Nicholson JK (2003) NMR Spectroscopic-Based Metabonomic Studies of Urinary Metabolite Variation in Acclimatizing Germ-Free Rats. Chem Res Toxicol 16:1395-1404. doi: 10.1021/tx0340293

Nicholson JK, Holmes E, Kinross J, et al (2012) Metabolic Interactions. 108:1262-1268.

Ottman N, Smidt H, de Vos WM, Belzer C (2012) The function of our microbiota: who is out there and what do they do? Front Cell Infect Microbiol 2:1-11. doi:

10.3389/fcimb.2012.00104

Paul E. Bendheim, Burkhard Poeggeler, Eyal Neria, Vivi Ziv MAP and DGC (2002)

Development of Indole-3-Propionic Acid ( OXIGON ${ }^{\mathrm{TM}}$ ) for Alzheimer's Disease.

J Mol Neurosci 19:213-217. doi: 10.1007/s12031-002-0036-0

Phipps a N, Stewart J, Wright B, Wilson ID (1998) Effect of diet on the urinary excretion of hippuric acid and other dietary-derived aromatics in rat. A complex interaction between diet, gut microflora and substrate specificity. Xenobiotica 28:527-537. doi: 10.1080/004982598239443

Poon K, Pang K (1995) Benzoic acid glycine conjugation in the isolated perfused rat kidney. Drug Metab Dispos 23:255-60.

Ramezani A, Massy Z a., Meijers B, et al (2015) Role of the Gut Microbiome in Uremia: A Potential Therapeutic Target. Am J Kidney Dis 1-16. doi:

10.1053/j.ajkd.2015.09.027

Ravenzwaay B Van, Kamp H, Montoya-parra GA, et al (2016) The development of a database for metabolomics - looking back on ten years of experience. 14:47-68.

Remer T, Manz F (2003) Paleolithic diet, sweet potato eaters, and potential renal acid load. Am. J. Clin. Nutr. 78:802-803; author reply 803.

Renshaw D, Cerniglia C, Mitsumori K NEOMYCIN (addendum, WHO Food Additives Series 51). http://www.inchem.org/documents/jecfa/jecmono/v51je02.htm. Accessed 11 Aug 2016

Roberts G (1995) Gentamicin (WHO Food Additives Series 34). In: WHO Food Addit. http://www.inchem.org/documents/jecfa/jecmono/v34je06.htm. Accessed 23 Aug 2016

Roessner U, Wagner C, Kopka J, et al (2000) Technical advance: simultaneous analysis of metabolites in potato tuber by gas chromatography-mass spectrometry. Plant $\mathrm{J}$ 23:131-42. doi: 10.1046/j.1365-313x.2000.00774.x

Saric J, Wang Y, Li J, et al (2008) Species variation in the fecal metabolome gives insight into differential gastrointestinal function. J Proteome Res 7:352-360. doi: $10.1021 / \mathrm{pr} 070340 \mathrm{k}$ 
Satoh M, Hayashi H, Watanabe M, et al (2003) Uremic Toxins Overload Accelerates Renal Damage in a Rat Model of Chronic Renal Failure. Nephron Exp Nephrol 95:e111e118. doi: 10.1159/000074327

Schoonen WGEJ, Kloks CP a M, Ploemen JPHTM, et al (2007) Uniform procedure of 1H NMR analysis of rat urine and toxicometabonomics Part II: Comparison of NMR profiles classification of hepatotoxicity. Toxicol Sci 98:286-297. doi:

10.1093/toxsci/kfm077

Scoper S V. (2008) Review of third-and fourth-generation fluoroquinolones in ophthalmology: In-vitro and in-vivo efficacy. Adv. Ther. 25:979-994.

Shah P, Swiatlo E (2008) A multifaceted role for polyamines in bacterial pathogens. Mol. Microbiol. 68:4-16.

Shiomi Y, Nishiumi S, Ooi M, et al (2011) GCMS-based metabolomic study in mice with colitis induced by dextran sulfate sodium. Inflamm Bowel Dis 17:2261-2274. doi: 10.1002/ibd.21616

Strahl N, Barr W (1971) Intestinal drug absorption and metabolism. 3. Glycine conjugation and accumulation of benzoic acid in rat intestinal tissue. J Pharm Sci 60:278-81.

Strauss V, Mellert W, Wiemer J, et al (2012) Increased toxicity when fibrates and statins are administered in combination - A metabolomics approach with rats. Toxicol Lett 211:187-200. doi: 10.1016/j.toxlet.2012.03.798

Strauss V, Wiemer J, Leibold E, et al (2009) Influence of strain and sex on the metabolic profile of rats in repeated dose toxicological studies. Toxicol Lett 191:88-95. doi: 10.1016/j.toxlet.2009.08.004

Swann JR, Want EJ, Geier FM, et al (2011) Systemic gut microbial modulation of bile acid metabolism in host tissue compartments. Proc Natl Acad Sci 108:4523-4530. doi: 10.1073/pnas.1006734107

Tabor CW, Tabor H (1985) Polyamines in microorganisms. Microbiol Rev 49:81-99.

Tanaka M, Takashina H, Tsutsumi S (2004) Comparative assessment of ocular tissue distribution of drug-related radioactivity after chronic oral administration of 14Clevofloxacin and 14C-chloroquine in pigmented rats. J Pharm Pharmacol 56:977-83. doi: 10.1211/0022357043932

Thomas S, Prabhu R, Balasubramanian KA (2005) Surgical manipulation of the intestine and distant organ damage - Protection by oral glutamine supplementation. Surgery 137:48-55. doi: 10.1016/j.surg.2004.04.038

Toh MC, Allen-Vercoe E (2015) The human gut microbiota with reference to autism spectrum disorder: considering the whole as more than a sum of its parts. Microb Ecol Health Dis 26:26309. doi: 10.3402/mehd.v26.26309

U.S. Food and Drug Administration Prescribing Information for DORYX Tablets. Reference ID: 2921043. http://www.accessdata.fda.gov/drugsatfda_docs/label/2011/050795s013lbl.pdf. Accessed 5 Jul 2016

U.S. Food and Drug Administration (2008) Levofloxacin - Prescribing Information. 
U.S. Food and Drug Administration (2013) FDA requires label changes to warn of risk for possibly permanent nerve damage from antibacterial fluoroquinolone drugs taken by mouth or by injection. http://www.fda.gov/Drugs/DrugSafety/ucm365050.htm.

Accessed 7 Jun 2016

U.S. Food and Drug Administration (2015) TETRACYCLINE HYDROCHLORIDE tetracycline hydrochloride capsule. In: Ref. ID 3740369.

http://www.accessdata.fda.gov/drugsatfda_docs/label/2015/050278s037lbl.pdf. Accessed 7 Jun 2016

Van Koten-Vermeulen JEM, van Leeuwen FXR (1995) Neomycin (WHO Food Additives Series 34). http://www.inchem.org/documents/jecfa/jecmono/v34je07.htm. Accessed 5 Jul 2016

Van Ravenzwaay B, Coelho-Palermo CG, Strauss V, et al (2010a) The use of metabolomics in cancer research. In: Cho WCS (ed) An Omics Perspective of Cancer. SpringerScience+Business Media B.V., New York, pp 141-166

Van Ravenzwaay B, Coelho-Palermo Cunha G, Strauss V, et al (2010b) The individual and combined metabolite profiles (metabolomics) of dibutylphthalate and di(2ethylhexyl)phthalate following a 28-day dietary exposure in rats. Toxicol Lett 198:159-170. doi: 10.1016/j.toxlet.2010.06.009

Van Ravenzwaay B, Cunha GCP, Leibold E, et al (2007) The use of metabolomics for the discovery of new biomarkers of effect. Toxicol Lett 172:21-28. doi: 10.1016/j.toxlet.2007.05.021

Van Ravenzwaay B, Herold M, Kamp H, et al (2012) Metabolomics: A tool for early detection of toxicological effects and an opportunity for biology based grouping of chemicals-From QSAR to QBAR. Mutat Res - Genet Toxicol Environ Mutagen 746:144-150. doi: 10.1016/j.mrgentox.2012.01.006

Vargas-Estrada D, Gutiérrez L, Juarez-Rodríguez I, Sumano H (2008) Pharmacokinetics of doxycycline and tissue concentrations of an experimental long-acting parenteral formulation of doxycycline in Wistar rats. Arzneimittelforschung 58:310-315. doi: 10.1055/s-0031-1296512.Pharmacokinetics

Velasquez-Manoff M (2015) 2. Gut Microbiome: The Peacekeepers. Nature 518:S3-S11. doi: $10.1038 / 518 \mathrm{~S} 3 \mathrm{a}$

Von Keutz E, Schlüter G (1999) Preclinical safety evaluation of moxifloxacin, a novel fluoroquinolone. J Antimicrob Chemother 43:91-100. doi: 10.1093/jac/43.suppl_2.91

Wikoff WR, Anfora AT, Liu J, et al (2009) Metabolomics analysis reveals large effects of gut microflora on mammalian blood metabolites. Proc Natl Acad Sci U S A 106:3698-3703. doi: 10.1073/pnas.0812874106

Williams RE, Eyton-Jones HW, Farnworth MJ, et al (2002) Effect of intestinal microflora on the urinary metabolic profile of rats: a $1 \mathrm{H}$-nuclear magnetic resonance spectroscopy study. Xenobiotica 32:783-794. doi: 10.1080/00498250210143047 
Wu IW, Hsu KH, Lee CC, et al (2011) P-cresyl sulphate and indoxyl sulphate predict progression of chronic kidney disease. Nephrol Dial Transplant 26:938-947. doi: 10.1093/ndt/gfq580

Yamada H, Yamahara A, Yasuda S, et al (2002) Dansyl chloride derivatization of methamphetamine: a method with advantages for screening and analysis of methamphetamine in urine. J Anal Toxicol 26:17-22. doi: 10.1093/jat/26.1.17

Zeng Y, Dai Z, Lu F, et al (2016) Emodin via colonic irrigation modulates gut microbiota and reduces uremic toxins in rats with chronic kidney disease. Oncotarget 7:1746817478. doi: 10.18632/oncotarget. 8160

Zhang Y-J, Li S, Gan R-Y, et al (2015) Impacts of Gut Bacteria on Human Health and Diseases. Int J Mol Sci 16:7493-7519. doi: 10.3390/ijms16047493

Zheng X, Xie G, Zhao A, et al (2011) The Footprints of Gut Microbial À Mammalian CoMetabolism. 10: 5512-5522. doi: 10.1021/pr2007945. 


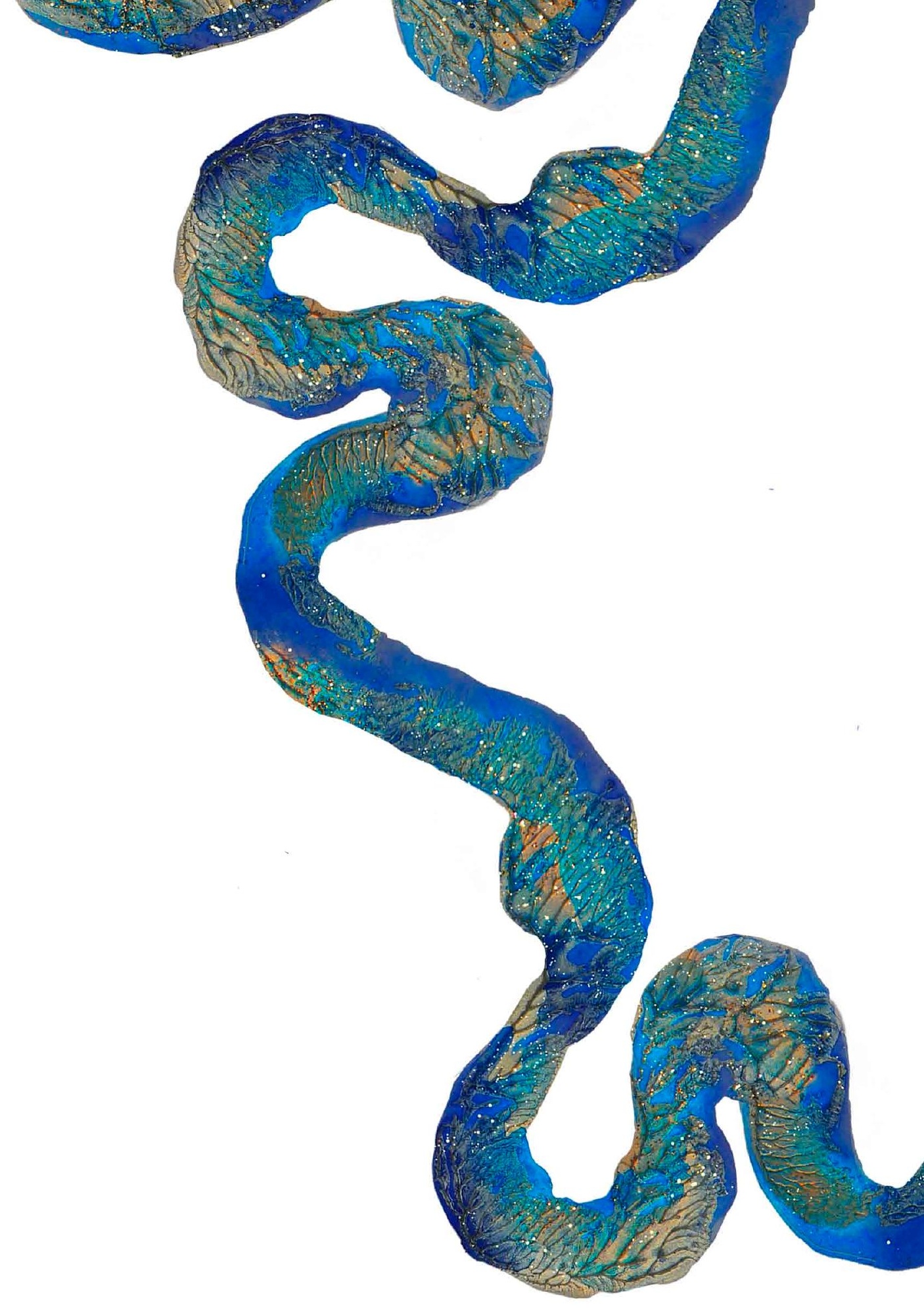




\section{Chapter 3}

Microbiome-related metabolite changes in gut tissue, cecum content and feces of rats treated with antibiotics

C. Behr, S. Sperber, X. Jiang, V. Strauss, H. Kamp, T. Walk, M. Herold K. Beekmann, I.M.C.M. Rietjens, B. van Ravenzwaay

Published in:

Toxicology and Applied Pharmacology 355 (2018) 198-210 


\section{AbSTRaCt}

The metabolic functionality of the gut microbiota contributes to the metabolism and wellbeing of its host, although detailed insight in the microbiota's metabolism is lacking. Omics technologies could facilitate unraveling metabolism by the gut microbiota.

In this study, we performed metabolite profiling of different matrices of the gut, after antibiotic treatment of rats in order to evaluate metabolite changes observed at different dose levels and in different sexes, and to identify the best tissue matrix for further investigations regarding an assessment of metabolic effects of new compounds with antibiotic activity. Three different antibiotics (vancomycin, streptomycin and roxithromycin) were administered orally to rats for 28 days according to the OECD 407 guideline with a subsequent metabolic profiling in feces, cecum content and gut tissue (jejunum, ileum, cecum, colon and rectum). The data were analyzed in the MetaMap ${ }^{\circledR}$ Tox database.

Treatment-related effects could be observed in the metabolite profile of feces and cecum content, but not of the different gut tissues. The metabolite profile showed compound specific effects on the microbiome. In line with the activity spectra of the antibiotics tested, vancomycin showed the largest effects, followed by roxithromycin and then by streptomycin for which changes were modest. In general, for all antibiotics the largest changes were observed for the classes of lipids (increase up to 94-fold), bile acids (increase up to 33 -fold), amino acids (increase up to 200-fold) and amino acid related (increase up to 348 -fold). The most relevant changes in metabolite values were similar in feces and cecum content and among sexes. The results of this targeted analysis indicate that the metabolic profiles of male and female animals in the gut microbiome are comparable. Concluding, taking other samples than feces does not add any extra information. Thus, as a non-invasive sampling method, feces provide a suitable matrix for studies on metabolism by the gut microbiota. 
Key words: metabolomics, metabolite profiling, gut content and tissue, gut microbiome, repeated dose oral toxicity study, antibiotics

$\begin{array}{ll}\text { ABBREVIATIONS } \\ \text { GC } & \text { gas chromatography } \\ \text { HD } & \text { high dose } \\ \text { IAA } & \text { indole-3-acetic acid } \\ \text { ILA } & \text { indole-3-lactic acid } \\ \text { LC } & \text { liquid chromatography } \\ \text { LD } & \text { low dose } \\ \text { MOA } & \text { mode of action } \\ \text { MS } & \text { mass spectrometry } \\ \text { OECD } & \text { Organization for Economic Cooperation and Development } \\ \text { PCA } & \text { principal component analysis } \\ \text { SPE } & \text { solid phase extraction } \\ \text { TAG } & \text { triacylglycerol }\end{array}$

\section{INTRODUCTION}

The intestinal microbiome plays an important role in health and disease of the host, and can be influenced by factors such as diet, the host condition, environmental chemical exposure and toxicological insult (e.g. antibiotics) (David et al., 2014; Modi et al., 2014; Rosenfeld, 2017; Zhang et al., 2015). The microbiome consists of trillions of microbial cells with a high inter- and intraspecies variability which makes it difficult to define a healthy gut microbiome regarding the species inhabiting the gut (Nguyen et al., 2015; Sender et al., 2016). However, the functional genes and the metabolic output of the microbes are likely to have a lower variability (Cho and Blaser, 2012; McCoy et al., 2017). Regarding this assumption, disease risks arising from gut microbial changes can be investigated by studying the microbiome's functionality, defined here as the production of metabolites. These metabolites can have a profound effect on the host, for example via nuclear receptor signaling, leading to changes in various pathways including for example energy metabolism and regulation of the immune system (Rooks and Garrett, 2016).

To investigate this complex relation of host and microbes, a better understanding of the crosstalk between host and gut microbes is essential. This can be done by measuring the molecules that enable this interaction, including especially metabolites formed by the microbiota available for uptake by the host. Metabolomics can serve as a powerful tool for assessing the functional status of the microbiome beyond taxonomics (Silbergeld, 2017; Wikoff et al., 2009). Other practical advantages of metabolomics include the possibility to use different matrices like urine, blood or feces, which also enables a longitudinal study design. Furthermore, with metabolomics a broad range of metabolites can be quantitatively measured and metabolic fluxes can be analyzed (Fernie et al., 2004). In the context of the 
complex mammalian-microbial co-metabolism, data obtained can be further used in a systems biology approach integrating these high-throughput data in computational network models (Heinken and Thiele, 2015).

In a previous study, we analyzed changes of the metabolome in plasma samples of male Wistar rats after administration of antibiotics known to induce an artificial shift of the intestinal microbiota (Behr et al., 2017). We measured small endogenous molecules such as carbohydrates, amino acids, nucleic acids or fatty acids and their derivatives resulting from biochemical pathways (Lindon et al., 2004). Using this approach, microbiome-related metabolites in the plasma could be identified and specific metabolite patterns could be established in the MetaMap ${ }^{\circledR}$ Tox database. This in vivo metabolomic database allows the prediction of systemic toxicity as well as the identification of substances with the same mode of action.

In the previous study it was difficult to distinguish between plasma metabolites derived from microbes and of endogenous origin. Therefore, in the current study we performed a metabolic profiling in different matrices of the gut after oral administration of broadspectrum antibiotics of different classes (see Figure 1). Vancomycin, streptomycin and roxithromycin were administered for 28 days in two dose levels to Wistar rats, and a targeted analysis of the same metabolites that were analyzed previously in plasma for usage in the MetaMap ${ }^{\circledR}$ Tox database were measured in feces, cecum content, and in tissues of jejunum, ileum, cecum, rectum and colon. Relative levels of metabolites in samples of treated rats were compared to the levels of these metabolites in untreated controls.

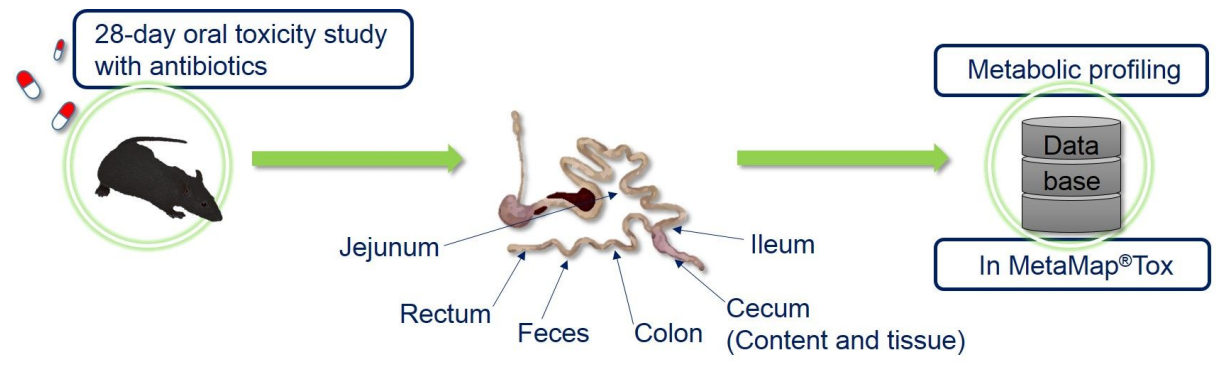

Fig. 1. Schematic presentation of the study design for metabolic profiling in different gut matrices of antibiotic treated rats. To identify metabolites related to microbiome changes due to antibiotic treatment, we have applied a metabolomics approach. Broad-spectrum antibiotics with each a different activity spectrum (vancomycin, streptomycin and roxithromycin) were administered orally to rats in a toxicity study. Feces, cecum content and gut tissue (jejunum, ileum, cecum, colon, rectum) were sampled for metabolic profiling on day 28 of the study.

The aim of this study was 1) to assess if the same metabolites previously detected in plasma could be found in the different gut tissues, 2) to investigate if a treatment-related effect can be observed in the analyzed tissues, and if different activity spectra of antibiotics are reflected in the metabolite profiles, 3) to evaluate if metabolite changes are observed at 
different dose levels and if they are sex-dependent, and 4) to identify the best sample matrix for the assessment of effects of new compounds affecting the gut microbiome.

\section{Materials ANd Methods}

Five independent studies were performed according to Organization for Economic Cooperation and Development (OECD) 407 guideline. Animal handling, treatment, and clinical examinations have been described earlier (Kamp et al., 2012; Strauss et al., 2012; van Ravenzwaay et al., 2012, 2010, 2007).

\section{Ethics statement}

The studies were approved by the BASF Animal Welfare Body, with the permission of the local authority, the Landesuntersuchungsamt Rheinland-Pfalz (approval number 23 17707/G 13-3-016). The study was performed in an AAALAC-approved (Association for Assessment and Accreditation of Laboratory Animal Care International) laboratory in accordance with the German Animal Welfare Act and the effective European Council Directive.

\section{Animals and Maintenance Conditions}

Briefly, male and female Wistar rats (CrI:WI(Han)) were supplied by Charles River, Germany, and were approximately $70 \pm 1$ days old at the beginning of the studies. The animals were maintained in an air-conditioned room at a temperature of 20 to $24^{\circ} \mathrm{C}$, a relative humidity of 30 to $70 \%$, and a 12 hour light / 12 hour dark cycle. Ground Kliba mouse/rat maintenance diet was supplied by Provimi Kliba SA, Kaiseraugst, Switzerland. Diet and drinking water were available ad libitum (except 16 - 20 hours before plasma sampling) and regularly assayed for chemical contaminants and the presence of microorganisms.

\section{Treatment of animals with compounds}

Treatment groups of five animals per group and sex were administered the test substance via gavage using an appropriate vehicle and metabolite changes were compared with a dietary control group (10 rats per sex). Dose levels of the antibiotics were selected in such a way that the low dose (LD) would show a low and reversible toxicological effect, while the high dose (HD) would show the toxicological mode of action. Dose-levels, routes of administration and form of preparation of the antibiotics are summarized in Table 1. 
Table 1 Compounds used, dose levels, form of preparation, and class of antibiotics. All compounds were administered orally by gavage.

\begin{tabular}{llll}
\hline Treatment & Dose $/$ day & Form of preparation & Class of antibiotics \\
\hline Vancomycin & 400 and $50 \mathrm{mg} / \mathrm{kg}$ body weight & in ultra-pure water & Glycopeptides \\
Streptomycin sulfate & 450 and $100 \mathrm{mg} / \mathrm{kg}$ body weight & $\begin{array}{l}\text { in water containing } \\
0.5 \% \mathrm{CMC}^{\mathrm{a}}\end{array}$ & Aminoglycosides \\
Roxithromycin & $600(400)^{\mathrm{b}}$ and $200 \mathrm{mg} / \mathrm{kg}$ body weight & $\begin{array}{l}\text { in water containing } \\
\text { in }\end{array}$ & Macrolides \\
\end{tabular}

${ }^{a}$ carboxymethyl cellulose: Tylose CB30000 ${ }^{\mathrm{b}}$ reduced on study day 3 in female and day 5 in male animals

\section{Sampling of gut tissues, cecum content and feces for metabolic profiling}

Gut tissue samples $(0.5-1 \mathrm{~cm})$, feces and cecum content were carefully removed during necropsy on day 28 after a fasting period of $16-20$ hours and one day after the last administration of the test substances. Besides feces and cecum content, the following gut tissues were taken for metabolite profiling: jejunum, ileum, rinsed cecum, colon and rectum. Cecum epithelium was rinsed with sterilized isotonic $\mathrm{NaCI}$ solution to remove the content. The samples were collected in pre-cooled (dry-ice) vials, immediately snap-frozen in liquid nitrogen and stored at $-80^{\circ} \mathrm{C}$ until metabolite profiling was performed.

\section{Clinical Examinations}

All animals were checked daily for any clinically abnormal signs and mortalities. Food consumption was determined on study days 6,13 and 27. Body weight was determined before the start of the administration period in order to randomize the animals and on study days 6,13 and 27. At the end of the treatment period, the animals were sacrificed by decapitation under isoflurane anesthesia. Metabolite profiling of the gut tissues, cecum content and feces samples was performed for all control and treated animals.

\section{Metabolite Profiling in gut tissues, cecum content and feces}

For mass spectrometry-based metabolite profiling, the gut tissue, feces and cecum content samples taken on study day 28 were extracted by a proprietary method as described below. Three types of mass spectrometry analysis were applied to all samples: GC-MS (gas chromatography-mass spectrometry) and LC-MS/MS (liquid chromatography-MS/MS) were used for broad profiling (van Ravenzwaay et al., 2007). Proteins were removed from $60 \mu \mathrm{L}$ plasma samples by precipitation using $200 \mu \mathrm{L}$ acetonitrile. Subsequently, polar and non-polar fractions were separated for both GC-MS and LC-MS/MS analysis by adding water and a mixture of ethanol and dichloromethane $(1: 2, \mathrm{v}: \mathrm{v})$. Feces and cecum content samples were freeze dried and ground prior to extraction. Feces, cecum content $(5 \mathrm{mg})$ and tissue samples $(10 \mathrm{mg})$ were extracted with a mixture of acetonitrile, water, ethanol and dichloromethane in a sample tube with a $3 \mathrm{~mm}$ stainless steel ball using a Bead Ruptor (Omni International Inc.). Phase separation was achieved by adding additional dichloromethane. For GC-MS analysis (CTC GC PAL, Agilent 6890 GC gas 
chromatograph, 5973 MSD mass spectrometer), the non-polar fraction was treated with methanol under acidic conditions to yield the fatty acid methyl esters derived from both free fatty acids and hydrolyzed complex lipids. The non-polar and polar fractions were further derivatized with O-methyl-hydroxylamine hydrochloride and pyridine to convert oxo-groups to O-methyl-oximes and subsequently with a silylating agent before analysis (Roessner et al., 2000). For LC-MS analysis, both fractions were reconstituted in appropriate solvent mixtures. HPLC was performed by gradient elution on reversed phase separation columns. Mass spectrometric detection technology was applied which allows target and high sensitivity MRM (Multiple Reaction Monitoring) profiling in parallel to a full screen analysis as described in patent WO2003073464 (Walk and Dostler, 2003). For GC-MS the acquisition in scan mode m/z 15-600 for polar compounds and m/z 40-600 for lipid compounds was applied. For LC-MS a MRM and Q3 Scan of m/z 100-1000 was used. MRMs off all analytes were determined using solutions of the authentic standard.

GC-MS conditions: CTC GC PAL, Agilent 6890 GC gas chromatograph, 5973 MSD mass spectrometer, gradient: $70{ }^{\circ} \mathrm{C}$ to $340{ }^{\circ} \mathrm{C}$, carrier gas: helium, acquisition in scan mode $\mathrm{m} / \mathrm{z}$ 15-600 (polar compounds) / m/z 40-600 (lipid compounds)

LC-MS conditions: Agilent 1100 HPLC System, AB Sciex API 4000 mass spectrometer, gradient elution for polar compounds with water / acetonitril / ammonium formate, gradient elution for lipid compounds with water / methanol / methyl tert-butyl ether / formic acid, MRM and Q3 Scan m/z 100-1000

For GC-MS and LC-MS/MS profiling, data were normalized to the median of reference samples which were derived from a pool formed from aliquots of all samples to account for inter- and intra-instrumental variation. The method resulted in 202 semi-quantitative analytes using the single peak signal of the respective metabolite and a normalization strategy according to the patent WO2007012643A1 (Walk et al., 2006) resulting in ratio values which represent the metabolite change of treated versus control animals, of which 175 are chemically identified and 27 are structurally unknown.

\section{Statistics}

The data were analyzed by univariate and multivariate statistical methods. The sex- and day-stratified heteroscedastic t-test ("Welch test") was applied to compare metabolite levels of dose groups with respective controls. The $\mathrm{p}$-values, $\mathrm{t}$-values, and ratios of corresponding group medians were collected as metabolic profiles and fed into the MetaMap®Tox database. Here, the profile strength of the metabolic profile is addressed. This parameter represents the rounded down average of absolute medians of t-values and does not only include the absolute number of significantly changed metabolites, but also the magnitude of the respective changes. For a detailed description on the use of statistics in MetaMap®Tox see van Ravenzwaay et al. (van Ravenzwaay et al., 2016). 
PCA scores plots and loadings of metabolites for all tissues and matrices on day 28 were generated in the statistical software $\mathrm{R}$ version 3.3.1. Since loadings represent the covariances/correlations between the original variables and the unit-scaled components, the values of each metabolite were used to establish a list of 25 metabolites with the highest loading for each PC1, 2 and 3 in the feces. Heatmaps of these metabolites after treatment with the three antibiotics were generated and compared to the corresponding values in cecum content (see Supplementary Material). In the heatmap data points statistically different $(\mathrm{p}<0.05)$ from control expressed as fold-change of control indicated within the heatmap square.

Whenever we write "significantly", "statistically significantly" is meant.

\section{Results}

\section{Clinical signs}

There were no mortalities in any of the treatment groups, except for one animal at the beginning of the study in the female low dose (LD) group of streptomycin. Animals which received the different antibiotics showed no signs of clinical toxicity, except for the high dose (HD) group of roxithromycin (slight salivation, piloerection, semiclosed eyelids, encrusted nose, general poor condition) and the corresponding LD group (slight salivation). Table 2 presents the food consumption and on body weight development for the different treatments.

Table 2 Relative changes in body weight and food consumption of male and female $\mathrm{Crl}$ :Wi(Han) rats $(\mathrm{N}=5$ per group) dosed for 28 days compared to the corre- sponding controls. Data were collected on study days 6, 13 and 27 , values in bold are statistically significantly decreased (Dunnett test (two-sided), $\mathrm{p}<0.05$ ) compared to controls (N=10 per group).

\begin{tabular}{|c|c|c|c|c|c|c|}
\hline \multirow{2}{*}{ Antibiotic } & \multirow{2}{*}{ Dose group } & \multirow{2}{*}{ Day } & \multicolumn{2}{|c|}{ Body weight } & \multicolumn{2}{|c|}{ Food consumption } \\
\hline & & & $\mathbf{m}$ & f & m & f \\
\hline \multirow{6}{*}{ Vancomycin } & \multirow{3}{*}{$\mathrm{HD}$} & 6 & 0.99 & 1.01 & 0.90 & 0.93 \\
\hline & & 13 & 0.98 & 1.02 & 0.96 & 0.93 \\
\hline & & 27 & 0.96 & 1.03 & 1.00 & 0.96 \\
\hline & \multirow{3}{*}{ LD } & 6 & 0.99 & 0.98 & 0.88 & 0.85 \\
\hline & & 13 & 0.97 & 1.00 & 0.93 & 0.92 \\
\hline & & 27 & 0.95 & 1.00 & 0.93 & 0.95 \\
\hline \multirow{6}{*}{ Streptomycin } & \multirow{3}{*}{$\mathrm{HD}$} & 6 & 0.99 & 1.01 & 0.92 & 0.97 \\
\hline & & 13 & 1.00 & 0.99 & 1.02 & 1.01 \\
\hline & & 27 & 1.00 & 1.00 & 0.98 & 0.96 \\
\hline & \multirow{3}{*}{ LD } & 6 & 0.97 & 0.98 & 0.92 & 0.91 \\
\hline & & 13 & 0.96 & 0.99 & 0.92 & 0.99 \\
\hline & & 27 & 0.95 & 0.97 & 0.97 & 0.93 \\
\hline \multirow{6}{*}{ Roxithromycin } & \multirow{3}{*}{$\mathrm{HD}$} & 6 & 0.88 & 0.90 & 0.36 & 0.43 \\
\hline & & 13 & 0.85 & 0.91 & 0.63 & 0.71 \\
\hline & & 27 & 0.88 & 0.94 & 0.94 & 0.85 \\
\hline & \multirow{3}{*}{ LD } & 6 & 0.98 & 0.99 & 0.85 & 0.80 \\
\hline & & 13 & 0.96 & 1.00 & 0.86 & 0.97 \\
\hline & & 27 & 0.96 & 1.01 & 1.04 & 1.03 \\
\hline
\end{tabular}


There was a reduction in food consumption in the animals receiving roxithromycin in the HD group on day 7 and 14 but not at day 28. Towards the end of the study (day 28), food consumption had returned to levels close to controls. Observed changes in body weight loss in this group were about $10 \%$ compared to the controls within the first days of treatment, showing that the treatment was above the MTD (maximum tolerated dose). For this reason, the dosage was reduced to $400 \mathrm{mg} / \mathrm{kg}$ bw/d from study day 3 in female animals study and from study day 5 in male animals.

\section{Metabolite Profiling}

Table 3 shows the percentage of changed metabolites of the metabolome analysis in feces and cecum content compared to control. The sampled gut matrices (jejunum, ileum, rinsed cecum, colon, rectum) did not show a change of the metabolome compared to the control (see PCAs in Supplementary Material).

In general, vancomycin had the most profound effect in the metabolome of feces and cecum content with $47-60 \%$ significantly altered metabolites out of 202 measured metabolites followed by roxithromycin (14-28\%) and streptomycin (8-20\%). The number of significantly upregulated metabolites was higher than the number of downregulated metabolites, except for the feces samples from the female animals of the LD and HD group of roxithromycin and the HD group of streptomycin. No dose-dependency could be observed based on the number of metabolite changes.

Table 3 Statistically significantly $(\mathrm{p}<0.05)$ downregulated (blue) and upregulated (red) measured metabolites compared to control in feces and cecum content of male and female Wistar rats after antibiotic treatment with vancomycin, roxithromycin and streptomycin for 28 days.

\begin{tabular}{|c|c|c|c|c|c|c|c|c|}
\hline & \multicolumn{4}{|c|}{ Vancomycin, males } & \multicolumn{4}{|c|}{ Vancomycin, females } \\
\hline & \multicolumn{2}{|c|}{ Feces } & \multicolumn{2}{|c|}{ Cecum content } & \multicolumn{2}{|c|}{ Feces } & \multicolumn{2}{|c|}{ Cecum content } \\
\hline & LD & HD & LD & HD & LD & HD & LD & HD \\
\hline Total metabolites & 194 & 195 & 188 & 191 & 190 & 193 & 192 & 193 \\
\hline$\%$ down & 10.8 & 5.6 & 14.4 & 11.5 & 15.3 & 13.5 & 16.1 & 15.5 \\
\hline$\%$ up & 45.9 & 41.5 & 42.0 & 48.7 & 40.5 & 34.7 & 36.5 & 35.2 \\
\hline \multirow[t]{4}{*}{$\%$ total } & 56.7 & 47.2 & 56.4 & 60.2 & 55.8 & 48.2 & 52.6 & 50.8 \\
\hline & \multicolumn{4}{|c|}{ Roxithromycin, males } & \multicolumn{4}{|c|}{ Roxithromycin, females } \\
\hline & \multicolumn{2}{|c|}{ Feces } & \multicolumn{2}{|c|}{ Cecum content } & \multicolumn{2}{|c|}{ Feces } & \multicolumn{2}{|c|}{ Cecum content } \\
\hline & LD & HD & LD & HD & LD & HD & LD & HD \\
\hline Total metabolites & 198 & 198 & 191 & 192 & 195 & 195 & 190 & 193 \\
\hline$\%$ down & 4.0 & 10.6 & 5.8 & 10.9 & 15.9 & 18.5 & 11.1 & 9.3 \\
\hline$\%$ up & 26.8 & 24.7 & 20.9 & 27.6 & 17.9 & 14.4 & 26.3 & 24.4 \\
\hline \multirow[t]{4}{*}{$\%$ total } & 30.8 & 35.4 & 26.7 & 38.5 & 33.8 & 32.8 & 37.4 & 33.7 \\
\hline & \multicolumn{4}{|c|}{ Streptomycin sulfate, males } & \multicolumn{4}{|c|}{ Streptomycin sulfate, females } \\
\hline & \multicolumn{2}{|c|}{ Feces } & \multicolumn{2}{|c|}{ Cecum content } & \multicolumn{2}{|c|}{ Feces } & \multicolumn{2}{|c|}{ Cecum content } \\
\hline & LD & HD & LD & HD & LD & HD & LD & HD \\
\hline Total metabolites & 195 & 197 & 191 & 189 & 193 & 189 & 187 & 190 \\
\hline$\%$ down & 4.1 & 1.5 & 6.3 & 3.2 & 5.2 & 14.8 & 4.3 & 8.9 \\
\hline$\%$ up & 13.8 & 18.8 & 13.1 & 19.6 & 8.3 & 16.9 & 8.0 & 14.2 \\
\hline$\%$ total & 17.9 & 20.3 & 19.4 & 22.8 & 13.5 & 31.7 & 12.3 & 23.2 \\
\hline
\end{tabular}


The number of significant changes between the feces and cecum content of males and females were in the same range, except the female group of the roxithromycin treatment. Here, the number of significantly upregulated metabolites in the cecum content was about $10 \%$ higher than in the feces, while the number of downregulated metabolites was on average about $7 \%$ higher in feces than in cecum content.

In the MetaMap ${ }^{\circledR}$ Tox database the profile strengths of the metabolic profiles were calculated (see Table 4). This parameter includes the magnitude of the changes. In the database, the profile strength ranges between 0.49 and 5.58, with an average over all treatments of 1.43. For one, it clearly reflects the impact of each antibiotic on the metabolome, with vancomycin having the most profound effect, followed by roxithromycin and streptomycin. For another, the values also show the similarity between males and females and between both matrices.

Table 4 Profile strength of the metabolite profile in feces and cecum content of male and female Wistar rats after antibiotic treatment after treatment with vancomycin, roxithromycin and streptomycin.

\begin{tabular}{|c|c|c|c|c|c|c|c|c|c|c|c|c|}
\hline & \multicolumn{3}{|c|}{ Vancomycin } & \multicolumn{4}{|c|}{ Roxithromycin } & \multicolumn{4}{|c|}{ Streptomycin } & \multirow[b]{2}{*}{$\mathrm{m}, \mathrm{HD}$} \\
\hline & f, LD & m, LD & f, HD & $m, H D$ & f, LD & $m$, LD & $f, H D$ & $\mathrm{~m}, \mathrm{HD}$ & f, LD & m, LD & $f, H D$ & \\
\hline & 5.08 & 5.26 & 4.35 & 5.16 & 2.13 & 2.13 & 2.17 & 2.37 & 1.48 & 1.63 & 1.81 & 1.85 \\
\hline Feces & 4.87 & 5.48 & 3.86 & 4.19 & 2.17 & 2.19 & 2.13 & 2.31 & 1.46 & 1.69 & 2.22 & 1.99 \\
\hline
\end{tabular}

\section{Treatment-related effect on feces and cecum content}

To reveal treatment-related effects on the metabolites in the matrices analyzed, a principal component analysis (PCA) was performed. In feces and cecum content, a clear treatmentrelated effect could be observed. However, there were no differences in the metabolites in gut tissue samples (i.e. jejunum, ileum, rinsed cecum, colon, rectum) between treatment and control. The corresponding PCAs of the gut tissues can be found in the supplementary material.

Feces and cecum content from males and females showed a similar pattern: vancomycin and roxithromycin showed a clear separation from each other and from the control group. For the feces samples, the male group treated with streptomycin is separated from the control and also from the vancomycin and roxithromycin data points, while in the cecum samples separation from streptomycin from the control was less evident (see PCAs in Figure 2, Figure 3, and Figure 4). In female animals, the streptomycin data were not separated from the control group in both feces and cecum content. Vancomycin showed a separation between LD and HD over all groups for both matrices and gender. This could also be observed for streptomycin in the feces samples of male animals and in the principal component (PC) 3 for both matrices (see Figure 4).

\section{Top25 metabolites of feces compared to cecum content}

To identify the metabolites that drive the separation of the different antibiotic treatments from the control, the loadings of the first three principal components for each metabolite, 
matrix and sex were extracted. A ranking of the 25 metabolites with the highest loadings in the feces against the corresponding loadings of metabolites of the cecum content was done and heatmaps with the analyzed metabolite values from day 28 of male and female animals were established (see supplementary material). For each treatment, the focus was set on the principal component which shows the clearest separation from the control in the PCA. Therefore, for vancomycin loadings of the PC1, for roxithromycin loadings of the PC2 and for streptomycin loadings of the PC3 were evaluated (see Figure 2, Figure 3, and Figure 4).

\section{Vancomycin}

Compared to roxithromycin and streptomycin, vancomycin had the most profound effect on the metabolites in feces and cecum content. The 25 metabolites with the highest loadings in feces of vancomycin treated animals were all upregulated for both males and females in HD and LD, with creatinine and trans-4-hydroxyproline being upregulated more than 100-fold (see Figure 2).

In the male group, triacylglycerols (TAGs) and other lipids, the amino acids valine, proline, leucine, histidine, alanine, isoleucine, glycine and phenylalanine were highly upregulated, as well as other metabolites including creatine, 2-hydroxybutyrate and pantothenic acid. Cholic acid was increased in all samples except in cecum content of the HD vancomycin group. Furthermore, two TAGs, pantothenic acid and 4-hydroxysphinganine were found only in the top 25 of feces but not in that of cecum content. In cecum content of the male group 3-hydroxybutyrate, lysine and lactate were significantly upregulated.

The female group showed a similar pattern of changes with a significant upregulation of TAGs, valine, leucine, proline, phenylalanine, isoleucine, alanine, histidine and glycine as well as creatine, 2- and 3-hydroxybutyrate, pantothenic acid, three unknown metabolites and a cholesterylester. Similar to the male group, cholic acid was upregulated in the feces but downregulated in the cecum content of the HD group. Besides cholic acid, two unknown metabolites and a cholesterylester just showed up in the top25 of the feces. In cecum content, lactate, myo-inositol-2-phosphate and lysophosphatidylcholine (C18:1) were significantly upregulated instead. 


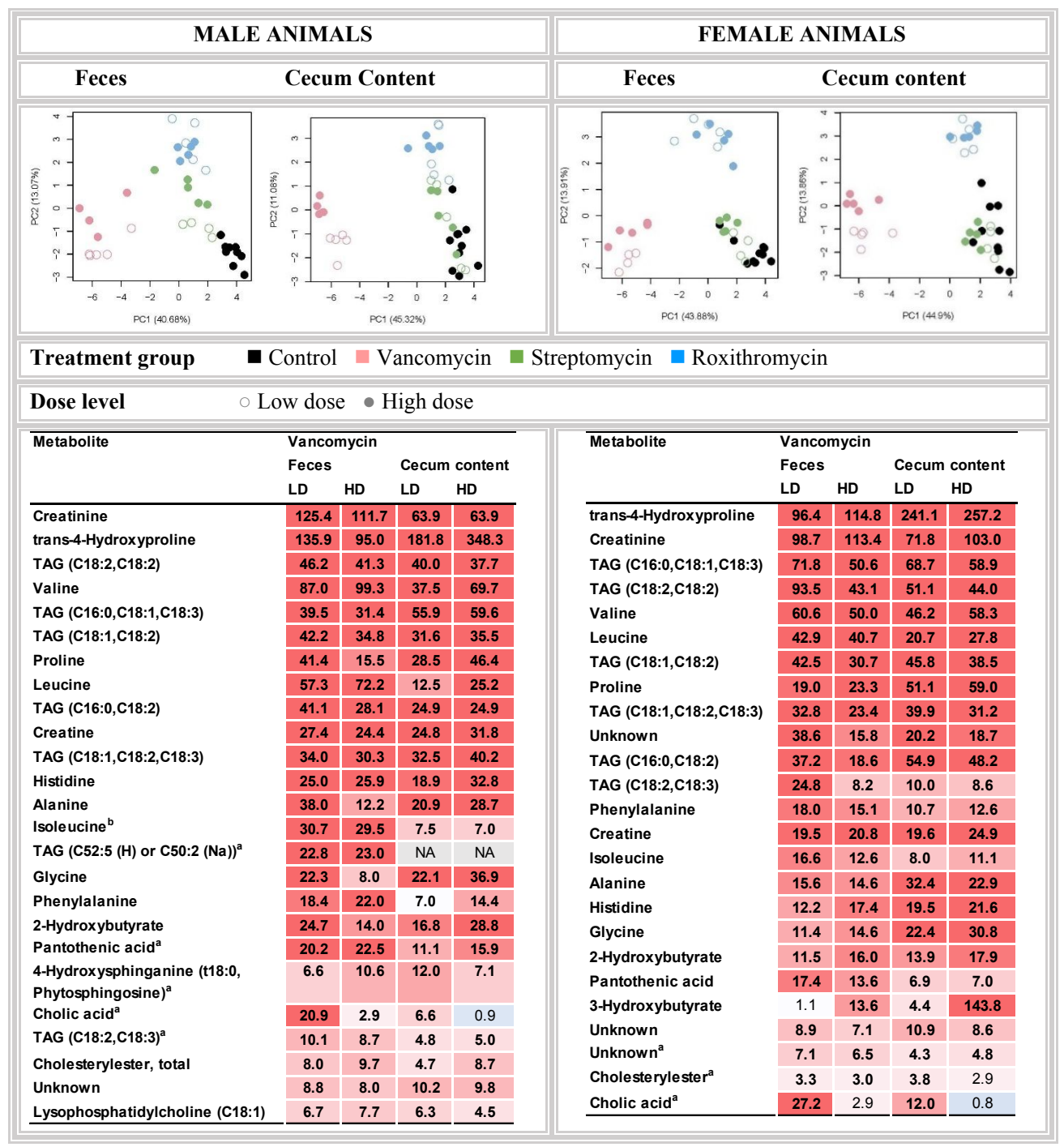

Fig. 2. PCAs of tested antibiotics and heatmap of top 25 metabolites of feces for vancomycin. Metabolite concentration changes in feces and cecum content of male $(\mathrm{m})$ and female (f) $\mathrm{Crl}$ :Wi(Han) rats (N=5 per group) dosed with vancomycin (50 and $400 \mathrm{mg} / \mathrm{kg}$ bw/d) for 28 days. Statistically significant changes (Welch-t-test; $\mathrm{p}<$ 0.05 ) are shown in bold, red boxes mean upregulation and blue boxes downregulation. The intensity of the color corresponds to the magnitude of the fold change. "NA" indicates that for this analyte, treatment and/or analysis groups there are no contrasts available. a just in top25 of feces; in cecum content, males: 3 - hydroxybutyrate, lysine, lactate; in cecum content, females: lactate, myo-inositol-2-phosphate, lysophosphatidylcholine (C18:1). b measured with LC-MS/MS and LC- MS. 


\section{Roxithromycin}

Roxithromycin had a lower impact on the metabolome than vancomycin based on the number and fold change of altered metabolites. Highest upregulation in both matrices of males and females was observed for allantoin, taurine, taurocholic acid, creatinine, kynurenic acid and isoleucine (see Figure 3). Malate and the amino acids tryptophan, tyrosine, asparagine, threonine, methionine and arginine were increased in all groups as well.

In the male group, indole-3-lactic acid (ILA), lysophosphatidylcholine, myristic acid, glucose-6-phosphate, two unknown metabolites and aspartate was increased in feces samples but were not among the top 25 metabolites of cecum content. Here, significantly altered were glucose, ornithine, leucine, glutamine, serine, phenylalanine, phosphatidylcholine (C18:0, C20:4).

Contrary to the males, in the female group indole-3-acetic acid (IAA) was downregulated in feces and cecum content but only significantly in the cecum content LD. ILA was slightly increased in the feces. Phosphate, kynurenic acid, ketoleucine, IAA, lysophophatidylcholine, phenylalanine and an unknown metabolite just showed up in the top25 of the feces. A higher impact in cecum content was observed for the metabolites serine, glutamine, ornithine, hexoses, aspartate, lysine.

\section{Streptomycin}

Treatment with streptomycin showed a separation from the control in a plot of PC 1 versus PC 3 (see Figure 4). In both matrices, the HD group showed a separation from the control and a slight separation of the LD group. The overall effect on the feces metabolome after streptomycin treatment was less pronounced in female compared to male animals. In the top25 metabolite patterns of the feces, the treatment with streptomycin showed clear differences in quantitative changes and significantly altered values. Similarities in both matrices of males and females were observed for kynurenic acid and cysteine which were significantly upregulated. An increase of the amino acids taurine, valine, isoleucine and leucine was observed in the HD, for leucine only in the feces.

In the male group, pantothenic acid was significantly upregulated in cecum content and feces. Interestingly, the LD group showed a 3-5-fold higher upregulation compared to the HD group. Also increased, though not consistently over all dose groups, were biotin, succinate, uracil, ILA, threonine, glucosamine, malate, glycerol and glucose. Glycochenodeoxycholic acid was significantly decreased in the HD group of cecum content and ethanolamine plasmalogen was significantly decreased in the LD group for both matrices. Both bile acids, ILA, salicylic acid, glucosamine, an unknown metabolite, leucine, glycerol, ethanolamine and elaidic acid were found in the top25 of feces samples but were not present in the top25 of cecum content. In cecum content calcitriol, lysophosphatidylcholine (C17:0), threo-sphingosine (d18:1), 5-O-methylsphingosine (d18:1), four unknown metabolites, 3,3',5-triiodothyronine, and significantly altered spermidine were found (data not shown). 
In the feces of the female group taurocholic and cholic acid were both significantly upregulated. In both matrices an upregulation of spermidine, TAG and glycerol-3phosphate was observed.

Sphingomyelin, isoleucine, three bile acids, leucine, glucosamine just showed up significantly upregulated in the top 25 of the feces. In cecum content the following metabolites were significantly altered in the top25: three TAGs ((C16:0,C18:2); (C18:1,C18:2,C18:3) (C18:2,C18:2), (C18:1,C18:2)), erythro-sphingosine, docosapentaenoic acid (C22: $\operatorname{cis}[7,10,13,16,19] 5)$ and uric acid. 


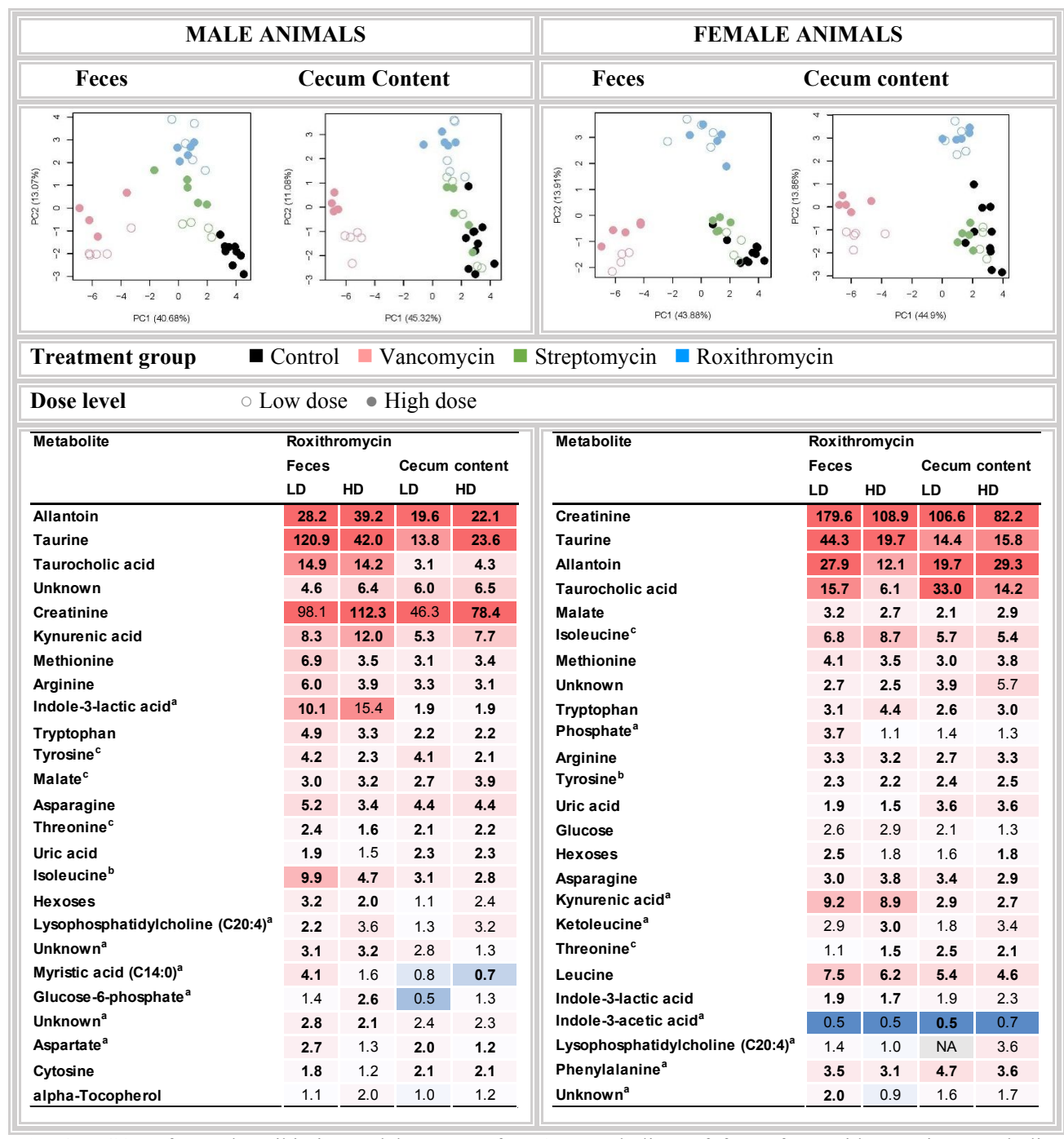

Fig. 3. PCAs of tested antibiotics and heatmap of top 25 metabolites of feces for roxithromycin. Metabolite concentration changes in feces and cecum content of male $(\mathrm{m})$ and female (f) $\mathrm{Crl}$ :Wi(Han) rats (N=5 per group) dosed with roxithromycin (200 and $400 \mathrm{mg} / \mathrm{kg}$ bw/d) for 28 days. Statistically significant changes (Welch-t- test; $\mathrm{p}$ $<.05)$ are shown in bold, red boxes mean upregulation and blue boxes downregulation. The intensity of the color corresponds to the magnitude of the fold change. "NA" indicates that for this analyte, treatment and/or analysis groups there are no contrasts available. a just in top25 of feces; in cecum content, males: glucose, ornithine, leucine, glutamine, serine, phenylalanine, phosphatidylcholine (C18:0,C20:4), lysine; in cecum content, females: serine, glutamine, ornithine, hexoses, aspartate, lysine, an unknown metabolite. b measured with LC-MS/MS and LC-MS. c measured with GC-MS. 


\begin{tabular}{|c|c|c|c|c|c|c|c|c|c|}
\hline \multicolumn{5}{|c|}{ MALE ANIMALS } & \multicolumn{5}{|c|}{ FEMALE ANIMALS } \\
\hline Feces & \multicolumn{4}{|c|}{ Cecum Content } & \multicolumn{5}{|c|}{ Cecum content } \\
\hline 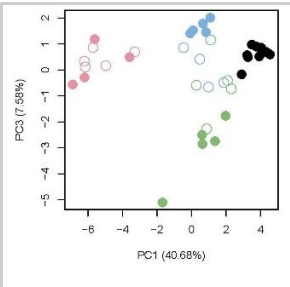 & & $\begin{array}{ll}1 & 1 \\
-6 & -4\end{array}$ & . & $\begin{array}{l}\vdots \\
\dot{3} \cdot \\
\dot{y} \cdot\end{array}$ & 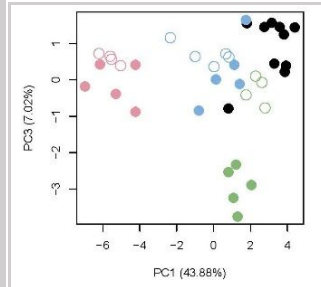 & 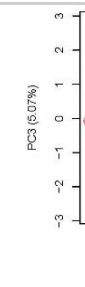 & \multicolumn{3}{|c|}{ 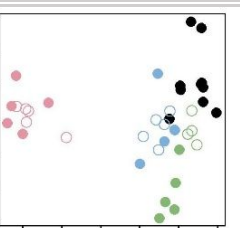 } \\
\hline \multicolumn{5}{|l|}{ Treatment group } & \multicolumn{5}{|c|}{ Streptomycin $\square$ Roxithromycin } \\
\hline Dose level & ow dos & se $\bullet$ & ligh d & & & & & & \\
\hline \multirow[t]{3}{*}{ Metabolite } & \multicolumn{4}{|c|}{ Streptomycin sulfate } & Metabolite & \multicolumn{4}{|c|}{ Streptomycin sulfate } \\
\hline & \multicolumn{2}{|c|}{ Feces } & \multicolumn{2}{|c|}{ Cecum content } & & \multicolumn{2}{|c|}{ Feces } & \multicolumn{2}{|c|}{ Cecum content } \\
\hline & & HD & LD & & & LD & HD & LD & HD \\
\hline Pantothenic acid & 102.1 & 19.8 & 61.8 & 20.2 & Taurine & 0.8 & 32.2 & 0.5 & 19.4 \\
\hline Cholic acid $^{\mathrm{a}}$ & 2.0 & 3.3 & 1.3 & 0.5 & Kynurenic acid & 23.5 & 24.0 & 6.8 & 6.9 \\
\hline Biotin & 1.3 & 8.7 & 3.7 & 1.9 & Valine & 1.1 & 8.3 & 0.8 & 11.6 \\
\hline Valine & 8.0 & 32.8 & 1.9 & 13.0 & Sphingomyelin (d18:1,C16:0) ${ }^{\mathrm{a}}$ & 2.5 & 8.7 & NA & NA \\
\hline Taurine & 8.2 & 199.5 & 9.7 & 42.6 & Cysteine & 3.8 & 8.4 & 2.2 & 5.2 \\
\hline Isoleucine $^{c}$ & 6.3 & 15.2 & 2.0 & 6.6 & Isoleucine $^{a, b}$ & 1.3 & 2.8 & 0.8 & 4.5 \\
\hline Succinate & 1.3 & 19.7 & 1.4 & 6.4 & Taurocholic acid $^{\mathrm{a}}$ & 4.5 & 4.5 & 3.8 & 2.3 \\
\hline Kynurenic acid & 32.2 & 21.0 & 15.7 & 14.1 & Cholic acid $^{\mathrm{a}}$ & 7.7 & 2.9 & 2.9 & 0.8 \\
\hline $\begin{array}{l}\text { Uracil } \\
\text { Indole-3-lactic acid }\end{array}$ & $\begin{array}{l}21.7 \\
30.7\end{array}$ & $\begin{array}{c}2.1 \\
25.6\end{array}$ & $\begin{array}{l}17.0 \\
1.7\end{array}$ & $\begin{array}{l}2.6 \\
2.0\end{array}$ & Spermidine & 3.8 & 4.7 & 2.1 & 3.2 \\
\hline $\begin{array}{l}\text { Choline plasmalogen (C18- } \\
\text { vinyl C20:4) }\end{array}$ & 1.5 & 2.4 & 1.3 & 3.2 & $\begin{array}{l}\text { dihomo-gamma-Linolenic acid } \\
\text { (C20:cis[8,11,14]3) }\end{array}$ & 1.2 & 2.9 & 1.6 & 4.7 \\
\hline $\begin{array}{l}\text { vinyl,C20:4) } \\
\text { Threonine }^{\mathrm{b}}\end{array}$ & 08 & & & & Leucine $^{a}$ & 1.5 & 4.5 & 0.8 & 3.0 \\
\hline Salicylic acid ${ }^{a}$ & 2.2 & $\begin{array}{l}9.9 \\
1.3\end{array}$ & $\begin{array}{l}1.0 \\
0.9\end{array}$ & 0.6 & Unknown & 1.8 & 3.6 & 1.5 & 2.1 \\
\hline Glucosamine $^{a}$ & 1.7 & 4.1 & 1.3 & 2.1 & Glycerol & 1.1 & 2.3 & 2.3 & 4.7 \\
\hline Unknown ${ }^{\mathrm{a}}$ & 1.6 & 3.2 & 2.3 & 4.1 & Glucosamine $^{\mathrm{a}}$ & 1.7 & 2.6 & 0.8 & 1.0 \\
\hline Cysteine & 4.0 & 9.6 & 2.4 & 5.5 & Glycerol-3-phosphate & 1.6 & 2.3 & 1.9 & 3.3 \\
\hline Malate $^{\mathrm{b}}$ & 0.9 & 7.3 & 1.2 & 20.4 & Sphingomyelin (d18:2,C18:0) & 0.6 & 1.7 & 0.9 & 2.8 \\
\hline Leucine $^{a}$ & 3.5 & 12.7 & 1.6 & 3.2 & Pantothenic acid & 1.3 & 2.2 & 0.8 & 2.8 \\
\hline Glycochenodeoxycholic acid ${ }^{a}$ & 0.8 & 0.5 & 0.4 & 0.2 & Tyrosine $^{a, b}$ & 1.1 & 1.1 & 0.8 & 2.2 \\
\hline Glycerol $^{\mathrm{a}}$ & 4.7 & 1.8 & 1.5 & 1.3 & Uracil $^{\mathrm{a}}$ & 1.2 & 1.1 & 0.7 & 1.0 \\
\hline erythro-Sphingosine (d18:1) & 1.1 & 2.4 & 2.0 & 3.2 & TAG (C16:0,C18:1,C18:3) & 1.8 & 5.4 & 3.2 & 4.5 \\
\hline Ethanolamine plasmalogen & 0.6 & 2.5 & 0.5 & 1.6 & DAG (C18:1,C18:2) & 1.1 & 2.7 & 1.1 & 1.8 \\
\hline$(C 39: 5)^{\mathrm{a}}$ & & & & & Unknown $^{\mathrm{a}}$ & 1.6 & 1.8 & 1.0 & 1.1 \\
\hline Elaidic acid (C18:trans[9]1) ${ }^{\mathrm{a}}$ & NA & 1.7 & NA & 8.1 & 4-Hydroxyphenylpyruvate ${ }^{a}$ & 0.8 & 0.9 & 0.9 & 1.3 \\
\hline Phosphate & 2.8 & 0.7 & 2.6 & 1.8 & Glycochenodeoxycholic acid & 0.7 & 0.8 & 0.9 & 0.6 \\
\hline Glucose & 1.0 & 4.0 & 0.5 & 0.7 & Unknown & 1.3 & 3.5 & 1.6 & 3.0 \\
\hline
\end{tabular}

Fig. 4. PCAs of tested antibiotics and heatmap of top 25 metabolites of feces for streptomycin. Metabolite concentration changes in feces and cecum content of male $(\mathrm{m})$ and female (f) $\mathrm{Crl}$ :Wi(Han) rats (N=5 per group) dosed with Streptromycin (100 and $450 \mathrm{mg} / \mathrm{kg}$ bw/d) for 28 days. Statistically significant changes (Welch-t- test; $\mathrm{p}$ $<0.05)$ are shown in bold, red boxes mean upregulation and blue boxes downregulation. The intensity of the color corresponds to the magnitude of the fold change. "NA" indicates that for this analyte, treatment and/or analysis groups there are no contrasts available. ${ }^{a}$ just in top 25 of feces; statistically significantly in cecum content, males: spermidine, an unknown metabolite; in cecum content, females: TAG (C16:0,C18:2), erythro-sphingosine (d18:1), TAG (C18:1,C18:2,C18:3), TAG (C18:2,C18:2), TAG (C18:1,C18:2), docosapentaenoic acid (C22: $\operatorname{cis}[7,10,13,16,19] 5)$, uric acid. ${ }^{\mathrm{b}}$ measured with LC-MS/MS and LC-MS. ${ }^{\mathrm{c}}$ measured with GC-MS. 


\section{Overall comparison of statistically significantly metabolite changes}

The analysis of the top 25 metabolites showed the most important metabolites that drive the separation of the treatments in the PCA. Independent from the PCA, a separate analysis was performed to compare similar metabolite changes as well as only downregulated metabolites in the different dose groups and target tissues.

The metabolite profiles of the feces after antibiotic treatment for both sexes were screened for overlapping metabolites and compared to metabolite changes in cecum content (see Table 5). Eight metabolites in the male groups and seven metabolites in the female group were found to be significantly changed in all dose groups. Two metabolites were significantly changed in both sexes: kynurenic acid, which was increased, and stearic acid, which was decreased compared to controls. In the male group, all other metabolites were upregulated (lipids, amino acids and citrate in feces) whereas in the female group three more metabolites were downregulated (lipids). In all female groups, beta-sitosterol as well as taurocholic acid were upregulated. Only in the LD group of vancomycin, taurocholic acid was downregulated.

Further, the decreased metabolites in feces for both sexes were evaluated and compared. Table 6 shows statistically significantly decreased metabolites in feces after the treatment with each of the antibiotics for males and females of LD and HD. Vancomycin treatment led to the highest number of significant changes, closely followed by roxithromycin and then streptomycin. In general, female animals showed a higher number of significantly decreased metabolites. The majority of these belong to the class of complex lipids, fatty acids and related compounds. After vancomycin treatment, feces of both sexes showed a decrease of glutamate, 5-oxoproline, IAA, 18-hydroxy-11-deoxycorticosterone, alphatocopherol and coenzyme Q9 and Q10. 18-hydroxy-11-deoxycorticosterone was also decreased after roxithromycin treatment as well as coenzyme 9. In vancomycin treated males 4-hydroxyphenylpyruvate was decreased, in females salicylic acid, heptadecanoic acid (C17:0) and an unknown metabolite were decreased. In males of roxithromycin treatment IAA was decreased, and in females myo-inositol, myristic acid (C14:0), behenic acid (C22:0), erythro-sphingosine (d18:1), ceramide (d18:1, C24:0), 3-hydroxybutyrate, coenzyme Q10 and alpha-tocopherol. In females but not in males, octadecanol, lignoceric acid (C24:0), heptadecanoic acid (C17:0), eicosanoic acid (C20:0) and behenic acid (C22:0) were decreased after streptomycin treatment. 
Table 5 Overall comparison of metabolite changes after antibiotic treatment found in the metabolite profiles of feces compared to cecum content. Metabolite concentration changes in feces of male and female Crl:Wi(Han) rats ( $\mathrm{N}=5$ per group) dosed with vancomycin ( 50 and $400 \mathrm{mg} / \mathrm{kg} \mathrm{bw/d}$ ), roxithromycin (200 and 400 (600) mg/kg bw/d) and streptomycin (100 and $450 \mathrm{mg} / \mathrm{kg}$ bw/d) for 28 days. Statistically significant changes (Welch-t-test; $\mathrm{p}<.05)$ are shown in bold, blue boxes mean downregulation, red boxes upregulation. The intensity of the color cor- responds to the magnitude of the fold change.

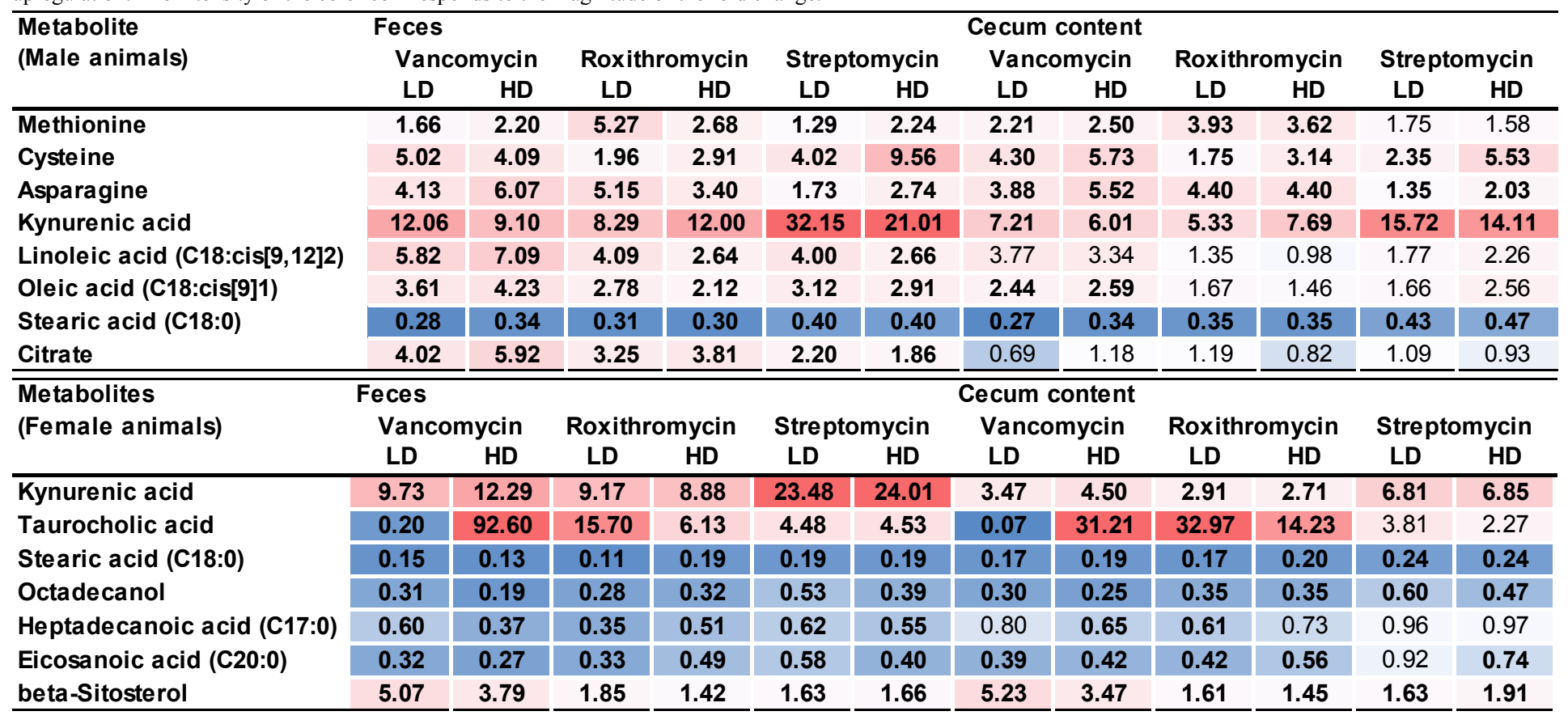


Microbiome-related metabolite changes in the intestinal tract

Table 6 Heatmap of statistically significantly decreased values of metabolite changes in feces after treatment with antibiotics. Metabolite concentration changes in feces of male (m) and female (f) Crl:Wi(Han) rats (N=5 per group) dosed with vancomycin (50 and $400 \mathrm{mg} / \mathrm{kg}$ bw/d), roxithromycin (200 and 400 (600) mg/kg bw/d) and streptomycin (100 and $450 \mathrm{mg} / \mathrm{kg} \mathrm{bw} / \mathrm{d}$ ) for 28 days. Statistically significant changes (Welch-t-test; $\mathrm{p}<.05$ ) are shown in bold, blue boxes mean down- regulation. The intensity of the blue color corresponds to the magnitude of the fold change.

\begin{tabular}{|c|c|c|c|c|c|c|c|c|c|c|c|c|c|}
\hline \multirow[t]{2}{*}{ Metabolite } & \multicolumn{2}{|c|}{ Vancomycin } & \multicolumn{6}{|c|}{ Roxithromycin } & \multicolumn{2}{|c|}{ Streptomycin } & \multirow[b]{2}{*}{ f, HD } & \multirow[b]{2}{*}{ m, HD } & \multirow{2}{*}{ Metabolite class } \\
\hline & f, LD & m, LD & f, HD & m, HD & f, LD & m, LD & f, HD & m, HD & f, LD & m, LD & & & \\
\hline Glutamate & 0.20 & 0.16 & 0.11 & 0.24 & & & & & & & & & Amino acids \\
\hline 4-Hydroxyphenylpyruvate & 0.56 & 0.30 & 0.56 & 0.40 & & & & & & & & & \\
\hline 5-Oxoproline & 0.48 & 0.35 & 0.35 & 0.43 & & & & & & & & & Amino acids related \\
\hline Indole-3-acetic acid & 0.12 & 0.18 & 0.10 & 0.08 & 0.54 & 0.11 & 0.50 & 0.26 & & & & & \\
\hline myo-Inositol & & & & & 0.20 & 1.20 & 0.30 & 1.58 & & & & & Carbohydrates and related \\
\hline 17-Methyloctadecanoic acid & & & & & 0.41 & 0.70 & 0.58 & 0.70 & & & & & Complex lipids, fatty acids and related \\
\hline Behenic acid (C22:0) & & & & & 0.60 & 0.82 & 0.50 & 1.01 & 0.61 & 1.26 & 0.47 & 0.81 & \\
\hline Ceramide (d18:1, C24:0) & & & & & 0.71 & 0.83 & 0.70 & 0.62 & & & & & \\
\hline Cholic acid & & & & & 0.29 & 0.42 & 0.46 & 0.22 & & & & & \\
\hline Eicosaenoic acid (C20:1) No 02 & 0.48 & 0.58 & 0.34 & 0.46 & & & & & & & & & \\
\hline Eicosanoic acid (C20:0) & 0.32 & 0.31 & 0.27 & 0.39 & 0.33 & 0.64 & 0.49 & 0.36 & 0.40 & 0.45 & 0.58 & 0.79 & \\
\hline erythro-Sphingosine (d18:1) & & & & & 0.39 & 0.54 & 0.60 & 0.74 & & & & & \\
\hline Glycerol-3-phosphate, polar fraction & 0.39 & 0.33 & 0.27 & 0.38 & & & & & & & & & \\
\hline Heptadecanoic acid (C17:0) & 0.37 & 1.02 & 0.60 & 0.98 & 0.35 & 0.79 & 0.51 & 0.79 & 0.62 & 1.44 & 0.55 & 1.07 & \\
\hline Hexadecanol & 0.29 & 0.13 & 0.21 & 0.10 & 0.33 & 0.43 & 0.46 & 0.32 & & & & & \\
\hline Isopalmitic acid (C16:0) & 0.34 & 0.45 & 0.25 & 0.50 & & & & & & & & & \\
\hline Lignoceric acid (C24:0) & & & & & 0.44 & 0.71 & 0.53 & 0.60 & 0.58 & 1.07 & 0.59 & 0.79 & \\
\hline Myristic acid (C14:0) & & & & & 0.33 & 1.64 & 0.24 & 4.05 & & & & & \\
\hline Octadecanol & 0.31 & 0.20 & 0.19 & 0.16 & 0.28 & 0.48 & 0.32 & 0.30 & 0.53 & 0.65 & 0.39 & 0.80 & \\
\hline Palmitic acid (C16:0) & 0.61 & 0.68 & 0.50 & 0.77 & 0.42 & 1.01 & 0.73 & 0.58 & & & & & \\
\hline Sphingomyelin (d18:2,C18:0) & 0.51 & 0.45 & 0.54 & 0.48 & 0.56 & 0.65 & 0.62 & 0.63 & & & & & \\
\hline Stearic acid (C18:0) & 0.15 & 0.28 & 0.13 & 0.34 & & & & & 0.19 & 0.40 & 0.19 & 0.40 & \\
\hline Tricosanoic acid (C23:0) & 0.54 & 0.56 & 0.42 & 0.91 & & & & & & & & & \\
\hline Unknown lipid & 0.41 & 0.89 & 0.42 & 1.41 & & & & & & & & & \\
\hline Unknown lipid & 0.49 & 0.60 & 0.45 & 0.50 & & & & & & & & & \\
\hline 3-Hydroxybutyrate & & & & & 0.60 & 0.73 & 0.48 & 1.22 & & & & & Energy metabolism \\
\hline 18-Hydroxy-11-deoxycorticosterone & 0.60 & 0.73 & 0.46 & 0.67 & 0.64 & 0.65 & 0.48 & 0.68 & & & & & Hormones, signal substances and related \\
\hline Salicylic acid & 0.36 & 0.41 & 0.50 & 0.41 & & & & & & & & & Miscellaneous \\
\hline alpha-Tocopherol & 0.50 & 0.57 & 0.41 & 0.55 & 0.60 & 1.95 & 0.66 & 1.10 & & & & & Vitamins, cofactors and related \\
\hline Coenzyme Q9 & 0.33 & 0.62 & 0.46 & 0.60 & 0.68 & 0.89 & 0.39 & 0.85 & & & & & \\
\hline Coenzyme Q10 & 0.34 & 0.58 & 0.49 & 0.64 & 0.40 & 0.85 & 0.60 & 0.78 & & & & & \\
\hline
\end{tabular}




\section{Discussion}

\section{Impact of antibiotics on the metabolite profile of gut tissue and content}

The metabolite profile of different matrices of the gut after antibiotic treatment was analyzed to 1) evaluate gut microbiota metabolite changes observed at different dose levels and in different sexes, and 2) to identify the best tissue matrix for further investigations regarding an assessment of metabolic effects of new compounds with antibiotic activity. The metabolite profiles of sampled gut matrices (i.e. jejunum, ileum, rinsed cecum, colon, rectum) were different from each other, but were not affected by treatment (see supplementary material). Feces and cecum content of treated animals had significantly different metabolome profiles compared to the control. There was no dose-dependency regarding absolute numbers of altered metabolites. However, a dose-dependency could be observed for vancomycin and streptomycin in feces and cecum content.

Overall, the number of significantly upregulated metabolites was higher than the number of downregulated metabolites. This observation together with the nature of the metabolites that were upregulated suggests that due to the loss of gut bacteria certain food components were not used. Decreases in metabolite levels are likely due to a community shift and/or an imbalanced usage of nutrients. The metabolite changes in the feces and cecum content were similar, except for the roxithromycin female group where cecum content showed about $10 \%$ more upregulated and about $7 \%$ less downregulated metabolites than in feces in both dose groups. This overall similar patterns of metabolic changes in feces and cecum content is in line with the findings of Li et al. who reported that the gut community in rats is similar in cecum content and feces ( $\mathrm{Li}$ et al., 2017).

It is well known that the sex has an impact on plasma metabolite profiles and in general we observed significant differences between sexes (Strauss et al., 2009). In cecum and feces however, only minor differences between male and female samples were observed with this targeted analysis which is a first indication that regarding the analyzed metabolites there is no significant sex difference in the metabolic output of the microbiome in these matrices.

The profile strength showed that vancomycin, which is not systemically bioavailable, had the most profound effect on the metabolome of feces and cecum content followed by roxithromycin, while streptomycin had a comparably modest effect. This reflects the activity spectra of the antibiotics applied. Vancomycin, a glycopeptide antibiotic with a spectrum of activity against Gram-positive organisms has a profound effect on the gut microbiome in humans (Auwera et al., 1996; Lund et al., 2000; Vrieze et al., 2014). Roxithromycin, a bioavailable macrolide antibiotic with a narrow spectrum affecting Grampositive bacteria and some Gram-negative bacteria, showed in an investigation with volunteers no significant change of the community but a decrease of the total number of enterobacteria (Pecquet et al., 1991). In our study, the least changes in the metabolome were observed after streptomycin treatment. This poorly bioavailable antibiotic belonging to the class of aminoglycosides is only effective against aerobic organisms but not anaerobic bacteria that dominate the gut microbiome (The Human Microbiome Project Consortium, 2012). In another study, in which mice received a single dose of $20 \mathrm{mg}$ 
streptomycin an overall decrease in diversity and increase of Ruminococcaceae and Bacteroidaceae was observed (Lichtman et al., 2016).

The differences of the activity spectra and similarities of both matrices also emerge from both the calculation of the profile strength and the PCA where both feces and cecum content from males and females showed a similar pattern: streptomycin having the lowest treatment-related effects compared to vancomycin and roxithromycin. Especially vancomycin showed a dose-dependent effect over all groups and both matrices. Whether the limited or even absent dose-response behavior for the changes observed in feces and cecum for streptomycin and roxithromycin can be ascribed to saturation of the doseresponse behavior at the dose levels tested and/or to other explanations remains to be established.

\section{Metabolite changes}

With a ranking for both matrices and sexes of 25 metabolites with the highest loading in PC1, 2 and 3 the most important metabolites that drive the separation were identified (see Figure 2, Figure 3, and Figure 4). For all three antibiotic treatments, the metabolites with the highest loadings were almost all considerably upregulated for both dose groups and in both matrices. Furthermore, male and female animals showed a significant overlap of metabolites in feces and cecum content. With a separate analysis, the downregulated metabolites in the feces of males and females were analyzed. Additionally, the metabolites were analyzed which were altered after all treatments in feces and cecum content of male and female animals in the same direction. These results are discussed in detail in the following sections.

\section{Vancomycin}

Compared to roxithromycin and streptomycin, vancomycin has the most profound effect on the metabolites in both matrices in both sexes. The 25 metabolites with the highest loadings in feces were all upregulated except for cholic acid in the HD of cecum content samples which was downregulated. Highly upregulated values could be observed for creatinine and trans-4-hydroxyproline. Creatinine and creatine, that was also upregulated, were increased due to an antibiotic elimination of microbes that produce enzymes known to degrade these metabolites (Romick-Rosendale et al., 2009; Wyss and Kaddurah-Daouk, 2000). It was also shown that creatine and creatinine were elevated in feces of germfree mice (Marcobal et al., 2013). Various TAGs were also increased. Vancomycin is known to disrupt the bile acid metabolism resulting in the increase of cholic acid. Hence, the regulation of TAGs and other fatty acids was altered (Ridlon et al., 2006; Vrieze et al., 2014) and also the loss of bacteria, and with this their lipases could lead to an increase of TAGs and result in a decrease of free lipids. Various amino acids were upregulated in both matrices and sexes. A decrease could only be observed for glutamate. This is contrary to findings of Yap et al. who found a marked depletion of a range of amino acids in feces of mice after vancomycin treatment (Yap et al., 2008). Since the dose level was similar but the application period 
lasted just for two days, it is possible that in the present study after a four-week treatment the gut microbiota were far more affected so that amino acids from the food could no longer be used efficiently by the remaining microbes. Moreover, the interspecies variability might contribute to the differences. Another metabolite, i.e. pantothenic acid, that can be derived from either the food or the microbes, was also increased. Some microbial species need pantothenic acid also for growth, and a loss of these microbes would explain an increase of this vitamin (Van Eldere et al., 1996).

The decrease of metabolites belonging to the class of complex lipids, fatty acids and 18hydroxy-11-deoxycorticosterone could be due to the disruption of the bile acid metabolism or, regarding the lipids, a decrease of microbial lipases. Also alpha-tocopherol, which is present in the bile, was decreased (Hofmann, 1999). Feces samples showed a decrease of 5 oxoproline that can be further metabolized to glutamate, which was also decreased. In the phenylalanine/tyrosine catabolic pathway tyrosine is further metabolized to 4hydroxyphenylpyruvate by the tyrosine aminotransferase (Gertsman et al., 2015). Since phenylalanine was significantly increased this pathway seemed to be disrupted. Similarly, in the tryptophan metabolism tryptophan is further metabolized to IAA. Whereas tryptophan was found to be increased, the level of IAA was significantly decreased (Gertsman et al., 2015). In conclusion, the increase of amino acids is due to the loss of microbes that normally utilize these metabolites. Generally, as described by Neis et al., amino acids can be either utilized by microorganisms, or catabolized through different pathways (Neis et al., 2015). Besides that, quinones coenzyme Q9 and Q10 were decreased. In general, quinones are produced and used by microorganisms as electron carriers that are essential for cellular respiration (Collins and Jones, 1981). Here again, a decrease of these metabolites could be explained with a substantial loss of microorganisms.

\section{Roxithromycin}

Roxithromycin, a narrow spectrum antibiotic that affects Gram-positive bacteria and some Gram-negative bacteria, in general does not lead to a significant change of the anaerobic and aerobic community but resulta in a decrease of the total number of enterobacteria (Koopman et al., 1987; Pecquet et al., 1991). This was also reflected in the less altered metabolite profile and less significantly upregulated metabolites compared to the effects induced by vancomycin. Furthermore, both in feces and cecum content no dose dependency was observed. In both matrices of males and females, allantoin, taurine, taurocholic acid, creatinine and isoleucine were significantly upregulated. Allantoin is a metabolic intermediate in microbes, that is produced from uric acid and serves as a nitrogen source (Ma et al., 2016). A loss or reduction of microorganisms, especially enterobacteria, probably led to an increase of allantoin as well as of malate, creatinine and amino acids. Taurocholic acid, a primary bile acid, was highly increased pointing at a disruption of the bacterial bile acid metabolism. Normally, primary bile acids are further metabolized to secondary bile acids and partly deconjugated. 
In the feces of the male group, a more important role than in cecum content was observed for the following metabolites: ILA, lysophosphatidylcholine, myristic acid, glucose-6phosphate and aspartate. However, all of them were just significantly upregulated in the LD group of feces samples. In in vitro experiments, ILA was hypothesized to be produced by human and not microbial cells because human cells did not complete the conversion of indolepyruvate to indole-3-carboxaldehyde and probably secrete indolepyruvate and ILA back to circulation (Gertsman et al., 2015). This suggests that increased levels of ILA can be of mammalian origin. Also in the female group ILA was slightly increased in the feces but not significantly in cecum content. Also derived from the tryptophan pathway is IAA, which was downregulated just in the top 25 of feces, but only significantly in the cecum content of the LD. In both sexes, fatty acids and lipids were probably increased due to the disruption of the bile acid metabolism. Besides fatty acids and amino acids which were not observed in the top25 metabolites of feces but in cecum content, also ornithine was increased. Ornithine serves as a H-acceptor in the catabolic pathway of microbes (Davila et al., 2013). Similar to vancomycin, most of the decreased metabolites belong to the class of complex lipids and fatty acids which could be altered due to a decrease of microbial lipases or the disruption of the bile acid metabolism. This could be also true for 18-hydroxy-11deoxycorticosterone and alpha-tocopherol that were decreased in feces of female animals. Interestingly, more lipids as well as coenzyme Q10 were decreased in female animals, whereas IAA was significantly decreased only in male animals. Coenzyme 9 was decreased in both sexes. These metabolites were also found to be decreased after vancomycin treatment as discussed before.

\section{Streptomycin}

Treatment with streptomycin showed in PC1 versus PC3 but not PC2 a separation from the control for the HD groups and a slight separation of the LD group. In general, streptomycin showed modest effects on the metabolite profile which was also reflected by the low profile strength. This could result from its activity only spectra against aerobic but not anaerobic organisms, which dominate the gut microbiota (The Human Microbiome Project Consortium, 2012). However, a change or depletion of aerobic bacteria can result in a shift of the anaerobes as shown in a study reported by Lichtman et al., who observed an overall decrease in microbial diversity and increase of Ruminococcaceae and Bacteroidaceae upon treatment of mice with $20 \mathrm{mg}$ streptomycin (Lichtman et al., 2016).

Regarding the metabolites with the highest loadings in both matrices of males and females a significant increase was observed for kynurenic acid and cysteine. Also, an increase of amino acids could be observed, especially for the HD. The HD seemed to be more effective and hence could have wiped out more communities responsible for the metabolism of these amino acids.

In the male group, pantothenic acid was significantly upregulated in cecum content and feces but interestingly, higher upregulated in the LD and not significantly altered in female groups. As for vancomycin treatment, an excess of pantothenic acid could be also derived 
from a loss of microbes (Van Eldere et al., 1996). Interestingly, spermidine, which was found to be upregulated in the gut content, was decreased in the plasma after antibiotic treatment with aminoglycosides in our previous study (Behr et al., 2017). This polyamine is generated from precursor amino acids by the microbes and in low amounts by normal colonocytes (Davila et al., 2013; Ramezani et al., 2015). We suggest that spermidine is not absorbed or produced in higher rates, respectively, and hence could accumulate in the gut. Uracil was found to a higher extent in the LD. Uracil is normally absorbed in the small intestine through specific nucleoside transporters (Moran-Ramos et al., 2017). Due to perturbations of the upper part of the intestine and in the bile acid metabolism, the transporters and metabolizing enzymes might be downregulated. The effect of streptomycin on the bile acid metabolism as well as on the steroid and eicosanoid metabolism was earlier described by Antunes et al. (Antunes et al., 2011). In our study, we could also observe changes for cholic acid, taurocholic acid and glycochenodeoxycholic acid both in females and males. Interestingly, more changes of increased lipids and TAGs, especially for cecum content, could be observed in female animals. Thus, whereas stearic acid was decreased in both sexes, even more decreased metabolites were found in the female groups. This indicates that the lipid metabolism, including the microbial lipases, in females is more affected after antibiotic treatment and might suggest a sex-specific reaction or alteration of the host-microbial co-metabolism. In a study of Markle et al., sex-specific differences in the composition of the microbiome of cecum content were found at puberty and were most apparent in adult mice (Markle et al., 2013). Due to sex-specific changes in the plasma metabolome caused by a subset of glycerophospholipid and sphingolipid metabolites - not observed for the germfree mice - the authors assumed that the microbiome plays a role in regulating sex-specific features of the host metabolic profile.

In both sexes and in all dose groups of vancomycin, roxithromycin and streptomycin, stearic acid was decreased and kynurenic acid was highly increased. Kynurenic acid can either be found in the diet or is made from tryptophan and enters the kynurenine pathway which is the most tryptophan- consuming metabolic pathway in mammalian and microbial cells (Peters, 1991). However, tryptophan levels were also slightly increased in the vancomycin and roxithromycin groups but not in the streptomycin groups. The decrease of stearic acid could result from the disruption of the lipid metabolism, including the microbial lipases, which cleave the TAGs into free fatty acids. 


\section{CONCLUSION}

The results of the present paper show that metabolic profiling allows a first assessment if and to what extent the gut microbiota is affected by a substance. It was possible to distinguish three antibiotics with each a different activity spectra from each other in feces and cecum content. In gut tissue, no treatment-related effect could be observed. In line with the activity spectra of the antibiotics tested, vancomycin showed the most profound effect on the metabolite profile, followed by roxithromycin and then by streptomycin for which changes were modest. Vancomycin showed also a clear separation of the LD and HD group. In general, for all antibiotics the largest changes were observed for the classes of lipids, bile acids and amino acids. Regarding gender-related effects, with this targeted analysis all dose groups of the three antibiotics had just minor differences in the top25 metabolites between male and female samples. Furthermore, feces and cecum content showed just subtle differences of their metabolite profile within each treatment group. Concluding, taking other samples than feces does not add any extra information. Thus, as a non-invasive sampling method, feces provide a suitable matrix for studies on metabolism by the gut microbiota.

Acknowledgement: We would like to thank Ms Irmgard Weber for her skillful assistance.

Funding information: The authors have no relevant affiliations or financial involvement with any organization or entity with a financial interest in or financial conflict with the subject matter or materials discussed in the manuscript. This includes employment, consultancies, honoraria, stock ownership or options, expert testimony, grants or patents received or pending, or royalties. No writing assistance was utilized in the production of this manuscript

\section{Appendix A. Supplementary data}

Supplementary data to this article can be found online at https:// doi.org/10.1016/j.taap.2018.06.028. 


\section{REFERENCES}

Antunes, L.C.M., Han, J., Ferreira, R.B.R., Lolić, P., Borchers, C.H., Finlay, B.B., 2011. Effect of antibiotic treatment on the intestinal metabolome. Antimicrob. Agents Chemother. 55, 1494-1503. https://doi.org/10.1128/AAC.01664-10

Auwera, P. Van der, Pensart, N., Korten, V., Murray, B.E., Leclercq, R., 1996. Influence of oral glycopeptides on the fecal flora of human volunteers: selection of highly glycopeptide-resistant enterococci. J. Infect. Dis. 173, 1129-1136. https://doi.org/10.1093/infdis/173.5.1129

Behr, C., Kamp, H., Fabian, E., Krennrich, G., Mellert, W., Peter, E., Strauss, V., Walk, T., Rietjens, I.M.C.M., van Ravenzwaay, B., 2017. Gut microbiome-related metabolic changes in plasma of antibiotic-treated rats. Arch. Toxicol. 91, 3439-3454. https://doi.org/10.1007/s00204-017-1949-2

Cho, I., Blaser, M.J., 2012. The human microbiome: at the interface of health and disease. Nat. Rev. Genet. 13, 260-70. https://doi.org/10.1038/nrg3182

Collins, M.D., Jones, D., 1981. Distribution of isoprenoid quinone structural types in bacteria and their taxonomic implication. Microbiol. Rev. 45, 316-54.

David, L. a, Maurice, C.F., Carmody, R.N., Gootenberg, D.B., Button, J.E., Wolfe, B.E., Ling, A. V, Devlin, a S., Varma, Y., Fischbach, M. a, Biddinger, S.B., Dutton, R.J., Turnbaugh, P.J., 2014. Diet rapidly and reproducibly alters the human gut microbiome. Nature 505, 559-63. https://doi.org/10.1038/nature12820

Davila, A.M., Blachier, F., Gotteland, M., Andriamihaja, M., Benetti, P.H., Sanz, Y., Tomé, D., 2013. Re-print of "intestinal luminal nitrogen metabolism: Role of the gut microbiota and consequences for the host." Pharmacol. Res. 69, 114-126. https://doi.org/10.1016/j.phrs.2013.01.003

Fernie, A.R., Trethewey, R.N., Krotzky, A.J., Willmitzer, L., 2004. Metabolite profiling: from diagnostics to systems biology. Nat. Rev. Mol. Cell Biol. 5, 763-9. https://doi.org/10.1038/nrm1451

Gertsman, I., Gangoiti, J.A., Nyhan, W.L., Barshop, B.A., 2015. Perturbations of tyrosine metabolism promote the indolepyruvate pathway via tryptophan in host and microbiome. Mol. Genet. Metab. 114, 431-437. https://doi.org/10.1016/j.ymgme.2015.01.005

Heinken, A., Thiele, I., 2015. Systems biology of host-microbe metabolomics. Wiley Interdiscip. Rev. Syst. Biol. Med. 7, 195-219. https://doi.org/10.1002/wsbm.1301

Hofmann, A.F., 1999. Bile Acids: The Good, the Bad, and the Ugly. News Physiol. Sci. $14,24-29$.

Kamp, H., Strauss, V., Wiemer, J., Leibold, E., Walk, T., Mellert, W., Looser, R., Prokoudine, A., Fabian, E., Krennrich, G., Herold, M., Ravenzwaay, B. van, 2012. Reproducibility and robustness of metabolome analysis in rat plasma of 28-day repeated dose toxicity studies. Toxicol. Lett. 215, 143-149.

Koopman, J.P., Van den Brink, M.E., Scholten, P.M., Hectors, M.P.C., Nagengast, F.M., 1987. Influence of the antibiotics roxithromycin and erythromycin on the gastrointestinal ecology of mice. Zeitschrift fuer Versuchstierkd. 30, 79-83.

Li, D., Chen, H., Mao, B., Yang, Q., Zhao, J., Gu, Z., Zhang, H., Chen, Y.Q., Chen, W., 
2017. Microbial Biogeography and Core Microbiota of the Rat Digestive Tract. Sci. Rep. 8:45840. https://doi.org/10.1038/srep45840

Lichtman, J.S., Ferreyra, J.A., Ng, K.M., Smits, S.A., Sonnenburg, J.L., Elias, J.E., 2016. Host-Microbiota Interactions in the Pathogenesis of Antibiotic-Associated Diseases. Cell Rep. 14, 1049-1061. https://doi.org/10.1016/j.celrep.2016.01.009

Lindon, J.C., Holmes, E., Bollard, M.E., Stanley, E.G., Nicholson, J.K., 2004. Metabonomics technologies and their applications in physiological monitoring, drug safety assessment and disease diagnosis. Biomarkers 9, 1-31. https://doi.org/10.1080/13547500410001668379

Lund, B., Edlund, C., Barkholt, L., Nord, C.E., Tvede, M., Poulsen, R.L., 2000. Impact on human intestinal microflora of an Enterococcus faecium probiotic and vancomycin. Scand. J. Infect. Dis. 32, 627-632. https://doi.org/10.1080/003655400459531

Ma, P., Patching, S.G., Ivanova, E., Baldwin, J.M., Sharples, D., Baldwin, S.A., Henderson, P.J.F., 2016. Allantoin transport protein, Pucl, from Bacillus subtilis: Evolutionary relationships, amplified expression, activity and specificity. Microbiol. (United Kingdom) 162, 823-836. https://doi.org/10.1099/mic.0.000266

Marcobal, a, Kashyap, P.C., Nelson, T. a, Aronov, P. a, Donia, M.S., Spormann, a, Fischbach, M. a, Sonnenburg, J.L., 2013. A metabolomic view of how the human gut microbiota impacts the host metabolome using humanized and gnotobiotic mice. ISME J. 7, 1933-43. https://doi.org/10.1038/ismej.2013.89

Markle, J.G.M., Frank, D.N., Mortin-toth, S., Robertson, C.E., Feazel, L.M., Rollekampczyk, U., Bergen, M. Von, Mccoy, K.D., Macpherson, A.J., Danska, J.S., 2013. Sex Differences in the Gut Microbiome Drive Hormone-Dependent Regulation of Autoimmunity. Science (80-. $\quad$ ). 339, 1084-1088. https://doi.org/10.1126/science.1233521

McCoy, K.D., Geuking, M.B., Ronchi, F., 2017. Gut microbiome standardization in control and experimental mice. Curr. Protoc. Immunol. 2017, 23.1.1-23.1.13. https://doi.org/10.1002/cpim.25

Modi, S.R., Collins, J.J., Relman, D. a, 2014. Antibiotics and the gut microbiota. J. Clin. Invest. 124, 4212-4218. https://doi.org/10.1172/JCI72333.themselves

Moran-Ramos, S., He, X., Chin, E.L., Tovar, A.R., Torres, N., Slupsky, C.M., Raybould, H.E., 2017. Nopal feeding reduces adiposity, intestinal inflammation and shifts the cecal microbiota and metabolism in high-fat fed rats. PLoS One 12, e0171672. https://doi.org/10.1371/journal.pone.0171672

Neis, E.P.J.G., Dejong, C.H.C., Rensen, S.S., 2015. The Role of Microbial Amino Acid Metabolism in Host Metabolism. Nutrients 7, 2930-2946. https://doi.org/10.3390/nu7042930

Nguyen, T.L.A., Vieira-Silva, S., Liston, A., Raes, J., 2015. How informative is the mouse for human gut microbiota research? Dis. Model. Mech. 8, 1-16. https://doi.org/10.1242/dmm.017400

Pecquet, S., Chachaty, E., Tancrede, C., Andremont, A., 1991. Effects of roxithromycin on fecal bacteria in human volunteers and resistance to colonization in gnotobiotic mice. Antimicrob. Agents Chemother. 35, 548-552. 
Peters, J.C., 1991. Tryptophan nutrition and metabolism: an overview, in: Kynurenine and Serotonin Pathways. pp. 345-358. https://doi.org/10.1007/978-1-4684-5952-4_32

Ramezani, A., Massy, Z. a., Meijers, B., Evenepoel, P., Vanholder, R., Raj, D.S., 2015. Role of the Gut Microbiome in Uremia: A Potential Therapeutic Target. Am. J. Kidney Dis. 1-16. https://doi.org/10.1053/j.ajkd.2015.09.027

Ridlon, J.M., Kang, D.-J., Hylemon, P.B., 2006. Bile salt biotransformations by human intestinal bacteria. J. Lipid Res. 47, 241-259. https://doi.org/10.1194/jlr.R500013JLR200

Roessner, U., Wagner, C., Kopka, J., Trethewey, R.N., Willmitzer, L., 2000. Technical advance: simultaneous analysis of metabolites in potato tuber by gas chromatography-mass spectrometry. Plant J. 23, 131-42. https://doi.org/10.1046/j.1365-313x.2000.00774.x

Romick-Rosendale, L.E., Goodpaster, A.M., Hanwright, P.J., Patel, N.B., Wheeler, E.T., Chona, D.L., Kennedy, M. a., 2009. NMR-based metabonomics analysis of mouse urine and fecal extracts following oral treatment with the broad-spectrum antibiotic enrofloxacin (Baytril). Magn. Reson. Chem. 47, S36-S46. https://doi.org/10.1002/mrc.2511

Rooks, M.G., Garrett, W.S., 2016. Gut microbiota, metabolites and host immunity. Nat. Rev. Immunol. 16, 341-52. https://doi.org/10.1038/nri.2016.42

Rosenfeld, C.S., 2017. Gut Dysbiosis in Animals Due to Environmental Chemical $\begin{array}{lllll}\text { Exposures. } & \text { Front. } & \text { Cell. } & \text { Infect. } & \text { Microbiol. }\end{array}$ https://doi.org/10.3389/fcimb.2017.00396

Sender, R., Fuchs, S., Milo, R., 2016. Revised estimates for the number of human and bacteria cells in the body. PLoS Biol. 14. https://doi.org/10.1101/036103

Silbergeld, E.K., 2017. The Microbiome. Toxicol. Pathol. 45, 190-194. https://doi.org/10.1177/0192623316672073

Strauss, V., Mellert, W., Wiemer, J., Leibold, E., Kamp, H., Walk, T., Looser, R., Prokoudine, a., Fabian, E., Krennrich, G., Herold, M., Van Ravenzwaay, B., 2012. Increased toxicity when fibrates and statins are administered in combination - A metabolomics approach with rats. Toxicol. Lett. 211, 187-200. https://doi.org/10.1016/j.toxlet.2012.03.798

Strauss, V., Wiemer, J., Leibold, E., Kamp, H., Walk, T., Mellert, W., Looser, R., Prokoudine, a., Fabian, E., Krennrich, G., Herold, M., van Ravenzwaay, B., 2009. Influence of strain and sex on the metabolic profile of rats in repeated dose toxicological studies. Toxicol. Lett. 191, 88-95. https://doi.org/10.1016/j.toxlet.2009.08.004

The Human Microbiome Project Consortium, 2012. Structure, function and diversity of the healthy human microbiome, Nature. Nature Publishing Group. https://doi.org/10.1038/nature11234

Van Eldere, J., Celis, P., De Pauw, G., Lesaffre, E., Eyssen, H., 1996. Tauroconjugation of cholic acid stimulates 7 alpha-dehydroxylation by fecal bacteria. Appl. Environ. Microbiol. 62, 656-61.

van Ravenzwaay, B., Coelho-Palermo Cunha, G., Strauss, V., Wiemer, J., Leibold, E., 
Kamp, H., Walk, T., Mellert, W., Looser, R., Prokoudine, a., Fabian, E., Krennrich, G., Herold, M., 2010. The individual and combined metabolite profiles (metabolomics) of dibutylphthalate and di(2-ethylhexyl)phthalate following a 28-day dietary exposure in rats. Toxicol. Lett. 198, 159-170. https://doi.org/10.1016/j.toxlet.2010.06.009

van Ravenzwaay, B., Cunha, G.C.P., Leibold, E., Looser, R., Mellert, W., Prokoudine, a., Walk, T., Wiemer, J., 2007. The use of metabolomics for the discovery of new biomarkers of effect. Toxicol. Lett. 172, 21-28. https://doi.org/10.1016/j.toxlet.2007.05.021

van Ravenzwaay, B., Herold, M., Kamp, H., Kapp, M.D., Fabian, E., Looser, R., Krennrich, G., Mellert, W., Prokoudine, A., Strauss, V., Walk, T., Wiemer, J., 2012. Metabolomics: A tool for early detection of toxicological effects and an opportunity for biology based grouping of chemicals-From QSAR to QBAR. Mutat. Res. - Genet. Toxicol. Environ. Mutagen. 746, 144-150. https://doi.org/10.1016/j.mrgentox.2012.01.006

van Ravenzwaay, B., Kamp, H., Montoya-parra, G.A., Strauss, V., Fabian, E., Mellert, W., Walk, T., Peter, E., Looser, R., Herold, M., 2016. The development of a database for metabolomics - looking back on ten years of experience. Int. J. Biotechnol. 14, 4768. https://doi.org/10.1504/IJBT.2015.074801

Vrieze, A., Out, C., Fuentes, S., Jonker, L., Reuling, I., Kootte, R.S., Van Nood, E., Holleman, F., Knaapen, M., Romijn, J. a., Soeters, M.R., Blaak, E.E., Dallinga-Thie, G.M., Reijnders, D., Ackermans, M.T., Serlie, M.J., Knop, F.K., Holst, J.J., Van Der Ley, C., Kema, I.P., Zoetendal, E.G., De Vos, W.M., Hoekstra, J.B.L., Stroes, E.S., Groen, A.K., Nieuwdorp, M., 2014. Impact of oral vancomycin on gut microbiota, bile acid metabolism, and insulin sensitivity. J. Hepatol. 60, 824-831. https://doi.org/10.1016/j.jhep.2013.11.034

Walk, T.B., Dostler, M., 2003. Mass spectrometry method for analysing mix- tures of substances, Patent application PCT/EP2003/001274. WO2003073464, Metanomics, Berlin.

Walk, T.B., Looser, R., Bethan, B., Herold, M.M., Kamlage, B., Schmitz, O., van Ravenzwaay, B., Mellert, W., Coelho, P.C.G., Ehrhardt, T., Wiemer, J., Prokoudine, A., Krennrich, G., 2006. Means and methods for analyzing a sample by means of chromatography-mass spectrometry, Patent application PCT/EP2006/064628. WO2007012643A1, Metanomics, Berlin.

Wikoff, W.R., Anfora, A.T., Liu, J., Schultz, P.G., Lesley, S. a, Peters, E.C., Siuzdak, G., 2009. Metabolomics analysis reveals large effects of gut microflora on mammalian blood metabolites. Proc. Natl. Acad. Sci. U. S. A. 106, 3698-3703. https://doi.org/10.1073/pnas.0812874106

Wyss, M., Kaddurah-Daouk, R., 2000. Creatine and Creatinine Metabolism. Physiol. Rev. 80, 1107-1213. https://doi.org/10.1016/S1286-0115(06)74505-2

Yap, I.K.S., Li, J. V, Saric, J., Martin, F.-P., Davies, H., Wang, Y., Wilson, I.D., Nicholson, J.K., Marchesi, J.R., Holmes, E., Utzinger, J., Marchesi, J.R., Holmes, E., 2008. Metabonomic and Microbiological Analysis of the Dynamic Effect of VancomycinInduced Gut Microbiota Modification in the Mouse. J. Proteome Res. 7, 3718-3728. https://doi.org/10.1021/pr700864x 
Chapter 3

Zhang, Y.-J., Li, S., Gan, R.-Y., Zhou, T., Xu, D.-P., Li, H.-B., 2015. Impacts of Gut Bacteria on Human Health and Diseases. Int. J. Mol. Sci. 16, 7493-7519. https://doi.org/10.3390/ijms16047493 


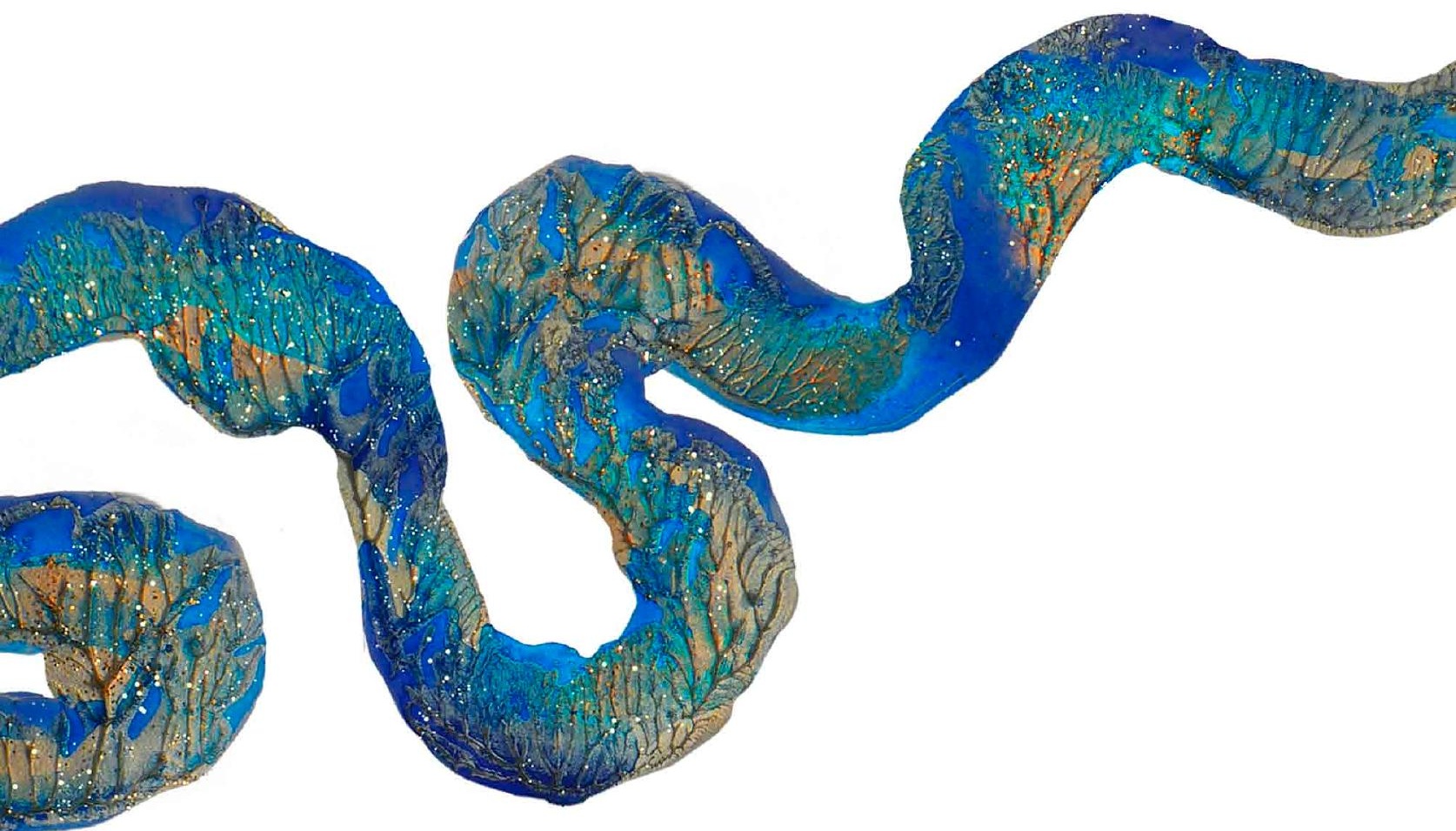




\section{Chapter 4}

Impact of lincosamides antibiotics on the composition of the rat gut microbiota and the metabolite profile of plasma and feces

C. Behr, S. Ramírez-Hincapié, H.J. Cameron, V. Strauss, T. Walk, M. Herold, K. Beekmann, I.M.C.M. Rietjens, B. van Ravenzwaay

Published in:

Toxicology Letters 296 (2018), 139-151 


\section{ABSTRACT}

The importance of the gut microorganisms and their wide range of interactions with the host are well-acknowledged. In this study, lincomycin and clindamycin were used to modulate microbial communities of Wistar rats to gain a comprehensive understanding of the implications of microbiome alterations. A metabolomics approach and taxonomic profiling were applied to characterize the effects of these antibiotics on the functionality of the microbiome and to identify microbiome-related metabolites. After treatment, the diversity of the microbial community was drastically reduced. Bacteroidetes and Verrucomicrobia were drastically reduced, Tenericutes and Deferribacteres completely disappeared, while abundance of Firmicutes and Proteobacteria were highly increased. Changes in plasma and feces metabolites were observed for metabolites belonging mainly to the class of complex lipids, fatty acids and related metabolites as well as amino acids and related compounds. Bile acid metabolism was markedly affected: taurocholic acid, glycochenodeoxycholic acid and cholic acid presented abrupt changes showing a specific metabolite pattern indicating disruption of the microbial community. In both plasma and feces taurocholic acid was highly upregulated upon treatment whereas glycochenodeoxycholic acid was downregulated. Cholic acid was upregulated in feces but downregulated in plasma. These results show that changes in the gut microbial community lead to alterations of the metabolic profile in blood and feces of the host and can be used to identify potentially microbiome-related metabolites. This implies that metabolomics could be a suitable tool to estimate the extent of changes induced in the intestinal microbiome with respect to consequences for the host. 
Key words: metabolomics, gut microbiome, taxonomic profiling, repeated dose oral toxicity study, antibiotics, microbiome-related metabolites

$\begin{array}{ll}\text { ABBREVIATIONS } \\ \text { ANCOM } & \text { analysis of composition of microbiomes } \\ \text { BSH } & \text { bile salt hydrolase } \\ \text { GC } & \text { gas chromatography } \\ \text { DAG } & \text { diacylglycerine } \\ \text { GF } & \text { germ-free } \\ \text { IBD } & \text { inflammatory bowel disease } \\ \text { LC } & \text { liquid chromatography } \\ \text { LD } & \text { low dose } \\ \text { MOA } & \text { mode of action } \\ \text { MS } & \text { mass spectrometry } \\ \text { OECD } & \text { Organisation for Economic Co-operation and Development } \\ \text { PCA } & \text { principal component analysis } \\ \text { PCoA } & \text { principal coordinate analysis } \\ \text { SPE } & \text { solid phase extraction } \\ \text { TAG } & \text { triacylglycerol }\end{array}$

\section{INTRODUCTION}

The gut microbiome has gained a lot of attention in the last years, becoming a rapidly growing field of study. It is increasingly recognized as a critical component in human development, health and disease and it is gaining a spot in fields such as oncology, toxicology, pharmacology, neurology and many others. Next generation sequencing and the development of metagenomic tools allow to characterize the gut microorganisms and have shed light on their importance for the host system (Gilbert et al., 2016). Although the fundamental role of the microbiome in the well-being of the host is clear, science is now dealing with a bigger challenge: i.e. understanding the mechanisms by which this process is driven. Integrative approaches that not only asses the composition of the gut microbiome but also its functionality must be implemented to unravel the complex dynamics of hostmicrobiome interactions (Bäumler and Sperandio, 2016). There are high intra- and interspecies variabilities regarding the composition of the microbiome, however, there are also indications that the functionality in terms of gene activity and metabolic output of the microbes have a lower variability (Cho and Blaser, 2012; McCoy et al., 2017) which supports assessing functional microbial changes with metabolomics approaches. Currently, metabolomics is increasingly used to study the gut microbiome since matrices like urine, blood or feces can be screened, which also enables a longitudinal study design and the analysis of metabolic fluxes. Recent research has demonstrated the large impact the gut microbiome has on mammalian blood metabolites, suggesting a major interplay between 
bacterial and mammalian metabolism (Wikoff et al., 2009). Metabolites produced or modified by the gut microbiota can be absorbed across the intestinal cell wall and can therefore be detected in plasma. Numerous circulating molecules have been determined to arise exclusively in the presence of gut microflora (Wikoff et al., 2009).

Initial investigations demonstrated the identification of different modes of action (MoAs) or adverse outcomes after substance treatment using plasma metabolomics in rats (van Ravenzwaay et al., 2007). It was noted that the chemicals or diseases that produce a specific form of toxicity, generally through a shared mode of action, produced a subset of common specific metabolite changes. Such a common set of consistently regulated metabolites can be used to establish metabolic patterns specific for certain toxicities (Kamp et al., 2012; Mattes et al., 2014). Using this approach, microbiome-related metabolites in plasma could previously be identified after antibiotic treatment and specific metabolite patterns could be established in the MetaMap®Tox (Behr et al., 2017), a metabolomics database for the identification and prediction of toxicological modes of action (MoAs) of new test substances.

In a previous study, we performed metabolite profiling of different matrices of the gut of rats, after antibiotic treatment in order to evaluate metabolite changes observed at different dose levels and in different sexes, and to identify the best tissue matrix for further investigations. The results of this targeted analysis showed that the metabolic profiles of male and female animals in the gut microbiome are comparable and that taking other samples (e.g. from different regions of the gastrointestinal tract) than feces did not add any extra information (Behr et al, submitted). Besides, the metabolites found in feces were similar to those in cecum content indicating that feces sampling for in vitro incubations could provide a suitable non-invasive method for studying microbiome related metabolites and metabolism.

In this study, we analyzed the same metabolites in both plasma and feces to evaluate the effect of gut microbial changes on the plasma metabolome. Assessing the metabolite changes in the feces after the administration of lincosamides can support the evaluation of the antibiotics effects on the microbiome regarding its functionality. The aim of this study was to establish specific plasma metabolome patterns for the effects of lincosamides on the microbiome using the MetaMap ${ }^{\circledR T o x}$ database, and to identify potential microbiomeassociated or -derived metabolites in plasma based on changes of the gut core diversity of lincosamides-treated rats. Therefore, the feces metabolome was evaluated after the antibiotic treatment to aid in the establishment of more accurate plasma patterns and to gain a more comprehensive understanding of the effects and dynamics of microbiome alterations. 


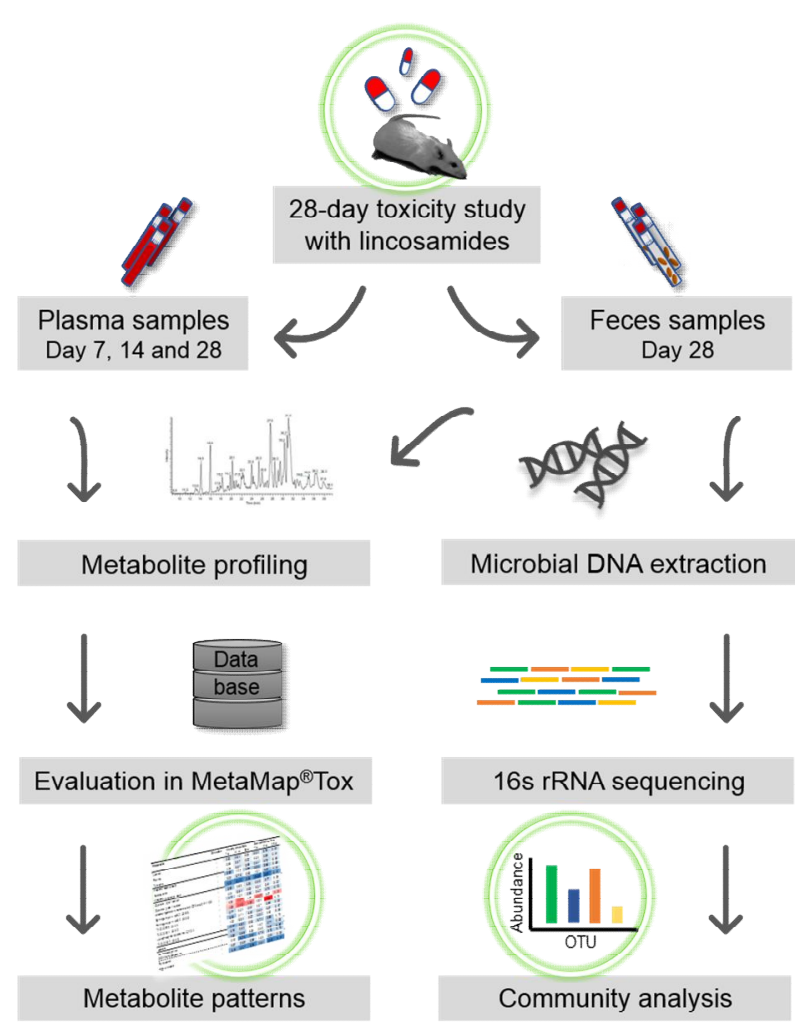

Figure 1 Schematic presentation of the study design for metabolite profiling in plasma and feces of antibiotic treated rats and the corresponding community analysis. Clindamycin and lincomycin were administered orally for 28 days to rats. Blood samples were taken after 7, 14 and 28 days for metabolite profiling. Feces samples were taken at the last day of the study for metabolite profiling and targeted metagenomics analysis. The aim was to identify metabolites related to gut microbial changes seen in the community analysis due to antibiotic treatment and to establish specific plasma metabolome patterns in the MetaMap ${ }^{\circledR}$ Tox database.

\section{Materials ANd Methods}

Three independent studies were performed according to the Organisation for Economic Cooperation and Development (OECD) 407 protocol. Animal handling, treatment, and clinical examinations have been described earlier (Kamp et al., 2012; Strauss et al., 2012; van Ravenzwaay et al., 2012, 2010, 2007).

\section{Ethics statement}

The studies were approved by the BASF Animal Welfare Body, with the permission of the local authority, the Landesuntersuchungsamt Rheinland-Pfalz (approval number 23177 07/G 13-3-016). The study was performed in an AAALAC-approved (Association for Assessment and Accreditation of Laboratory Animal Care International) laboratory in accordance with the German Animal Welfare Act and the effective European Council Directive. 


\section{Animals and Maintenance Conditions}

Briefly, male and female Wistar rats (CrI:WI(Han)) were supplied by Charles River, Germany, and were $70 \pm 1$ days old at the beginning of the studies. The animals ( 5 rats per sex and cage in one group) were maintained in an air-conditioned room at a temperature of 20 to $24^{\circ} \mathrm{C}$, a relative humidity of 30 to $70 \%$, and a 12 hour light / 12 hour dark cycle. Ground Kliba mouse/rat maintenance diet "GLP" was supplied by Provimi Kliba SA, Kaiseraugst, Switzerland. Diet and drinking water were available ad libitum (except 16 - 20 hours before sampling) and regularly assayed for chemical contaminants and the presence of microorganisms.

\section{Treatment of animals with compounds}

The antibiotics were administered daily by gavage to each treatment group consisting of five rats per group and sex. Clindamycin was prepared in ultrapure water and given at concentrations of $200 \mathrm{mg} / \mathrm{kg}$ body weight. Lincomycin was given at $300 \mathrm{mg} / \mathrm{kg}$ body weight in water containing $0.5 \%$ carboxymethyl cellulose (CMC). Dose-levels were selected according the literature data (Gray et al., 1972, 1971, 1966, 1964).To examine that the effect of $\mathrm{CMC}$ does not interfere with metabolite changes in the plasma patterns arisen from antibiotic treatment, 10 animals per sex received $10 \mathrm{ml} / \mathrm{kg}$ bw/d drinking water containing $0.5 \% \mathrm{CMC}$. The standard procedure for the evaluation of test substance-related effects in the MetaMap®Tox database is the comparison of the resulting values of the treatment groups against the baseline values of a diet control. This procedure has been used since 2004 resulting in a very extensive database of control values for the metabolites evaluated. As described in van Ravenzwaay et al (van Ravenzwaay et al., 2016), this gives us the ability to determine not only the statistical significance of regulated metabolites, but also allows the assessment if a particular metabolite value has ever been observed in control animals - providing a historical range of what is normal. The three substances (clindamycin, lincomycin and CMC) were each administered in separate studies, each with a diet control group of 10 animals per sex, to allow for such comparisons.

\section{Sampling of blood and feces for metabolite profiling}

Between 7:30 and 10:30 h, on study days 7, 14 and 28 blood samples were taken from the retro-orbital sinus in all rats under isoflurane anesthesia (1.0 $\mathrm{ml} \mathrm{K-EDTA} \mathrm{blood).} \mathrm{The} \mathrm{blood}$ samples were centrifuged $\left(10^{\circ} \mathrm{C}, 20,000 \mathrm{x} \mathrm{g}, 2 \mathrm{~min}\right)$ and the EDTA plasma was separated. The EDTA plasma samples were covered with nitrogen and frozen at $-80{ }^{\circ} \mathrm{C}$ until metabolite profiling was performed.

Feces samples were carefully removed during necropsy on day 28 from the rectum after a fasting period of $16-20$ hours and one day after the last administration of the test substances. The samples were collected in pre-cooled (dry-ice) vials, immediately snapfrozen in liquid nitrogen and stored at $-80^{\circ} \mathrm{C}$ until metabolite profiling or DNA extraction, respectively, was performed. 


\section{Clinical Examinations}

All animals were checked daily for any clinically abnormal signs and mortalities. Food consumption was determined on study days 6, 13 and 27. Body weight was determined before the start of the administration period in order to randomize the animals and on study days 6, 13 and 27. At the end of the treatment period, the animals were sacrificed by decapitation under isoflurane anesthesia. Metabolite profiling of plasma and feces samples as well as the gut microbial community analysis were performed for all control and treated animals.

\section{DNA extraction and bacterial 16S rRNA amplicon sequencing}

DNA was isolated from the fecal samples using InnuPREP stool DNA Kit (Analytik Jena) according to the manufacturer's instructions. Based on observations made during the process, the incubation temperature for the cells' lysis was lowered to $75^{\circ} \mathrm{C}$. DNA yield and integrity were assessed using a Nanodrop. Samples were sent to IMGM $^{\circledR}$ laboratories (Martinsried, Germany) for PCR, library preparation, and sequencing. DNA was amplified using 16S V3-V4 primers (Bakt_341F :5'-CCTACGGGNGGCWGCAG-3' and Bakt_805r :5'-GACTACHVGGGTATCTAATCC-3'). Sequencing was performed on the Illumina MiSeq ${ }^{\circledR}$ next generation sequencing system (Illumina Inc.). Signals were processed to fastq files and the resulting 2 × 250 bp reads were demultiplexed using the MiSeq ${ }^{\circledR R e p o r t e r}$ software.

\section{Metabolite Profiling in plasma and feces}

For mass spectrometry-based metabolite profiling, plasma and feces samples were extracted by a proprietary method as described below. GC-MS (gas chromatography-mass spectrometry) and LC-MS/MS (liquid chromatography-MS/MS) were used for broad profiling (van Ravenzwaay et al., 2007). Proteins were removed from $60 \mu \mathrm{L}$ plasma samples by precipitation using $200 \mu \mathrm{L}$ acetonitrile. Subsequently, polar and non-polar fractions were separated for both GC-MS and LC-MS/MS analysis by adding water and a mixture of ethanol and dichloromethane (1:2, v:v). Feces samples were freeze dried and ground prior to extraction. Processed feces $(5 \mathrm{mg})$ were extracted with a mixture of acetonitrile, water, ethanol and dichloromethane in a sample tube with a $3 \mathrm{~mm}$ stainless steel ball using a Bead Ruptor (Omni International Inc.). Phase separation was achieved by adding additional dichloromethane. For GC-MS analysis (CTC GC PAL, Agilent 6890 GC gas chromatograph, 5973 MSD mass spectrometer), the non-polar fraction was treated with methanol under acidic conditions to yield the fatty acid methyl esters derived from both free fatty acids and hydrolyzed complex lipids. The non-polar and polar fractions were further derivatized with O-methyl-hydroxylamine hydrochloride and pyridine to convert oxo-groups to O-methyl-oximes and subsequently with a silylating agent before analysis (Roessner et al., 2000). For LC-MS analysis, both fractions were reconstituted in appropriate solvent mixtures. HPLC was performed by gradient elution on reversed phase separation columns. Mass spectrometric detection technology was applied which allows 
target and high sensitivity MRM (Multiple Reaction Monitoring) profiling in parallel to a full screen analysis as described in patent WO2003073464 (Walk and Dostler, 2003). For GC-MS the acquisition in scan mode m/z 15-600 for polar compounds and m/z 40-600 for lipid compounds was applied. For LC-MS MRM and a Q3 Scan of m/z 100-1000 was used. MRMs off all analytes were determined using solutions of the authentic standard.

GC-MS conditions: CTC GC PAL, Agilent 6890 GC gas chromatograph, 5973 MSD mass spectrometer, gradient: $70{ }^{\circ} \mathrm{C}$ to $340{ }^{\circ} \mathrm{C}$, carrier gas: helium, acquisition in scan mode $\mathrm{m} / \mathrm{z}$ 15-600 (polar compounds) / m/z 40-600 (lipid compounds).

LC-MS conditions: Agilent 1100 HPLC System, AB Sciex API 4000 mass spectrometer, gradient elution for polar compounds with water / acetonitril / ammonium formate, gradient elution for lipid compounds with water / methanol / methyl tert-butyl ether / formic acid, MRM and Q3 Scan m/z 100-1000.

For GC-MS and LC-MS/MS profiling, data were normalized to the median of reference samples which were derived from a pool formed from aliquots of all samples to account for inter- and intra-instrumental variation. In plasma, 224 semi-quantitative metabolites could be analyzed using the single peak signal of the respective metabolite and a normalization strategy according to the patent WO2007012643A1 (Walk et al., 2006) resulting in ratio values which represent the metabolite change of treated versus control animals, of which 195 are chemically identified and 29 are structurally unknown. In feces, 208 semiquantitative metabolites could be analyzed, 177 of which are chemically identified and 31 are structurally unknown.

\section{Statistics}

The data were analyzed by univariate and multivariate statistical methods. The sex- and day-stratified heteroscedastic t-test ("Welch test") was applied to compare metabolite levels of dose groups with respective controls. For all metabolites, changes were calculated as the ratio of the mean of metabolite levels in individual rats in a treatment group relative to mean of metabolite levels in rats in a matched control group (time point, dose level, and sex). Hereafter, these ratios are referred to as 'relative abundance' in plasma or feces.

The p-values, t-values, and ratios of corresponding group medians were collected as metabolic profiles and fed into the MetaMap®Tox database (van Ravenzwaay et al., 2016). In the database, all treatment groups are compared to diet controls of the corresponding study. In this study, the profile strength of the metabolic profile is addressed. This parameter represents the rounded down average of absolute medians of t-values and does not only include the absolute number of significantly changed metabolites, but also the magnitude of the respective changes.

Briefly, the best balance for finding the maximum number of truly regulated metabolites while minimizing the number of false positive regulated metabolites is obtained at a $p$ value of 0.15 . Therefore, for pattern recognition $p$ values between 0.1 and 0.2 are used in the database for plasma. For a detailed description on the use of statistics in MetaMap ${ }$ Tox see 
van Ravenzwaay et al. (van Ravenzwaay et al., 2016). The patterns of the lincosamides were established using a p-value of 0.2 .

An in-house $\mathrm{R}$ script was written to check the completeness of the measured metabolite data. Metabolite profiles for which the database contained more than $50 \%$ of the missing values across the measured samples were removed from the analysis. As Singular Value Decomposition (SVD) PCA algorithm can only be performed on complete matrices all missing values are replaced by values computed using SVDimpute Algorithm as proposed by Troyanskaya et al. (Troyanskaya et al., 2001). PCA function from pcaMethods (Stacklies et al., 2007) R package version 1.70.0 was used with "UV" unit variance scaling option and center option set to "TRUE" to make the matrix mean centred. First 5 PCA axis was visualised using scatterD3 $\mathrm{R}$ package version 0.8.2. Whenever we write "significantly", "statistically significantly" is meant.

\section{Bioinformatics}

Forward and reverse primers corresponding to the sequences 5'CCTACGGGNGGCWGCAG-3' and 5'-GACTACHVGGGTATCTAATCC-3' were trimmed from the raw reads using cutadapt (Martin, 2011) and forward/reverse read pairs that did not contain either primer were removed. A table of amplicon sequence variants (ASV) was obtained by denoising using QIIME2's dada2 denoise-single command (Callahan et al., 2016; “QIIME2," n.d.). A phylogenetic tree was built from the ASVs using QIIME2's alignment mafft, alignment mask, phylogeny fasttree, and phylogeny midpointroot commands. Taxonomy was assigned to ASV sequences using sintax (Edgar, 2016) on version 128 of the SILVA Living Tree Project database (Yilmaz et al., 2014). Diversity analysis was performed using the diversity plugins from QIIME2 (Caporaso et al., 2010).

Statistically significant changes of the microbial community up to the taxonomic family level were evaluated with analysis of composition of microbiomes (ANCOM) as implemented in QIIMES2's composition plugin (Mandal et al., 2015).

For establishing the pairwise Krona plots, two samples of interest (A and B) were selected and an average of each taxon $(\mathrm{A}+\mathrm{B}) / 2$ was calculated, e.g. if $\mathrm{A}=20 \%$ and $\mathrm{B}=30 \%$ of Bacteroidetes; it shows up as $25 \%$ Bacteroidetes. The difference (diff) is calculated per taxa by subtracting $B$ from $A$ and reported as a difference in fraction of the total for that taxa with $\operatorname{diff}=(\mathrm{A}-\mathrm{B}) /(\mathrm{A}+\mathrm{B})$. For $\mathrm{A}=20 \%$ and $\mathrm{B}=30 \%$, the difference is -0.2 . I.e. when the second sample contributes more, the difference is negative and represents the additional fraction of that taxa that B contributed. When the first sample contributes more, the difference is positive and represents the additional fraction of that taxa that A contributed. When taxa contributions are the same, the difference is 0 .

\section{Establishment of specific metabolite patterns in MetaMap ${ }^{\circledR}$ Tox}

Patterns correlating to specific toxicological modes of action were established in MetaMap ${ }^{\circledR}$ Tox database as described before (Kamp et al., 2012). In the current study, 
patterns were created based on the metabolic profiles of clindamycin and lincomycin. Briefly, metabolites which were statistically significantly regulated in the same direction (higher/lower levels compared to controls) for both substances were identified from the MetaMap ${ }^{\circledR}$ Tox database to establish a metabolite profile. After identification of the significantly changed metabolites and a consistency check through an expert panel of experienced toxicologists, the pattern was validated against the database and should identify compounds in the MetaMap ${ }^{\circledR}$ Tox with the same toxicological mode of action.

\section{Results}

\section{Clinical signs}

No clinical signs of toxicity or mortality were observed in any of the animals that received $\mathrm{CMC}$ or lincomycin. The group of female animals treated with clindamycin showed temporarily following relevant clinical signs salivation and semi closed eyelid (4 animals), and one of the animals presented fur piloerection. Similarly, males treated with clindamycin, showed slight salivation (all animals), semi closed eyelid ( 3 animals) and, two of them were in a general poor condition. Except for salivation, these findings were only observed in the individual animals on 1-3 days of the administration period of 28 days. Therefore, and in the absence of body weight effects, these observations were assessed as borderline effects of a marginal systemic toxicity of clindamycin at $200 \mathrm{mg} / \mathrm{kg}$ body weight. Relative changes in body weight and food consumption noted upon administration of the test compounds are shown in Table 1. Treated animals did not present any significant changes with respect to body weight when compared to the controls.

Table 1 Relative changes in body weight and food consumption of male and female $\mathrm{Crl}: \mathrm{Wi}(\mathrm{Han})$ rats $(\mathrm{N}=5$ per group) dosed for 28 days compared to the diet controls. Data were collected on study days 6, 13 and 27 . Treated animals did not present any significant changes $(\mathrm{p}<0.05)$ in body weight compared to the control groups $(\mathrm{N}=10$ per group).

\begin{tabular}{lccccc}
\hline \multirow{2}{*}{ Compound } & \multirow{2}{*}{ Day } & \multicolumn{2}{c}{ Body weight } & \multicolumn{2}{c}{ Food consumption } \\
& & $\mathbf{~ m}$ & $\mathbf{f}$ & $\mathbf{~ m}$ & $\mathbf{f}$ \\
\hline Clindamycin & 6 & 0.97 & 0.99 & 1.30 & 0.80 \\
\cline { 2 - 6 } & 13 & 0.95 & 0.98 & 1.12 & 1.19 \\
\cline { 2 - 6 } & 27 & 0.94 & 1.00 & 0.99 & 1.16 \\
\hline Lincomycin & 6 & 1.00 & 0.99 & 1.00 & 0.70 \\
\cline { 2 - 6 } & 13 & 0.98 & 1.00 & 1.03 & 0.99 \\
\hline $0.5 \%$ CMC & 27 & 0.96 & 1.05 & 1.18 & 0.97 \\
& 6 & 1.00 & 0.99 & 0.98 & 1.00 \\
\hline & 13 & 0.98 & 1.01 & 0.99 & 0.90 \\
\hline
\end{tabular}




\section{Metabolite Profiling in plasma and feces}

Table 2 shows the percentage of altered metabolites of the metabolome analysis in plasma and feces of antibiotic-treated rats as well as the animals receiving $0.5 \% \mathrm{CMC}$ compared to the diet control animals.

Clindamycin treatment showed a significant effect on the plasma metabolite profiles in all the treated animals compared to the controls (see Table 2). Male animals exhibited a percentage of changed metabolites of $32 \%$ (day 7 ) and $28 \%$ at the end of the administration period (day 28). More down- than upregulated metabolites were observed. Females presented a balanced ratio of down- and upregulated metabolites on day 7 and day 28 . At day $7,25 \%$ of the total measured metabolites were altered, on day 14 , the total changes increased to $34 \%$ due to a rise in upregulated metabolites. On the last day of treatment, the total amount of altered metabolites was $24 \%$.

The total amount of metabolite changes observed with lincomycin treatment was similar compared to clindamycin (see Table 2). The number of total significantly altered metabolites in males dropped from $32 \%$ on day 7 to $22 \%$ at the end of the study (day 28 ). In the female group, the percentage of changed metabolites also decreased from day 7 $(36 \%)$ to day $28(28 \%)$. In general, in both sexes, the number of downregulated metabolites in plasma was higher than the number of upregulated metabolites. Females showed slightly higher percentage of total changes compared to the controls than male animals, especially on day 7 .

In the CMC group of male animals the percentage of changed metabolites fluctuated strongly over time and decreased from $32 \%$ to $19 \%$ compared to the diet control showing more upregulated than downregulated metabolites. Due to these inconsistent fluctuations in the plasma metabolites, the overall profile strength was evaluated to enable an assessment whether an effect is significant or in the range of normal biological fluctuations of the metabolome (see Table 3).

Table 2 displays the percentages of changed metabolites in the feces of the treated animals compared to the diet controls on day 28. The values show that the antibiotics administered had a prominent impact on the feces metabolome. Around half of the total measured metabolites were significantly altered after lincomycin treatment in both males and females. Regarding clindamycin, males treated with this antibiotic exhibited 57\% changed metabolites. Females expressed with $44 \%$ a lower number of total changes. In general, after lincosamides treatment the number of significantly upregulated metabolites in feces was at least three times higher than the downregulated ones.

After CMC treatment the feces metabolome just showed only very minor changes (4-5\%) compared to the diet control. 
Table 2 Statistically significantly downregulated (blue) and upregulated (red) measured metabolites compared to the diet controls in plasma (day 7, 14 and 28, p < 0.2) and feces (day 28, p < 0.05) of male and female Wistar rats after antibiotic treatment with clindamycin, lincomycin and $0.5 \% \mathrm{CMC}$ for 28 days.

\begin{tabular}{|c|c|c|c|c|c|c|c|c|}
\hline & \multicolumn{4}{|c|}{ Clindamycin, males } & \multicolumn{4}{|c|}{ Clindamycin, females } \\
\hline & \multicolumn{3}{|c|}{ Plasma } & \multirow{2}{*}{$\begin{array}{l}\text { Feces } \\
28 \mathrm{~d}\end{array}$} & \multicolumn{3}{|c|}{ Plasma } & \multirow{2}{*}{$\begin{array}{l}\text { Feces } \\
28 d\end{array}$} \\
\hline & $7 \mathrm{~d}$ & $14 \mathrm{~d}$ & $28 \mathrm{~d}$ & & $7 \mathrm{~d}$ & $14 \mathrm{~d}$ & $28 \mathrm{~d}$ & \\
\hline Total metabolites & 212 & 212 & 211 & 182 & 219 & 218 & 218 & 180 \\
\hline$\%$ down & 24.5 & 16.0 & 16.1 & 14.3 & 12.8 & 12.8 & 12.8 & 11.7 \\
\hline$\%$ up & 7.5 & 9.0 & 12.3 & 42.9 & 12.3 & 21.6 & 11.0 & 32.8 \\
\hline$\%$ total & 32.1 & 25.0 & 28.4 & 57.1 & 25.1 & 34.4 & 23.9 & 44.4 \\
\hline
\end{tabular}

\begin{tabular}{|c|c|c|c|c|c|c|c|c|}
\hline & \multicolumn{4}{|c|}{ Lincomycin, males } & \multicolumn{4}{|c|}{ Lincomycin, females } \\
\hline & \multicolumn{3}{|c|}{ Plasma } & \multirow{2}{*}{$\begin{array}{l}\text { Feces } \\
28 \mathrm{~d}\end{array}$} & \multicolumn{3}{|c|}{ Plasma } & \multirow{2}{*}{$\begin{array}{l}\text { Feces } \\
28 \mathrm{~d}\end{array}$} \\
\hline & $7 d$ & $14 \mathrm{~d}$ & $28 \mathrm{~d}$ & & $7 d$ & $14 \mathrm{~d}$ & $28 \mathrm{~d}$ & \\
\hline Total metabolites & 214 & 212 & 214 & 183 & 219 & 216 & 217 & 180 \\
\hline$\%$ down & 21.5 & 26.4 & 16.8 & 8.2 & 22.8 & 19.0 & 14.7 & 9.4 \\
\hline$\%$ up & 10.3 & 6.1 & 5.1 & 43.7 & 13.2 & 11.6 & 12.9 & 40.6 \\
\hline \multirow[t]{4}{*}{$\%$ total } & 31.8 & 32.5 & 22.0 & 51.9 & 36.1 & 30.6 & 27.6 & 50.0 \\
\hline & \multicolumn{4}{|c|}{$0.5 \%$ CMC, males } & \multicolumn{4}{|c|}{$0.5 \%$ CMC, females } \\
\hline & \multicolumn{3}{|c|}{ Plasma } & \multirow{2}{*}{$\begin{array}{l}\text { Feces } \\
28 d\end{array}$} & \multicolumn{3}{|c|}{ Plasma } & Feces \\
\hline & $7 d$ & $14 d$ & $28 \mathrm{~d}$ & & $7 d$ & $14 \mathrm{~d}$ & $28 \mathrm{~d}$ & $28 \mathrm{~d}$ \\
\hline Total metabolites & 218 & 218 & 219 & 200 & 212 & 212 & 219 & 199 \\
\hline$\%$ down & 12.4 & 12.8 & 5.0 & 1.0 & 12.7 & 5.7 & 19.6 & 3.5 \\
\hline$\%$ up & 19.3 & 16.1 & 14.2 & 3.5 & 9.0 & 12.7 & 8.7 & 0.5 \\
\hline$\%$ total & 31.7 & 28.9 & 19.2 & 4.5 & 21.7 & 18.4 & 28.3 & 4.0 \\
\hline
\end{tabular}

A Principle Component Analysis (PCA) was conducted to visualize treatment-related effects on the feces metabolites. The PCA demonstrated that CMC treatment clusters with the control animals and after 4 weeks of lincosamides administration a clear treatmentrelated effect could be observed in the feces metabolome (see Figure 2). The main separation was driven by the treatment-related effect. The female group showed a separation of clindamycin and lincomycin in principal component 2 . Taken together, these analyses show that the observed alterations of the metabolites in the feces is mainly driven by the treatment effect followed by the specific antibiotic applied (either lincomycin or clindamycin). 
a)

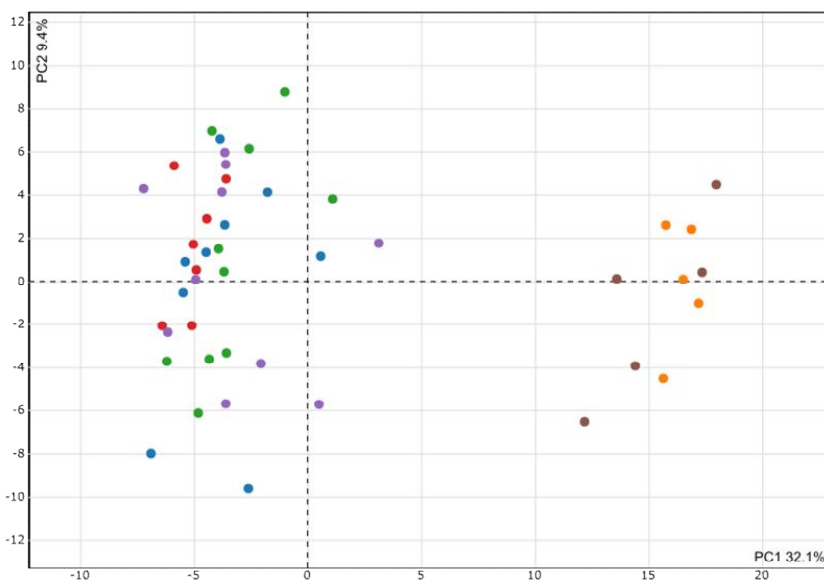

b)

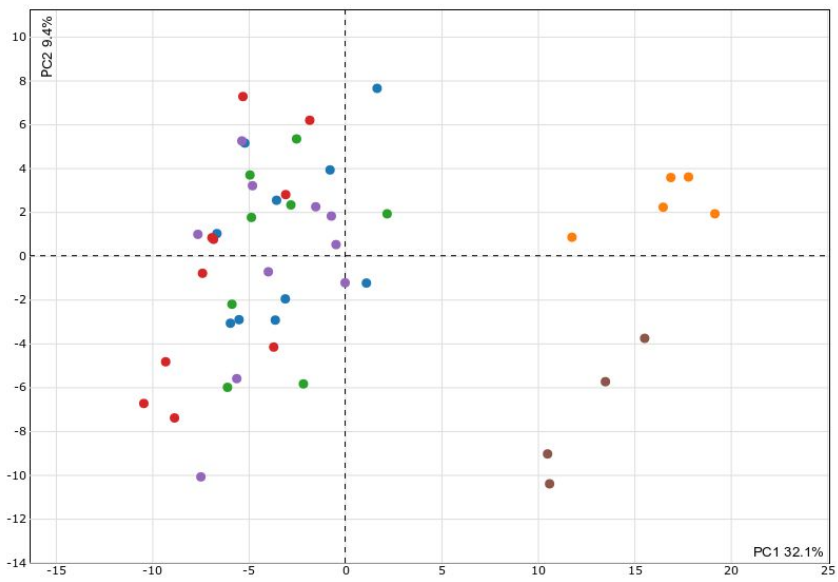

Treatment Group

Study control of $\mathrm{CMC}$

Study control of lincomycin

Study control of clindamycin

$0.5 \% \mathrm{CMC}$

Lincomycin

Clindamycin

Figure 2 Treatment effect of clindamycin and lincomycin on the feces metabolome of male animals (a) and female animals (b). PCAs of fecal metabolic profiles of male $(\mathrm{N}=29)$ and female $(\mathrm{N}=28)$ control animals, male and female animals dosed with clindamycin $(200 \mathrm{mg} / \mathrm{kg}$ bw/d) and lincomycin $(300 \mathrm{mg} / \mathrm{kg} \mathrm{bw} / \mathrm{d})(\mathrm{N}=5$ per group, except female group of clindamycin with $\mathrm{N}=4)$ and animals receiving $0.5 \%$ CMC $(\mathrm{N}=10$ for females, $\mathrm{N}=7$ for males) for 28 days. Feces samples were taken on study 28 after overnight fasting.

The profile strength of each metabolic profile was calculated in the MetaMap®Tox database (see Table 3) to evaluate the magnitude of the changes which reflects the impact of a treatment on the metabolome compared to the diet control of the study. In the feces, lincosamides treatment showed both in males and females a profound effect (lincomycin with 3.54 for females and 4.05 for males, and clindamycin with 3.80 for females and 3.96 for males) whereas the profile strength of CMC treatment was below 1.0. The profile strength of the plasma metabolome in both sexes was for lincosamides treatment 0.9-1.0. For CMC treatment the profile strength was in the range of other control groups in the database ( 0.85 for males and 0.7 for females). 
Table 3 Profile strength of the metabolite profile in plasma and feces of male and female Wistar rats after treatment with clindamycin, lincomycin and $0.5 \% \mathrm{CMC}$.

\begin{tabular}{lcccccc}
\hline & \multicolumn{2}{c}{ Clindamycin } & \multicolumn{2}{c}{ Lincomycin } & \multicolumn{2}{c}{$\mathbf{0 . 5 \%}$ CMC } \\
& males & females & males & females & males & females \\
\hline Plasma & 1.06 & 0.93 & 0.97 & 1.02 & 0.85 & 0.70 \\
Feces & 3.96 & 3.80 & 4.05 & 3.54 & 0.83 & 0.87 \\
\hline
\end{tabular}

\section{Specific plasma metabolite patterns of lincosamides}

Numerous studies have demonstrated that the gut microbiota influences the host metabolic phenotype. The microorganisms present in the intestine produce or modify different metabolites. These metabolites can be (re-)absorbed into the system and consequently can be detected in the plasma. Thus, in the present study, specific plasma metabolome patterns were established to characterize the effects of the lincosamides antibiotics on the functionality of the microbiome. Therefore, each metabolite value of a treated group was evaluated against the value of the diet control of the corresponding study and expressed as a relative (fold-change) value of the control.

\section{Plasma metabolite pattern in male animals}

A plasma pattern for the effect of lincosamides on the male microbiome was established and validated in MetaMap ${ }^{\circledR T o x}$ (see Figure 3). This pattern consists in a total of 12 plasma altered metabolites. Ten of these metabolites were significantly decreased and two were increased (i.e. 3-hydroxybutyrate and taurocholic acid) after the antibiotics administration compared to the control group. Noteworthy, 9 of the 12 downregulated metabolites were complex lipids, fatty acids and related metabolites.

Significant changes were also seen in amino acids and energy metabolism. At all three time points (day 7, 14, 28) and for both lincosamides tested, cholic acid and glycochenodeoxycholic acid in plasma were profoundly decreased. In contrast, taurocholic acid presented highly elevated levels. The highest relative abundance in plasma was reached on day 7 where the values for lincomycin showed a 7.82 -fold and 4.40 -fold increase compared to the controls, respectively. On day 28 , the relative abundance in plasma for taurocholic acid dropped to 4.22 (lincomycin) and 2.60 (clindamycin). Another significantly increased metabolite noted after lincosamides treatment was 3hydroxybutyrate, with concentrations in plasma increased towards the end of the administration period: 1.31 after clindamycin and 1.19 after lincomycin treatment. Other metabolites belonging to the class of complex lipids, fatty acids and related, such as arachidonic acid, heptadecanoic acid and diacylglycerol (DAG), were shown to be significantly decreased at most time points. 
Impact of lincosamides on the bacterial composition and metabolite profile

\begin{tabular}{|c|c|c|c|c|c|c|c|c|}
\hline \multirow[t]{2}{*}{ Metabolite } & \multirow[t]{2}{*}{ Direction } & \multicolumn{3}{|c|}{ Clindamycin } & \multicolumn{3}{|c|}{ Lincomycin } & \multirow[t]{2}{*}{ Metabolite class } \\
\hline & & $7 \mathrm{~d}$ & $14 \mathrm{~d}$ & $28 \mathrm{~d}$ & $7 \mathrm{~d}$ & $14 \mathrm{~d}$ & $28 \mathrm{~d}$ & \\
\hline Tyrosine & $\downarrow$ & 0.81 & 0.75 & 0.90 & 0.82 & 0.84 & 0.93 & Amino acids \\
\hline Glycine & $\downarrow$ & 0.76 & 0.81 & 0.88 & 0.62 & 0.62 & 0.92 & \\
\hline Taurocholic acid & $\uparrow$ & 4.40 & 3.12 & 2.60 & 7.82 & 6.36 & 4.22 & Complex lipids, fatty acids and related \\
\hline Glycochenodeoxycholic acid & $\downarrow$ & 0.00 & 0.01 & NA & 0.00 & 0.01 & 0.03 & metabolites \\
\hline Cholic acid & $\downarrow$ & 0.00 & 0.04 & 0.00 & 0.00 & 0.00 & 0.01 & \\
\hline DAG (C18:1,C18:2) & $\downarrow$ & 0.80 & 0.92 & 0.81 & 0.72 & 0.69 & 0.93 & \\
\hline Arachidonic acid (C20:cis $[5,8$, & $\downarrow$ & 0.73 & 0.80 & 0.96 & 0.75 & 0.74 & 0.97 & \\
\hline Heptadecanoic acid (C17:0) & $\downarrow$ & 0.82 & 0.78 & 0.76 & 1.00 & 0.74 & 0.79 & \\
\hline Lysophosphatidylcholine (C20 & $\downarrow$ & 0.97 & 0.83 & 0.85 & 0.91 & 0.86 & 1.04 & \\
\hline Lysophosphatidylcholine (C17 & $\downarrow$ & 0.86 & 0.89 & 0.83 & 0.99 & 0.89 & 0.93 & \\
\hline Unknown metabolite & $\downarrow$ & 0.83 & 0.87 & 0.92 & 0.96 & 0.94 & 0.91 & \\
\hline 3-Hydroxybutyrate & $\uparrow$ & 1.18 & 1.50 & 1.31 & 1.06 & 1.09 & 1.19 & Energy metabolism and related metabolites \\
\hline
\end{tabular}

Figure 3 Heatmap of statistically significantly increased and decreased values of metabolite changes after treatment with lincosamides of male animals (Welch $t$ test; $p<0.2$ ). Metabolite concentration changes relative to the diet controls of each study $(\mathrm{N}=10$ per group) in plasma of male $\mathrm{Crl} / \mathrm{Wi}(\mathrm{Han})$ rats $(\mathrm{N}=5$ per group) dosed with lincomycin $(300 \mathrm{mg} / \mathrm{kg}$ bw/d) or clindamycin $(200 \mathrm{mg} / \mathrm{kg}$ bw/d) for 28 days. Blood samples were taken on study days 7, 14 and 28 after overnight fasting: figures in red boxes are significantly increased and those in blue boxes significantly decreased metabolite value changes. The intensity of the color corresponds to the magnitude of the fold change. "NA" indicates that for this analyte, treatment and/or analysis groups there are no contrasts available.

\section{Plasma metabolite pattern in female animals}

A plasma metabolome pattern was also created for the female animals treated with lincosamides. This pattern contains ten metabolites that showed to be altered after the antibiotics treatment (see Figure 4). Six metabolites were significantly decreased and four significantly increased compared to the diet control group. Changes were seen in amino acids and related metabolites, carbohydrates and related metabolites, complex lipids, fatty acids and related metabolites, energy metabolism and related and in miscellaneous metabolites. As in the male pattern, cholic acid and glycochenodeoxycholic acid were remarkably decreased at all the time points (day $7,14,28$ ). The relative abundance in plasma ranged from 0.02 on day 7 to 0.01 on day 28 (clindamycin) and from undetectable concentrations on day 14 to 0.06 on the last day of the study (lincomycin). Also, similar to the male pattern, the values of taurocholic acid and 2-hydroxybutyrate were significantly increased. Particularly after lincomycin treatment, taurocholic acid was highly upregulated exhibiting its highest value at day 7 (4.12). However, at the last day of the study the relative plasma concentrations of taurocholic were similar for both antibiotics; 1.83 (lincomycin) and 1.84 (clindamycin). 2-Hydroxybutyrate was 3.80-fold increased in plasma after clindamycin administration, dropping on day 28 to an 1.93 -fold increase. Lincomycin treatment caused a significant increase of 2-hydroxybutyrate on day 7 (1.23) and day 14 (1.57). Regarding the amino acids and related metabolites, the female group showed a significant increase in glutamate during days 7 and 14. Additionally, low relative abundance in plasma was found for 3-hydroxyindole, ranging from 0.06 on day 7 to 0.33 on day 28 (lincomycin) and from 0.46 on day 7 to 0.17 on day 14 . High levels of glucuronic acid were also observed after treatment with both antibiotics. 17-hydroxypregnenolone, a steroid hormone, was found to be strongly decreased after clindamycin administration and dropped to undetectable levels after lincomycin treatment. Besides, the concentrations of 
hippuric acid were clearly decreased with both substances over all the times points, except for day 28 of lincomycin, where this metabolite was singularly upregulated. Relative plasma values of hippuric acid ranged from 0.07 at day 7 to 0.12 on day 28 (clindamycin) and from 0.33 at day 7 to 0.75 on day 14 with a followed increased to 1.96 at day 28 (lincomycin).

\begin{tabular}{|c|c|c|c|c|c|c|c|c|}
\hline \multirow[t]{2}{*}{ Metabolite } & \multirow[t]{2}{*}{ Direction } & \multicolumn{3}{|c|}{ Clindamycin } & \multicolumn{3}{|c|}{ Lincomycin } & \multirow[t]{2}{*}{ Metabolite class } \\
\hline & & $7 \mathrm{~d}$ & $14 \mathrm{~d}$ & $28 \mathrm{~d}$ & $7 \mathrm{~d}$ & $14 \mathrm{~d}$ & $28 \mathrm{~d}$ & \\
\hline Glycine & $\downarrow$ & 0.78 & 0.85 & 0.91 & 0.64 & 0.68 & 1.01 & \multirow[t]{2}{*}{ Amino acids } \\
\hline Glutamate & $\uparrow$ & 1.23 & 1.41 & 1.04 & 1.31 & 1.21 & 1.15 & \\
\hline 3-Hydroxyindole & $\downarrow$ & 0.46 & 0.17 & 0.09 & 0.06 & 0.04 & 0.33 & Amino acids related metabolites \\
\hline Glucuronic acid & $\uparrow$ & 1.55 & 1.51 & 1.35 & 1.59 & 1.39 & 1.72 & Carbohydrates and related metabolites \\
\hline Taurocholic acid & $\uparrow$ & 1.44 & 1.55 & 1.84 & 4.12 & 3.29 & 1.83 & \multirow{3}{*}{$\begin{array}{l}\text { Complex lipids, fatty acids and related } \\
\text { metabolites }\end{array}$} \\
\hline Glycochenodeoxycholic acid & $\downarrow$ & 0.07 & 0.11 & 0.21 & 0.16 & 0.04 & 0.18 & \\
\hline Cholic acid & $\downarrow$ & 0.02 & 0.03 & 0.01 & 0.01 & 0.00 & 0.06 & \\
\hline 2-Hydroxybutyrate & $\uparrow$ & 2.28 & 3.08 & 1.93 & 1.23 & 1.57 & 1.04 & Energy metabolism and related metabolites \\
\hline 17-Hydroxypregnenolone & $\downarrow$ & 0.01 & 0.11 & 0.08 & 0.00 & 0.00 & 0.00 & Hormones \\
\hline Hippuric acid & $\downarrow$ & 0.07 & 0.13 & 0.12 & 0.33 & 0.75 & 1.96 & Miscellaneous \\
\hline
\end{tabular}

Figure 4 Heatmap of statistically significantly increased and decreased values of metabolite changes after treatment with lincosamides of female animals (Welch $t$ test; $p<0.2$ ). Metabolite concentration changes relative to the diet controls of each study $(\mathrm{N}=10$ per group) in plasma of female $\mathrm{Crl} / \mathrm{Wi}(\mathrm{Han})$ rats $(\mathrm{N}=5$ per group) dosed with lincomycin $(300 \mathrm{mg} / \mathrm{kg}$ bw/d) or clindamycin $(200 \mathrm{mg} / \mathrm{kg} \mathrm{bw} / \mathrm{d})$ for 28 days. Blood samples were taken on study days 7, 14 and 28 after overnight fasting: figures in red boxes are significantly increased and those in blue boxes significantly decreased metabolite value changes compared to controls. The intensity of the color corresponds to the magnitude of the fold change.

\section{Significant altered metabolites in feces and plasma}

Table 4 shows an overall comparison of significant altered metabolites after lincosamides treatment where significant increased and decreased plasma metabolites were compared with the changes in feces. Feces metabolites that were found to be altered more than 5 -fold were also included in the table. 
Table 4 Overall comparison of significant altered metabolites after lincosamides treatment. Statistically significant increased and decreased plasma metabolites were compared with the changes in feces. Additionally, feces metabolites that were found altered more than 5-fold were included in the table and marked with *. Arrows indicate the direction of regulation. "NA" indicates that for this analyte, treatment and/or analysis groups there are no contrasts available.

\begin{tabular}{|c|c|c|c|c|c|}
\hline \multirow[t]{2}{*}{ Metabolite } & \multirow[t]{2}{*}{ Class } & \multicolumn{2}{|c|}{ Plasme } & \multicolumn{2}{|c|}{ Feces } \\
\hline & & 0 & 우 & $0^{2}$ & q \\
\hline Glutamate & Amino acids & & $\bar{\uparrow}$ & & $\bar{\uparrow}$ \\
\hline Glycine & and related & $\downarrow$ & $\downarrow$ & $\uparrow$ & $\uparrow$ \\
\hline Taurine & metabolites & & & $\uparrow^{*}$ & $\uparrow^{*}$ \\
\hline Tryptophan & & & & $\uparrow^{*}$ & $\uparrow^{*}$ \\
\hline Tyrosine & & $\downarrow$ & & $\uparrow^{*}$ & $\uparrow^{*}$ \\
\hline 3-Hydroxyindole & & & $\downarrow$ & & NA \\
\hline Indole-3-lactic acid & & & & $\uparrow^{*}$ & \\
\hline Kynurenic acid & & & & $\uparrow^{*}$ & $\uparrow^{*}$ \\
\hline Creatine & & & & $\uparrow^{*}$ & $\uparrow^{*}$ \\
\hline Creatinine & & & & $\uparrow^{*}$ & $\uparrow^{*}$ \\
\hline Hippuric acid & & & $\downarrow$ & & NA \\
\hline Cholic acid & Bile acids & $\downarrow$ & $\downarrow$ & $\uparrow^{*}$ & $\uparrow^{*}$ \\
\hline Glycochenodeoxycholic acid & & $\downarrow$ & $\downarrow$ & & $\downarrow^{*}$ \\
\hline Taurocholic acid & & $\uparrow$ & $\uparrow$ & $\uparrow^{*}$ & $\uparrow^{*}$ \\
\hline 4-Hydroxysphinganine (t18:0, Phytosphingosine) & Complex lipids, & & & $\uparrow^{*}$ & $\uparrow^{*}$ \\
\hline Arachidonic acid $(\mathrm{C} 20: \operatorname{cis}[5,8,11,14] 4)$ & fatty acids & $\downarrow$ & & & \\
\hline DAG (C18:1,C18:2) & and related & $\downarrow$ & & $\uparrow$ & $\uparrow$ \\
\hline Heptadecanoic acid (C17:0) & & $\downarrow$ & & $\downarrow$ & $\downarrow$ \\
\hline Hexadecanol & & & & $\downarrow^{*}$ & $\downarrow^{*}$ \\
\hline Linoleic acid (C18:cis[9,12]2) & & & & & $\uparrow^{*}$ \\
\hline Lysophosphatidylcholine (C17:0) & & $\downarrow$ & & & \\
\hline Lysophosphatidylcholine (C20:4) & & $\downarrow$ & & & \\
\hline TAG (C16:0,C18:2) & & & & $\uparrow^{*}$ & $\uparrow^{*}$ \\
\hline TAG (C18:1,C18:2) & & & & $\uparrow^{*}$ & $\uparrow^{*}$ \\
\hline TAG (C18:2,C18:2) & & & & $\uparrow^{*}$ & $\uparrow^{*}$ \\
\hline TAG (C16:0,C18:1,C18:3) & & & & $\uparrow^{*}$ & $\uparrow^{*}$ \\
\hline TAG (C18:1,C18:2,C18:3) & & & & $\uparrow^{*}$ & $\uparrow^{*}$ \\
\hline Unknown metabolite I & & $\downarrow$ & & & \\
\hline Unknown metabolite II & & & & $\uparrow^{*}$ & $\uparrow^{*}$ \\
\hline Glucuronic acid & Others & & $\uparrow$ & $\uparrow$ & $\uparrow$ \\
\hline Xylitol & & & & $\uparrow^{*}$ & $\uparrow^{*}$ \\
\hline 3-Hydroxybutyrate & & $\uparrow$ & & $\uparrow^{*}$ & $\uparrow^{*}$ \\
\hline 2-Hydroxybutyrate & & & $\uparrow$ & $\uparrow^{*}$ & $\uparrow^{*}$ \\
\hline 17-Hydroxypregnenolone & & & $\downarrow$ & $\mathrm{N} A$ & NA \\
\hline
\end{tabular}




\section{Community analysis of the gut microbiome}

To characterize the changes in the gut microbiota composition after lincosamides administration, 16S ribosomal RNA gene sequencing was performed. DNA was extracted from feces samples collected at the last day of the study (day 28) from the control and treated animals, and an analysis of bacterial taxonomic units was carried out.

A clear treatment-related effect for both antibiotics on the gut microbiome was revealed by a principal coordinate analysis (PCoA) of weighted UniFrac distances (see Figure 5). The main separation on Axis 1 was driven by the treatment. Community structures of treated animals, both male and female, clustered together and were clearly separated from the cluster of the community composition of the control groups, which clustered with the CMC treated animals.

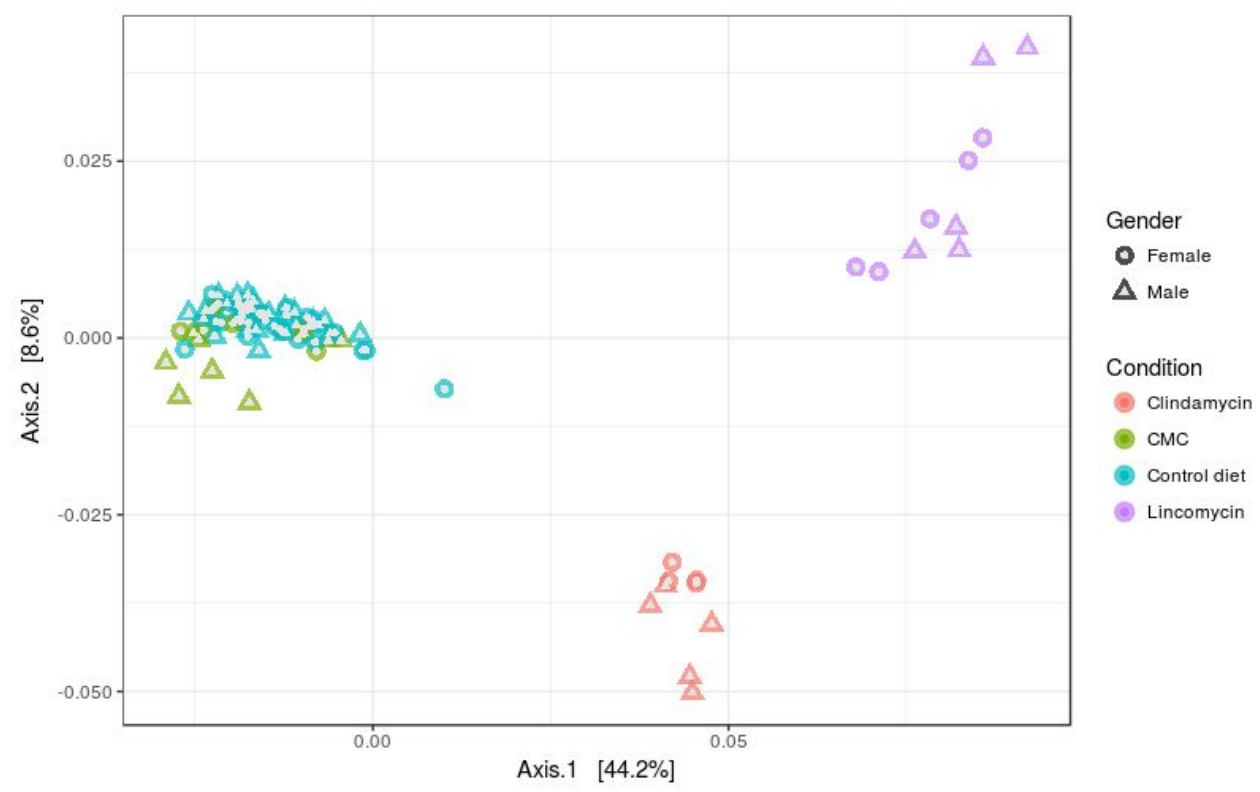

Figure 5 Principal coordinate analysis $(\mathrm{PCoA})$ of the gut microbial community showing a treatment-related effect of clindamycin and lincomycin versus the vehicle carboxymethylcellulose (CMC, 0.5\%) and control groups in the gut microbial composition of male and female animals. Feces samples were taken on day 28 of the study, DNA was extracted and the 16S ribosomal RNA gene was sequenced. PCoA was calculated using weighted UniFrac distance on the denoised dataset obtained from the dada2 workflow.

To dig deeper into the changes observed in the PCoA, an analysis of alpha and beta diversity was performed. To assess alpha diversity, effective species number was calculated for each sample by taking the exponential of Shannon-Weiner index (Chao et al., 2010). Effective species is a standardized diversity unit that represents the amount of diversity in a population of evenly distributed species. Effective species number for each sample was visualized and Kruskal-Wallis statistics were calculated using the diversity plugin of QIIME2 (see Supplementary Material). Analysis of effective species number by treatment 
showed that despite appearing similar on the PCoA, CMC seemed to slightly increase the diversity from around 80 effective species to 100 , however this change was not significant after testing using Kruskal-Wallis. The diversity decline in antibiotic treated samples was similar for both antibiotics very pronounced; decreasing the effective species to fewer than 20 (Kruskal-Wallis, $\mathrm{q}<0.01$ ).

For beta diversity, a PERMANOVA analysis (Anderson, 2017) of weighted UniFrac distance was performed using the diversity plugin in QIIME2 to reveal that CMC had no effect on the females' microbiome $(\mathrm{F}=1.3, \mathrm{p}=0.182)$ and just a minor, yet significant, effect on the males' microbiome $(\mathrm{F}=1.9, \mathrm{p}=0.036)$. However, the microbial composition was substantially changed for both males and females upon treatment with lincomycin $(\mathrm{F}>$ 53.9, $\mathrm{p}<0.002)$ or clindamycin $(\mathrm{F}>46.5, \mathrm{p}<0.003)$ as compared to the controls (see Suplementary Material). Both antibiotics demonstrated similar drastic alterations of the gut microbiome at the phylum level (see Figure 6). The microbial community of control animals was composed mainly by Firmicutes ( 45-48\%) and Bacteroidetes ( 40-44\%), followed by Verrucomicrobia (7-9\%), unidentified bacteria ( 3\%), Proteobacteria $(\sim 0.8 \%)$, Tenericutes $(\sim 0.2 \%)$ and Deferribacteres ( $\sim 0.02-0.04 \%)$. After 28 days of clindamycin and lincomycin administration the gut bacterial community was composed by a high percentage of Firmicutes (more than $90 \%$ in lincomycin and $80 \%$ in clindamycin) followed by a low relative proportion of Proteobacteria ( $3 \%$ lincomycin, $15 \%$ clindamycin) and other unidentified phyla (1.5-2\% lincomycin, 3-4\% clindamycin). Bacteroidetes $(<0.015 \%)$ and Verrucomicrobia $(<0.1 \%)$ were drastically reduced. Deferribacteres and Tenericutes were completely wiped out in the antibiotic-treated animals, while the proportions of Firmicutes proportions were twice as compared to the control groups. Additionally, the levels of Proteobacteria were greatly increased in the treated groups.

(a)

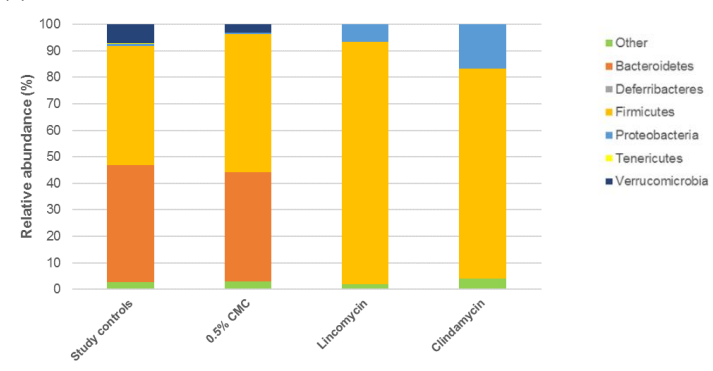

(b)

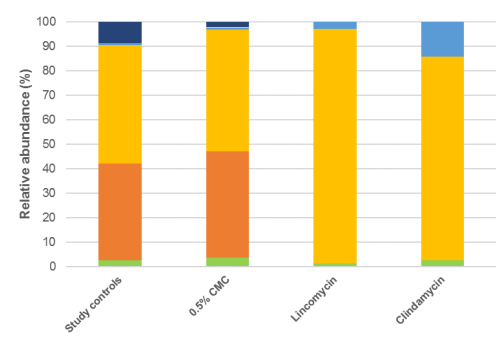

Figure 6 Relative abundance of gut microbes at phylum level of control animals $(\mathrm{N}=30$ males and $\mathrm{N}=28$ females) and after $0.5 \% \mathrm{CMC}(\mathrm{N}=9$ males and $\mathrm{N}=10$ females $)$, clindamycin and lincomycin treatment $(\mathrm{N}=5$ per group and sex, except female group of clindamycin with $\mathrm{N}=4$ ) in male (a) and female animals (b). Feces samples were taken on day 28 of the study. Male and female $\mathrm{Crl}: \mathrm{Wi}(\mathrm{Han})$ rats were dosed with $0.5 \% \mathrm{CMC}$, lincomycin $(300 \mathrm{mg} / \mathrm{kg}$ bw/d) and clindamycin $(200 \mathrm{mg} / \mathrm{kg} \mathrm{bw} / \mathrm{d})$, respectively, for 28 days. 
The CMC group showed slight differences in composition between male and female animals which could also be observed in the PCoA. (see supplementary material). In male animals there was a slight decrease of Bacteroidetes $(\sim 3 \%)$, Proteobacteria, Tenericutes, Verrucomicrobia and an increase of Firmicutes ( $\sim 7 \%)$. In comparison, the female animals just showed a slight decrease of Verrucomicrobia $(\sim 6 \%)$ and an increase of $\sim 4 \%$ of Bacteroidetes. Taken together, these results demonstrate an evident effect of clindamycin and lincomycin on the gut microbial diversity and taxa proportions but no or just minor changes after CMC treatment.

For a deeper analysis, pairwise Krona plots were established to show the differences between groups with a color code from 1 (pink) meaning an increase after treatment; to -1 (green) meaning a decrease after treatment. More information is available in the materials and methods section. Since the PCoA of the microbial gut community showed no obvious differences between male and female animals, the pairwise Krona plots were established with the combined data of male and female animals (see Figure 7). After CMC treatment, the male group showed a decrease in the bacteria of the order Rhodospirillales (family unassigned). In both male and female animals, lincosamides treatment lead to an increase or decrease, respectively, of 10 to 16 different bacterial families mainly deriving from Firmicutes and Bacteroidetes (see Supplementary Material). 


\section{a) Control vs $0.5 \% \mathrm{CMC}$}

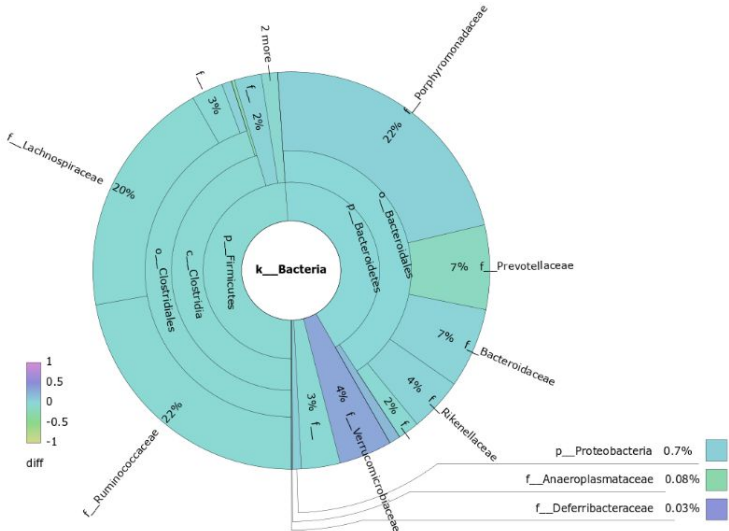

c) Control vs lincomycin

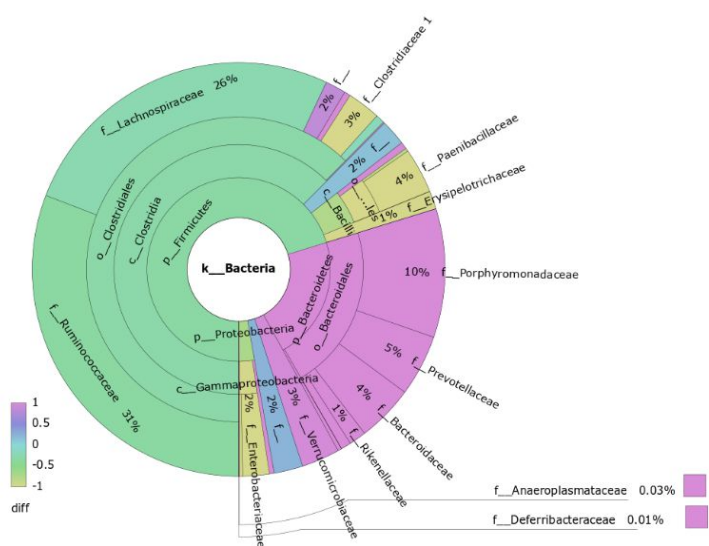

\section{b) Control vs clindamycin}

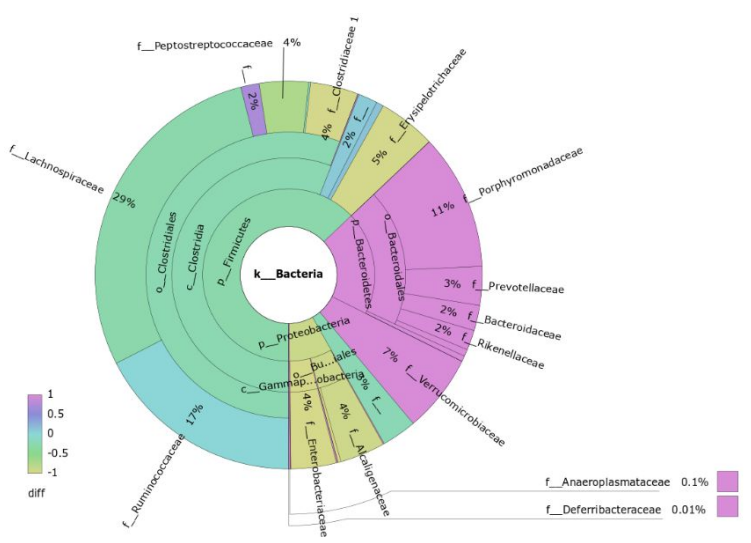

Figure 7 Pairwise Krona plots showing the difference (diff) of the microbial composition up to family level after $0.5 \%$ CMC (a), clindamycin (b) and lincomycin (c) treatment. The percentage is the average of each family over control and treatment. When taxa contributions are the same, the difference is 0. Pink color means a decrease, yellow an increase of the bacterial family compared to the bacterial composition of the control animals. Feces samples were taken on day 28 of the study, DNA was extracted and the 16S ribosomal RNA gene was sequenced. Core diversity analysis was obtained by denoising the raw reads using dada2 workflow. Male and female $\mathrm{Crl}: \mathrm{Wi}(\mathrm{Han})$ rats $(\mathrm{N}=5$ per group) were dosed with $0.5 \% \mathrm{CMC}$, lincomycin $(300 \mathrm{mg} / \mathrm{kg}$ bw/d) and clindamycin (200 mg/kg bw/d), respectively, for 28 days. 


\section{Discussion}

Microorganisms present in the gastrointestinal tract have the capacity to synthesize or modify different metabolites. These metabolites can be taken up into the host system and consequently can be detected in plasma. However, even though the effects of the microorganisms in the host metabolic phenotype is recognized, little is known about the mechanisms and consequences of these host-microbe interactions.

In the present study, a shift of the microbiome was induced by the administration of two antibiotics, i.e. lincomycin and clindamycin, which belong to the class of lincosamides. Lincosamides were chosen due to their broad-spectrum activity against microbes and their low systemic toxicity in the host. Several studies have demonstrated that lincosamides do not induce organ/systemic toxicity even at high doses in long-term toxicity studies (Gray et al., 1972, 1971, 1966, 1964). In our study, treated animals showed no significant alteration in food consumption and body weight compared to control animals. Clinical symptoms, in particular salivation, found after the administration of clindamycin were probably an acute response to the administration treatment (gavage, potential bad taste, slight irritant effects) rather than a form of systemic toxicity identifiable by metabolomics. The temporarily observed clinical findings (semi-closed eyelid, piloerection and general poor condition) on maximum of 3 days during the administration period of 28 days were assessed as borderline effects of slight systemic toxicity, which are in the absence of any body weight effect unlikely identifiable by metabolomics. For these reasons, it can be assumed that the plasma metabolome changes caused by these antibiotics were the result of their effect on the microbial functionality.

$\mathrm{CMC}$, which is widely used in toxicity studies as a vehicle, has been reported to have an effect on the microbiome and is suggested to be associated with the development of inflammatory bowel disease (IBD) (Chassaing et al., 2015; Martino et al., 2017). Therefore, a separate group for each sex received $0.5 \% \mathrm{CMC}$ to identify potential significant shifts in microbiome communities as well as in the metabolome. CMC treatment showed no effect on the fecal microbiome composition and virtually no effect on the fecal metabolome. Moreover, the plasma pattern strength, $0.7-0.85$, is in the range of control values. Given these facts it is concluded that $\mathrm{CMC}$ has no relevant biological effect on the microbiome nor on the fecal and plasma metabolome.

The profile strength, which reflects the impact of a treatment on the overall metabolome regarding the magnitude of change, showed that in plasma the lincosamides treatment had no strong effect regarding the overall metabolite profile. However, minor changes in tightly controlled metabolites or just few metabolites that are highly up- or downregulated, are not reflected in this evaluation. Though, they are detected in the process of establishing the specific plasma metabolome patterns. Even though the profile strength was around 1.0 in plasma, however, lincosamides treatment had a strong effect on the gut microbiome, as clearly shown by both the high number of total changes of metabolites (44-57\%) and the high values of the feces profile strength (3.5-4.0). This implies that only a fraction of the fecal metabolome changes has an influence on the plasma metabolome, given the fact that 
the same metabolites were measured in both samples. Since in this study the aim was to establish metabolome patterns of the plasma metabolites, changes of relevant metabolites in the lincosamides patterns were established.

\section{Impact of lincosamides on the gut microbiome and specific plasma metabolome patterns}

Specific plasma metabolome patterns were established in the MetaMap®Tox database to characterize the effects of the lincosamides antibiotics on the functionality of the gut microbiome. These patterns were created I) with the aim of identifying microbiome-related or -associated metabolites, and II) as a first step towards future identification of substances with similar effects on the microbiome in the MetaMap®Tox database, and. Different classes of antibiotics provide different patterns of microbiota alterations because of their different spectrum and antibacterial targets. In our previous study, it has been demonstrated that antibiotic class-specific changes in the plasma metabolome of rats can be detected (Behr et al., 2017).

In the present study, specific plasma metabolome patterns (for male and female animals) were established in the database for the influence of lincosamides antibiotics on the gut microbiome. These patterns are composed by potentially gut microbiome-related metabolites, which are believed to be either produced or further modified by the intestinal microbial communities.

The community analysis showed no significant differences between male and female control animals and female animals treated with CMC. Male animals treated with CMC showed a decrease of Rhodospirillales. However, due to the minor relative abundance of these Proteobacteria, the PCoA did not show a clear separation of CMC treated males from the control animals. After lincosamides treatment, the community analysis confirmed that at the compositional level the bacterial community was markedly affected and consequently the changes observed in the metabolome could be linked to a disruption on the gut microbiome. Lincosamides are particularly active against anaerobes, e.g. Bacteroidetes, which are the main colonizers of the gastrointestinal tract. This is consistent with the depletion of Bacteroidetes observed in this study. In line with our findings, in other studies levels of species belonging to Bacteroidetes significantly decreased whereas levels of Proteobacteria and Firmicutes markedly increased after exposure, and the total bacterial diversity was reduced compared to control animals (Hornish et al., 1987; Sun and Hsi, 1973). The diversity analysis presented here was carried out to demonstrate alterations in the gut microbiota that could be linked to the metabolic changes observed in the plasma and feces profiles. A deeper exploration into more specific taxonomic groups (e.g. genera or species) and their metabolic functionality was beyond the aims of the present study but will be performed in the future using data from studies with antibiotics having different activity spectra to allow a broad correlation analysis and an integration of these data into systems biology models. 


\section{Identification of key metabolites in MetaMap ${ }^{\circledR}$ Tox}

The plasma metabolome patterns for lincosamides showed changes of metabolites belonging mainly to the classes amino acids and related metabolites, bile acids and complex lipids, fatty acids and related metabolites. Some of the metabolites were found to be altered in both plasma and feces, which strengthens their linkage with the microbiome. Feces are composed by a large quantity of gut bacteria and their metabolic products. Lincosamides exerted a substantial effect on the feces metabolome of rats. The number of significantly upregulated metabolites, contrary to the observation in plasma, was much higher than the number of downregulated metabolites suggesting that either the lack of bacterial enzymes is causing an accumulation of these metabolites in the gut or the outgrowth of the communities not affected by the antibiotics is producing more of certain compounds. From the principal component analysis (PCA) clear treatment-related effects were revealed in the metabolite profile of feces after the antibiotics administration. An overall comparison of significant altered metabolites after lincosamides treatment where significant increased and decreased plasma metabolites were compared with the changes are shown in Table 4, where also feces metabolites that were found to be altered more than 5-fold were included. In the following sections amino acids and related as well as bile acids as key metabolites of the plasma patterns from different metabolite classes are discussed in detail.

\section{Amino acids and related metabolites}

Amino acids and proteins as an essential part of the diet reach the intestinal tract, and a considerable part reaches the colon encountering bacterial communities which make use of them for their metabolic processes. Studies in germ-free (GF) and conventionalized mice have demonstrated that the gut microbiota not just affects the bioavailability of host's amino acids but also alters the distribution of free amino acids in the GI tract (Macfarlane et al., 1988; Whitt and Demoss, 1975). After 28 days of lincosamides antibiotics exposure our metabolomics analysis revealed that several amino acids fluctuated after treatment. The plasma metabolic profiles showed that the amino acids glycine and tyrosine were present in lower concentrations compared to the controls while glutamate was found in higher concentrations. Conversely, in feces glycine, tyrosine, glutamate, taurine and tryptophan were significantly upregulated. On the one hand, lower levels in plasma accompanied by higher levels in feces suggest that these amino acids accumulated in the intestine and, with the exception of glutamate, were not absorbed into the system. Glycine, more likely as a consequence of bacterial impairment, could not be further metabolized and therefore accumulated in the intestine as indicated by its elevated levels in feces. On the other hand, due to less bacterial polysaccharide degradation, energy metabolism in the rat could have switched to amino acid and lipid metabolism. This could explain a decrease of amino acids and lipids, and an increase of glutamate in plasma which is produced by transferring excessive ammonia to alpha-ketoglutarat. Furthermore, critical for the whole-body metabolism, a decrease in glycine levels was observed in plasma. Given the importance and the wide range of metabolic pathways, e.g. the glutamatergic system, in which glycine is 
involved, a change in the concentration of this amino acid could have consequences for the host's well-being (Lechner, 2006). Creatine and creatinine, indole-derivates and hippuric acid were already identified as key metabolites and described in detail in our previous studies (Behr et al., 2017) (Behr et al, submitted).

\section{Bile acids}

After lincosamides treatment, primary bile acids were highly altered in both male and female animals in plasm and feces. In both matrices the taurine conjugated bile acid, taurocholic acid, was highly upregulated. The glycine conjugated glycochenodeoxycholic acid was downregulated in both plasma and feces. Plasma levels of glycochenodeoxycholic acid were particularly low, reaching undetectable levels. Cholic acid, however, presented an opposite pattern: it was upregulated in feces and downregulated in plasma. Similar perturbations of the bile acid pool after antibiotic treatment were already reported in different studies and associated with Clostridium difficile colonization (Cepa et al., 2018; Swann et al., 2011; Theriot et al., 2015). Increased levels of taurine conjugated bile acids and a decrease in glycine conjugated bile acids are generally observed as a consequence of gut microbiome impairments. In a metagenomic study it was shown that Firmicutes deconjugated both glycine and taurine bile acids, while Bacteroidetes were only capable of deconjugating taurine derivates (Jones et al., 2008). In rats, almost all the bile acids are conjugated with taurine rather than glycine, which implies that taurine conjugation dominates at physiological concentrations (Killenberg and Jordan, 1978). The community analysis after the administration of clindamycin and lincomycin revealed a depletion of Bacteroidetes and an increase of Firmicutes. This is consistent with the high levels of the conjugated taurocholic acid, due to the depletion of taurocholic acid-specific bile salt hydrolases (BSHs) exhibited by Bacteroidetes (Ridlon et al., 2006). The loss of Bacteroidetes-specific hydrolases that deconjugate taurocholic acid could account for the strikingly higher values of taurocholic acid observed in both plasma and feces. It is important to mention that taurocholic acid is transported actively from the intestine. Earlier studies have shown that taurocholic acid uptake is increased in the ileal epithelium of GF rats supporting the increased reabsorption which could be reflected in higher levels of this metabolite in plasma (Riottot and Sacquet, 1985). The increase of Firmicutes is in line with the decreased levels of glycochenodeoxycholic acid and the increased levels of glycine observed in feces.

Cholic acid accumulated in the intestine and it can be hypothesized that it could not be further converted into secondary bile acids due to the loss of microorganisms with a $7 \alpha-$ dehydroxylation activity. Moreover, cholic acid is a hydrophobic bile acid which is not actively transported. Therefore, cholic acid is in general inefficiently absorbed by passive diffusion, and moreover could be excreted in higher amounts due to higher levels of fatty acids like the TAGs. The reabsorption via the enterohepatic pathway could be less efficient which could explain the lower levels of this metabolite observed in the plasma of lincosamides treated animals. 
As already observed in our previous study, antibiotic treatment led to various significant alterations of lipids and fatty acids probably both due to the disruption of the bile acid metabolism and a decrease of microbial lipases or (Behr et al, submitted). Especially triacylglycerols (TAGs) were present at significantly higher levels in feces after the antibiotics administration. Another reason for these high levels in the intestine could be the TAG content in bacterial cells (Alvarez and Steinbüchel, 2003), which were destroyed by antibiotic treatment. In consequence these fatty acid triesters of glycerol could be released and could have led to an induction of bile acid production in the liver.

The changes observed in the primary bile acids after lincosamides administration suggest that the gut microbiome has a strong influence on the bile acid metabolism. Since bile acids are reabsorbed from the intestine, an altered bile acid metabolism and the gut microbiota have the potential to influence the gut-liver axis, the immune system and other body systems. Altered bile acid metabolism has been associated with diseases beyond the gastrointestinal tract, such as the non-alcoholic fatty liver disease (NAFLD), drug-induced liver injury or cardiovascular dysfunction (Cepa et al., 2018; Fu et al., 2016; Khurana et al., 2011). It is also conceivable that altered bile acid concentrations may affect the absorption of xenobiotics, with particular importance for pharmaceutical products. In the present study, the levels of secondary bile acids were not assessed. The measurement of these metabolites could be essential for achieving a comprehensive understanding of the interplay between bile acids and the gut microbiome.

\section{CONCLUSION}

In this study, specific plasma patterns for the lincosamides effects on the gut microbiome were established, potentially microbiome-derived or -associated metabolites in both plasma and feces could be identified and a profound impact of lincosamides on the bacterial communities was demonstrated. The diversity of the community was drastically narrowed down, and major shifts were observed in bacterial phyla. For example, Firmicutes represented greater than $80 \%$ of the total diversity in antibiotic-treated animals compared to around $40 \%$ in control animals. The vehicle CMC showed negligible effects on both the metabolome and microbial communities. Most changes could be observed in metabolites belonging to the class of bile acids, complex lipids, fatty acids and related metabolites, as well as amino acids and related metabolites. The principal component analysis showed a clear treatment-related effect on the feces metabolome, and most of the altered metabolites were increased. Taken together, it is demonstrated that the gut microbiota influences the host metabolic phenotype by producing or modifying some metabolites that can be taken up into the system and consequently be detected in plasma. The creation of metabolic patterns associated to different types of microbiome disruption represents the first step towards assessing the toxicological profiles of compounds or biologicals regarding the gut microbial communities. 
Acknowledgement: We would like to thank Dr. Saskia Sperber, Dr. Hennicke Kamp and Dr. Burkhard Flick for proofreading the manuscript and valuable discussions, and Ms Irmgard Weber for her skillful assistance in taking plasma samples.

Funding information: The authors have no relevant affiliations or financial involvement with any organization or entity with a financial interest in or financial conflict with the subject matter or materials discussed in the manuscript. This includes employment, consultancies, honoraria, stock ownership or options, expert testimony, grants or patents received or pending, or royalties. No writing assistance was utilized in the production of this manuscript.

\section{Appendix A. Supplementary data}

Supplementary data to this article can be found online at doi:https://doi.org/10.1016/j.toxlet.2018.08.002. 


\section{REFERENCES}

Alvarez, H.M., Steinbüchel, A., 2003. Triacylglycerols in prokaryotic microorganisms. Appl. Microbiol. Biotechnol. 60, 367-376. https://doi.org/10.1007/s00253-002-11350

Anderson, M.J., 2017. Permutational Multivariate Analysis of Variance (PERMANOVA). Wiley StatsRef Stat. Ref. Online https://doi.org/10.1002/9781118445112.stat07841

Bäumler, A.J., Sperandio, V., 2016. Interactions between the microbiota and pathogenic bacteria in the gut. Nature 535, 85-93. https://doi.org/10.1038/nature18849

Behr, C., Kamp, H., Fabian, E., Krennrich, G., Mellert, W., Peter, E., Strauss, V., Walk, T., Rietjens, I.M.C.M., van Ravenzwaay, B., 2017. Gut microbiome-related metabolic changes in plasma of antibiotic-treated rats. Arch. Toxicol. 91, 3439-3454. https://doi.org/10.1007/s00204-017-1949-2

Callahan, B.J., McMurdie, P.J., Rosen, M.J., Han, A.W., Johnson, A.J.A., Holmes, S.P., 2016. DADA2: High resolution sample inference from Illumina amplicon data. Nat Methods. 13, 581-583. https://doi.org/10.1016/S2215-0366(16)30284X.Epidemiology

Caporaso, J.G., Kuczynski, J., Stombaugh, J., Bittinger, K., Bushman, F.D., Costello, E.K., Fierer, N., Pẽa, A.G., Goodrich, J.K., Gordon, J.I., Huttley, G.A., Kelley, S.T., Knights, D., Koenig, J.E., Ley, R.E., Lozupone, C.A., McDonald, D., Muegge, B.D., Pirrung, M., Reeder, J., Sevinsky, J.R., Turnbaugh, P.J., Walters, W.A., Widmann, J., Yatsunenko, T., Zaneveld, J., Knight, R., 2010. QIIME allows analysis of highthroughput community sequencing data. Nat. Methods. https://doi.org/10.1038/nmeth.f.303

Cepa, S., Potter, D., Wong, L., Schutt, L., Tarrant, J., Pang, J., Zhang, X., Andaya, R., Salphati, L., Ran, Y., An, L., Morgan, R., Maher, J., 2018. Individual serum bile acid profiling in rats aids in human risk assessment of drug-induced liver injury due to BSEP inhibition. Toxicol. Appl. Pharmacol. 338, 204-213. https://doi.org/10.1016/J.TAAP.2017.11.007

Chao, A., Chiu, C.-H., Jost, L., 2010. Phylogenetic diversity measures based on Hill numbers. Philos. Trans. R. Soc. B Biol. Sci. 365, 3599-3609. https://doi.org/10.1098/rstb.2010.0272

Chassaing, B., Koren, O., Goodrich, J.K., Poole, A.C., Srinivasan, S., Ley, R.E., Gewirtz, A.T., 2015. Dietary emulsifiers impact the mouse gut microbiota promoting colitis and metabolic syndrome. Nature 519, 92-96. https://doi.org/10.1038/nature14232

Cho, I., Blaser, M.J., 2012. The human microbiome: at the interface of health and disease. Nat. Rev. Genet. 13, 260-70. https://doi.org/10.1038/nrg3182

Edgar, R., 2016. SINTAX: a simple non-Bayesian taxonomy classifier for 16S and ITS sequences. bioRxiv 74161. https://doi.org/10.1101/074161

Fu, T., Zhao, X., Evans, R.M., 2016. Liver Cancer Checks in When Bile Acid Clocks Out. Cancer Cell 30, 827-828. https://doi.org/10.1016/j.ccell.2016.11.012

Gilbert, J.A., Quinn, R.A., Debelius, J., Xu, Z.Z., Morton, J., Garg, N., Jansson, J.K., Dorrestein, P.C., Knight, R., 2016. Microbiome-wide association studies link 
dynamic microbial consortia to disease. Nature. https://doi.org/10.1038/nature18850

Gray, J.E., Purmalis, A., Feenstra, E.S., 1964. Animal Toxicity Studies of a New Antibiotic, Lincomycin. Toxicol Appl Pharmacol 6, 476-496.

Gray, J.E., Purmalis, A., Mulvihill, W.J., 1966. Further toxicologic studies of lincomycin. Toxicol. Appl. Pharmacol. 9, 445-454. https://doi.org/10.1016/0041-008X(66)900032

Gray, J.E., Purmalis, A., Purmalis, B., Mathews, J., 1971. Ultrastructural studies of the hepatic changes brought about by clindamycin and erythromycin in animals. Toxicol. Appl. Pharmacol. 19, 217-233. https://doi.org/10.1016/0041-008X(71)90108-6

Gray, J.E., Weaver, J.A., Feenstra, E.S., 1972. The Oral Toxicity of Clindamycin in Laboratory Animals. Toxicol Appl Pharmacol 21, 516-531.

Hornish, R.E., Ron, S., Nappier, J.M., Gosline, E., 1987. Comparative metabolism of lincomycin in the swine chicken, and cat. Drug Metab. Rev. 18, 177-214. https://doi.org/10.3109/03602538708998305

Hylemon, P.B., Zhou, H., Pandak, W.M., Ren, S., Gil, G., Dent, P., 2009. Bile acids as regulatory molecules. J. Lipid Res. 50, 1509-1520. https://doi.org/10.1194/jlr.R900007-JLR200

Jones, B. V., Begley, M., Hill, C., Gahan, C.G.M., Marchesi, J.R., 2008. Functional and comparative metagenomic analysis of bile salt hydrolase activity in the human gut microbiome. Proc. Natl. Acad. Sci. U. S. A. 105, 13580-5. https://doi.org/10.1073/pnas.0804437105

Kamp, H., Strauss, V., Wiemer, J., Leibold, E., Walk, T., Mellert, W., Looser, R., Prokoudine, A., Fabian, E., Krennrich, G., Herold, M., Ravenzwaay, B. van, 2012. Reproducibility and robustness of metabolome analysis in rat plasma of 28-day repeated dose toxicity studies. Toxicol. Lett. 215, 143-149.

Khurana, S., Raufman, J.P., Pallone, T.L., 2011. Bile acids regulate cardiovascular function. Clin. Transl. Sci. 4, 210-218. https://doi.org/10.1111/j.17528062.2011.00272.x

Killenberg, P.G., Jordan, J.T., 1978. Purification and Characterization of Rile Acid-CoA : Acid N-Acyltransferase from Rat Liver *. J. Biol. Chem. 253, 1005-1010.

Lechner, S.M., 2006. Glutamate-based therapeutic approaches: Inhibitors of glycine transport. Curr. Opin. Pharmacol. https://doi.org/10.1016/j.coph.2005.11.002

Macfarlane, G.T., Allison, C., Gibson, S.A.W., Cummings, J.H., 1988. Contribution of the microflora to proteolysis in the human large intestine. J. Appl. Bacteriol. 64, 37-46. https://doi.org/10.1111/j.1365-2672.1988.tb02427.x

Makishima, M., Lu, T.T., Xie, W., Whitfield, G.K., Domoto, H., Evans, R.M., Haussler, M.R., Mangelsdorf, D.J., 2002. Vitamin D receptor as an intestinal bile acid sensor. Science (80-. ). 296, 1313-1316. https://doi.org/10.1126/science.1070477

Mandal, S., Van Treuren, W., White, R.A., Eggesbø, M., Knight, R., Peddada, S.D., 2015. Analysis of composition of microbiomes: a novel method for studying microbial composition. Microb. Ecol. Heal. Dis. 26, 1-7. https://doi.org/10.3402/mehd.v26.27663 
Martin, M., 2011. Cutadapt removes adapter sequences from high-throughput sequencing reads. EMBnet.journal 17, 10. https://doi.org/10.14806/ej.17.1.200

Martino, J.V., Van Limbergen, J., Cahill, L.E., 2017. The Role of Carrageenan and Carboxymethylcellulose in the Development of Intestinal Inflammation. Front. Pediatr. 5, 1-7. https://doi.org/10.3389/fped.2017.00096

Maruyama, T., Miyamoto, Y., Nakamura, T., Tamai, Y., Okada, H., Sugiyama, E., Nakamura, T., Itadani, H., Tanaka, K., 2002. Identification of membrane-type receptor for bile acids (M-BAR). Biochem. Biophys. Res. Commun. 298, 714-719. https://doi.org/10.1016/S0006-291X(02)02550-0

Mattes, W., Davis, K., Fabian, E., Greenhaw, J., Herold, M., Looser, R., Mellert, W., Groeters, S., Marxfeld, H., Moeller, N., Montoya-Parra, G., Prokoudine, a., van Ravenzwaay, B., Strauss, V., Walk, T., Kamp, H., 2014. Detection of hepatotoxicity potential with metabolite profiling (metabolomics) of rat plasma. Toxicol. Lett. 230, 467-478. https://doi.org/10.1016/j.toxlet.2014.07.021

McCoy, K.D., Geuking, M.B., Ronchi, F., 2017. Gut microbiome standardization in control and experimental mice. Curr. Protoc. Immunol. 2017, 23.1.1-23.1.13. https://doi.org/10.1002/cpim.25

QIIME2 [WWW Document], n.d. URL https://qiime2.org/ (accessed 2.6.18).

Ridlon, J.M., Kang, D.-J., Hylemon, P.B., 2006. Bile salt biotransformations by human intestinal bacteria. J. Lipid Res. 47, 241-259. https://doi.org/10.1194/jlr.R500013JLR200

Riottot, M., Sacquet, E., 1985. Increase in the ileal absorption rate of sodium taurocholate in germ-free or conventional rats given an amylomaize-starch diet. Br. J. Nutr. 53, 307-310. https://doi.org/10.1079/BJN19850038

Roessner, U., Wagner, C., Kopka, J., Trethewey, R.N., Willmitzer, L., 2000. Technical advance: simultaneous analysis of metabolites in potato tuber by gas chromatography-mass spectrometry. Plant J. 23, 131-42. https://doi.org/10.1046/j.1365-313x.2000.00774.x

Stacklies, W., Redestig, H., Scholz, M., Walther, D., Selbig, J., 2007. pcaMethods - A bioconductor package providing PCA methods for incomplete data. Bioinformatics 23, 1164-1167. https://doi.org/10.1093/bioinformatics/btm069

Strauss, V., Mellert, W., Wiemer, J., Leibold, E., Kamp, H., Walk, T., Looser, R., Prokoudine, a., Fabian, E., Krennrich, G., Herold, M., Van Ravenzwaay, B., 2012. Increased toxicity when fibrates and statins are administered in combination - A metabolomics approach with rats. Toxicol. Lett. 211, 187-200. https://doi.org/10.1016/j.toxlet.2012.03.798

Sun, F.F., Hsi, R.S.P., 1973. Metabolism of clindamycin I: Absorption and excretion of clindamycin in rat and dog. J. Pharm. Sci. 62, 1265-1269. https://doi.org/10.1002/jps.2600620806

Swann, J.R., Want, E.J., Geier, F.M., Spagou, K., Wilson, I.D., Sidaway, J.E., Nicholson, J.K., Holmes, E., 2011. Systemic gut microbial modulation of bile acid metabolism in host tissue compartments. Proc. Natl. Acad. Sci. 108, 4523-4530. https://doi.org/10.1073/pnas.1006734107 
Theriot, C.M., Bowman, A. a, Young, V.B., 2015. Antibiotic-Induced Alterations of the Gut Microbiota Alter Secondary Bile Acid Production and Allow for Clostridium difficile Spore Germination and Outgrowth in the Large Intestine. mSphere 1, e00045-15. https://doi.org/10.1128/mSphere.00045-15.Editor

Troyanskaya, O., Cantor, M., Sherlock, G., Brown, P., Hastie, T., Tibshirani, R., Botstein, D., Altman, R.B., 2001. Missing value estimation methods for DNA microarrays. Bioinformatics 17, 520-525. https://doi.org/10.1093/bioinformatics/17.6.520

van Ravenzwaay, B., Coelho-Palermo Cunha, G., Strauss, V., Wiemer, J., Leibold, E., Kamp, H., Walk, T., Mellert, W., Looser, R., Prokoudine, a., Fabian, E., Krennrich, G., Herold, M., 2010. The individual and combined metabolite profiles (metabolomics) of dibutylphthalate and di(2-ethylhexyl)phthalate following a 28-day dietary exposure in rats. Toxicol. Lett. 198, 159-170. https://doi.org/10.1016/j.toxlet.2010.06.009

van Ravenzwaay, B., Cunha, G.C.P., Leibold, E., Looser, R., Mellert, W., Prokoudine, a., Walk, T., Wiemer, J., 2007. The use of metabolomics for the discovery of new biomarkers of effect. Toxicol. Lett. 172, 21-28. https://doi.org/10.1016/j.toxlet.2007.05.021

van Ravenzwaay, B., Herold, M., Kamp, H., Kapp, M.D., Fabian, E., Looser, R., Krennrich, G., Mellert, W., Prokoudine, A., Strauss, V., Walk, T., Wiemer, J., 2012. Metabolomics: A tool for early detection of toxicological effects and an opportunity for biology based grouping of chemicals-From QSAR to QBAR. Mutat. Res. - Genet. Toxicol. Environ. Mutagen. 746, 144-150. https://doi.org/10.1016/j.mrgentox.2012.01.006

van Ravenzwaay, B., Kamp, H., Montoya-parra, G.A., Strauss, V., Fabian, E., Mellert, W., Walk, T., Peter, E., Looser, R., Herold, M., 2016. The development of a database for metabolomics - looking back on ten years of experience. Int. J. Biotechnol. 14, 4768. https://doi.org/10.1504/IJBT.2015.074801

Vítek, L., Haluzík, M., 2016. The role of bile acids in metabolic regulation. J. Endocrinol. 228, R85-R96. https://doi.org/10.1530/JOE-15-0469

Walk, T.B., Dostler, M., 2003. Mass spectrometry method for analysing mix- tures of substances, Patent application PCT/EP2003/001274. WO2003073464, Metanomics, Berlin.

Walk, T.B., Looser, R., Bethan, B., Herold, M.M., Kamlage, B., Schmitz, O., van Ravenzwaay, B., Mellert, W., Coelho, P.C.G., Ehrhardt, T., Wiemer, J., Prokoudine, A., Krennrich, G., 2006. Means and methods for analyzing a sample by means of chromatography-mass spectrometry, Patent application PCT/EP2006/064628. WO2007012643A1, Metanomics, Berlin.

Whitt, D.D., Demoss, R.D., 1975. Effect of microflora on the free amino acid distribution in various regions of the mouse gastrointestinal tract. Appl. Microbiol. 30, 609-15.

Wikoff, W.R., Anfora, A.T., Liu, J., Schultz, P.G., Lesley, S. a, Peters, E.C., Siuzdak, G., 2009. Metabolomics analysis reveals large effects of gut microflora on mammalian blood metabolites. Proc. Natl. Acad. Sci. U. S. A. 106, 3698-3703. https://doi.org/10.1073/pnas.0812874106

Xie, W., Radominska-Pandya, A., Shi, Y., Simon, C.M., Nelson, M.C., Ong, E.S., 
Waxman, D.J., Evans, R.M., 2001. An essential role for nuclear receptors SXR/PXR in detoxification of cholestatic bile acids. Proc. Natl. Acad. Sci. 98, 3375-3380. https://doi.org/10.1073/pnas.051014398

Yilmaz, P., Parfrey, L.W., Yarza, P., Gerken, J., Pruesse, E., Quast, C., Schweer, T., Peplies, J., Ludwig, W., Glöckner, F.O., 2014. The SILVA and "all-species Living Tree Project (LTP)" taxonomic frameworks. Nucleic Acids Res. 42. https://doi.org/10.1093/nar/gkt1209 



\section{Chapter 5}

Analysis of metabolome changes in the bile acid pool in feces and plasma of antibiotic-treated rats

C. Behr, M. Slopianka, V. Haake, V. Strauss, S. Sperber, H. Kamp, T. Walk, K. Beekmann, I.M.C.M. Rietjens, B. van Ravenzwaay

Published in:

Toxicology and Applied Pharmacology 363 (2019) 79-87 


\section{Abstract}

The bile acid-liver-gut microbiota axis plays an important role in the host's health. The gut microbiota has an impact on the bile acid pool, but also the bile acids themselves can influence the gut microbiota composition. In this study, six antibiotics from five different classes (i.e. lincosamides, glycopeptides, macrolides, fluoroquinolones, aminoglycosides) were used to modulate microbial communities of Wistar rats to elucidate changes in the bile acid metabolism and to identify key metabolites in the bile acid pool related to gut microbial changes. 20 primary and secondary bile acids were analyzed in plasma and feces of control and treated animals. Antibiotics treatment induced significant changes in primary and secondary bile acids in both matrices. Taurine-conjugated primary bile acids significantly increased in plasma and feces. Contrary, cholic acid and most of the analyzed secondary bile acids significantly decreased in plasma, and cholic acid accumulated in the feces after treatment with all antibiotics but roxithromycin. Despite the different activity spectra of the antibiotics applied against gut microbes, the overall effect on the bile acid pool tended to be similar in both matrices except for streptomycin. These results show that changes in the gut microbial community affect the bile acid pool in plasma and feces and that changes in the bile acid profile can be indicative of alterations of the gut microbiome. Due to the important role of bile acids for the host, changes in the bile acid pool can have severe consequences for the host. 
Key words: metabolomics; gut microbiome; bile acid profiling; repeated dose oral toxicity study; antibiotics; microbiome-related metabolites

$\begin{array}{ll}\text { ABBREVIATIONS } \\ \text { BSH } & \text { bile salt hydrolase } \\ \text { GC } & \text { gas chromatography } \\ \text { GF } & \text { germ-free } \\ \text { HCA } & \text { hierarchical clustering analysis } \\ \text { IBD } & \text { inflammatory bowel disease } \\ \text { IBS } & \text { irritable bowel syndrome } \\ \text { LC } & \text { liquid chromatography } \\ \text { MS } & \text { mass spectrometry } \\ \text { OECD } & \text { Organisation for Economic Co-operation and Development } \\ \text { PCA } & \text { principal component analysis } \\ \text { SBS } & \text { short bowel syndrome } \\ \text { SPE } & \text { solid phase extraction }\end{array}$

\section{INTRODUCTION}

The gut microbiome has a strong influence on mammalian blood metabolites, especially on components of the bile (Long et al., 2017). Bile acids, which are the major functional components of the bile, are important signaling molecules and distinctive representors of the tight interplay between microbes and the host. Via complex molecular mechanisms they can influence the gut-liver axis, the immune system and other systems of the body. It is also conceivable that altered bile acid concentrations may affect the absorption of other metabolites or xenobiotics which ultimately may lead to altered plasma concentrations.

Primary bile acids are synthesized in the liver from cholesterol and are conjugated with either taurine or glycine (such as chenodeoxycholate (CDCA), tauro- and glycochenodeoxycholate (TCDCA, GCDCA), cholate (CA), tauro- and glycocholate (TCA, GCA), $\alpha$ - and $\beta$-muricholate and its taurine conjugate ( $\alpha$-, $\beta$-MCA and -TMCA)), thereby gaining their amphiphilic character, before they are secreted into the bile and finally into the small intestine to emulsify lipids into micelles and assist in nutrient absorption. Lipids are emulsified by bile salts for the optimal hydrolysis activity of endogenous or microbial lipases, e.g converting triacylglycerols to monoacyl- and diacylglycerols, fatty acids and glycerol. The majority of bile acids is actively reabsorbed in the ileum. A minor amount escapes reabsorption, reaches the colon and is partly excreted. When reaching the intestine, primary bile acids are further metabolized by unique microbial enzymes to unconjugated, secondary and tertiary bile acids (such as deoxycholate (DCA), tauro- and glycodeoxycholate (GDCA, TDCA), $\omega$-muricholate ( $\omega$-MCA), hyodeoxycholate (HDCA), ursodeoxycholate (UDCA), tauro- and glycoursodeoxycholic acid (TUDCA, GUDCA), lithocholate (LCA), tauro- and glycolithocholic aicd (TLCA, GLCA)). The microorganisms 
possess enzymes to modify these bile acids, for example bile salt hydrolase (BSH) that cleaves the peptide linkage between the amino acid and the primary bile acid. Furthermore, some microbes, mainly anaerobes, possess a $7 \alpha$-dehydroxylation activity to convert primary bile acids into secondary bile acids (Ridlon et al., 2006). The bile acids can enter the enterohepatic circulation via active or passive transport, are recycled and re-conjugated in the liver, and then re-secreted into the bile and the intestine (see Figure 1).

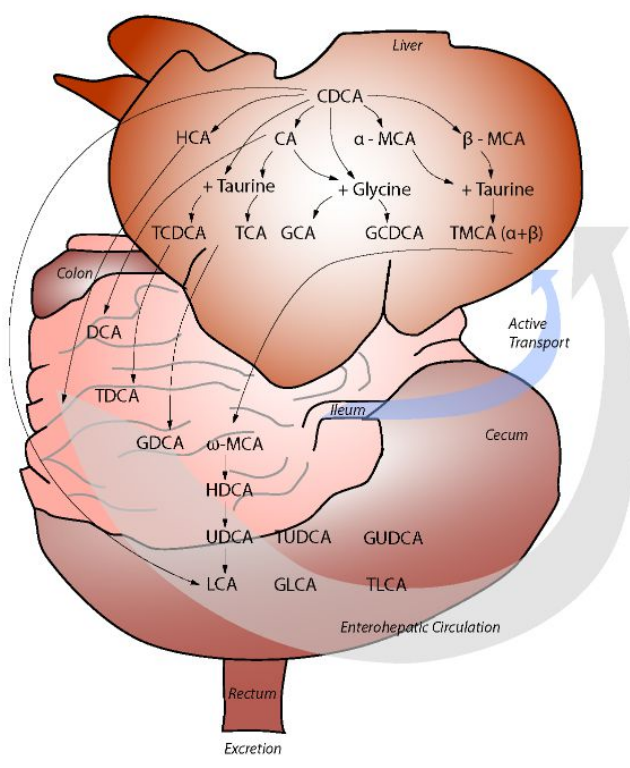

Figure 1 Scheme of primary, secondary and tertiary bile acids that were measured in rat feces and plasma in this study. Primary bile acids are produced in the liver and conjugated with taurine or glycine, then excreted into the intestine and modified by microbes. The major part is actively reabsorbed in the intestine and the remaining bile acids are passively reabsorbed, entering the enterohepatic pathway.
Although bile acids have a key role in lipid digestion and absorption, they are also recognized as important signaling molecules serving as ligands for numerous nuclear receptors, e.g. the farnesoid $\mathrm{X}$ receptor (FXR), the trans-membrane-bound Gprotein-coupled receptor (TGR5), the vitamin D receptor (VDR) and pregnane $\mathrm{X}$ receptor (PXR) (Makishima et al., 2002; Maruyama et al., 2002; Xie et al., 2001). Through binding to these receptors, bile acids can regulate genes involved in lipid and glucose metabolism, and energy homeostasis (Hylemon et al., 2009).

Regarding the mechanisms of bile acids binding to cytoplasmic and nuclear receptors they have been proposed to act in a way similar to that of hormones (Vítek and Haluzík, 2016). Perturbations of the bile acid pool are associated to several gastrointestinal diseases such as Clostridium difficile infection, irritable bowel syndrome (IBS), inflammatory bowel diseases (IBD), short bowel syndrome (SBS) and even colorectal cancer (Dior et al., 2016; Duboc et al., 2013; Pereira-Fantini et al., 2016; Theriot et al., 2015; Zou et al., 2017). Furthermore, via the enterohepatic circulation, an altered bile acid metabolism has an effect on the gut-liver axis and other body systems, and has been associated with several diseases beyond the gastrointestinal tract such as non-alcoholic fatty liver disease (NAFLD), druginduced liver injury (DILI), or cardiovascular dysfunction (Khurana et al., 2011; Mouzaki et al., 2016; Slopianka et al., 2017). 
In a previous study, we performed metabolite profiling in plasma and feces of rats after lincosamides treatment in order to establish specific metabolite patterns in the MetaMap ${ }^{\circledR}$ Tox database and to identify key metabolites indicating gut microbial changes (Behr et al., 2018). The results showed that after treatment, primary bile acids were highly altered in both male and female animals in plasma and feces. However, the levels of other primary bile acids and secondary bile acids, which are known to be produced exclusively by the microbes in the gut, were not assessed. Therefore, in this study the aim was to further elucidate changes in the bile acid metabolism and to identify key metabolites related to gut microbial changes. Primary and secondary bile acids in plasma and feces of rats were analyzed after treatment with different classes of antibiotics. The evaluation of changes in the plasma and feces bile acid pool could help to gain a more comprehensive understanding of how microbial changes may influence the bile acid metabolism which, regarding the important role of these signaling molecules, can have far reaching consequences for the host.

\section{Material ANd Methods}

Three independent studies were performed according to the Organisation for Economic Cooperation and Development (OECD) 407 protocol. Animal handling, treatment, and clinical examinations have been described earlier (Kamp et al., 2012; Strauss et al., 2012; van Ravenzwaay et al., 2012, 2010, 2007).

\section{Ethics statement}

The studies were approved by the BASF Animal Welfare Body, with the permission of the local authority, the Landesuntersuchungsamt Rheinland-Pfalz (approval number 23177 07/G 13-3-016). The study was performed in an AAALAC-approved (Association for Assessment and Accreditation of Laboratory Animal Care International) laboratory in accordance with the German Animal Welfare Act and the effective European Council Directive.

\section{Animals and maintenance conditions}

Briefly, male and female Wistar rats (CrI:WI(Han)) were supplied by Charles River, Germany, and were $70 \pm 1$ days old at the beginning of the studies. The animals ( 5 rats per sex and cage in one group) were maintained in an air-conditioned room at a temperature of 20 to $24^{\circ} \mathrm{C}$, a relative humidity of 30 to $70 \%$, and a 12 hour light / 12 hour dark cycle. Ground Kliba mouse/rat maintenance diet "GLP" was supplied by Provimi Kliba SA, Kaiseraugst, Switzerland. Diet and drinking water were available ad libitum (except 16 - 20 hours before sampling) and regularly assayed for chemical contaminants and the presence of microorganisms. 


\section{Treatment of animals with compounds}

The antibiotics were administered daily by gavage using an appropriate vehicle to each treatment group consisting of five rats per group and sex, and metabolite and bile acid changes were compared with a dietary control group (10 rats per sex) (see Table 1). Dose levels of the antibiotics were selected in such a way that the dose would induce a clear effect on the targeted microbiome population, without causing systemic toxicity (Gray et al., 1972, 1971, 1966, 1964; Ho et al., 2015; Villa et al., 1988; von Keutz and Schlüter, 1999; World Health Organization, 1997). To examine that the effect of the vehicle carboxymethylcellulose (CMC), which was used for the administration of some of the administered antibiotics with limited water solubility, does not interfere with metabolite changes in the plasma patterns arisen from antibiotic treatment, 10 animals per sex received $10 \mathrm{ml} / \mathrm{kg}$ bw/d drinking water containing $0.5 \% \mathrm{CMC}$. The standard procedure for the evaluation of test substance-related effects in the MetaMap®Tox database is the comparison of the resulting values of the treatment groups against the baseline values of a diet control. This procedure has been used since 2004 resulting in a very extensive database of control values for the metabolites evaluated. As described in van Ravenzwaay et al. (van Ravenzwaay et al., 2016), this allows to determine not only the statistical significance of regulated metabolites, but also the assessment if a particular metabolite value has ever been observed in control animals - providing a historical range of what is normal. The substances were each administered in three separate and consecutive studies (study 1: vancomycin, streptomycin, roxithromycin, $0.5 \% \mathrm{CMC}$; study 2: sparfloxacin, lincomycin; study 3: clindamycin), each with a diet control group of 10 animals per sex, to allow for such comparisons. Dose-levels, routes of administration and form of preparation of the antibiotics are summarized in Table 1.

Table 1 Compounds used, dose levels, form of preparation, and class of antibiotics. All compounds were administered orally by gavage.

\begin{tabular}{|c|c|c|c|}
\hline Treatment & Dose/day & Form of preparation & Class of antibiotics \\
\hline Clindamycin & $200 \mathrm{mg} / \mathrm{kg}$ body weight & in ultra-pure water & Lincosamides \\
\hline Lincomycin & $300 \mathrm{mg} / \mathrm{kg}$ body weight & $\begin{array}{l}\text { in water containing } \\
0.5 \% \mathrm{CMC}^{\mathrm{a}}\end{array}$ & Lincosamides \\
\hline Roxithromycin & $200 \mathrm{mg} / \mathrm{kg}$ body weight & $\begin{array}{l}\text { in water containing } \\
0.5 \% \mathrm{CMC}^{\mathrm{a}}\end{array}$ & Macrolides \\
\hline Sparfloxacin & $200 \mathrm{mg} / \mathrm{kg}$ body weight & $\begin{array}{l}\text { in water containing } \\
0.5 \% \mathrm{CMC}^{\mathrm{a}}\end{array}$ & Fluoroquinolones \\
\hline Streptomycin & $100 \mathrm{mg} / \mathrm{kg}$ body weight & $\begin{array}{l}\text { in water containing } \\
0.5 \% \mathrm{CMC}^{\mathrm{a}}\end{array}$ & Aminoglycosides \\
\hline Vancomycin & $50 \mathrm{mg} / \mathrm{kg}$ body weight & in ultra-pure water & Glycopeptides \\
\hline
\end{tabular}

${ }^{a}$ carboxymethyl cellulose: Tylose CB30000 


\section{Sampling of blood and feces for bile acid profiling}

Between 7:30 and 10:30 h, on study days 7, 14 and 28 blood samples were taken from the retro-orbital sinus in all rats under isoflurane anesthesia (1.0 ml K-EDTA blood). The blood samples were centrifuged $\left(10^{\circ} \mathrm{C}, 20,000 \mathrm{x} \mathrm{g}, 2 \mathrm{~min}\right)$ and the EDTA plasma was separated. The EDTA plasma samples were covered with nitrogen and frozen at $-80{ }^{\circ} \mathrm{C}$ until bile acid profiling was performed.

Feces samples were carefully removed during necropsy on day 28 from the rectum after a fasting period of $16-20$ hours and one day after the last administration of the test substances. The samples were collected in pre-cooled (dry-ice) vials, immediately snapfrozen in liquid nitrogen and stored at $-80{ }^{\circ} \mathrm{C}$ until bile acid profiling was performed.

\section{Clinical examinations}

All animals were checked daily for any clinically abnormal signs and mortalities. Food consumption was determined on study days 6, 13 and 27. Body weight was determined before the start of the administration period in order to randomize the animals and on study days 6,13 and 27. At the end of the treatment period, the animals were sacrificed by decapitation under isoflurane anesthesia. Bile acid profiling of plasma and feces samples were performed for all control and treated animals.

\section{Bile acid profiling in plasma and feces}

Bile acid analysis was performed with a UHPLC-ESI-MS/MS consisting of a Waters Acquity UHPLC system coupled with an SCIEX 5500 Triple Quad ${ }^{\mathrm{TM}}$ LC-MS/MS system equipped with an ESI ion source which enabled the measurement of a total number of 20 bile acids. To ensure accuracy and precision, the method provides seven calibration standards, a mixture of 3 isotope-labeled internal standards, and a quality control sample. Prior to analysis $5 \mathrm{mg}$ of dried feces were extracted with $1 \mathrm{~mL}$ extraction solvent (ethanol $[95 \%] / \mathrm{NaOH}[0.1 \mathrm{~N}])$ in an ultrasonic bath for 30 minutes following a 10 minute centrifugation $\left(14,000 \mathrm{rpm}, 4^{\circ} \mathrm{C}\right)$. The supernatant was removed and used for analysis. 10 $\mu \mathrm{l}$ of plasma or extracted feces was added together with $10 \mu \mathrm{L}$ of internal standards mixture onto filter spots suspended in the wells of a 96-well filter plate (PALL AcroPrep, PTFE 0.2 $\mu \mathrm{m})$ fixed on top of a deep-well plate and extracted with $100 \mu \mathrm{L}$ methanol by shaking at $600 \mathrm{rpm}$ for $20 \mathrm{~min}$ on an Eppendorf ThermoMixer C (Eppendorf AG, Hamburg). Elution of the methanol extracts was performed by centrifugation $(5,700 \mathrm{rpm}, 5 \mathrm{~min})$ into the lower receiving deep-well plate, which was then detached from the upper filter plate. After adding $60 \mu \mathrm{L}$ Milli- $\mathrm{Q}^{\circledR}$ water to the extracts and shaking briefly (600 rpm, $5 \mathrm{~min}$ ), the sample plate was analyzed by LC-MS/MS. All target isobaric bile acids were baseline separated under ultrahigh pressure liquid chromatography (UPLC) conditions as described previously (Slopianka et al., 2017). Briefly, UPLC systems were used at a flow rate of $0.5-1 \mathrm{~mL} / \mathrm{min}$. Mobile phase A was water with $0.01 \%$ formic acid and $10 \mathrm{mM}$ ammonium acetate and mobile phase B was 30\% (v/v) acetonitrile / methanol with $0.01 \%$ formic acid and $10 \mathrm{mM}$ ammonium acetate. The gradient program initially started at $35 \% \mathrm{~B}$, increased to $100 \% \mathrm{~B}$ 
in $3.5 \mathrm{~min}$ held at $100 \% \mathrm{~B}$ for $0.5 \mathrm{~min}$, decreased to $35 \% \mathrm{~B}$ in $0.1 \mathrm{~min}$, and then held at $35 \% \mathrm{~B}$ for $0.9 \mathrm{~min}$, enabling a short runtime of $5 \mathrm{~min}$. Chromatographic separation was performed with a proprietary reversed- phased UHPLC analytical column (Biocrates Life Sciences AG, Innsbruck) kept at $50{ }^{\circ} \mathrm{C}$. Chromatographic performance was enhanced with a SecurityGuard ULTRA Cartridge C28/ XB-C18 for $2.1 \mathrm{~mm}$ ID precolumn (Phenomenex Cat. No. AJ0-8782). An injection volume of $5 \mu \mathrm{L}$ was used. Mass spectrometric detection was accomplished with electrospray ionization in negative ion mode. Two MRM transitions are used for each target bile acid for semi-quantitative evaluation.

\section{Statistics}

The data were analyzed by univariate and multivariate statistical methods. The sex- and day-stratified heteroscedastic t-test ("Welch test") was applied to compare metabolite levels of dose groups with respective controls. For all metabolites, changes were calculated as the ratio of the median of metabolite levels in individual rats in a treatment group relative to median of metabolite levels in rats in a matched control group (time point and sex). Hereafter, these ratios are referred to as 'relative abundance' in plasma or feces.

Principal Component Analysis (PCA) was done in SIMCA (Sartorius Stedim Biotech, Umetrics $^{\circledR}$ Suite of Data Analytics Solutions, version 15) using the default software settings (UV-scaling, centering, removal of samples and metabolites with more than $50 \%$ missing values). For the PCA, $\log 10$-transformed, sex- and day-stratified data normalized to the median of the corresponding control were used as input.

Hierarchical clustering analysis (HCA) was performed in TIBCO $^{\circledR}$ Spotfire ${ }^{\circledR}$ (TIBCO Spotfire Inc., version 6.0) using t-values from the Welch test (see above for details) as input. Row (samples) and column (metabolites) clustering was done using Ward's method for clustering with half square Euclidean distance measure based on Z-score normalized data where missing values were imputed as row average. Ratios are shown $\log 10$ transformed.

The term "significantly" is used to denote "statistically significantly" at the displayed $p$ level.

\section{Figures}

Figure 1 showing the enterohepatic circulation in rats was created using Adobe Illustrator CS5. 


\section{RESUltS}

\section{Clinical signs}

There were no mortalities in any of the treatment groups, except for one animal at the beginning of the study in the female group of streptomycin (not treatment-related). Animals treated with roxithromycin showed slight salivation immediately after administration. The group of female animals treated with clindamycin showed temporary clinical signs as indicated by salivation and semi closed eyelid ( 4 animals), and fur piloerection (1 animal). Similarly, males treated with clindamycin showed slight salivation (all animals), semi closed eyelid ( 3 animals), and two of them were in a general poor condition. Except for salivation, these findings were only observed in the individual animals for 1-3 days of the administration period of 28 days. In absence of changes in food consumption and body weight development, these clinical signs were assessed as borderline effects and not indicative of systemic organ toxicity. Relative changes in body weight and food consumption noted upon administration of the test compounds are shown in Table 2. Treated animals did not present any significant changes with respect to body weight when compared to the controls.

Table 2 Relative changes in body weight and food consumption of male and female Crl:Wi(Han) rats $(\mathrm{N}=5$ per group) dosed for 28 days compared to the diet controls. Data were collected on study days 6, 13 and 27 . Treated animals did not present any significant changes $(\mathrm{p}<0.05)$ in body weight compared to the control groups $(\mathrm{N}=10$ per group).

\begin{tabular}{lccccc}
\hline \multirow{2}{*}{ Antibiotic } & \multirow{2}{*}{ Day } & \multicolumn{2}{c}{ Body weight } & \multicolumn{2}{c}{ Food consumption } \\
& & & $\mathbf{f}$ & $\mathbf{~}$ & $\mathbf{f}$ \\
\hline Clindamycin & 6 & 0.97 & 0.99 & 1.30 & 0.80 \\
\cline { 2 - 6 } & 13 & 0.95 & 0.98 & 1.12 & 1.19 \\
\cline { 2 - 6 } & 27 & 0.94 & 1.00 & 0.99 & 1.16 \\
\hline Lincomycin & 6 & 1.00 & 0.99 & 1.00 & 0.99 \\
\cline { 2 - 6 } & 13 & 0.98 & 1.00 & 1.03 & 0.92 \\
\cline { 2 - 6 } & 27 & 0.96 & 1.05 & 1.18 & 0.97 \\
\hline Roxithromycin & 6 & 0.98 & 0.99 & 0.85 & 0.80 \\
\cline { 2 - 6 } & 13 & 0.96 & 1.00 & 0.86 & 0.97 \\
\hline Sparfloxacin & 27 & 0.96 & 1.01 & 1.04 & 1.03 \\
\cline { 2 - 6 } & 13 & 0.96 & 0.98 & 0.94 & 0.77 \\
\hline Streptomycin & 27 & 0.94 & 0.99 & 0.97 & 1.01 \\
\cline { 2 - 6 } & 6 & 0.97 & 0.99 & 1.01 & 0.99 \\
\cline { 2 - 6 } & 13 & 0.96 & 0.98 & 0.92 & 0.91 \\
\hline Vancomycin & 27 & 0.95 & 0.97 & 0.92 & 0.99 \\
\cline { 2 - 5 } & 13 & 0.99 & 0.98 & 0.88 & 0.93 \\
\cline { 2 - 5 } & 27 & 0.97 & 1.00 & 0.93 & 0.92 \\
\hline
\end{tabular}

\section{Bile acid profiling}

The bile acid profiling of feces and plasma samples showed significant changes of primary and secondary, conjugated and unconjugated bile acids in male and female animals (see Table 3). First, a principle component analysis (PCA) was conducted to visualize treatmentrelated effects on the bile acid pool in feces and plasma of male and female animals (see 
Figure 2). For both sexes, the PCA demonstrated that CMC treatment clusters with the control animals in plasma as well as in feces. Roxithromycin and streptomycin were the closest treatment groups to the controls in both matrices showing slight variability compared to the control. Clindamycin and lincomycin, both from the class of lincosamides, with a slight separation between each other but still clustering together, showed the most profound effects on the bile acid pool. In plasma, the main separation was driven by the treatment-related effect of lincomycin. Also, vancomycin and sparfloxacin showed a treatment-related effect and a separation from other classes of antibiotics. Separation in feces was more pronounced than in plasma, indicating a more profound effect of the antibiotics on fecal metabolite composition.

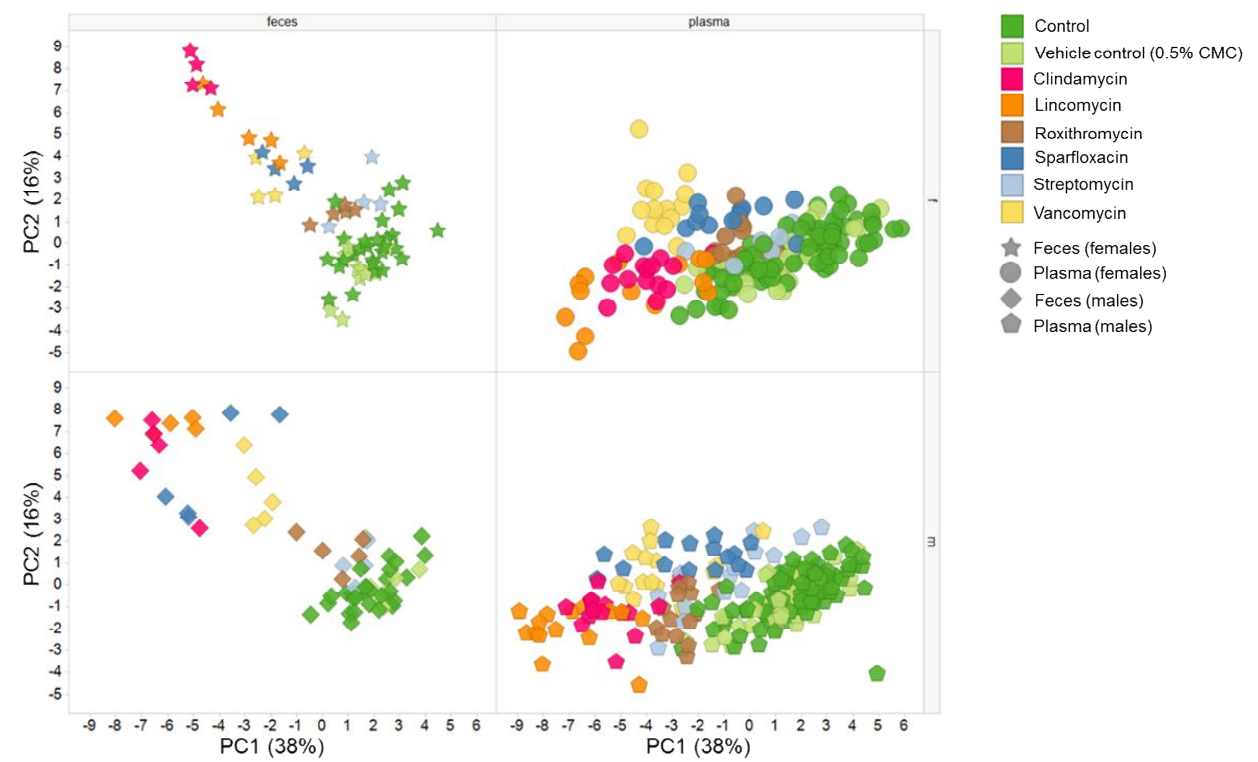

Figure 2 Principal component analysis (PCA) of fecal and plasma metabolic profiles of control and treated female (f) and male (m) animals showing the trellis of results. Animals were dosed daily with antibiotics (clindamycin, lincomycin, vancomycin, streptomycin, roxithromycin, sparfloxacin) and 0.5\% CMC for 28 days. Data shown by the plasma samples contain the metabolite profiles of all three sampling time points (day 7, 14 and 28). Feces were sampled at the end of the study.

The PCA of each day of plasma sampling (day 7, 14 and 28) showed a decrease of variability in PC1 for lincomycin compared to control from day 7 to 28 of lincomycin in males and females (see Supplementary Materials S1 and S2). Further, the bile acid pool in both matrices did not show obvious differences between males and females (see Supplementary Materials S3 and S4). However, the separation of roxithromycin and streptomycin from the control was clearer in male animals, i.e. the treatment-related effect was stronger here.

The hierarchical clustering analysis (HCA) of the bile acid pool in the feces showed a similar pattern as the PCA for male and female animals with a clustering of lincosamides 
(clindamycin and lincomycin) having the most profound effect on the bile acid pool, followed by vancomycin and sparfloxacin, mild effects of streptomycin and roxithromycin, and no effect of CMC (see Figure 3). In plasma samples, the HCA showed in both sexes no or mild effects of the vehicle controls and streptomycin and the most profound effect of the lincosamides antibiotics. In the female animals, roxithromycin clusters with the vehicle control and streptomycin and, as seen in the feces samples, sparfloxacin with vancomycin, whereas roxithromycin showed clusters with the lincosamides antibiotics in the male animals.

Table 3 shows changes in metabolite concentrations relative to the diet controls of primary and secondary bile acids on day 28 in feces and on day 7, 14 and 28 in plasma of antibiotictreated female and male rats. Taurine conjugated primary bile acids, i.e. TCDCA, TCA, $\alpha$ and $\beta$-TMCA, were significantly increased in plasma and feces of antibiotic-treated rats except TCA in the streptomycin group. In contrast, many of the unconjugated and glycine conjugated bile acids, i.e. CDCA, GCDCA, GCA, $\alpha$ - and $\beta$ - MCA, were significantly decreased. 


\section{Chapter 5}

a)

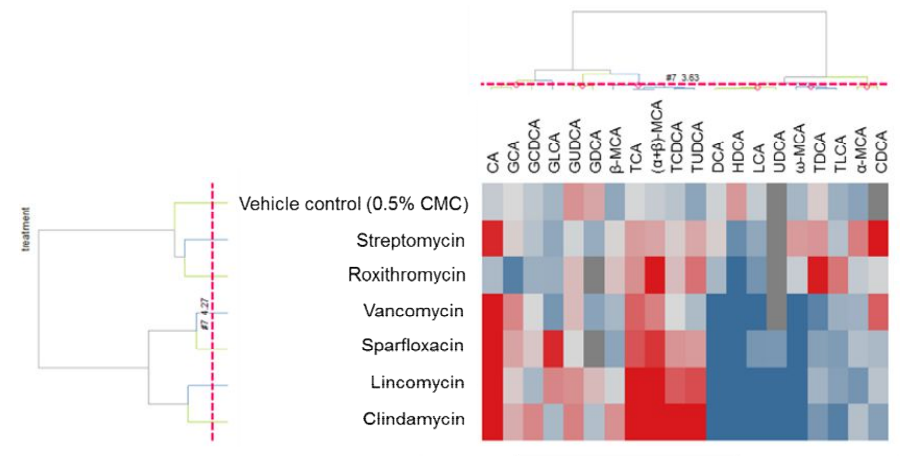

c)

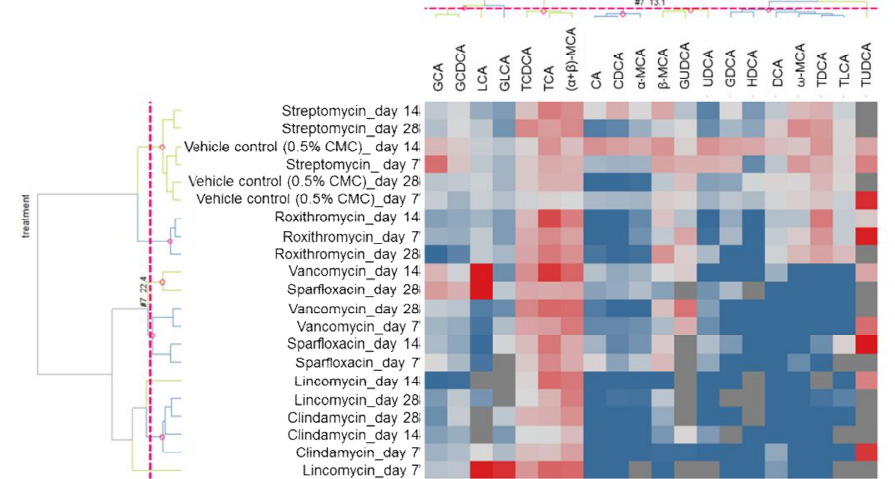

b)

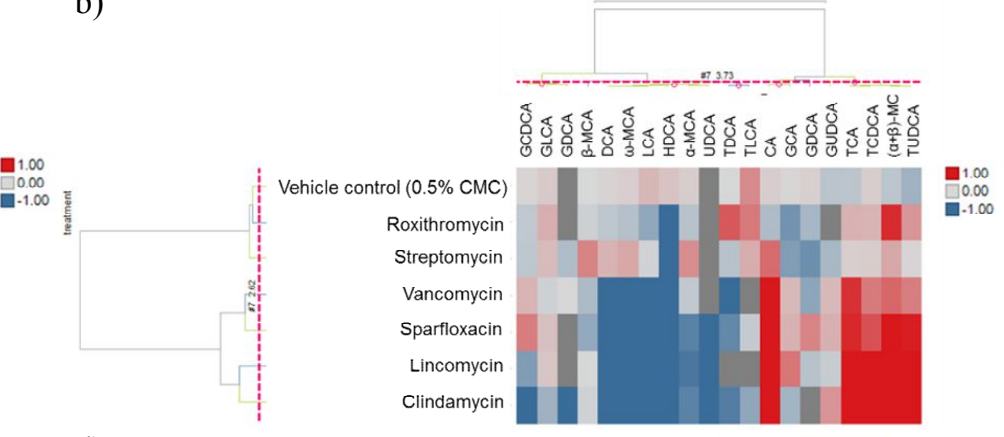

d)

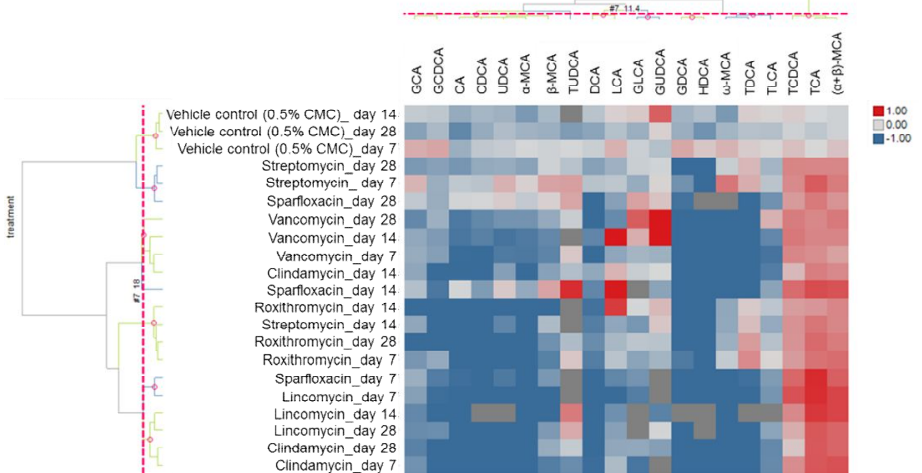

Figure 3 Hierarchical clustering analysis (HCA) of fecal (a and b) and plasma (c and d) metabolic profiles of treated female (a and c) and male (b and d) animals compared to the control animals. The relative abundance (ratio) of bile acids in plasma or feces are log10-transformed. Animals were dosed daily with antibiotics (clindamycin, lincomycin, vancomycin, streptomycin, roxithromycin, sparfloxacin) and $0.5 \% \mathrm{CMC}$ for 28 days. Data shown by the plasma samples contain the metabolite profiles of all three sampling time points (day 7, 14 and 28). Feces were sampled at the end of the study. 
The effect appeared to be more pronounced in male animals. Also, the male group treated with $0.5 \%$ CMC showed a significant decrease in plasma levels on day 14 and 28 of CDCA, CA, $\alpha$ - and $\beta$ - MCA, DCA, MCA and UDCA. However, no significant changes of these bile acids were observed in the feces.

Primary bile acid cholic acid, CA, was highly upregulated in the feces upon antibiotic treatment, with the exception of animals treated with the macrolide antibiotic roxithromycin. Contrarily, plasma levels were significantly downregulated in female animals after lincosamides and roxithromycin treatment. In the other groups, the levels of CA were decreased, however, not significantly. Male animals showed significantly decreased plasma levels of CA in all treatment groups on at least one time point.

With few exceptions, the secondary bile acids DCA, GDCA, TDCA, $\omega$-MCA, HDCA, UDCA, LCA, GLCA and TLCA, were significantly downregulated in plasma and feces of male and female animals. The taurine conjugate TUDCA was significantly upregulated in feces of female and male animals treated with lincosamides and roxithromycin, and in males treated with sparfloxacin. Plasma and feces levels of streptomycin- and roxithromycin-treated animals showed an increase of TDCA, however, not always significantly upregulated. Further, contrary to the other treatments, TLCA was significantly upregulated in feces after roxithromycin treatment.

Solely streptomycin showed an increase, which was significant in the female group, of $\omega$ MCA in feces and plasma. Compared to the other antibiotics treatments, upon streptomycin treatment the secondary bile acids were less affected, except for HDCA.

Vancomycin treatment had a profound effect on the secondary bile acids in samples of males and females; the unconjugated and glycine conjugated primary bile acids were decreased compared to the diet control in male but not in female animals.

The highest values of significant upregulation of the bile acids in both matrices, especially the taurine conjugates, were observed after lincosamide treatment. Here, primary bile acid TMCA in feces was up to 155 -fold increased in females and up to 230 -fold in males. 
Table 3 Metabolite concentration changes relative to the diet controls of primary and secondary bile acids on day 28 in feces (F28) and on day 7, 14 and 28 (P7, P14, P28) in plasma of antibiotic-treated female and male $\mathrm{Crl} / \mathrm{Wi}(\mathrm{Han})$ rats $(\mathrm{N}=5$ per group). Figures in red boxes are significantly increased and those in blue boxes significantly decreased metabolite value changes (Welch $t$ test). The intensity of the color corresponds to the magnitude of the fold change and $\mathrm{p}$-value, bold numbers have a ratio $<0.5$ or $>2$. "NA" indicates that for this analyte, treatment and/or analysis groups there are no contrasts available. Abbreviations: CDCA chenodeoxycholate, GCDCA glycochenodeoxycholate, TCDCA taurochenodeoxycholate, CA cholate, GCA glycocholate, TCA taurocholate, aMCA muricholate (alpha), bMCA muricholate, TMCA(a+b) tauromuricholate acid $(\mathrm{a}+\mathrm{b})$, DCA deoxycholate, GDCA glycodeoxycholate, TDCA taurodeoxycholate, wMCA muricholate, HDCA hyodeoxycholate, UDCA ursodeoxycholate, TUDCA tauroursodeoxycholate, GUDCA glycoursodeoxycholic acid, LCA lithocholate, GLCA glycolithocholic aicd, TLCA taurolithocholic aicd.

\begin{tabular}{|c|c|c|c|c|c|c|c|c|c|c|c|c|c|c|c|c|c|c|c|c|c|c|c|c|}
\hline ratic & & - & $p-V_{i}$ & & & & & & & & & 1 a & & & & & & & & & & & & \\
\hline ratio > & . & & $.01 \leq p$ & -val & - & r & .05 & & ti & $<$ & $n$ & ad & . & . & & - & 0. & .05 & & & & & & \\
\hline ratio > & (1) & $d$ & $.05 \leq 1$ & . & & 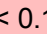 & 10 & & 政 & 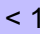 & & & 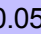 & & & & ) & 10 & & & & & & \\
\hline FEMALES & & ndan & mycin & & ncom & nycin & & Rox & $\begin{array}{r}\text { A } \\
\text { kithro }\end{array}$ & & In & & parfl & loxac & & Stre & eptor & mycin & & anco & omycir & & $\begin{array}{l}\text { Vehi } \\
0.5 \%\end{array}$ & $\begin{array}{l}\text { icle } \\
\text { CMC }\end{array}$ \\
\hline Bile acids & F28 & P7 & P14 P28 & F28 & P7 & P14 F & P28 & F28 & P7 & P14 & P28 & F28 & P7 & P14 & P28 & F28 & P7 & P14 P28 & F28 & P7 & P14 & P28 & F28 P7 & P14 P28 \\
\hline CDCA & 0.3 & 0.0 & \begin{tabular}{|l|l|}
0.0 & 0.0 \\
\end{tabular} & 0.6 & 0.0 & 0.0 & 0.1 & 0.9 & 0.0 & 0.1 & 0.1 & 0.5 & 0.3 & 0.2 & 0.4 & 17.6 & 0.4 & 1.70 .2 & 4.3 & 0.2 & 0.4 & 0.0 & NA 0.5 & 2.00 .1 \\
\hline GCDCA & 2.7 & 0.6 & $\begin{array}{lll}0.4 & 0.8\end{array}$ & 0.5 & 0.7 & 0.1 & 0.7 & 0.4 & 0.4 & 0.4 & 0.2 & 1.3 & 0.5 & 0.3 & 1.6 & 0.6 & 1.3 & 0.91 .0 & 1.0 & 0.4 & 0.9 & 0.6 & 0.70 .4 & $\begin{array}{lll}1.2 & 0.7\end{array}$ \\
\hline TCDCA & 35.0 & 1.4 & 1.01 .5 & 4.5 & 2.3 & 1.1 & 1.0 & 1.4 & 1.7 & 1.5 & 1.8 & 2.3 & 1.2 & 1.4 & 1.8 & 1.2 & 1.2 & $1 . 3 \longdiv { 2 . 6 }$ & 1.0 & 1.9 & 2.4 & 2.6 & 0.60 .9 & 1.11 .2 \\
\hline $\mathrm{CA}$ & 18.6 & 0.0 & 0.00 .0 & 25.0 & 0.0 & 0.0 & 0.1 & 0.5 & 0.0 & 0.0 & 0.1 & 27.4 & 1.1 & 0.5 & 0.3 & 8.3 & 0.5 & 1.00 .1 & 19.5 & 0.2 & 0.6 & 0.1 & 0.70 .5 & 2.30 .1 \\
\hline GCA & 1.7 & 0.4 & \begin{tabular}{|l|l|}
0.3 & 0.2 \\
\end{tabular} & 1.2 & 0.6 & 0.1 & 0.3 & 0.2 & 0.4 & 0.3 & 0.1 & 1.9 & 1.0 & 0.5 & 2.0 & 1.1 & 3.6 & $\begin{array}{lll}0.7 & 0.6\end{array}$ & 2.7 & 0.5 & 1.5 & 0.7 & $\begin{array}{lll}1.0 & 0.8\end{array}$ & $\begin{array}{ll}1.5 & 0.7\end{array}$ \\
\hline TCA & 47.4 & 1.3 & 1.01 .6 & 28.0 & 4.1 & 3.7 & 1.5 & 2.4 & 2.6 & 5.5 & 3.1 & 5.0 & 2.6 & 3.2 & 1.7 & 2.1 & 1.7 & $\begin{array}{lll}3.0 & 2.1\end{array}$ & 4.2 & 2.0 & 7.0 & 3.8 & 1.01 .1 & \begin{tabular}{|l|l|}
2.3 & 1.6
\end{tabular} \\
\hline$\alpha-M C A$ & 0.6 & 0.0 & 0.10 .1 & 0.2 & & 0.0 & 0.1 & 0.7 & 0.2 & 0.2 & 0.1 & 0.6 & 0.7 & 0.2 & 0.6 & 3.0 & 0.4 & 1.00 .4 & 0.4 & 0.2 & 0.4 & 0.1 & 0.30 .5 & 1.70 .1 \\
\hline$\beta-M C A$ & 2.4 & 0.1 & 0.30 .4 & 0.9 & 0.0 & 0.1 & 0.7 & 1.3 & 0.7 & 1.3 & 2.3 & 0.3 & 1.5 & 1.2 & 0.3 & 1.1 & 1.9 & 1.80 .8 & 0.5 & 0.5 & 1.0 & 1.4 & $\begin{array}{lll}0.3 & 1.2\end{array}$ & 2.30 .7 \\
\hline$(\alpha+\beta)-T M C A$ & 155.8 & 2.0 & 1.42 .4 & 90.8 & 3.8 & 3.2 & 2.9 & 10.1 & 1.9 & 2.9 & 2.4 & 7.1 & 1.7 & 1.7 & 1.9 & 1.9 & 1.6 & \begin{tabular}{|l|l|}
2.4 & 2.6 \\
\end{tabular} & 2.9 & 3.1 & 4.0 & 3.6 & 0.81 .0 & 1.41 .6 \\
\hline DCA & 0.0 & 0.4 & $0.0 \quad 0.0$ & 0.0 & 0.3 & 0.0 & 0.1 & 0.5 & 0.8 & 0.6 & 0.9 & 0.0 & 0.1 & 0.0 & 0.1 & 1.1 & 0.7 & 0.71 .2 & 0.0 & 0.0 & 0.4 & 0.0 & 0.90 .9 & 1.41 .1 \\
\hline GDCA & 0.6 & 0.0 & NA 0.1 & 1.5 & 0.1 & 0.0 & NA & NA & 0.5 & 0.4 & 0.1 & NA & 0.1 & 0.3 & 0.5 & 0.4 & 1.5 & $1.0 \mathbf{0 . 5}$ & 0.3 & 0.0 & 0.1 & 0.0 & 1.80 .7 & 1.90 .3 \\
\hline TDCA & 0.2 & 0.0 & \begin{tabular}{|l|l|}
0.0 & 0.0 \\
\end{tabular} & 0.5 & 0.0 & NA & 0.1 & 9.4 & 2.3 & 3.1 & 1.9 & 0.3 & 0.1 & 0.2 & 0.0 & 2.3 & 1.7 & 1.62 .1 & 0.2 & 0.0 & 0.0 & 0.0 & 1.21 .3 & $\begin{array}{ll}2.2 & 1.5\end{array}$ \\
\hline$\omega-M C A$ & 0.1 & 0.0 & \begin{tabular}{|l|l|}
0.0 & 0.0 \\
\end{tabular} & 0.1 & 0.0 & 0.0 & 0.1 & 0.8 & 1.2 & 0.6 & 1.5 & 0.0 & 0.2 & 0.0 & 0.0 & 2.1 & 2.2 & 1.52 .5 & 0.0 & 0.0 & 0.0 & 0.1 & 0.51 .1 & 1.31 .0 \\
\hline HDCA & 0.0 & 0.0 & $0.0 \mathrm{NA}$ & 0.0 & 0.0 & NA & NA & 0.1 & 0.1 & 0.0 & 0.1 & 0.0 & 0.1 & 0.0 & NA & 0.2 & 0.2 & $\begin{array}{lll}0.3 & 0.3\end{array}$ & 0.0 & 0.0 & 0.0 & 0.0 & 2.20 .5 & $\begin{array}{lll}1.8 & 0.9\end{array}$ \\
\hline UDCA & 0.0 & 0.1 & 0.30 .0 & 0.1 & NA & 0.0 & 0.1 & NA & 0.1 & 0.1 & 0.5 & 0.3 & 0.4 & 0.6 & 0.2 & NA & 1.7 & 0.20 .2 & NA & 0.2 & 0.1 & 0.3 & NA 0.4 & \begin{tabular}{|l|l|}
2.3 & 0.3
\end{tabular} \\
\hline TUDCA & 22.6 & 6.9 & NA NA & 5.4 & NA & 2.9 & NA & 3.4 & 9.0 & 1.2 & NA & 2.1 & NA & 10.3 & NA & 1.8 & 2.8 & NA NA & 0.5 & 3.6 & 1.8 & NA & 0.37 .8 & $1.8 \mathrm{NA}$ \\
\hline GUDCA & 2.8 & 0.1 & $1.0 \mathrm{NA}$ & 2.4 & NA & NA & NA & 1.4 & 1.8 & 0.6 & 1.1 & 1.0 & NA & NA & NA & 0.9 & 1.6 & 0.61 .0 & 1.4 & 1.7 & 1.2 & 3.3 & 2.31 .1 & 1.11 .3 \\
\hline LCA & 0.0 & 1.0 & NA NA & 0.0 & 9.5 & NA & 0.2 & 0.2 & 0.7 & 0.5 & 0.7 & 0.2 & 0.1 & 0.0 & 13.1 & 0.4 & 0.7 & $\begin{array}{lll}0.4 & 0.7\end{array}$ & 0.0 & 0.1 & 10.7 & 0.1 & 0.70 .8 & $\begin{array}{ll}0.8 & 0.8\end{array}$ \\
\hline GLCA & 0.5 & 0.5 & $\begin{array}{lll}0.3 & 0.7\end{array}$ & 2.7 & 7.5 & NA & NA & 0.4 & 0.3 & 0.3 & 0.5 & 8.2 & NA & 0.9 & 0.7 & 0.4 & 0.5 & \begin{tabular}{|l|l|}
0.3 & 0.2
\end{tabular} & 0.2 & 0.6 & 0.2 & 0.2 & 0.50 .6 & $0.6 \quad 0.5$ \\
\hline TLCA & 0.1 & 0.0 & NA 0.0 & 0.3 & NA & 0.0 & 0.2 & 3.3 & 0.7 & 0.8 & 1.4 & 0.3 & NA & 1.1 & 0.1 & 0.6 & 1.1 & 0.81 .0 & 0.4 & 0.0 & 0.0 & 0.0 & 0.40 .8 & 1.11 .0 \\
\hline
\end{tabular}

\begin{tabular}{|c|c|c|c|c|c|c|c|c|c|c|c|c|c|c|c|c|c|c|c|c|c|c|c|c|c|c|c|}
\hline \multirow{3}{*}{$\begin{array}{l}\text { MALES } \\
\text { Bile acids }\end{array}$} & \multirow{2}{*}{\multicolumn{4}{|c|}{ Clindamycin }} & \multirow{2}{*}{\multicolumn{4}{|c|}{ Lincomycin }} & \multirow{2}{*}{\multicolumn{4}{|c|}{ Roxithromycin }} & \multirow{2}{*}{\multicolumn{4}{|c|}{ Sparfloxacin }} & \multirow{2}{*}{\multicolumn{4}{|c|}{ Streptomycin }} & \multirow{2}{*}{\multicolumn{4}{|c|}{ Vancomycin }} & \multirow{2}{*}{\multicolumn{3}{|c|}{$0.5 \%$ CMC }} \\
\hline & & & & & & & & & & & & & & & & & & & & & & & & & & & \\
\hline & F28 & P7 & & & F28 & P7 & & P28 & F28 & P7 & & P28 & F28 & $\mathrm{P} 7$ & P14 & P28 & F28 & P7 & $\mathrm{P} 14 \mathrm{~F}$ & & F28 & P7 & P14 & P28 & F28 P7 & & \\
\hline CDCA & & 0.0 & 0.1 & 0 & & 0.0 & & 0. & $\mathrm{NA}$ & 0.0 & 0.0 & 0.1 & NA & 0.0 & 0.3 & & 6 & 0.6 & 0.0 & 0.4 & 1.0 & 0.1 & 0.2 & 0.2 & NA 0.7 & & \\
\hline GCDCA & & 0.1 & 0.1 & 0.1 & 0.3 & 0.1 & 0.1 & & 0.7 & & 0.1 & & 2.9 & 0.1 & & & .7 & 0.4 & 0.1 & 0.3 & 1.5 & 0.3 & 0.3 & 0.4 & & & \\
\hline TCDCA & 13.6 & 2.5 & 1 & 1.2 & 13.4 & 5.0 & 2.6 & & 1.5 & 2.1 & 2.0 & 2.3 & 5.0 & 3.9 & & .1 & .2 & 2.5 & 2.3 & 2.7 & 3.0 & 2.7 & 3.2 & 3.0 & & & \\
\hline CA & .9 & 0.0 & 0.0 & 0.0 & 48.3 & 0.0 & 0.0 & 0.0 & 0.6 & 0.0 & 0.0 & 0.0 & 22.7 & 0.1 & 0.9 & 0.9 & 3.7 & 0.7 & 0.0 & 0.2 & 38.7 & 0.1 & 0.1 & 0.1 & 1.10 .4 & 0.4 & 0.2 \\
\hline GCA & & 0.2 & 0.3 & 0.2 & 3.2 & 0.3 & 0.2 & 0.2 & 0.2 & 0.2 & 0.1 & 0.1 & 1.6 & 0.4 & 0.6 & 0.7 & .3 & 1.5 & 0.3 & 0.3 & 1.4 & 0.4 & 0.4 & 1.2 & & & \\
\hline ה & 0.8 & 4.7 & & 4.6 & 62.0 & & & & 1.6 & & & & 8.3 & 8.0 & & & 1.1 & 4.5 & & 2.7 & 7.4 & & 2.7 & & & & \\
\hline$\alpha-M C A$ & 0.2 & 0.0 & 0.0 & 0.0 & 0.1 & 0.0 & 0.0 & 0.0 & 0.6 & 0.1 & 0.0 & 0.1 & 0.2 & 0.0 & 0.4 & 0.7 & 2.4 & 1.1 & 0.0 & 0.3 & 0.7 & 0.1 & 0.1 & 0.2 & 0.9 & 0. & 0.5 \\
\hline$\beta-\mathrm{MCA}$ & 0.9 & 0.1 & 0.3 & 0.4 & 1.0 & 0.1 & 0.1 & 0.3 & 0.8 & 0.2 & 0.0 & 0.2 & 0.4 & 0.3 & 1.9 & 1.1 & 2.8 & 1.9 & 0.5 & 0.9 & 0.5 & 0.2 & 0.2 & 0.4 & 0.9 & & 0.4 \\
\hline$(\alpha+\beta)-$ & 92.7 & 5.0 & 2.6 & 4.0 & 227.9 & 5.9 & 5.9 & 3.9 & 8.0 & 3.6 & 2.7 & 3.0 & 16.9 & 4.2 & 5.0 & 2.3 & 1.8 & 3.0 & 2.6 & 2.6 & 3.9 & 1.9 & 2.7 & 3.1 & 0 & & 0.9 \\
\hline DCA & 0.0 & 0.1 & & 0.0 & 0.0 & 0.0 & 0.0 & 0.0 & 0.7 & 0.1 & 0.1 & 0.2 & 0.0 & 0.0 & 0.2 & 0.0 & 1.6 & 0.0 & 0.1 & 0.3 & 0.0 & 0.0 & 0.2 & 0.0 & & 0.0 & 0.3 \\
\hline GDCA & & 0.0 & 0 & & 6 & 0.0 & & & 0.5 & 0.1 & 0.0 & 0.1 & 2.7 & 0.0 & 1.0 & & .2 & 0.2 & 0.1 & 1 & 0.3 & 0.0 & 0.0 & .1 & & & \\
\hline TDCA & 0.1 & 0.0 & & 0.0 & NA & 0.0 & NA & & 4.6 & 2.5 & 1.2 & 2.1 & 0.2 & 0.1 & 0.0 & & 0.6 & 17 & 1.4 & 1.5 & 0.1 & & 0.0 & 0.0 & & & \\
\hline$\omega-M C$ & 0 & 0.0 & 0.0 & 0.0 & 0.0 & 0.0 & 0.0 & 0.0 & 0.6 & 0.7 & 0.4 & 0.4 & 0.0 & 0.0 & 0.0 & NA & 1.8 & 3.2 & 0.4 & 0.8 & 0.0 & & 0.0 & 0.0 & & 0. & \\
\hline $\mathrm{HDCA}$ & & 0.0 & & 0.0 & .0 & 0.0 & NA & NA & 0.0 & 0.0 & 0.0 & 0.0 & 0.0 & 0.0 & 0 & A & 0.0 & 0.0 & 0.0 & .0 & 0.0 & 0.0 & 0.0 & 1.0 & & & .2 \\
\hline UDCA & 0.0 & 0.0 & 0.3 & 0.0 & 0.0 & 0.0 & NA & 0.0 & NA & 0.0 & $0.1 \mathrm{C}$ & 0.0 & 0.0 & 0.1 & 1.2 & 1.2 & NA & 1.6 & 0.2 & 0.4 & NA & 0.1 & 0.1 & 0.2 & NA 0.8 & & \\
\hline TUDCA & 28.3 & 0.2 & 0.6 & 0.7 & 29.0 & NA & 3.0 & 1.7 & 2.2 & 1.2 & & 0.6 & 9.3 & NA & 7.8 & 1.8 & 1.0 & 2.0 & NA & 0.6 & 2.9 & 1.2 & NA & 0.8 & 0.40 .9 & & \\
\hline GUDCA & 2.3 & NA & & 0.3 & 0.8 & NA & $0 ?$ & & NA & 0.5 & & 0.6 & 1.6 & NA & & 1.3 & 0.5 & 1.1 & 1.2 & 0.9 & 1.4 & 0.4 & 16.3 & 10.1 & & 4.2 & \\
\hline LCA & & 0.5 & & 0.3 & 0.0 & & 0.2 & & 0.3 & & & 0.5 & 0.0 & 0.2 & & 0.3 & 0.9 & 0.7 & 0.3 & 4 & 0.1 & & 28.0 & 0.2 & & & \\
\hline GLCA & & 0.1 & 0.8 & 0.4 & 1.3 & 0.1 & NA & NA & 1.6 & 0.3 & 0.9 & 0.7 & 1.3 & 0.2 & NA & 1.9 & 1.4 & 0.4 & 0.2 & 0.8 & 0.9 & 0.5 & 1.6 & 4.4 & 1.20 .8 & 1.2 & 0.4 \\
\hline TLCA & 0.3 & 0.2 & 0.1 & 0.2 & NA & 0.2 & NA & 0.3 & 3.1 & 1.1 & 0.7 & 0.6 & 0.7 & 0.3 & 0.3 & 0.2 & 1.7 & 0.7 & 0.4 & 0.5 & $\mathrm{NA}$ & 0.1 & 0.2 & 1.6 & 2.51 .1 & 1.1 & 0.6 \\
\hline
\end{tabular}




\section{Discussion}

Upon treatment with antibiotics possessing different activity spectra and bioavailabilities, feces and plasma samples showed significant changes of primary and secondary, conjugated and unconjugated bile acids in male and female animals. Whereas lincosamides, roxithromycin and sparfloxacin are bioavailable, streptomycin is poorly and vancomycin non-bioavailable after oral administration (Arimori et al., 1998; Brown and Riviere, 1991; Derrick and Reilly, 1983; Geraci et al., 1956; Musther et al., 2014).

The PCA and HCA show clear treatment-related effects with no effects of the vehicle CMC clustering with control animals, slight effects of streptomycin and roxithromycin, followed by clear effects of vancomycin and sparfloxacin and strongest effects of the lincosamides antibiotics. However, in the HCA plasma samples showed differences between genders in the clustering of antibiotics. In the female animals, roxithromycin showed a slight effect as observed in the feces samples, whereas it showed a similar pattern and clustering with the lincosamides antibiotics.

As in our last study, we observed significant changes of the primary bile acids TCA and CA in both plasma and feces (Behr et al., 2018). In general, it was shown that in rat plasma the majority of conjugated bile acids are taurine derivates (García-Cañaveras et al., 2012). We hypothesized that the antibiotics-induced loss of microbes possessing specific bile salt hydrolases that deconjugate taurine conjugates could account for the strikingly higher values of taurocholic acid observed in both plasma and feces, with the exception of the streptomycin group. Bacteroidetes exhibit taurocholic acid-specific bile salt hydrolases (BSHs) (Ridlon et al., 2006) and the depletion of these microbes was consistent with the high levels of the conjugated taurocholic acid. Further, taurocholic acid is transported actively from the intestine and it was shown that taurocholic acid uptake is increased in the ileal epithelium of GF rats (Riottot and Sacquet, 1985). The antibiotic treatment could have led to an increased reabsorption and eventually in higher levels of this metabolite in plasma. Other taurine conjugated bile acids, TCDCA, and $\alpha$ - and $\beta$-TMCA, were significantly increased in plasma and feces of antibiotic-treated rats except for the streptomycin group, which will be discussed below. In a study with germ-free mice, cytochrome P450 expression and activity associated with bile acid metabolism and drug detoxification mechanisms were analyzed in liver microsomes during a colonization process of these mice (Claus et al., 2011). The analysis revealed the involvement of gut microbiota in CYP8b1 induction and the consecutive regulation of the TCA/TMCA ratio. Also, significant overexpression of nuclear receptors was observed at the end of the colonization process which implies that the gut microbiome plays an important role in the endogenous xenobiotic detoxification pathways. Primary bile acid CA was highly upregulated in the feces, with the exception of animals treated with the macrolide antibiotic roxithromycin. Cholic acid is not actively transported and inefficiently absorbed by passive diffusion and could have accumulated in the intestine due to the loss of microorganisms with a $7 \alpha-$ dehydroxylation activity which would have transformed CA to DCA. Contrary to the feces, many treatments caused downregulated levels of CA in plasma, as well as many of the 
unconjugated and glycine conjugated bile acids, i.e. CDCA, GCDCA, GCA, $\alpha$ - and $\beta$ MCA, were significantly decreased. There seemed to be slight sex differences since the effect was more pronounced in male animals. Firmicutes are able to deconjugate both taurine and glycine, which was in line with the decreased levels of glycochenodeoxycholic acid in feces, probably due to the lack of Firmicutes. Unconjugated bile acids have a central role in the metabolic homeostasis of the gastrointestinal tract and can activate nuclear receptors as FXR, PXR, CAR, VDR and TGR5 (Huttenhower et al., 2012; Hylemon et al., 2009; Thomas et al., 2008). Furthermore, they are in general important for controlling the microbial population and the integrity of the intestinal barrier function (Jia et al., 2018; Vavassori et al., 2009).

Secondary bile acids and their conjugates DCA, GDCA, TDCA, $\omega$-MCA, HDCA, UDCA, LCA, GLCA and TLCA were significantly downregulated in plasma and feces of male and female animals due to the loss of intestinal bacteria which transform the primary into secondary bile acids by unique microbial enzymes. For example, the bile salt hydrolase (BSH) cleaves the peptide linkage between the amino acid and the primary bile acid. Furthermore, mainly anaerobic bacteria possess a $7 \alpha$ - dehydroxylation activity to convert primary into secondary bile acids (Ridlon et al., 2006). However, again the taurine conjugated bile acid TUDCA accumulated in feces of female and male animals treated with lincosamides and roxithromycin, and in males treated with sparfloxacin. In the female group, TUDCA was also upregulated, however, not significantly. Important to note here is that there are indications that UDCA and its conjugated derivates can be produced by the liver of mice and could therefore be considered primary bile acids (Selwyn et al., 2015).

Regarding the overall alteration of the bile acid pool in plasma and feces, streptomycin and roxithromycin appeared to differ from the other treatments. Streptomycin, a poorly bioavailable aminoglycoside, has in contrast to the other antibiotics poor activity against obligate anaerobic and most gram-positive bacteria. However, the majority of the intestinal microbes belongs to the obligate anaerobes. Contrary to the other treatments, TLCA, in high levels considered as a promoter of colon cancer (Farhana et al., 2016), was significantly upregulated in feces after roxithromycin treatment. Similar results for macrolides compared to other classes of antibiotics were observed in a study with rats ( $\mathrm{Li}$ et al., 2017). Interestingly, compared to other macrolide antibiotics, roxithromycin and its main metabolite were reported to exhibit a very poor affinity for rat liver cytochrome P-450 even at high doses (i.e. 500 and 1,000 mg/kg p.o.) and a decreased ability to induce liver cytochrome P-450 PCNE, an isozyme implicated in drug associations involving some macrolide antibiotics (Delaforge et al., 1988). Therefore, it could be assumed that roxithromycin did not interfere with liver cytochromes and xenobiotic metabolisms and that consequently unique changes in the bile acid pool are due to changes of the gut microbiome.

Vancomycin, which is used for the treatment of severe Clostridium difficile infections (CDI), showed a profound effect on the secondary bile acids. Vancomycin treatment is reported to deplete microbial members responsible for converting primary bile acids into 
secondary bile acid which reduces the resistance to Clostridium difficile colonization (Theriot et al., 2015). It is a non-bioavailable antibiotic, i.e. changes in the bile acid pool were highly likely induced by changes of the gut microbial composition or the loss of bile acid-metabolizing microbes.

In a recent study, the vehicle control $0.5 \% \mathrm{CMC}$ showed no significant changes in the microbial community of the rat feces (Behr et al., 2018). Moreover, in this study, the bile acid profiling showed also no significant changes in the feces of both sexes and in plasma of females. However, the male group treated with $\mathrm{CMC}$ showed a slight but significant decrease in plasma on two-time points of CDCA, CA, $\alpha$ - and $\beta$ - MCA, DCA, MCA and UDCA. In the literature, there are only few studies on $\mathrm{CMC}$ and its potential effect on the bile acid pool. Anderson et al. reported an increase of bile acids in the feces of human volunteers consuming $15 \mathrm{~g} \mathrm{CMC}$ daily for 23 days but no changes in plasma biochemistry (Anderson et al., 1986). In another study broiler chickens fed CMC diet excreted more bile acids in the feces and the total aerobic and anaerobic microbial counts small intestine significantly increased (Smits et al., 1998). The authors hypothesized that CMC decreases apparent lipid digestibility by reducing the concentration of bile acids in the chyme which may further contribute to malabsorption of lipids. Since feces were shown to be a more sensitive matrix for bile acid changes caused by antibiotics than plasma, it could have been expected that such changes should have also shown up in the feces. Nevertheless, if CMC would have had an exclusive effect in the upper parts of the intestinal tract, then this may have been unnoticed in the present study. Further studies on the impact of CMC on the gut microbiome and bile acid alterations in the small intestine should be performed since no changes in the feces could be observed.

Summarizing, this study shows that antibiotics have a profound impact on the bile acid metabolism via alterations of the intestinal microbiota - in particular shown by the poorly or non-bioavailable antibiotics streptomycin and vancomycin - and potential feedback mechanisms regarding the bile acid production in the liver. The impact of the antibiotic treatment was stronger in the feces indicating a clearer and more profound effect of the antibiotics on fecal metabolite composition. Although different classes of antibiotics with different activity spectra against gut microbes were applied, they induced an increase of taurine-conjugated primary bile acids in both plasma and feces. Contrary, cholic acid and most of the secondary bile acids were significantly downregulated in plasma whereas cholic acid accumulated in the feces. This accumulation was not seen for roxithromycin indicating a different mode of action for the macrolide antibiotic.

Not only could changes in the gut microbiome lead to alterations of the bile acid pool (Jia et al., 2018) but also the bile acids themselves can influence the composition of the microbiota (Ridlon et al., 2014). Bile acids are essential to make compounds (natural and xenobiotic) more bioavailable, and hence increase absorption. An alteration of the bile acid pool, therefore, is highly likely to have an effect on bioavailability and absorption and this could potentially lead to altered plasma concentrations of both endogenous metabolites, the metabolome, as well as xenobiotics. Additionally, bile acids themselves interact with 
nuclear receptors (Makishima et al., 2002; Maruyama et al., 2002; Xie et al., 2001). Here too, an alteration in the concentration of bile acids is expected to have an effect in terms of reduced or increased gene activation. This may impact the cytochrome P450 expression and could interfere with drug detoxification mechanisms, finally leading to either a detoxification or toxification of a compound of interest. Regarding the points mentioned above changes of the bile acid pool might have implications for affecting the gut-liver axis, the immune system and other systems of the body.

Acknowledgement: We would like to thank Ms Antje Behr for the technical illustration of the bile acid figure and Ms Irmgard Weber for her skillful assistance in taking plasma samples.

Funding information: The authors have no relevant affiliations or financial involvement with any organization or entity with a financial interest in or financial conflict with the subject matter or materials discussed in the manuscript. This includes employment, consultancies, honoraria, stock ownership or options, expert testimony, grants or patents received or pending, or royalties. No writing assistance was utilized in the production of this manuscript.

\section{Appendix A. Supplementary data}

Supplementary data to this article can be found online at https://doi.org/10.1016/j.taap.2018.11.012. 


\section{REFERENCES}

Anderson, D.M.W., Eastwood, M.A., Brydon, W.G., 1986. The dietary effects of sodium carboxymethylcellulose in man. Top. Catal. 1, 37-44. https://doi.org/10.1016/S0268$005 X(86) 80005-4$

Arimori, K., Miyamoto, S., Fukuda, K., Nakamura, C., Nakano, M., 1998. Characteristic difference in gastrointestinal excretion of clarithromycin and roxithromycin. Biopharm. Drug Dispos. 19, 433-438. https://doi.org/10.1002/(SICI)1099081X(199810)19:7<433::AID-BDD122>3.0.CO;2-Z

Behr, C., Ramírez-Hincapié, S., Cameron, H.J., Strauss, V., Walk, T., Herold, M., Beekmann, K., Rietjens, I.M.C.M., van Ravenzwaay, B., 2018. Impact of lincosamides antibiotics on the composition of the rat gut microbiota and the metabolite profile of plasma and feces. Toxicol. Lett. 296, 139-151. https://doi.org/10.1016/j.toxlet.2018.08.002

Brown, S.A., Riviere, J.E., 1991. Comparative pharmacokinetics of amionoglycoside antibiotics. J. Vet. Pharmacol. Ther. 14, 1-35. https://doi.org/10.3109/14992027.2010.524253

Claus, S.P., Ellero, S.L., Berger, B., Krause, L., Bruttin, A., Molina, J., Paris, A., Want, E.J., Waziers, I. De, Cloarec, O., Richards, S.E., Wang, Y., Dumas, M., Ross, A., Rezzi, S., Kochhar, S., Bladeren, P. Van, Lindon, J.C., Holmes, E., Nicholson, J.K., 2011. Colonization-Induced Host-Gut Microbial Metabolic Interaction. MBio 2, e00271-10. https://doi.org/10.1128/mBio.00271-10.Editor

Delaforge, M., Sartori, E., Mansuy, D., 1988. In vivo and in vitro effects of a new macrolide antibiotic roxithromycin on rat liver cytochrome P-450: Comparison with troleandomycin and erythromycin. Chem. Biol. Interact. 68, 179-188.

Derrick, C.W., Reilly, K.M., 1983. Erythromycin, lincomycin, and clindamycin. Pediatr. Clin. North Am. 30, 63-69. https://doi.org/10.1016/S0031-3955(16)34320-6

Dior, M., Delagrèverie, H., Duboc, H., Jouet, P., Coffin, B., Brot, L., Humbert, L., Trugnan, G., Seksik, P., Sokol, H., Rainteau, D., Sabate, J.M., 2016. Interplay between bile acid metabolism and microbiota in irritable bowel syndrome. Neurogastroenterol. Motil. 28, 1330-1340. https://doi.org/10.1111/nmo.12829

Duboc, H., Rajca, S., Rainteau, D., Benarous, D., Maubert, M.A., Quervain, E., Thomas, G., Barbu, V., Humbert, L., Despras, G., Bridonneau, C., Dumetz, F., Grill, J.P., Masliah, J., Beaugerie, L., Cosnes, J., Chazouillères, O., Poupon, R., Wolf, C., Mallet, J.M., Langella, P., Trugnan, G., Sokol, H., Seksik, P., 2013. Connecting dysbiosis, bile-acid dysmetabolism and Gut inflammation in inflammatory bowel diseases. Gut 62, 531-539. https://doi.org/10.1136/gutjnl-2012-302578

Farhana, L., Nangia-Makker, P., Arbit, E., Shango, K., Sarkar, S., Mahmud, H., Hadden, T., Yu, Y., Majumdar, A.P.N., 2016. Bile acid: a potential inducer of colon cancer stem cells. Stem Cell Res. Ther. 7, 181. https://doi.org/10.1186/s13287-016-0439-4

García-Cañaveras, J.C., Donato, M.T., Castell, J. V., Lahoz, A., 2012. Targeted profiling of circulating and hepatic bile acids in human, mouse, and rat using a UPLC-MRM-MSvalidated method. J. Lipid Res. 53, 2231-2241. https://doi.org/10.1194/jlr.D028803

Geraci, J., Heilman, F., Nichols, D., Wellman, E., Ross, G., 1956. Some laboratory and 
clinical experiences with a new antibiotic, vancomycin. Antibiot Annu. 90-106.

Gray, J.E., Purmalis, A., Feenstra, E.S., 1964. Animal Toxicity Studies of a New Antibiotic, Lincomycin. Toxicol Appl Pharmacol 6, 476-496.

Gray, J.E., Purmalis, A., Mulvihill, W.J., 1966. Further toxicologic studies of lincomycin. Toxicol. Appl. Pharmacol. 9, 445-454. https://doi.org/10.1016/0041-008X(66)900032

Gray, J.E., Purmalis, A., Purmalis, B., Mathews, J., 1971. Ultrastructural studies of the hepatic changes brought about by clindamycin and erythromycin in animals. Toxicol. Appl. Pharmacol. 19, 217-233. https://doi.org/10.1016/0041-008X(71)90108-6

Gray, J.E., Weaver, J.A., Feenstra, E.S., 1972. The Oral Toxicity of Clindamycin in Laboratory Animals. Toxicol Appl Pharmacol 21, 516-531.

Ho, K.J., Xiong, L., Hubert, N.J., Nadimpalli, A., Wun, K., Chang, E.B., Kibbe, M.R., 2015. Vancomycin treatment and butyrate supplementation modulate gut microbe composition and severity of neointimal hyperplasia after arterial injury. Physiol. Rep. 3, 1-12. https://doi.org/10.14814/phy2.12627

Huttenhower, C., Gevers, D., Knight, R., Al., E., 2012. Structure, function and diversity of the healthy human microbiome. Nature 486, 207-214. https://doi.org/10.1038/nature11234

Hylemon, P.B., Zhou, H., Pandak, W.M., Ren, S., Gil, G., Dent, P., 2009. Bile acids as regulatory molecules. J. Lipid Res. 50, 1509-1520. https://doi.org/10.1194/jlr.R900007-JLR200

Jia, W., Xie, G., Jia, W., 2018. Bile acid-microbiota cross-talk in gastrointestinal inflammation and carcinogenesis. Nat Rev Gastroenterol Hepatol 15, 111-128. https://doi.org/10.1016/j.jhsa.2009.09.008.Validity

Kamp, H., Strauss, V., Wiemer, J., Leibold, E., Walk, T., Mellert, W., Looser, R., Prokoudine, A., Fabian, E., Krennrich, G., Herold, M., Ravenzwaay, B. van, 2012. Reproducibility and robustness of metabolome analysis in rat plasma of 28-day repeated dose toxicity studies. Toxicol. Lett. 215, 143-149.

Khurana, S., Raufman, J.P., Pallone, T.L., 2011. Bile acids regulate cardiovascular function. Clin. Transl. Sci. 4, 210-218. https://doi.org/10.1111/j.17528062.2011.00272.x

Li, Y., Hafey, M.J., Duong, H., Evers, R., Cheon, K., Holder, D.J., GalijatovicIdrizbegovic, A., Sistare, F.D., Glaab, W.E., 2017. Antibiotic-induced elevations of plasma bile acids in rats independent of Bsep inhibition. Toxicol. Sci. 157, 30-40. https://doi.org/10.1093/toxsci/kfx015

Long, S.L., Gahan, C.G.M., Joyce, S.A., 2017. Interactions between gut bacteria and bile in health and disease. Mol. Aspects Med. 56, 54-65. https://doi.org/10.1016/j.mam.2017.06.002

Makishima, M., Lu, T.T., Xie, W., Whitfield, G.K., Domoto, H., Evans, R.M., Haussler, M.R., Mangelsdorf, D.J., 2002. Vitamin D receptor as an intestinal bile acid sensor. Science (80-. ). 296, 1313-1316. https://doi.org/10.1126/science.1070477

Maruyama, T., Miyamoto, Y., Nakamura, T., Tamai, Y., Okada, H., Sugiyama, E., Nakamura, T., Itadani, H., Tanaka, K., 2002. Identification of membrane-type 
receptor for bile acids (M-BAR). Biochem. Biophys. Res. Commun. 298, 714-719. https://doi.org/10.1016/S0006-291X(02)02550-0

Mouzaki, M., Wang, A.Y., Bandsma, R., Comelli, E.M., Arendt, B.M., Zhang, L., Fung, S., Fischer, S.E., McGilvray, I.G., Allard, J.P., 2016. Bile acids and dysbiosis in nonalcoholic fatty liver disease. PLoS One 11, 1-13. https://doi.org/10.1371/journal.pone.0151829

Musther, H., Olivares-Morales, A., Hatley, O.J.D., Liu, B., Rostami Hodjegan, A., 2014. Animal versus human oral drug bioavailability: Do they correlate? Eur. J. Pharm. Sci. 57, 280-291. https://doi.org/10.1016/j.ejps.2013.08.018

Pereira-Fantini, P.M., Bines, J.E., Lapthorne, S., Fouhy, F., Scurr, M., Cotter, P.D., Gahan, C.G.M., Joyce, S.A., 2016. Short bowel syndrome (SBS)-associated alterations within the gut-liver axis evolve early and persist long-term in the piglet model of short bowel syndrome. J. Gastroenterol. Hepatol. 31, 1946-1955. https://doi.org/10.1111/jgh.13383

Ridlon, J., Kang, D., Hylemon, P., Bajaj, J., 2014. Bile acids and the Gut Microbiome. Curr. Opin. Gastroenterol. 332-338. https://doi.org/10.1097/MOG.0000000000000057

Ridlon, J.M., Kang, D.-J., Hylemon, P.B., 2006. Bile salt biotransformations by human intestinal bacteria. J. Lipid Res. 47, 241-259. https://doi.org/10.1194/jlr.R500013JLR200

Riottot, M., Sacquet, E., 1985. Increase in the ileal absorption rate of sodium taurocholate in germ-free or conventional rats given an amylomaize-starch diet. Br. J. Nutr. 53, 307-310. https://doi.org/10.1079/BJN19850038

Selwyn, F.P., Csanaky, I.L., Zhang, Y., Klaassen, C.D., 2015. Importance of Large Intestine in Regulating Bile Acids and GLP-1 in Germ-free Mice. Drug Metab. Dispos. 5, 1544-1556. https://doi.org/10.1124/dmd.115.065276

Slopianka, M., Herrmann, A., Pavkovic, M., Ellinger-Ziegelbauer, H., Ernst, R., Mally, A., Keck, M., Riefke, B., 2017. Quantitative targeted bile acid profiling as new markers for DILI in a model of methapyrilene-induced liver injury in rats. Toxicology 386, 110. https://doi.org/10.1016/j.tox.2017.05.009

Smits, C.H.M., Veldman, A., Verkade, H.J., Beynen, A.C., 1998. The Inhibitory Effect of Carboxymethylcellulose with High Viscosity on Lipid Absorption in Broiler Chickens Coincides with Reduced Bile Salt Concentration and Raised Microbial Numbers in the Small Intestine. Poult. Sci. 77, 1534-1539. https://doi.org/10.1093/ps/77.10.1534

Strauss, V., Mellert, W., Wiemer, J., Leibold, E., Kamp, H., Walk, T., Looser, R., Prokoudine, a., Fabian, E., Krennrich, G., Herold, M., Van Ravenzwaay, B., 2012. Increased toxicity when fibrates and statins are administered in combination - A metabolomics approach with rats. Toxicol. Lett. 211, 187-200. https://doi.org/10.1016/j.toxlet.2012.03.798

Theriot, C.M., Bowman, A. a, Young, V.B., 2015. Antibiotic-Induced Alterations of the Gut Microbiota Alter Secondary Bile Acid Production and Allow for Clostridium difficile Spore Germination and Outgrowth in the Large Intestine. mSphere 1, e00045-15. https://doi.org/10.1128/mSphere.00045-15.Editor 
Thomas, C., Pellicciari, R., Pruzanski, M., Auwerx, J., Schoonjans, K., 2008. Targeting bile-acid signalling for metabolic diseases. Nat. Rev. Drug Discov. https://doi.org/10.1038/nrd2619

van Ravenzwaay, B., Coelho-Palermo Cunha, G., Strauss, V., Wiemer, J., Leibold, E., Kamp, H., Walk, T., Mellert, W., Looser, R., Prokoudine, a., Fabian, E., Krennrich, G., Herold, M., 2010. The individual and combined metabolite profiles (metabolomics) of dibutylphthalate and di(2-ethylhexyl)phthalate following a 28-day dietary exposure in rats. Toxicol. Lett. 198, 159-170. https://doi.org/10.1016/j.toxlet.2010.06.009

van Ravenzwaay, B., Cunha, G.C.P., Leibold, E., Looser, R., Mellert, W., Prokoudine, a., Walk, T., Wiemer, J., 2007. The use of metabolomics for the discovery of new biomarkers of effect. Toxicol. Lett. 172, 21-28. https://doi.org/10.1016/j.toxlet.2007.05.021

van Ravenzwaay, B., Herold, M., Kamp, H., Kapp, M.D., Fabian, E., Looser, R., Krennrich, G., Mellert, W., Prokoudine, A., Strauss, V., Walk, T., Wiemer, J., 2012. Metabolomics: A tool for early detection of toxicological effects and an opportunity for biology based grouping of chemicals-From QSAR to QBAR. Mutat. Res. - Genet. Toxicol. Environ. Mutagen. 146, 144-150. https://doi.org/10.1016/j.mrgentox.2012.01.006

van Ravenzwaay, B., Kamp, H., Montoya-parra, G.A., Strauss, V., Fabian, E., Mellert, W., Walk, T., Peter, E., Looser, R., Herold, M., 2016. The development of a database for metabolomics - looking back on ten years of experience. Int. J. Biotechnol. 14, 4768. https://doi.org/10.1504/IJBT.2015.074801

Vavassori, P., Mencarelli, A., Renga, B., Distrutti, E., Fiorucci, S., 2009. The Bile Acid Receptor FXR Is a Modulator of Intestinal Innate Immunity. J. Immunol. 183, 62516261. https://doi.org/10.4049/jimmunol.0803978

Villa, P., Sassella, D., Corada, M., Bartosek, I., 1988. Toxicity, uptake, and subcellular distribution in rat hepatocytes of roxithromycin, a new semisynthetic macrolide, and erythromycin base. Antimicrob. Agents Chemother. 32, 1541-1546.

von Keutz, E., Schlüter, G., 1999. Preclinical safety evaluation of moxifloxacin, a novel fluoroquinolone. J. Antimicrob. Chemother. 43, 91-100. https://doi.org/10.1093/jac/43.suppl_2.91

World Health Organization, 1997. WHO FOOD ADDITIVES SERIES 39 [WWW Document]. URL http://www.inchem.org/documents/jecfa/jecmono/v39je05.htm (accessed 10.16.18).

Xie, W., Radominska-Pandya, A., Shi, Y., Simon, C.M., Nelson, M.C., Ong, E.S., Waxman, D.J., Evans, R.M., 2001. An essential role for nuclear receptors SXR/PXR in detoxification of cholestatic bile acids. Proc. Natl. Acad. Sci. 98, 3375-3380. https://doi.org/10.1073/pnas.051014398

Zou, S., Fang, L., Lee, M.-H., 2017. Dysbiosis of gut microbiota in promoting the development of colorectal cancer. Gastroenterol. Rep. 6, 1-12. https://doi.org/10.1093/gastro/gox031 


\section{Chapter 6}

General discussion 


\section{OVERVIEW OF THE RESULTS AND MAIN FINDINGS}

The gut microbiome plays an important role in health and disease of the host, and can be influenced by factors such as diet, the host condition, environmental chemical exposure and drugs [1-4]. In the last years, omics technologies as metagenomics, metaproteomics and metabolomics shown to be promising tools towards a mechanistic understanding of the gut microbiome, providing a holistic view of the host and its microbiome. Integrative approaches that not only asses the composition of the gut microbiome but also its functionality have been implemented to unravel the complex dynamics of host-microbiome interactions [5]. Compared with metagenomic approaches, which face the difficulty of high intra- and interspecies variabilities regarding the composition of the microbiome, a metabolome analysis offers the advantage to elucidate the functionality, in terms of gene activity and metabolic output of the microbes, with a lower variability [6-8]. In this mechanistically focused project, the aim was to elucidate interactions between gut microbes and the host by applying metabolomics and taxonomic profiling, thereby assessing functional microbial changes and the consequences for the host organism.

Chapter 2 presented the first investigation of the mammalian-microbiome co-metabolism of endogenous metabolites by analyzing the plasma of antibiotic-treated Wistar rats. To identify plasma metabolites related to microbiome changes due to antibiotic treatment, broad-spectrum antibiotics belonging to the class of aminoglycosides (neomycin, gentamicin), fluoroquinolones (moxifloxacin, levofloxacin) and tetracyclines (doxycycline, tetracycline) were applied. These were administered orally for 28 days to male rats, and blood was sampled for metabolic profiling after 7, 14 and 28 days. Whereas aminoglycosides are poorly bioavailable after oral administration, the other two classes can be absorbed from the gut [9-13]. For each class of antibiotics specific plasma metabolome patterns could be established in the MetaMap ${ }^{\circledR}$ Tox database and first key metabolites (hippuric acid, indole-3-acetic acid, indole-3-propionic acid, 3-indoxylsulfate and glycerol) could be identified, indicating a change of the gut microbiome. However, for the tetracycline and fluoroquinolone antibiotics, a possible influence of systemic toxicity had to be taken into account when evaluating their effects on the plasma metabolome due to their bioavailability.

With this study design it could be shown that gut microbial changes lead to changes in the plasma metabolome, therefore further experimental studies were set up to elucidate this interaction. Consequently, three antibiotics (vancomycin, streptomycin and roxithromycin) from different classes were administered orally to rats with a subsequent metabolic profiling in feces, cecum content and gut tissue (jejunum, ileum, cecum, colon and rectum) analyzing the same set of metabolites as before in plasma (Chapter 3). After antibiotic treatment, metabolite changes observed at different dose levels and in both sexes were evaluated. Treatment-related effects could be observed in the metabolite profile of feces and cecum content, but not in the different gut tissues. The most relevant changes in metabolite values were comparable in feces and cecum content and also among sexes. The metabolite profile showed compound specific effects on the microbiome, in line with the 
activity spectra of the antibiotics tested. Vancomycin showed the largest effects in the feces and cecum metabolome, followed by roxithromycin and then by streptomycin for which changes were modest. For all antibiotics the largest changes were observed for the classes of lipids, bile acids, amino acids and amino acid related metabolites. In general, it has to be noted that analysis was targeted. An untargeted analysis or a broader range of metabolites, e.g. including the analysis of short chain fatty acids (SCFAs), may have revealed significant differences between matrices and possibly even sexes.

With our targeted analysis, we identified feces as the best matrix for further investigations regarding an assessment of metabolic effects of new compounds with antibiotic activity. Thus, it offers the advantage of being a non-invasive sampling method, also enabling a longitudinal study design. However, this matrix only partially reflects the metabolite profile of the gut content, since the upper part, i.e. the small intestine, shows distinct differences in the composition of the microbial composition [14] which may considerably differ regarding the metabolite profile. For our metabolome studies, it requires overnight-fasted animals for the blood sampling and then the animals do not have content in the small intestine, so this matrix was not included in the studies.

In Chapter 4, we applied lincomycin and clindamycin, another class of antibiotics known to show no or low systemic toxicity after oral administration, and modulated microbial communities of Wistar rats to gain a comprehensive understanding of the implications of microbiome alterations. A metabolomics approach and taxonomic profiling were applied to characterize the effects of these antibiotics on the functionality and composition of the microbiome and to identify microbiome-related metabolites. After treatment, the diversity of the microbial community was drastically reduced. Whereas other phyla disappeared, the abundance of Firmicutes and Proteobacteria was highly increased. Again, most changes in plasma and feces metabolites were observed for metabolites belonging to the class of complex lipids, fatty acids and related metabolites as well as amino acids and related compounds. The three analyzed primary bile acids (taurocholic acid, glycochenodeoxycholic acid and cholic acid) were markedly affected and displayed diverging results. In both plasma and feces taurocholic acid was highly upregulated upon treatment, whereas glycochenodeoxycholic acid was downregulated. Interestingly, cholic acid was upregulated in feces, however, it was downregulated in plasma. Taking into account that quantitatively the most pronounced changes were related to bile acids, it can be stated that the gut microbiome plays a very important role in the composition of the bile acid pool because secondary bile acids are exclusively formed by bacterial enzymes.

Since a change of the gut microbiota can impact the bile acid pool, we used six antibiotics from five different classes (lincosamides, glycopeptides, macrolides, fluoroquinolones, aminoglycosides) and modulated microbial communities of Wistar rats to elucidate changes in the bile acid metabolism related to gut microbial changes (Chapter 5). The results showed that changes in the gut microbial community affected the bile acid pool in plasma and feces of the host, and that bile acid profiling can be indicative for an alteration of the gut microbiome. After treatment, significant changes of primary and secondary bile acids in 
both matrices of treated animals could be observed. For cholic acid and taurocholic acid, as discussed in Chapter 4, there was an increase of taurine-conjugated primary bile acids in both plasma and feces. Contrary, cholic acid and most of the analyzed secondary bile acids were found to be significantly downregulated in plasma whereas cholic acid accumulated in the feces. Although different classes of antibiotics with different activity spectra against gut microbes were applied, the overall effect on the bile acid pool tended to be similar in both matrices. However, streptomycin showed less significant changes, probably because it is not active against obligate anaerobes. Since the bile acid-liver-gut microbiota axis plays an important role in the host's health, e.g. via activation or inactivation of nuclear receptors in the liver or intestine, an alteration of the bile acid pool might have implications for toxicological evaluations regarding the gut-liver axis, the immune system and other body functions known to be influenced by the gut microbiota and needs to be further investigated. 


\section{GENERAL DISCUSSION AND FUTURE PERSPECTIVES}

In this project, the aim was to obtain detailed insight in the mammalian-microbiome cometabolism of endogenous metabolites and the extent to which the microbiome influences the plasma metabolome observed with the help of the MetaMap ${ }^{\circledR}$ Tox database. To elucidate this interaction, antibiotics as well as vehicle and diet controls were used to modulate the microbial communities of Wistar-rats. After 28-day oral administration, metabolomic analyses of plasma, gut tissue and content (feces and cecum-content) were performed. Additionally, DNA was extracted from rat feces and the $16 \mathrm{~S}$ subunit was sequenced to perform a core diversity analysis. In the following, the applied methods and their results, as well as their limitations and suggestions for improvements are pointed out. The techniques used, i.e. metabolomics and taxonomic profiling, are discussed in more depth and bridged to statistical evaluations and metagenomic databases. The last section then elucidates the xenobiotic metabolic capacity of the gut microbiome, gives future perspectives for research and closes with a conclusion.

\section{Metabolomics, key metabolites and (systemic) effect of antibiotics}

Within the context of the omics technology, metabolomics is the analysis of small endogenous molecules such as carbohydrates, amino acids, nucleic acids or fatty acids and their derivatives resulting from biochemical pathways [15]. These metabolites, in its entirety called the metabolome, can be measured in biological samples, for example, urine or plasma as a matrix. Compared to other omics technologies, metabolomics, is from a statistical perspective, more powerful in detecting robust effects [16] and the use of sensitive LC-MS and GC-MS techniques offers the possibility to detect a broad range of metabolites. In the past years it has been shown that metabolomics can serve as a powerful tool in risk assessment, e.g. as a read-across tool, and in toxicological research $[17,18]$. Besides the targeted analysis of metabolites used here, there is also the possibility to perform an untargeted analysis. This does not provide quantitative results but normalized peak height of the metabolites can be considered semi-quantitative [19]. The advantage is that with the untargeted method, relative changes of metabolites in samples can be readily revealed which would lead to important findings. In contrast to the untargeted method, with our targeted analysis we analyzed about 200 metabolites in feces and plasma and identified a range of key metabolites indicating a change of the gut microbiome (Chapter 2-5). However, to depict a more precise picture of gut microbial changes, and with this of interindividual or sex-specific effects, the following classes of metabolites may be useful to gain new insights and could be additionally measured: short chain fatty acids (SCFAs), choline metabolites, phenolic, benzoyl and phenyl derivates, polyphenols and flavonoids, vitamins, and polyamines [20-27]. Other valuable analytes might be dietary carbohydrates and complex glycoconjugates since they are more and more playing a role in the research of how to maintain or restore a healthy gut microbiome [28].

The metabolome data in the MetaMap ${ }^{\circledR}$ Tox database is based on blood sampling and subsequent metabolic profiling of more than 200 metabolites after 7, 14 and 28 days of test 
substance treatment in a 28-day toxicity study in rats. To use metabolomics in toxicology as a screening tool, various metabolite patterns for different toxicological targets and mode of actions (MoAs) were established in the MetaMap ${ }^{\circledR}$ Tox database, with the aim to identify and predict the toxicological MoA of new test substances, e.g. in the liver, kidney, thyroid, testes, blood, nervous system and endocrine system [29]. In other words, this means that changes of the endogenous metabolites, derived from the organs affected by a compound, are analyzed. In the last years, however, research has demonstrated that gut microbiomederived metabolites and co-metabolites can be found in the host's blood [25]. Hence, the challenge of this project was that oral administration of a bioavailable substance can cause changes in both endogenous, and gut microbiome-derived or -associated metabolites in plasma. After antibiotic treatment with tetracyclines, fluoroquinolones, macrolides and lincosamides, which are absorbed from the gastrointestinal tract, a possible influence of systemic toxicity had to be taken into account when evaluating their effects on the plasma metabolome (Chapters 2, 4, 5). Dose levels used in these studies were carefully chosen, to avoid systemic toxicity, while being high enough to induce an effect on the microbiota. For these reasons, it is assumed that the plasma metabolome changes caused by these antibiotics in our studies, in the absence of effects on body weight development or clinical signs over a longer period of time, were primarily the result of their effect on the microbial functionality. To strengthen the correctness of pure microbiome-related metabolite identification, we applied a) aminoglycosides and vancomycin because these antibiotics are poorly or not absorbed at all from gut, and b) performed a metabolic profiling in different matrices of the gut to analyze the same metabolites as before in plasma in the immediate surrounding matrix of the bacteria. We observed that the metabolite profile was not only dependent on the bioavailability and the potential systemic effect but also on the bactericidal/-static efficacy spectrum of the antibiotics. These factors led to specific changes in the metabolite patterns which could be clearly observed when evaluating the metabolome of the gut content (Chapter 3). Still, using these differences of the metabolite patterns is an opportunity to identify and characterize substances that may have antibiotic properties and affect specific phyla or species of the gut microbiome.

In Chapter 2, three metabolites, of which relative abundances in plasma from male rats were decreased after antibiotic treatment in all patterns (tetracyclines, fluoroquinolones, aminoglycosides), could be identified as key metabolites: indole-3-acetic acid (IAA), hippuric acid and glycerol. Since these metabolites could be found in the aminoglycoside pattern, it is highly likely that these metabolites are derived from the microbiota. Indole-3acetic acid, a protein-bound uremic solute, is generated either from bacterial indole production or from dietary tryptophan degradation by both endogenous and bacterial cells, e.g. Clostridium sporogenes, Clostridium bartlettii and Escherichia coli [30]. In antibiotictreated rats, the tryptophan metabolism appeared to be significantly altered because levels of indole-containing substances like 3-indoxylsulfate and IAA were decreased both in urine and feces whereas tryptophan and tryptamine levels were increased significantly in feces 
[22]. Likewise, after lincosamides treatment, indole derivatives were significantly altered in plasma (Chapter 4).

Another significantly decreased key metabolite in plasma, hippuric acid (HA), is produced by the microbes via benzoate by metabolizing aromatic compounds and polyphenols. Benzoate is then conjugated with glycine in the liver, kidney and intestine by bacteria as Clostridium difficile, F. prausnitzii, Bifidobacterium, Subdoligranulum, Lactobacillus [3134]. Bacterial suppression by antibiotics reduces the excretion of hippuric acid in blood, urine and feces of rodents $[21,22,24]$ and in germ-free animals the plasma and urinary concentrations of hippuric acid were found to be reduced $[25,26]$. We could not analyze HA in the feces, however, benzoate levels in the surrounding gut tissue as well as in the gut content did not show any significant changes. Furthermore, the plasma metabolic profiles of many antibiotic-treated animals showed that the amino acid glycine was present in lower concentrations compared to the controls and conversely significantly upregulated in the feces. This indicates that glycine, more likely as a consequence of bacterial impairment, could not be further metabolized. However, this does not explain the accumulation in the intestine. Thus, we suggest that the significant decrease of hippuric acid in plasma is not due to the lack of benzoic acid but rather to a decrease of glycine in the host's system. Given the importance and the wide range of metabolic pathways, e.g. the glutamatergic system, in which glycine is involved, a change in the concentration of this amino acid could have consequences for the host's well-being [35].

Besides significant changes of amino acids and related metabolites, complex lipids, fatty acids and related metabolites including glycerol were generally affected in both feces and plasma, also depending on the class of antibiotics (Chapter 2-5). All three primary bile acids, which were measured in the metabolome analysis, showed profound alterations after antibiotic treatment (Chapter 4). Therefore, an additional method was set up to analyze a set of 20 bile acids in plasma and feces of antibiotic-treated rats (Chapter 5). Since an altered bile acid pool may affect the absorption or excretion of (endogenous) metabolites or xenobiotics, or induce changes in activation or inactivation of nuclear receptors in the liver or intestine, this could lead to altered plasma concentrations, a detoxification or toxification of xenobiotics, or as a secondary effect to a change of endogenous metabolites, the metabolome, e.g. in plasma. The changes of plasma or feces levels of certain metabolites, reflecting the regulation of tricacylglycerols and other fatty acids, due to changes in the bile acid pool were described in Chapter 3 and 4. As presented in Chapter 5, all tested antibiotics had a profound impact on the bile acid metabolism via alterations of the intestinal microbiota - in particular shown for the poorly or non-bioavailable antibiotics streptomycin and vancomycin - and potential feedback mechanisms regarding the bile acid production in the liver. All five classes of antibiotics induced an increase of taurineconjugated primary bile acids in both plasma and feces, and a significant downregulation of secondary bile acids. Interestingly, cholic acid was downregulated in plasma but accumulated in the feces after antibiotic treatment, with the exception of roxithromycin which indicates a different mode of action for this macrolide antibiotic. Since cholic acid 
accumulated in the intestine, it can be hypothesized that it could not be further converted into secondary bile acids due to the loss of microorganisms with a $7 \alpha$-dehydroxylation activity. It can be assumed that since cholic acid is not actively transported and in general inefficiently absorbed by passive diffusion, it accumulates in the gut as seen for other fatty acids like the tricacylglycerols. In general, it was shown that the majority of conjugated bile acids in rat plasma are taurine derivatives [36]. If the liver produces more taurineconjugates, which accumulated in plasma and feces, in relation to cholic acid, then this could account for the significantly lower plasma values of cholic acid. Summarizing, the bile acids demonstrated to be valuable key metabolites regarding changes of the gut microbial communities. Moreover, the bile acids reflect the tight interaction between microbial metabolism and host, directly affecting for example nuclear receptors in the liver which play a very important role in the field of pharmacology and toxicology.

As mentioned above, we performed a metabolic profiling in different matrices of the gut to analyze the same metabolites, as before in plasma, in the immediate surrounding matrix of the bacteria (Chapter 3). Whereas the metabolite profiles of sampled gut matrices (i.e. jejunum, ileum, rinsed cecum, colon, rectum) did not show treatment-related effects, feces and cecum content of treated animals had significantly different metabolome profiles compared to the control. As no clear changes between feces and cecum content could be observed in the overall metabolite profile, thus indicating the potential to profit from having a non-invasive sampling method, we analyzed feces samples for all subsequent projects. Furthermore, it was shown that the metabolite profile can be correlated with changes in the gut microbial community analyzed in this matrix and comparisons could be drawn with in vitro incubations which are normally based on feces samples. Recently, in a study examining more than 1000 metabolites in human feces from 786 individuals from a population-based twin study, the fecal metabolome was found to be only modestly influenced by host genetics but largely reflected the gut microbial composition [37]. In our studies, compared to plasma, feces were shown to be a more sensitive matrix for metabolome changes caused by antibiotics and thus it can be expected that changes should first show up in the feces. Interestingly, in our targeted analysis, feces and cecum content from male and female animals showed a similar metabolite profile. This was somewhat surprising as for plasma metabolite profiles, it is well known that sex has a profound impact and in general significant differences between sexes, also in untreated controls are observed [38]. However, in the current analysis of the feces metabolome, steroids and catecholamines were not measured and these analyte levels could be different between the sexes. Nonetheless, the very similar metabolite profiles - containing about 200 metabolites led to the conclusion that there are no significant differences between sexes regarding the gut microbial communities. This finding will be discussed below where the microbial communities of male and female animals will be compared.

In general, the possibility that potential systemic toxicity can occur after oral treatment aggravates the distinction between endogenous and microbiome-derived or -associated 
metabolites and had to be taken to account when evaluating the metabolome. The long-term effect of many antibiotics on the host or even the mode of action on the host's cells are not well-known or at least not yet proven. For example, the US Food and Drug Administration (FDA) announced in 2008 severe side effects of fluoroquinolone antibiotics as tendon rupture and irreversible nerve damage, and reported a few years later the fluoroquinoloneassociated disability (FQAD) [39]. Since mitochondria show some similarities to bacteria, mitochondrial toxicity may affect the mammalian cells but in the meantime also epigenetic changes seem to play a role $[40,41]$. Short-term and long-term consequences of antibiotic use on the human microbiome are now an evolving field of research related to diseases such as malnutrition, obesity, diabetes, infection, and even a short-term treatment is associated to have adverse effects on the host's health [42]. Yet, in modern industrial animal farming, low doses of antibiotics are used to enhance growth and prevent diseases, and this practice is considered to be, in part, responsible for drug resistance of human pathogenic bacteria, thereby increasing the epidemic risks. The mechanisms behind the growth promoting properties of antibiotics are still not well-known and remain to be (urgently) elucidated which would enable the targeted development of alternatives to substitute antibiotics.

\section{Taxonomic profiling}

The analysis of ribosomal RNA gene sequences yields a description of a microbial community in terms of species richness, structure, composition, and members of microbial communities [43]. To assess the effects of the antibiotics, possessing different activity spectra, on the gut microbial composition, a taxonomic profiling was done. Therefore, a $16 \mathrm{~S}$ rRNA profiling and an amplicon sequencing were performed to characterize the taxonomic structure of a microbial community. Compared to a metagenomic approach, e.g. shotgun metagenomics that aims to characterize whole genomes, the analysis of the $16 \mathrm{~S}$ rRNA gene provides a relatively rapid and cost-effective method for assessing the bacterial diversity and abundance. However, the 16S rRNA profiling entails limitations which are discussed in the following.

The community analysis performed in this project delivered qualitative data, i.e. the obtained abundance of microbial taxa or operational taxonomic units (OTUs) is a relative value of the sample sequence library rather than an absolute value of the total bacteria cell count. If microbial cell numbers vary considerably between samples, the relative profiling might have implications for correlation analyses of, e.g. bacterial species with (semi)quantitative data such as metabolome data [44]. Quantitative microbiome profiling (QMP) of bacterial loads can be assessed by quantitative polymerase chain reaction (qPCR), downsized to an even sampling depth, or flow cytometry, and defined as the ratio between sample size and microbial load, as described by Vandeputte et al. to provide more power in assessing variation within and between individuals [45]. It was shown that correlation analyses built upon quantitative data increased the detection of true bacterial associations (sensitivity) and decreased the false positive correlation rates. As compared to our studies, the differences between the individuals, or a healthy versus a patient cohort, are expected to 
be more subtle than the differences in microbial communities of antibiotic-treated versus non-treated animals. Bender et al. showed that the relative abundance has a stronger impact on variability than does absolute biomass, suggesting that it is feasible to detect differences in bacterial populations in very low-biomass samples [46]. Still, for a subsequent correlation analysis of our results between microbial and metabolite changes, this potential confounder has to be taken into account.

Another confounder in the analysis of $16 \mathrm{~S}$ rRNA data is $16 \mathrm{~S}$ gene copy number (GCNs). Since different bacteria contain different numbers of GCNs, sequence variant counts are biased towards clades with a higher number of GCNs [47]. Louca et al. systemically evaluated the predictability of public sequenced genome data sets and recommended against correcting for $16 \mathrm{~S}$ GCNs in microbiome analyses unless certain requirements are fulfilled [47]. However, regarding our results of the rat gut microbiome, many operational taxonomic units (OTUs) are still not assigned in the databases. Thus, correcting for $16 \mathrm{~S}$ GCNs would probably lead to a higher bias in the results than when they would be analysed without $16 \mathrm{~S}$ GCNs as it was performed.

Others suggest, due to the reduction of phylogenetic resolution by the limited read length of second-generation sequencing platforms, the enrichment of 16S rRNA genes from metagenomic samples with a hybridization capture approach [48]. Further, high-throughput methods for assessing community structure in terms of biomass are lacking. Kleiner et al. showed with metaproteomics-based methods that assessment of species biomass contributions adds an important dimension to the analysis of microbial community structure [49]. They assessed the microbial community structure using protein abundance as a measure for biomass contributions of individual populations and observed high variabilities in results when comparing the metaproteomics approach and 16S rRNA gene amplicon sequencing [49].

In general, with potentially conflicting associations of clinical outcomes, regarding for example genomic and functional predictions, it was demonstrated how important the experimental design and execution on reproducibility and robustness of microbiome studies is [46,50]. Technical and methodological variation, for example due to different sample storage, DNA extraction protocols, primer choice, library preparation, and laboratory conditions have a great impact on metagenomic analyses and thus on the inferred microbial composition [50-53]. In a recent review of Knight et al., the best practices for performing a microbiome study are discussed, including experimental design, choice of molecular analysis technology, methods for data analysis and the integration of multiple omics data sets [54]. The authors inter alia suggest to replace operational taxonomic unit-based analyses with new methods that are based on exact sequence variants and methods for integrating metagenomic and metabolomic data [54]. Summarizing, the standardization of protocols for microbiome studies is essential to minimize biases in the results in order to enable the comparison of data between different studies and to allow drawing clear conclusions about the role of the microbiome in health and disease. 
To characterize the changes in the gut microbiota composition, 16S rRNA gene sequencing was performed from the isolated bacterial DNA in feces taken from Wistar rats on day 28 of the studies. Figure 1 shows a principal coordinate analysis (PCoA) of weighted UniFrac distances of the gut microbial community of female and male rats after treatment with different antibiotics (vancomycin, streptomycin, sparfloxacin, roxithromycin, lincomycin and clindamycin), a restricted diet control, vehicle controls (carboxymethylcellulose (CMC, $0.5 \%$ ) and corn oil), and the control groups of the four independent studies. A clear treatment-related effect could be observed for all antibiotics except streptomycin, which shows a weak separation, on the gut microbiome and the main separation on Axis 1 was driven by the treatment.
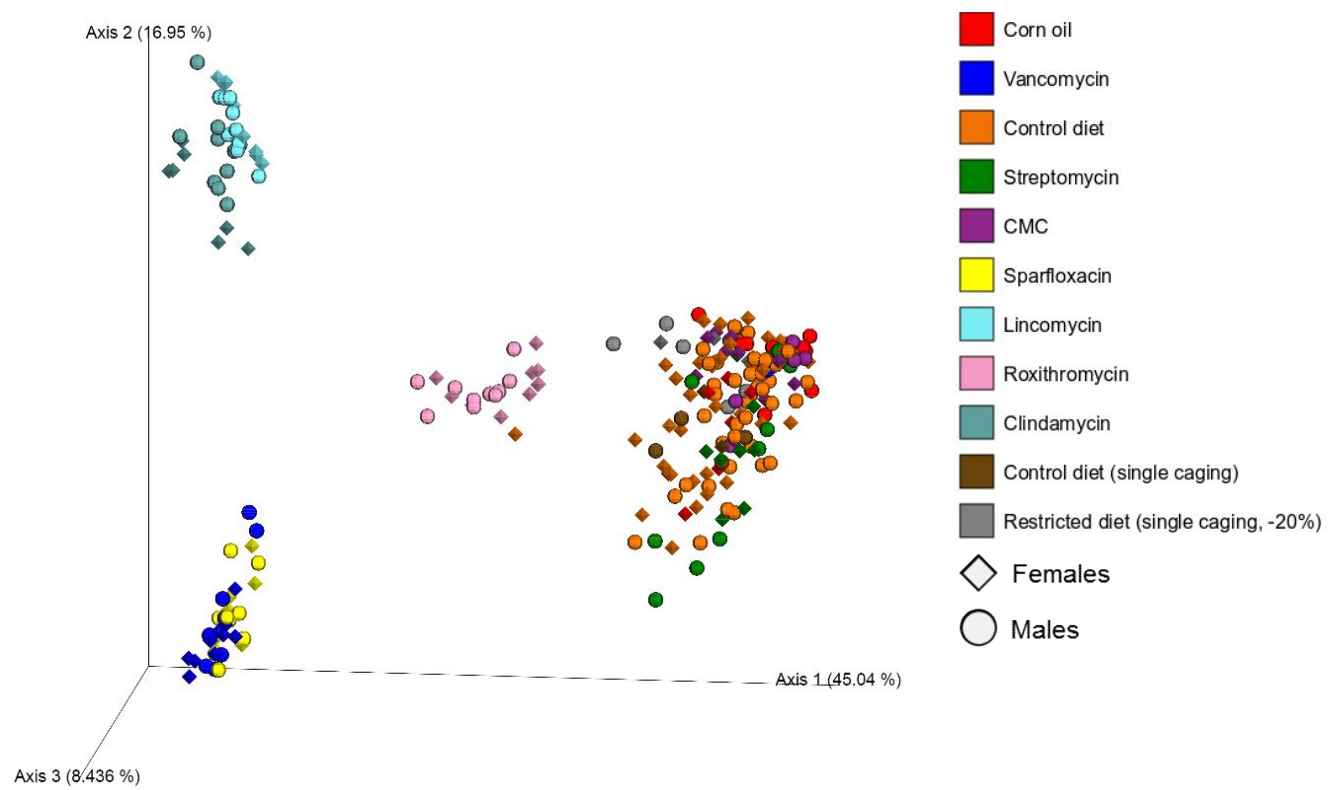

Figure 1 Principal Coordinate Analysis (PCoA) of the gut microbial community showing the treatment-related effect of antibiotics applied for the studies (vancomycin, streptomycin, sparfloxacin, roxithromycin, lincomycin and clindamycin) versus the restricted diet control, the vehicles carboxymethylcellulose (CMC, 0.5\%) and corn oil, and the control groups in the gut microbial composition of male and female animals. Feces samples were taken on day 28 of the studies, bacterial DNA was extracted and the 16S ribosomal RNA gene was sequenced. PCoA was calculated using weighted UniFrac distance on the denoised dataset obtained from the dada2 workflow.

As already seen in the metabolome (Chapters 3 and 5), the effect of streptomycin was not as pronounced as the effects of the other antibiotics. Streptomycin is not active against obligate anaerobe bacteria, i.e. the majority of the intestinal microbes were not affected which was reflected in the taxonomic and metabolomic results. An analysis of both the gut microbial community and the metabolome from the small intestinal content would probably show clear alterations compared to control animals. Furthermore, changes in the bile acid 
pool could be more distinct since the active uptake of $95 \%$ of the bile acids takes place in the ileum which is the connection between small and large intestine.

Community structures of control groups, vehicle controls and restricted diet controls clustered together, as well as lincomycin and clindamycin from the lincosamides antibiotics (Chapter 4) and surprisingly vancomycin, a glycopeptide drug, and sparfloxacin, a fluoroquinolone drug. Since vancomycin is not bioavailable after oral administration but sparfloaxcin is, a comparison of the feces and plasma metabolome as well as an analysis if metabolites and specific genera correlate, could help to identify more microbiome-derived or -associated metabolites and to gain new insights into metabolic pathways.

In general, community structures of both male and female animals clustered together. Interestingly, the same observation was made when comparing the metabolome of cecum content and feces (Chapter 3). Only for animals treated with streptomycin, sex differences were observed, especially in cecum content. In females, lipids and triacylglycerols were stronger increased compared to males. As mentioned above, an analysis of the small intestine where lipid and bile acid metabolism predominates could reveal a sex-specific reaction as shown in a study of Markle et al., were sex-specific differences in the composition of the microbiome of cecum content were found at puberty and were most apparent in adult mice [55]. Therefore, the differences of both alpha and beta diversity were assessed for a deeper analysis of sex-specific effects between control and treated groups. Alpha diversity describes the within-sample diversity using the OTUs whereas the beta diversity calculates a pairwise sample dissimilarity and represents either a similarity or a distance between the samples. As described in Chapter 4, alpha diversity and effective species number was calculated for each sample by taking the exponential of ShannonWiener index [56], and for the effective species number Kruskal-Wallis statistics were calculated using the diversity plugin of QIIME2. The Kruskal-Wallis $(\mathrm{H})$ test is a nonparametric test that determines if there are statistically significant differences between two or more groups. This statistical test was ran to test for differences in effective species which is a measure of species diversity that accounts for the evenness and number of species where the $H$ value is essentially a measure of effect size. Usually, the $H$ value is reported with the $\mathrm{p} / \mathrm{q}$ values. The $\mathrm{q}$-value is the $\mathrm{p}$-value after correcting for multiple testing to reduce the chance of false positives. Table 1 shows the differences in alpha diversity of female versus male animals treated with vehicles (CMC and corn oil), antibiotics (roxithromycin, streptomycin, vancomycin, lincomycin and clindamycin) and control animals (diet control and 20\% reduced diet). A minor significant difference after testing using Kruskal- Wallis was only seen for the roxithromycin group and the control group with single caging. 
Table 1 Differences in alpha diversity of female versus male animals treated with vehicles (CMC and corn oil), antibiotics (roxithromycin, streptomycin, vancomycin, lincomycin and clindamycin) and control animals (diet control and 20\% reduced diet). Effective species number (Chao et al., 2010) was calculated for each sample by taking the exponential of Shannon-Weiner index and Kruskal-Wallis statistics were calculated using the diversity plugin of QIIME2. The last column indicates where the p value is $<0.05(*)$ or $<0.01(* *)$.

\begin{tabular}{|c|c|c|c|c|c|}
\hline Females & Males & $\mathbf{H}$ & p-value & q-value & Significance \\
\hline Vehicle control $(0.5 \% \mathrm{CMC})$ & Vehicle control $(0.5 \% \mathrm{CMC})$ & 2.940 & 0.086 & 0.115 & \\
\hline Diet control (study 1) & Diet control (study 1) & 1.651 & 0.199 & 0.249 & \\
\hline Roxithromycin & Roxithromycin & 3.863 & 0.049 & 0.068 & * \\
\hline Streptomycin & Streptomycin & 0.167 & 0.683 & 0.731 & \\
\hline Vancomycin & Vancomycin & 0.966 & 0.326 & 0.384 & \\
\hline Diet control (study 2) & Diet control (study 2) & 0.334 & 0.248 & 0.304 & \\
\hline Vehicle control (corn oil) & Vehicle control (corn oil) & 1.776 & 0.183 & 0.232 & \\
\hline Lincomycin & Lincomycin & 1.120 & 0.290 & 0.347 & \\
\hline Sparfloxacin & Sparfloxacin & 1.421 & 0.233 & 0.287 & \\
\hline Diet control (single caging) & Diet control (single caging) & 3.938 & 0.047 & 0.066 & * \\
\hline Diet control (study 3) & Diet control (study 3) & 0.060 & 0.806 & 0.833 & \\
\hline Restricted diet (20\%) & Restricted diet $(20 \%)$ & 1.320 & 0.251 & 0.307 & \\
\hline Clindamycin & Clindamycin & 3.810 & 0.051 & 0.070 & \\
\hline Diet control (study 4) & Diet control (study 4) & 2.063 & 0.151 & 0.193 & \\
\hline
\end{tabular}

Table 2 shows the PERMANOVA analysis [57] of weighted UniFrac distance for the beta diversity which was performed using the diversity plugin in QIIME2. Pseudo F values compare the total sum of squared dissimilarities (or ranked dissimilarities) among objects belonging to different groups to that of objects belonging to the same group [58]. Larger Fratios indicate more pronounced group separation, however, the significance of this ratio is usually of more interest than its magnitude [58].

Table 2 Pairwise PERMANOVA results from QIIME2 beta group significance. Diet control animals of study 1, 2 and 4 and the vehicle controls (CMC and corn oil) show a minor, yet significant, difference between male and female animals, as well as roxithromycin and clindamycin. The last column indicates where the $\mathrm{p}$ value is $<0.05$ $(*)$ or $<0.01(* *)$.

\begin{tabular}{|c|c|c|c|c|c|c|c|}
\hline Females & Males & Sample Size & Permutations & pseudo-F & p-value & q-value & Significance \\
\hline Vehicle control $(0.5 \% \mathrm{CMC})$ & Vehicle control $(0.5 \% \mathrm{CMC})$ & 19 & 999 & 4.867 & 0.001 & 0.001 & $* *$ \\
\hline Diet control (study 1) & Diet control (study 1) & 20 & 999 & 2.715 & 0.001 & 0.001 & ** \\
\hline Roxithromycin & Roxithromycin & 20 & 999 & 3.487 & 0.018 & 0.020 & * \\
\hline Streptomycin & Streptomycin & 19 & 999 & 1.643 & 0.125 & 0.129 & \\
\hline Vancomycin & Vancomycin & 20 & 999 & 1.481 & 0.071 & 0.075 & \\
\hline Diet control (study 2) & Diet control (study 2) & 18 & 999 & 2.536 & 0.012 & 0.014 & * \\
\hline Vehicle control (corn oil) & Vehicle control (corn oil) & 18 & 999 & 3.443 & 0.001 & 0.001 & ** \\
\hline Lincomycin & Lincomycin & 20 & 999 & 1.356 & 0.246 & 0.249 & \\
\hline Sparfloxacin & Sparfloxacin & 18 & 999 & 1.336 & 0.270 & 0.271 & \\
\hline Diet control (single caging) & Diet control (single caging) & 10 & 999 & 1.316 & 0.174 & 0.179 & \\
\hline Diet control (study 3) & Diet control (study 3) & 19 & 999 & 1.447 & 0.145 & 0.150 & \\
\hline Restricted diet $(20 \%)$ & Restricted diet $(20 \%)$ & 10 & 999 & 2.015 & 0.058 & 0.062 & \\
\hline Clindamycin & Clindamycin & 17 & 999 & 5.082 & 0.001 & 0.001 & ** \\
\hline Diet control (study 4) & Diet control (study 4) & 20 & 999 & 2.140 & 0.028 & 0.031 & * \\
\hline
\end{tabular}

The q-value was described above but is calculated differently. Diet control animals of study 1,2 and 4 and the vehicle controls (CMC and corn oil) showed a minor, yet significant difference $(\mathrm{F}=2.1$ - 4.9, $\mathrm{p}<0.05)$ between male and female animals, as well as roxithromycin and clindamycin $(\mathrm{F}=3.5-5.1, \mathrm{p}<0.05)$. However, when comparing $\mathrm{F}$ values here to $\mathrm{F}$ values of treated groups versus control groups where $\mathrm{F}$ is about 50 , the 
differences, as shown in the PCoA, were marginal. Furthermore, as discussed above, feces and cecum content from male and female animals showed a very similar metabolite profile. This led to the assumption that there were no significant differences between sexes regarding the gut microbial communities which could be demonstrated with this evaluation.

Further, genetic factors and interindividual differences of the animals as well as the DNA extraction method could lead to a variability of control animals between independently conducted studies. To elucidate the variability between the control groups of male and female animals of the four studies, the same analyses of alpha and beta diversity were performed. Since the conducted studies were highly standardized regarding age, diet, handling and treatment of the animals, we assumed a low variability between the controls. As seen in Table 3, the controls showed a minor, yet significant, difference in alpha diversity between study control 1,2 and 3 versus study control 4 of female animals, and between study control 1 and 4 versus study control 3 of male animals.

The PERMANOVA analysis in Table 4 for the beta diversity of weighted UniFrac distance revealed that almost all diet control groups showed minor, yet significant, differences when compared to each other both in the male animals $(\mathrm{F}=1.8-3.3, \mathrm{p}<0.05)$ and the female animals $(\mathrm{F}=2.2-5.3, \mathrm{p}<0.05)$. However, as observed for the comparison of male versus female community, the $\mathrm{F}$ value was very low. That means the differences between the control groups, as also shown in the PCoA, were minor. Since the highest variability arises from the 16S rRNA taxonomic profiling due to the DNA extraction step [50], the variability from the genetic background and potential interindividual differences of the Wistar rats in our studies can be considered to be negligible.

Table 3 Differences in alpha diversity of the diet controls from study 1, 2,3 and 4 of female and male animals. Effective species number (Chao et al., 2010) was calculated for each sample by taking the exponential of Shannon-Weiner index and Kruskal-Wallis statistics were calculated using the diversity plugin of QIIME2. The last column indicates where the p value is $<0.05\left(^{*}\right)$ or $<0.01(* *)$.

\begin{tabular}{|c|c|c|c|c|c|c|}
\hline & Group 1 & Group 2 & $\mathbf{H}$ & p-value & q-value & Significance \\
\hline \multirow{6}{*}{ 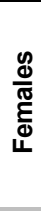 } & Diet control (study $1, n=10$ ) & Diet control (study $2, \mathrm{n}=8$ ) & 0.284 & 0.594 & 0.650 & \\
\hline & " & Diet control (study $3, n=9$ ) & 0.667 & 0.414 & 0.473 & \\
\hline & $"$ & Diet control (study $4, n=10$ ) & 10.080 & 0.001 & 0.003 & ** \\
\hline & Diet control (study $2, \mathrm{n}=8$ ) & Diet control (study $3, n=9$ ) & 0.083 & 0.773 & 0.805 & \\
\hline & " & Diet control (study $4, n=10$ ) & 6.189 & 0.013 & 0.020 & * \\
\hline & Diet control (study $3, n=9$ ) & Diet control (study $4, n=10$ ) & 4.167 & 0.041 & 0.058 & * \\
\hline \multirow{6}{*}{$\frac{\mathscr{d}}{\frac{\pi}{\Sigma}}$} & Diet control (study $1, n=10$ ) & Diet control (study $2, n=10$ ) & 0.206 & 0.650 & 0.702 & \\
\hline & " & Diet control (study $3, n=10$ ) & 0.006 & 0.940 & 0.940 & ** \\
\hline & " & Diet control (study $4, n=10$ ) & 1.286 & 0.257 & 0.313 & \\
\hline & Diet control (study $2, n=10$ ) & Diet control (study $3, n=10$ ) & 0.280 & 0.597 & 0.650 & \\
\hline & " & Diet control (study $4, n=10$ ) & 0.463 & 0.496 & 0.555 & \\
\hline & Diet control (study $3, n=10$ ) & Diet control (study $4, n=10$ ) & 0.015 & 0.903 & 0.912 & * \\
\hline
\end{tabular}


Table 4 Pairwise PERMANOVA results from QIIME2 beta group significance. Diet control animals of study 1, 2, 3 and 4 show a minor, yet significant, difference between study 1 and 4, 2 and 4, and 3 and 4 of female animals, as well as study 1 and 3, and 3 and 4 of male animals. The last column indicates where the p value is $<$ $0.05(*)$ or $<0.01(* *)$.

\begin{tabular}{|c|c|c|c|c|c|c|c|c|}
\hline & Group1 & Group2 & Sample Size & Permutations & pseudo-F & p-value & q-value & Significance \\
\hline \multirow{6}{*}{ 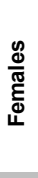 } & Diet control (study 1) & Diet control (study 2) & 18 & 999 & 3.871 & 0.002 & 0.003 & $* *$ \\
\hline & " & Diet control (study 3) & 19 & 999 & 2.213 & 0.003 & 0.004 & $* *$ \\
\hline & $"$ & Diet control (study 4) & 20 & 999 & 5.337 & 0.001 & 0.001 & ** \\
\hline & Diet control (study 2) & Diet control (study 3) & 17 & 999 & 1.556 & 0.122 & 0.127 & \\
\hline & " & Diet control (study 4) & 18 & 999 & 2.918 & 0.014 & 0.016 & * \\
\hline & Diet control (study 3) & Diet control (study 4) & 19 & 999 & 3.867 & 0.004 & 0.005 & ** \\
\hline \multirow{6}{*}{$\frac{\boldsymbol{d}}{\frac{\mathbb{d}}{\mathbb{N}}}$} & Diet control (study 1) & Diet control (study 2) & 20 & 999 & 1.170 & 0.296 & 0.297 & \\
\hline & " & Diet control (study 3) & 20 & 999 & 1.789 & 0.045 & 0.049 & * \\
\hline & " & Diet control (study 4) & 20 & 999 & 2.581 & 0.014 & 0.016 & * \\
\hline & Diet control (study 2) & Diet control (study 3) & 20 & 999 & 1.823 & 0.026 & 0.029 & * \\
\hline & " & Diet control (study 4) & 20 & 999 & 3.301 & 0.001 & 0.001 & ** \\
\hline & Diet control (study 3) & Diet control (study 4) & 20 & 999 & 1.953 & 0.026 & 0.029 & * \\
\hline
\end{tabular}

The data obtained from the four studies with diet controls, vehicle controls and antibiotics having different activity spectra allows a broad correlation analysis and possibly an integration of these data into new systems biology models linking species to specific metabolites. Therefore, a deeper exploration into more specific taxonomic groups (e.g. genera or species) and their metabolic functionality has to be performed in the future. Yet, it has to be noted that the datasets and metagenomic information of the rodent gut need further improvement and characterization: while over 1500 species have been isolated and cultured from the human gut, e.g. only around 100 species have been cultured from mice strains [6,59-61]. Since the feces turned out to be a more sensitive matrix than plasma regarding changes in the metabolome, the correlation analyses should be based upon these data and then linked to the results of the plasma metabolomics. This could allow the estimation of the extent of changes induced in the microbiome with respect to consequences for the host and could enable the identification of more relevant key metabolites derived from the gut microbiome. 


\section{FUTURE PERSPECTIVES}

The gut microbiome uses its genome to fulfil important functions for its host such as the extraction of calories from otherwise indigestible components of the diet, synthesis of essential vitamins and amino acids, bile acid and lipid metabolism, support of the development of the immune system, maintenance of the energy balance, prevention of infections, and many others [5,62-69]. As diverse as these functions of the gut microbiome in the body are, as diverse is the field of research. Hereafter, the following facets of gut microbial research directions will be briefly addressed: I) the gut-brain axis, II) the gut and immune system, III) the gut in health and disease, IV) effects of xenobiotics on the gut microbiome, V) effects of the gut microbiome on xenobiotics including effects on their metabolism and systemic bioavailability, VI) availability and suitability of in vitro models, and VII) including the gut microbiota in ADME and PBK models. The relevance of the gut microbiome in these fields will be set in context with toxicology, pharmacology and the use of metabolomics, and finally the section closes with VIII) a conclusion by summarizing the main findings of this thesis and how it contributed to this field of research.

\section{I) The gut-brain axis}

Between the gut microbiome and the brain exists a bi-directional crosstalk through the gutbrain axis which can influence for example behavior, serotonin metabolism, intestinal gluconeogenesis, the blood-brain barrier, or appetite regulation [70]. The gut itself is embedded in the enteric nervous system (ENS) and can communicate with the central nervous system (CNS), for example via the vagus nerve to the brain. Various diseases, e.g. Alzheimer's disease, autism spectrum disorder, and depression are associated with alterations in gut microbial composition, diversity and the metabolites derived from the gut microbiota. For example, increased levels of short chain fatty acids as well as ammonia were observed in children with autism spectrum disorder, however, a causative role of the gut microbiome remains to be determined [71]. In general, microbiome-derived or associated metabolites are constantly sensed by immune cells and enteric glial cells which can lead to a secretion of neuro-modulatory signaling molecules affecting the CNS [72]. There are various experimental models for these neurological diseases, such as chronic antibiotics treatment and germ-free animal models, fecal microbiome transplantations from human patients or animal models into antibiotic-treated- or germ-free animals, co-housing of animal models with naive or specifically colonized controls, treatment with prebiotics (constituents in food that induce the growth or activity of beneficial microbes), probiotics (live microorganisms intended to provide health benefits), synbiotics (combination of preand probiotics), or postbiotics (byproducts of probiotic metabolism) to colonize the gut with selected microbiome of choice, and application of culturomics methodologies to isolate potential disease-relevant bacterial strains [72]. Elucidating the mechanisms behind the complex crosstalk could help to discover for example new drug candidates for patients suffering from neurodegenerative diseases such as Parkinson's and Alzheimer's disease or multiple sclerosis, which are of major public health concern. Pro-, pre-, post- and synbiotics 
could be promising health ingredients able to modulate gut microbiota composition and thereby delaying or preventing the onset or progression of these diseases. There is a need to systematically identify the pathogenic mechanisms, to characterize gut community and functional changes and, on this basis, develop a concept for a targeted, e.g. nutritional, intervention.

\section{II) The gut and immune system}

The gut microbiome plays a crucial role in the development and homeostasis of the host mammalian immune system, especially during infancy. The microbes communicate with the host cells, locally and systemically, via metabolites. For instance, short-chain fatty acids have broad effects on the immune system and can directly or indirectly act on local or resident antigen-presenting cells to decrease inflammatory responses associated with neuroinflammation and allergic airway disease [73]. As research demonstrated, there are several microbes having well-characterized effects on particular immune functions [74,75]. The current mechanistic understanding reveals a complex interplay since microbial interactions with immunity are affected by co-variables as location, timing, and context [76]. Caesarean sections and a critical period during early infancy regarding the development of the immune system are associated with persistent defects in the development and training of specific immune subsets due to the disruption of microbiomehost interactions, which can lead to the development of diseases later on, e.g. diabetes, allergy, asthma or inflammatory bowel disease [42,77]. As already stated above, antibiotic use, especially during this "window of opportunity", can have short-term and long-term consequences on the human microbiome and thus on the host immune system [42]. Therefore, elucidating the mechanisms of the host-microbe interactions, especially in this vulnerable period of time, is essential to prevent these diseases, to develop alternative therapeutic approaches and to critically reconsider the often excessive and imprudent use of antibiotics.

\section{III) The gut in health and disease}

A shift or perturbations of the microbiome, especially during the first years of life, have been associated with diseases such as Crohn's disease, colon cancer, diabetes, allergies, Alzheimer's and Parkinson's disease, depression, stroke, and many more [2]. As described above, microbes and their metabolites have the potential to affect the host organism in a detrimental or beneficial way. The findings of associations between these microbes/their metabolites and diseases, or prevention from disease, can be used to develop new therapeutic strategies for diverse purposes. For example, fecal microbiota transplants from a healthy donor have outstanding efficacy in treating Clostridium difficile infections [78]. Regarding obesity, studies in germ-free mice receiving fecal microbiota transplants from conventionally raised mice have provided the most compelling evidence establishing the causal link between gut microbes and energy harvest from the diet and energy storage in the host [79]. Another aspect is the importance of the gut microbiome in the treatment of 
cancer. It is hypothesized that there exists a cross-reactivity between microbial and tumor antigens shaping $\mathrm{T}$ cell repertoires and/or microbial products stimulating pattern recognition receptors that influence the type and intensity of immune responses [80]. As studies in patients demonstrated, the gut microbiome influenced the efficacy of immune checkpoint inhibitors against epithelial tumors: superior efficacy is observed with the presence of specific gut microbes, however, it can be diminished with administration of antibiotics $[81,82]$. Considering these examples, a better understanding of the functionality of the microbiome will be useful for risk assessment, novel diagnostics and therapeutic approaches, e.g. with pre-, pro-, syn- or probiotics, and for the establishment of future strategies of personalized or precision medicine.

\section{IV) Effects of xenobiotics on the gut microbiome}

There is evidence that exposure to xenobiotics, both chemicals and drugs, can affect the gut microbial composition and that this is associated with neurobehavioral, immunological, metabolic, gastrointestinal, cardiovascular diseases and even cancer, although not for all causality has been established. Endocrine disrupting chemicals, heavy metals, air pollution, and nanoparticles can affect the host-microbial interaction by inducing a shift of the gut microbial community or altering its functionality [4]. Further, pesticides may play a role for living organisms in our ecosystem [83]. Also the consumption of drugs, such as the antidiabetic metformin [84], nonsteroidal anti-inflammatory drugs [85], or antibiotics as shown in this thesis, has been associated with changes in the gut microbial composition. In a recent study, more than 1,000 marketed drugs from different therapeutic classes were screened against 40 representative gut bacterial strains, and found that $24 \%$ of the drugs inhibited the growth of at least one strain in vitro [86]. It would thus seem that nearly everything has an effect on the gut microbiome. However, it is often not clear if such changes of the gut microbiome are associated with any form of adverseness and increased efforts to establish causality are needed which also applies to research communication. The potential risk of xenobiotics and drugs needs further exploration, and suitable methods have to be developed to analyze gut microbiome changes which might subsequently induce pathophysiological responses in the host. As microbiome-derived or -associated metabolites seem to play a central role in these pathogenic mechanisms, the use of metabolomics could help to develop prevention and remediation strategies.

\section{V) Effects of the gut microbiome on xenobiotics including effects on their metabolism and systemic bioavailability}

The intestinal microbes possess a metabolic capacity to biotransform xenobiotics. This is of direct relevance for pharmacology and toxicology since this can directly and indirectly affect the xenobiotic metabolism of the host. The processes the microbes mediate are poorly understood and experimental systems to address this complex process of gut microbial metabolic capacity are lacking. Microbial transformation mechanisms include processes like reduction, hydrolysis, removal of succinate groups, dehydroxylation, de- 
/acetylation, cleavage of N-oxide bonds, proteolysis, or denitration [87], which can lead to the formation of metabolites with increased or decreased toxicity [88]. Indirect interactions have also been described, including microbial effects on the host gene expression, and activity of components of host xenobiotic metabolism, which finally leads to an altered host (drug) metabolism [89]. The consequence of the microbial metabolism was demonstrated in many cases, e.g. in Japan in the late 1990s, 18 patients died caused by interactions of sorivudine, an antiviral drug, with the cancer drug 5-fluorouracil, when the metabolite bromovinyluracil produced by the gut microbiota inhibited an enzyme dihydropyrimidine dehydrogenase (DPD) in the host responsible for detoxification of the cancer drug [88]. In general, there is a lack of knowledge in understanding the potential microbial contribution to substance toxification and exposure. In this context, it is of interest to note that the gut microbiome is not (yet) included in the ADME - absorption, distribution, metabolism, excretion - concept used in the pharmacological and toxicological field, e.g. acting as first pass metabolism thereby influencing the nature and amount of the actual absorbed dose [90]. However, this knowledge and data might be essential for paving the way for a new era of toxicology where in vivo experiments shall be more and more replaced by alternative methods and modeling in which the effects of the gut microbiota are no longer implicitly included in the safety testing.

\section{VI) Availability and suitability of in vitro models}

In mechanistic and experimental studies, rodents have frequently been used as animal models exploring the correlations between gastrointestinal microorganisms and diseases and have significantly contributed to the understanding of the microbiome. However, as mentioned before there are significant interindividual and interspecies differences in the composition of the microbiome. As described in Chapter 1, analyses of the rat intestinal microbiota revealed a bacterial diversity exceeding that of the human gut by a factor of two to three [91]. The phylogenetic classification suggested that the rodent (rats and mice) and human microbiome are similar at the phylum level, but different at the genus level [14]. There is a lack of knowledge in understanding the potential microbial contribution to substance (de)toxification and to which extent these results are transferable between rodents, in vitro models and humans. Interestingly, contrary to differences in the human and murine intestinal genera, almost $80 \%$ of the functional genes of the murine microbiome were identical to the human microbiome, indicating a functional overlap [92]. Similarly, in the human microbiome, the interindividual variation in expression of bacterial metabolic enzymes is less diverse than the taxonomic profiles [93]. That means that determining the functional endpoints rather than the composition can contribute to a greater understanding of how microbial communities function, their complex interactions and metabolic capacities, also in close interaction with the host. Thus, to develop appropriate in vitro models an essential first step is to measure the metabolite profile in different matrices, e.g. feces, from rodents and humans, in order to identify species-specific effects and to possibly reveal individual susceptibility to chemical toxicity or pharmacological effects. 
Metagenomic tools could be applied to mine the genome for metabolic enzymes and interspecies differences. Analyzing different endpoints, as the metabolome in different matrices as plasma and feces, compositional changes in the microbiome after treatment with a substance, and the modified xenobiotics itself to capture inter- and intraspecies differences could be the first stage of a series of investigations that have the ultimate goal of assessing the pharmacological benefit or toxicological risk of new compounds regarding the gut microbiome.

\section{VII) Including the gut microbiota in ADME and PBK models}

Even though humans and rodents have many similarities in their intestine regarding physiological, anatomical and histological features, there are not only differences in size, metabolic rate and dietary habits but also profound differences in their microbial composition [6]. This raises the question to which extent the results of animal models are transferable between rodents and humans. However, so far there is no better alternative to the animal model to study the mechanistic and metabolic capacity of the gut microbiome and its role in health and disease. Regarding the (xenobiotic) metabolic capacity of the gut microbiome and the large intra- and interindividual variability, as a first step it is essential to understand how xenobiotic compounds are biotransformed by resident microbiota and how this influences the composition and functionality of the microbiome. Therefore, novel tools and new methods have to be developed to give detailed mechanistic understanding of the gut microbiome. In consideration of the Three Rs - replacement, refinement, reduction - concept there is a need of appropriate in vitro models which could gain insight into interspecies and interindividual differences with the final goal to extrapolate from in vitro to in vivo data. In vivo models in rodents should be compared to anaerobic in vitro models using rat and human feces, and a representative standard community. With these models interspecies and interindividual differences in the intestinal microbial metabolism of the same xenobiotic, e.g. (foodborne) chemicals or pharmaceuticals, of pharmacological or toxicological relevance could be elucidated. The transformation of in vitro to in vivo data requires profound knowledge about the differences between the functionality and metabolic capacity of the gut microbiome. Therefore, an approach to integrate intestinal microbioal metabolic capacity in an in vitro to in vivo extrapolation (IVIVE) has to be developed. In vitro data have to be extrapolated to in vivo effects, as well as animal to human responses. These results can be correlated with in vitro models to pave the way for future humanrelevant in vitro based risk assessment strategies, that include interspecies and interindividual differences in the IVIVE and physiologically-based kinetic (PBK) modeling used. Here, metabolic rates can be defined and incorporated into the models. Concentrationresponse curves for chemical-induced changes in key metabolites can be used to define relevant parameters for PBK modeling based reverse dosimetry [94]. In general, mathematical modeling-based methods have the potential to generate new insights of the gut microbiome in health and disease $[95,96]$. For example, a systems biology approach utilizing the metabolome and taxonomic profiling data can be used to correlate these results 
to identify species of the rodent and human microbiome that contribute to its metabolic output and share functions. In a study of Stein et al., they developed and demonstrated in a first-of-a-kind experimental validation the usefulness of a mathematical microbiomeimmune system model which the authors called a stepping-stone to the accelerated prototyping and rational design of microbiome therapies [97].

\section{VIII) Conclusion}

Regarding the complexity of the microbiome itself and the tight host-microbe interactions, there are still many challenges to overcome. Since in both pharmacological and toxicological research the examined and evaluated endpoints are in the majority of cases systemic effects, one may query the role of the gut microbiome for these endpoints. Nevertheless, the fundamental role of the intestinal microbiome for the health of a host organism is nowadays generally accepted. It is known since decades that the intestinal microbiome has an influence on the metabolism of food-born constituents and xenobiotics. Hence, it is of utmost importance for future research directions in pharmacology and (eco)toxicology not just to understand the gut microbiome's role in the absorption of xenobiotics, but also to understand whether gut microbial changes have an impact on the metabolic functionality of the microbiome and in how far this can affect the intestinal metabolism as well as the systemic bioavailability of model compounds including both drugs and other xenobiotics, as well as endogenous metabolites. As emerges from the gut microbial research directions described above as well as in this thesis, a key aspect is despite this wide variety of research fields - the gut microbial functionality. With this thesis, the first stage of investigations assessing the functionality of the microbiome using metabolomics in the field of microbiome and toxicology were performed. The results suggest that plasma based metabolic profiling is a suitable tool to investigate the functionality of the gut microbiome, and laid the basis for the development of a promising method to elucidate the underlying mechanisms leading to adverse effects in the host system. 


\section{REFERENCES}

1. Modi SR, Collins JJ, Relman D a. Antibiotics and the gut microbiota. J Clin Invest. 2014;124: 4212-4218. doi:10.1172/JCI72333.themselves

2. Zhang Y-J, Li S, Gan R-Y, Zhou T, Xu D-P, Li H-B. Impacts of Gut Bacteria on Human Health and Diseases. Int J Mol Sci. 2015;16: 7493-7519. doi:10.3390/ijms16047493

3. David L a, Maurice CF, Carmody RN, Gootenberg DB, Button JE, Wolfe BE, et al. Diet rapidly and reproducibly alters the human gut microbiome. Nature. Nature Publishing Group; 2014;505: 559-63. doi:10.1038/nature12820

4. Rosenfeld CS. Gut Dysbiosis in Animals Due to Environmental Chemical Exposures. Front Cell Infect Microbiol. 2017;7. doi:10.3389/fcimb.2017.00396

5. Bäumler AJ, Sperandio V. Interactions between the microbiota and pathogenic bacteria in the gut. Nature. 2016;535: 85-93. doi:10.1038/nature18849

6. Hugenholtz F, de Vos WM. Mouse models for human intestinal microbiota research: a critical evaluation. Cell Mol Life Sci. Springer International Publishing; 2018;75: 149-160. doi:10.1007/s00018-017-2693-8

7. McCoy KD, Geuking MB, Ronchi F. Gut microbiome standardization in control and experimental mice. Curr Protoc Immunol. 2017;2017: 23.1.1-23.1.13. doi:10.1002/cpim. 25

8. Cho I, Blaser MJ. The human microbiome: at the interface of health and disease. Nat Rev Genet. 2012;13: 260-70. doi:10.1038/nrg3182

9. Adir J. Enterohepatic circulation of tetracycline in rats. J Pharm Sci. Elsevier Masson SAS; 1975;64: 1847-50. doi:10.1002/jps.2600641121

10. Musther H, Olivares-Morales A, Hatley OJD, Liu B, Rostami Hodjegan A. Animal versus human oral drug bioavailability: Do they correlate? Eur J Pharm Sci. Elsevier B.V.; 2014;57: 280-291. doi:10.1016/j.ejps.2013.08.018

11. Vargas-Estrada D, Gutiérrez L, Juarez-Rodríguez I, Sumano H. Pharmacokinetics of doxycycline and tissue concentrations of an experimental long-acting parenteral formulation of doxycycline in Wistar rats. Arzneimittelforschung. 2008;58: 310315. doi:10.1055/s-0031-1296512.Pharmacokinetics

12. Brown SA, Riviere JE. Comparative pharmacokinetics of amionoglycoside antibiotics. J Vet Pharmacol Ther. 1991;14: 1-35. doi:10.3109/14992027.2010.524253

13. Renshaw D, Cerniglia C, Mitsumori K. NEOMYCIN (addendum, WHO Food Additives Series 51) [Internet]. [cited 11 Aug 2016]. Available: http://www.inchem.org/documents/jecfa/jecmono/v51je02.htm

14. Li D, Chen H, Mao B, Yang Q, Zhao J, Gu Z, et al. Microbial Biogeography and Core Microbiota of the Rat Digestive Tract. Sci Rep. Nature Publishing Group; 2017;8:45840. doi:10.1038/srep45840

15. Lindon JC, Holmes E, Bollard ME, Stanley EG, Nicholson JK. Metabonomics technologies and their applications in physiological monitoring, drug safety assessment and disease diagnosis. Biomarkers. 2004;9: 1-31. 
doi:10.1080/13547500410001668379

16. van Ravenzwaay B, Herold M, Kamp H, Kapp MD, Fabian E, Looser R, et al. Metabolomics: A tool for early detection of toxicological effects and an opportunity for biology based grouping of chemicals-From QSAR to QBAR. Mutat Res - Genet Toxicol Environ Mutagen. Elsevier B.V.; 2012;746: 144-150. doi:10.1016/j.mrgentox.2012.01.006

17. Beger RD, Sun J, Schnackenberg LK. Metabolomics approaches for discovering biomarkers of drug-induced hepatotoxicity and nephrotoxicity. Toxicology and applied pharmacology. 2010. pp. 154-166. doi:10.1016/j.taap.2009.11.019

18. van Ravenzwaay B, Sperber S, Lemke O, Fabian E, Faulhammer F, Kamp H, et al. Metabolomics as read-across tool: A case study with phenoxy herbicides. Regul Toxicol Pharmacol. Elsevier Ltd; 2016;81: 288-304. doi:10.1016/j.yrtph.2016.09.013

19. Honneffer JB, Steiner JM, Lidbury J a., Suchodolski JS. Variation of the microbiota and metabolome along the canine gastrointestinal tract. Metabolomics. Springer US; 2017;13: 26. doi:10.1007/s11306-017-1165-3

20. Sun J, Schnackenberg LK, Khare S, Yang X, Greenhaw J, Salminen W, et al. Evaluating effects of penicillin treatment on the metabolome of rats. J Chromatogr B Anal Technol Biomed Life Sci. Elsevier B.V.; 2013;932: 134-143. doi:10.1016/j.jchromb.2013.05.030

21. Swann JR, Tuohy KM, Lindfors P, Brown DT, Gibson GR, Wilson ID, et al. Variation in antibiotic-induced microbial recolonization impacts on the host metabolic phenotypes of rats. J Proteome Res. 2011;10: 3590-3603. doi: $10.1021 / \mathrm{pr} 200243 \mathrm{t}$

22. Zheng X, Xie G, Zhao A, Zhao L, Yao C, Chiu NHL, et al. The footprints of gut microbial-mammalian co-metabolism. J Proteome Res. 2011;10: 5512-5522. doi:10.1021/pr2007945

23. Anderson TJ, Jones RW, Ai Y, Houk RS, Jane J, Zhao Y, et al. High-resolution time-of-flight mass spectrometry fingerprinting of metabolites from cecum and distal colon contents of rats fed resistant starch. Anal Bioanal Chem. 2014;406: 745-756. doi:10.1007/s00216-013-7523-8

24. Williams RE, Eyton-Jones HW, Farnworth MJ, Gallagher R, Provan WM. Effect of intestinal microflora on the urinary metabolic profile of rats: a 1 H-nuclear magnetic resonance spectroscopy study. Xenobiotica. 2002;32: 783-794. doi:10.1080/00498250210143047

25. Nicholls AW, Mortishire-Smith RJ, Nicholson JK. NMR Spectroscopic-Based Metabonomic Studies of Urinary Metabolite Variation in Acclimatizing Germ-Free Rats. Chem Res Toxicol. 2003;16: 1395-1404. doi:10.1021/tx0340293

26. Wikoff WR, Anfora AT, Liu J, Schultz PG, Lesley S a, Peters EC, et al. Metabolomics analysis reveals large effects of gut microflora on mammalian blood metabolites. Proc Natl Acad Sci U S A. 2009;106: 3698-3703. doi:10.1073/pnas.0812874106

27. Li J V., Swann J, Marchesi JR. Biology of the Microbiome 2: Metabolic Role. Gastroenterol Clin North Am. Elsevier Inc; 2017;46: 37-47. 
doi:10.1016/j.gtc.2016.09.006

28. Sharma V, Smolin J, Nayak J, Ayala JE, Scott DA, Peterson SN, et al. Mannose Alters Gut Microbiome, Prevents Diet-Induced Obesity, and Improves Host Metabolism. Cell Rep. ElsevierCompany.; 2018;24: 3087-3098. doi:10.1016/j.celrep.2018.08.064

29. Behr C, Kamp H, Fabian E, Krennrich G, Mellert W, Peter E, et al. Gut microbiome-related metabolic changes in plasma of antibiotic-treated rats. Arch Toxicol. Springer Berlin Heidelberg; 2017;91: 3439-3454. doi:10.1007/s00204017-1949-2

30. Ramezani A, Massy Z a., Meijers B, Evenepoel P, Vanholder R, Raj DS. Role of the Gut Microbiome in Uremia: A Potential Therapeutic Target. Am J Kidney Dis. 2015; 1-16. doi:10.1053/j.ajkd.2015.09.027

31. Strahl N, Barr W. Intestinal drug absorption and metabolism. 3. Glycine conjugation and accumulation of benzoic acid in rat intestinal tissue. J Pharm Sci. 1971;60: 278-81.

32. Chiba M, Poon K, Hollands J, Pang K. Glycine conjugation activity of benzoic acid and its acinar localization in the perfused rat liver. J Pharmacol Exp Ther. 1994;268: 409-16.

33. Poon K, Pang K. Benzoic acid glycine conjugation in the isolated perfused rat kidney. Drug Metab Dispos. 1995;23: 255-60.

34. Nicholson JK, Holmes E, Kinross J, Burcelin R, Gibson G, Jia W, et al. Host-Gut Microbiota Metabolic Interactions. 2012;108: 1262-1268.

35. Lechner SM. Glutamate-based therapeutic approaches: Inhibitors of glycine transport. Current Opinion in Pharmacology. 2006. pp. 75-81. doi:10.1016/j.coph.2005.11.002

36. García-Cañaveras JC, Donato MT, Castell J V., Lahoz A. Targeted profiling of circulating and hepatic bile acids in human, mouse, and rat using a UPLC-MRMMS-validated method. J Lipid Res. 2012;53: 2231-2241. doi:10.1194/jlr.D028803

37. Zierer J, Jackson MA, Kastenmüller G, Mangino M, Long T, Telenti A, et al. The fecal metabolome as a functional readout of the gut microbiome. Nat Genet. Springer US; 2018;50: 790-795. doi:10.1038/s41588-018-0135-7

38. Strauss V, Wiemer J, Leibold E, Kamp H, Walk T, Mellert W, et al. Influence of strain and sex on the metabolic profile of rats in repeated dose toxicological studies. Toxicol Lett. 2009;191: 88-95. doi:10.1016/j.toxlet.2009.08.004

39. Marchant J. When antibiotics turn toxic. Nature. 2018; doi:10.1038/d41586-01803267-5

40. Gao Z, Chen Y, Guan M-X. Mitochondrial DNA Mutations Associated with Aminoglycoside Ototoxicity. J Otol. 2006;1: 65-75. doi:10.1016/S16722930(06)50016-9

41. Badal S, Her YF, James Maher L. Nonantibiotic effects of fluoroquinolones in mammalian cells. J Biol Chem. 2015;290: 22287-22297. doi:10.1074/jbc.M115.671222 
42. Langdon A, Crook N, Dantas G. The effects of antibiotics on the microbiome throughout development and alternative approaches for therapeutic modulation. Genome Med. Genome Medicine; 2016;8: 39. doi:10.1186/s13073-016-0294-z

43. Lane DJ, Pace B, Olsen GJ, Stahl DA, Sogin ML. Rapid determination of 16S ribosomal RNA sequences for phylogenetic analyses. Proc Natl Acad Sci USA. 1986;583: 1453-1457. Available: http://www.pnas.org/content/pnas/82/20/6955.full.pdf

44. Gloor GB, Wu JR, Pawlowsky-Glahn V, Egozcue JJ. It's all relative: analyzing microbiome data as compositions. Ann Epidemiol. Elsevier Inc; 2016;26: 322-329. doi:10.1016/j.annepidem.2016.03.003

45. Vandeputte D, Kathagen G, D’Hoe K, Vieira-Silva S, Valles-Colomer M, Sabino J, et al. Quantitative microbiome profiling links gut community variation to microbial load. Nature. Nature Publishing Group; 2017;551: 507-511. doi:10.1038/nature24460

46. Bender JM, Li F, Adisetiyo H, Lee D, Zabih S, Hung L, et al. Quantification of variation and the impact of biomass in targeted $16 \mathrm{~S}$ rRNA gene sequencing studies. Microbiome; 2018; 1-12. doi:10.1186/s40168-018-0543-Z

47. Louca S, Doebeli M, Parfrey LW. Correcting for 16S rRNA gene copy numbers in microbiome surveys remains an unsolved problem. Microbiome. Microbiome; 2018;6: 41. doi:10.1186/s40168-018-0420-9

48. Gasc C, Peyret P. Hybridization capture reveals microbial diversity missed using current profiling methods. Microbiome. Microbiome; 2018;6: 61. doi:10.1186/s40168-018-0442-3

49. Kleiner M, Thorson E, Sharp CE, Dong X, Liu D, Li C, et al. Assessing species biomass contributions in microbial communities via metaproteomics. Nat Commun. Springer US; 2017;8. doi:10.1038/s41467-017-01544-X

50. Costea PI, Zeller G, Sunagawa S, Pelletier E, Alberti A, Levenez F, et al. Towards standards for human fecal sample processing in metagenomic studies. Nat Biotechnol. Nature Publishing Group; 2017;35: 1069-1076. doi:10.1038/nbt.3960

51. Gerasimidis K, Bertz M, Quince C, Brunner K, Bruce A, Combet E, et al. The effect of DNA extraction methodology on gut microbiota research applications. BMC Res Notes. 2016;9. doi:10.1186/s13104-016-2171-7

52. Kennedy NA, Walker AW, Berry SH, Duncan SH, Farquarson FM, Louis P, et al. The impact of different DNA extraction kits and laboratories upon the assessment of human gut microbiota composition by $16 \mathrm{~S}$ rRNA gene sequencing. PLoS One. 2014;9. doi:10.1371/journal.pone.0088982

53. Choo JM, Leong LEX, Rogers GB. Sample storage conditions significantly influence faecal microbiome profiles. Sci Rep. 2015;5. doi:10.1038/srep16350

54. Knight R, Vrbanac A, Taylor BC, Aksenov A, Callewaert C, Debelius J, et al. Best practices for analysing microbiomes. Nat Rev Microbiol. Springer US; 2018;16: 410-422. doi:10.1038/s41579-018-0029-9

55. Markle JGM, Frank DN, Mortin-toth S, Robertson CE, Feazel LM, Rollekampczyk U, et al. Sex Differences in the Gut Microbiome Drive Hormone- 
Dependent Regulation of Autoimmunity. Science (80- ). 2013;339: 1084-1088. doi:10.1126/science. 1233521

56. Chao A, Chiu C-H, Jost L. Phylogenetic diversity measures based on Hill numbers. Philos Trans R Soc B Biol Sci. 2010;365: 3599-3609. doi:10.1098/rstb.2010.0272

57. Anderson MJ. Permutational Multivariate Analysis of Variance (PERMANOVA). Wiley StatsRef Stat Ref Online. 2017; 1-15. doi:10.1002/9781118445112.stat07841

58. Buttigieg PL, Ramette A. A guide to statistical analysis in microbial ecology: A community-focused, living review of multivariate data analyses. FEMS Microbiol Ecol. 2014;90: 543-550. doi:10.1111/1574-6941.12437

59. Lagier JC, Khelaifia S, Alou MT, Ndongo S, Dione N, Hugon P, et al. Culture of previously uncultured members of the human gut microbiota by culturomics. Nat Microbiol. 2016;1. doi:10.1038/nmicrobiol.2016.203

60. Rajilić-Stojanović M, de Vos WM. The first 1000 cultured species of the human gastrointestinal microbiota. FEMS Microbiol Rev. 2014;38: 996-1047. doi:10.1111/1574-6976.12075

61. Lagkouvardos I, Pukall R, Abt B, Foesel BU, Meier-Kolthoff JP, Kumar N, et al. The Mouse Intestinal Bacterial Collection (miBC) provides host-specific insight into cultured diversity and functional potential of the gut microbiota. Nat Microbiol. 2016;1. doi:10.1038/nmicrobiol.2016.131

62. Wolf G. Gut microbiota: a factor in energy regulation. Nutr Rev. 2006;64: 47-50. doi:10.1301/nr.2006.jan.47

63. Neis EPJG, Dejong CHC, Rensen SS. The Role of Microbial Amino Acid Metabolism in Host Metabolism. Nutrients. 2015;7: 2930-2946. doi:10.3390/nu7042930

64. Forbes JD, Van Domselaar G, Bernstein CN. The gut microbiota in immunemediated inflammatory diseases. Front Microbiol. 2016;7: 1-18. doi:10.3389/fmicb.2016.01081

65. Cani PD, Delzenne NM. The role of the gut microbiota in energy metabolism and metabolic disease. Curr Pharm Des. 2009;15: 1546-1558. doi:10.2174/138161209788168164

66. Sagar NM, Cree IA, Covington JA, Arasaradnam RP. The interplay of the gut microbiome, bile acids, and volatile organic compounds. Gastroenterol Res Pract. 2015;2015: 398585. doi:http://dx.doi.org/10.1155/2015/398585

67. Wilson ID, Nicholson JK. Gut microbiome interactions with drug metabolism, efficacy, and toxicity. Transl Res. Elsevier Inc.; 2017;179: 204-222. doi:10.1016/j.trs1.2016.08.002

68. Lin M, Xie Z, Zhou Y, Li Y, Ren J, Peng X, et al. Dynamic metabonomic and microbiological response of rats to lincomycin exposure: an integrated microbiology and metabonomics analysis. RSC Adv. Royal Society of Chemistry; 2015;5: 65415-65426. doi:10.1039/C5RA10626E

69. Lecomte V, Kaakoush NO, Maloney C a., Raipuria M, Huinao KD, Mitchell HM, et al. Changes in Gut Microbiota in Rats Fed a High Fat Diet Correlate with 
Obesity-Associated Metabolic Parameters. PLoS One. 2015;10: e0126931. doi:10.1371/journal.pone.0126931

70. Schroeder BO, Bäckhed F. Signals from the gut microbiota to distant organs in physiology and disease. Nat Med. 2016;22: 1079-1089. doi:10.1038/nm.4185

71. Wang L, Christophersen CT, Sorich MJ, Gerber JP, Angley MT, Conlon MA. Elevated fecal short chain fatty acid and ammonia concentrations in children with autism spectrum disorder. Dig Dis Sci. 2012;57: 2096-2102. doi:10.1007/s10620012-2167-7

72. Kundu P, Blacher E, Elinav E, Pettersson S. Our Gut Microbiome: The Evolving Inner Self. Cell. Elsevier Inc.; 2017;171: 1481-1493. doi:10.1016/j.cell.2017.11.024

73. Rooks MG, Garrett WS. Gut microbiota, metabolites and host immunity. Nat Rev Immunol. Nature Publishing Group; 2016;16: 341-52. doi:10.1038/nri.2016.42

74. Blaser MJ. The microbiome revolution. J Clin Invest. 2014;124: 4162-4165. doi:10.1172/JCI78366.The

75. Chung H, Pamp SJ, Hill JA, Surana NK, Edelman SM, Troy EB, et al. Gut immune maturation depends on colonization with a host-specific microbiota. Cell. 2012;149: 1578-1593. doi:10.1016/j.cell.2012.04.037

76. Surana NK, Kasper DL. Deciphering the tête-è-tête between the microbiota and the immune system. Journal of Clinical Investigation. 2014. pp. 4197-4203. doi:10.1172/JCI72332

77. Gensollen T, Iyer SS, Kasper DL, Blumberg RS. How colonization by microbiota in early life shapes the immune system. Science (80- ). 2016;352: 539-544. doi:10.1126/science.aad9378

78. Vázquez-Baeza Y, Callewaert C, Debelius J, Hyde E, Marotz C, Morton JT, et al. Impacts of the Human Gut Microbiome on Therapeutics. Annu Rev Pharmacol Toxicol. 2018;58: annurev-pharmtox-042017-031849. doi:10.1146/annurevpharmtox-042017-031849

79. Bäckhed F, Ding H, Wang T, Hooper L V, Koh GY, Nagy A, et al. The gut microbiota as an environmental factor that regulates fat storage. Proc Natl Acad Sci U S A. 2004;101: 15718-23. doi:10.1073/pnas.0407076101

80. Zitvogel L, Ayyoub M, Routy B, Kroemer G. Microbiome and Anticancer Immunosurveillance. Cell. Elsevier Inc.; 2016;165: 276-287. doi:10.1016/j.cell.2016.03.001

81. Routy B, Le Chatelier E, Derosa L, Duong CPM, Alou MT, Daillère R, et al. Gut microbiome influences efficacy of PD-1-based immunotherapy against epithelial tumors. Science (80- ). 2018;359: 91-97. doi:10.1126/science.aan3706

82. Zitvogel L, Ma Y, Raoult D, Kroemer G, Gajewski TF. The microbiome in cancer immunotherapy: Diagnostic tools and therapeutic strategies. Science (New York, N.Y.). 2018. pp. 1366-1370. doi:10.1126/science.aar6918

83. Motta EVS, Raymann K, Moran NA. Glyphosate perturbs the gut microbiota of honey bees. Proc Natl Acad Sci. 2018;2018: 201803880. doi:10.1073/pnas.1803880115 
84. Forslund K, Hildebrand F, Nielsen T, Falony G, Le Chatelier E, Sunagawa S, et al. Disentangling type 2 diabetes and metformin treatment signatures in the human gut microbiota. Nature. 2015;528: 262-266. doi:10.1038/nature15766

85. Rogers MAM, Aronoff DM. The influence of non-steroidal anti-inflammatory drugs on the gut microbiome. Clin Microbiol Infect. 2016;22: 178.e1-178.e9. doi:10.1016/j.cmi.2015.10.003

86. Maier L, Pruteanu M, Kuhn M, Zeller G, Telzerow A, Anderson EE, et al. Extensive impact of non-antibiotic drugs on human gut commensals. Nature. Nature Publishing Group; 2018; doi:10.1038/nature25979.

87. Sousa T, Paterson R, Moore V, Carlsson A, Abrahamsson B, Basit AW. The gastrointestinal microbiota as a site for the biotransformation of drugs. Int J Pharm. 2008;363: 1-25. doi:10.1016/j.ijpharm.2008.07.009

88. Okuda H, Ogura K, Kato A, Takubo H, Watabe T. A possible mechanism of eighteen patient deaths caused by interactions of sorivudine, a new antiviral drug, with oral 5-fluorouracil prodrugs. J Pharmacol Exp Ther. 1998;287: 791-799.

89. Haiser HJ, Turnbaugh PJ. Is it time for a metagenomic basis of therapeutics? Science. 2012;336: 1253-5. doi:10.1126/science.1224396

90. Silbergeld EK. The Microbiome. Toxicol Pathol. 2017;45: 190-194. doi:10.1177/0192623316672073

91. Manichanh C, Reeder J, Gibert P, Varela E, Llopis M, Antolin M, et al. Reshaping the gut microbiome with bacterial transplantation and antibiotic intake. Genome Res. 2010;20: 1411-1419. doi:10.1101/gr.107987.110

92. Krych L, Hansen CHF, Hansen AK, van den Berg FWJ, Nielsen DS. Quantitatively Different, yet Qualitatively Alike: A Meta-Analysis of the Mouse Core Gut Microbiome with a View towards the Human Gut Microbiome. PLoS One. 2013;8. doi:10.1371/journal.pone.0062578

93. Human Microbiome Project Consortium T, Huttenhower C, Gevers D, Knight R, Abubucker S, Badger $\mathrm{JH}$, et al. Structure, function and diversity of the healthy human microbiome. Nature. Nature Publishing Group; 2012;486: 207-214. doi:10.1038/nature11234

94. Louisse J, Beekmann K, Rietjens IMCM. Use of physiologically based kinetic modeling-based reverse dosimetry to predict in vivo toxicity from in vitro data. Chemical Research in Toxicology. 2017. pp. 114-125. doi:10.1021/acs.chemrestox.6b00302

95. Michor F, Beal K. Improving Cancer Treatment via Mathematical Modeling: Surmounting the Challenges Is Worth the Effort. Cell. 2015. pp. 1059-1063. doi:10.1016/j.cell.2015.11.002

96. Rowland I, Gibson G, Heinken A, Scott K, Swann J, Thiele I, et al. Gut microbiota functions: metabolism of nutrients and other food components. Eur J Nutr. Springer Berlin Heidelberg; 2017;0: 1-24. doi:10.1007/s00394-017-1445-8

97. Stein RR, Tanoue T, Szabady RL, Bhattarai SK, Olle B, Norman JM, et al. Computer-guided design of optimal microbial consortia for immune system modulation. Elife. 2018;7: 1-17. doi:10.7554/eLife.30916 



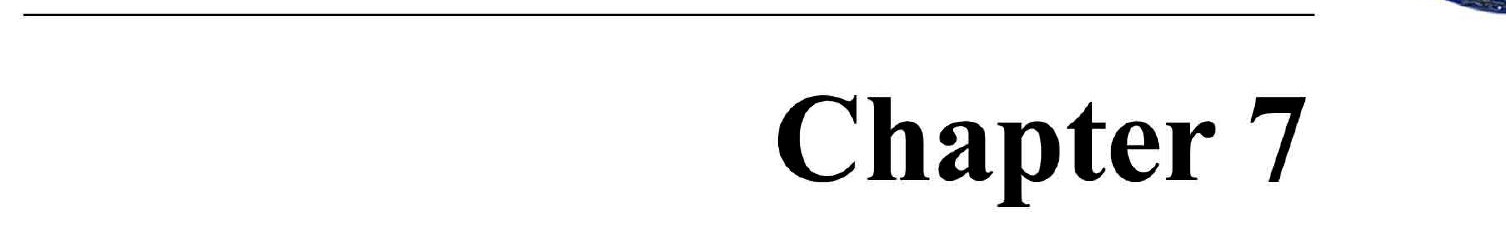

Summary

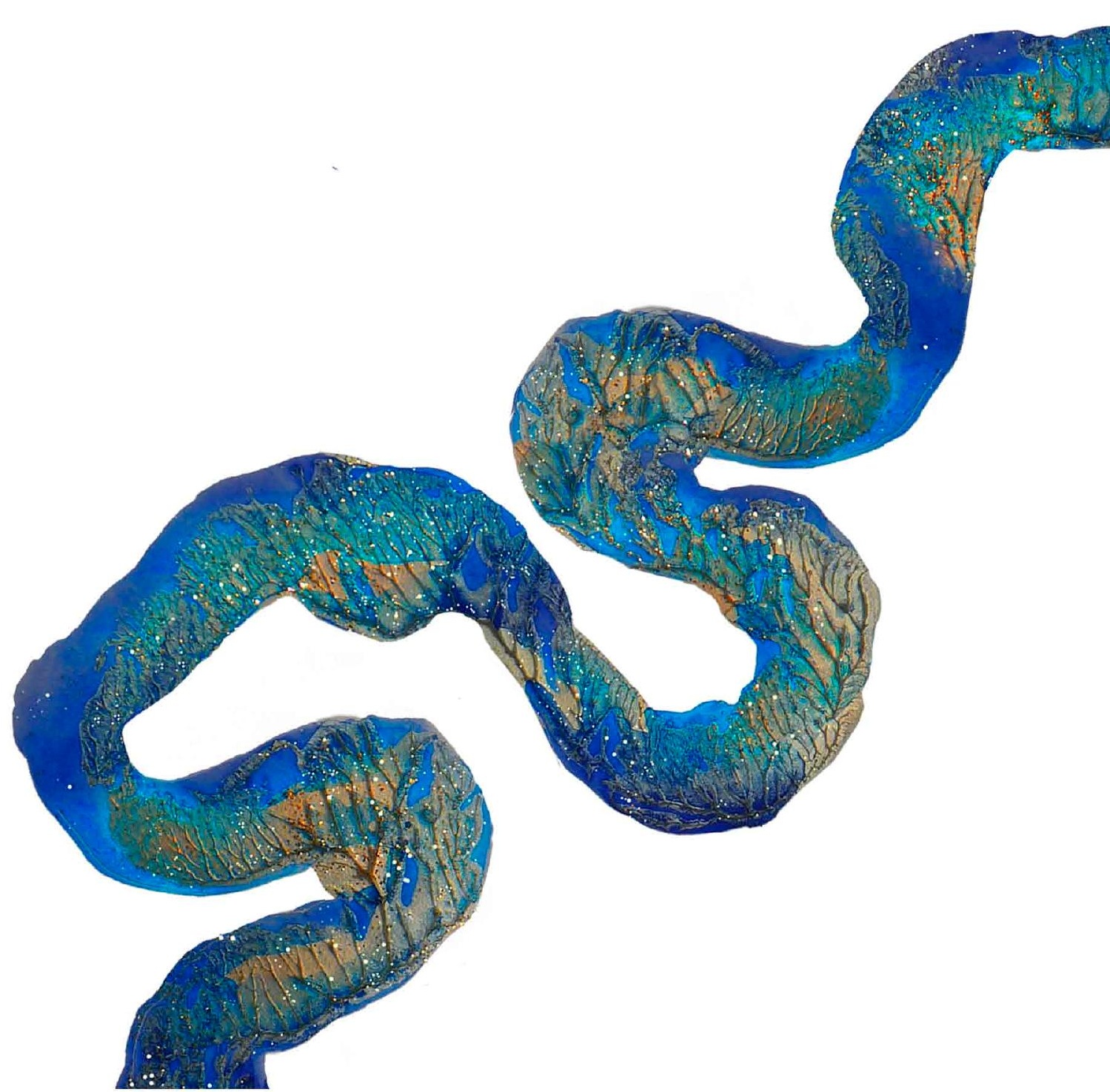




\section{SUMMARY}

The gut microbiome has become a burgeoning field of research and is proven to play a role in in many host physiological aspects, also contributing to the blood metabolome that is otherwise produced by tissues of the host organism. In order to increase the mechanistic understanding of how the microbiome influences its host health, there is a need to integrate knowledge of the composition of the gut microbiome with its functionality in terms of microbiota-mediated metabolic processes. The aim of this project was to obtain detailed insight in the mammalian-microbiome co-metabolism of endogenous metabolites and the extent to which the microbiome influences the plasma metabolome observed. This was achieved with the help of the MetaMap ${ }^{\circledR}$ Tox database.

The crosstalk between microbiome and host is often based on signal molecules or metabolites (in this context natural constituents of cells or biofluids) which can affect the host, for example via nuclear receptors leading to changes in signaling pathways, e.g. in energy metabolism or regulation of the immune system. The first stage of the investigations was the identification of microbiota-derived metabolites in plasma of male Wistar rats via treatment with antibiotics from different classes known to induce a shift of the intestinal microbiota (Chapter 2). Three specific plasma metabolome patterns for respectively the class of aminoglycosides, fluoroquinolones and tetracyclines could be established in the metabolome database called MetaMap ${ }^{\circledR}$ Tox, and plasma metabolites related to changes of the gut microbiome's functionality due to antibiotic treatment were identified. Besides metabolites such as amino acids and amino acid related metabolites, hippuric acid, indole3 -acetic acid and glycerol appeared to be microbiome-derived or -associated plasma metabolites. Indole-3-propionic acid was found to be a unique biomarker plasma metabolite for the aminoglycosides and 3-indoxylsulfate for the tetracyclines. Other plasma metabolites such as coenzyme Q, glutamine, glutamate, putrescine and spermidine were found to be mammalian-microbiome co-metabolites.

The subsequent approach entailed the metabolite profiling of different matrices of the gut of rats after treatment with antibiotics from different classes (vancomycin, streptomycin and roxithromycin) in order to evaluate metabolite changes observed at different dose levels and in male and female rats, and to identify the best tissue matrix for further investigations (Chapter 3). The same set of plasma metabolites measured in Chapter 2, with the exception of steroids and catecholamines, was also measured in the different gut matrices. The effects of the three antibiotics, each possessing a different activity spectrum, could be separated from each other in feces and cecum content, and in line with its broad spectrum of activity, vancomycin showed the most profound effect on the metabolite profile, followed by roxithromycin and then by streptomycin. Largest changes were observed for the classes of lipids, bile acids and amino acids. There were only minimal, if any, differences between males and females regarding the main metabolites driving the separation in a principal component analysis. After vancomycin treatment, a dose- 
dependent change in metabolites could be observed in feces and cecum content. Whereas in gut tissue no obvious treatment-related effect could be observed, the metabolic profiling in feces and cecum content demonstrated clear effects, allowing for an assessment to what extent the gut microbiota is affected by antibiotica. It could also be shown that metabolome changes can be detected equally well in feces as in the cecum content, thus allowing to use a non invasive method for measurements of studies on metabolism by the gut microbiota.

To gain a more comprehensive understanding of the effects and dynamics of microbiome alterations, we performed a metabolite profiling in both plasma and feces of rats after lincosamides treatment, another class of antibiotics, as well as a community analysis to characterize changes in the gut microbiome following treatment (Chapter 4). Additionally, the potential impact of the vehicle control carboxymethylcellulose (CMC) was investigated. A specific metabolite pattern could be established in the MetaMap ${ }^{\mathbb{B}}$ Tox database and further key metabolites indicating gut microbial changes could be identified or existing key metabolites could be consolidated. Most changes were observed in metabolites belonging to the class of bile acids, complex lipids, fatty acids and related metabolites, as well as amino acids and related metabolites. The diversity of the community showed no sex-specific differences and was drastically narrowed down after lincosamides treatment. Also, the vehicle control CMC showed negligible effects on both the metabolome and microbial communities.

Since the three primary bile acids which were analyzed in the metabolite profiling turned out to be consistent key metabolites indicating gut microbial changes, 20 primary and secondary bile acids were analyzed in plasma and feces of Wistar rats treated with six different antibiotics from five different classes (Chapter 5). The antibiotics had a profound impact on the bile acid metabolism via harming the intestinal microbiota - in particular shown by the poorly or non-bioavailable antibiotics streptomycin and vancomycin - and via potential feedback mechanisms regarding the bile acid production in the liver. In general, the effect of the antibiotic treatment was stronger in the feces than in plasma. Although different classes of antibiotics with different activity spectra against gut microbes were applied, they all induced an increase of taurine-conjugated primary bile acids in both plasma and feces. Contrary, cholic acid and most of the secondary bile acids were significantly downregulated in plasma whereas it accumulated in the feces. This accumulation was not seen for roxithromycin indicating a different mode of action for the macrolide antibiotic. This evaluation of changes in the plasma and feces bile acid pool helped to gain a more comprehensive understanding of how microbial changes can influence the bile acid metabolism and availability. Thus, antibiotic treatment is likely to have an influence on bioavailability of compounds and food constituents depending on bile acids for their uptake. In addition, bile acids play a role as signaling molecules. Thus, the consequences of bile acid changes for the host merit further investigations. 
The aim of this thesis was to assess functional microbial changes of the gut microbiome and to elucidate interactions between gut microbes and the host by applying metabolomics and taxonomic profiling. The results of this thesis suggest that plasma and feces based metabolic profiling via a targeted analysis turned out to be a suitable tool to investigate the microbial functionality of the gut microbiome. However, limitations emerged such as the potential systemic toxicity of the bioavailable antibiotics tested or confounders of the qualitative 16S rRNA analysis, which were discussed and future research directions were suggested in Chapter 6. In the future, alternative methods to animal studies are needed to be developed to assess, compare and to finally extrapolate the metabolic capacity of the gut microbiome from in vitro to in vivo data which is of direct relevance for pharmacology and toxicology. 


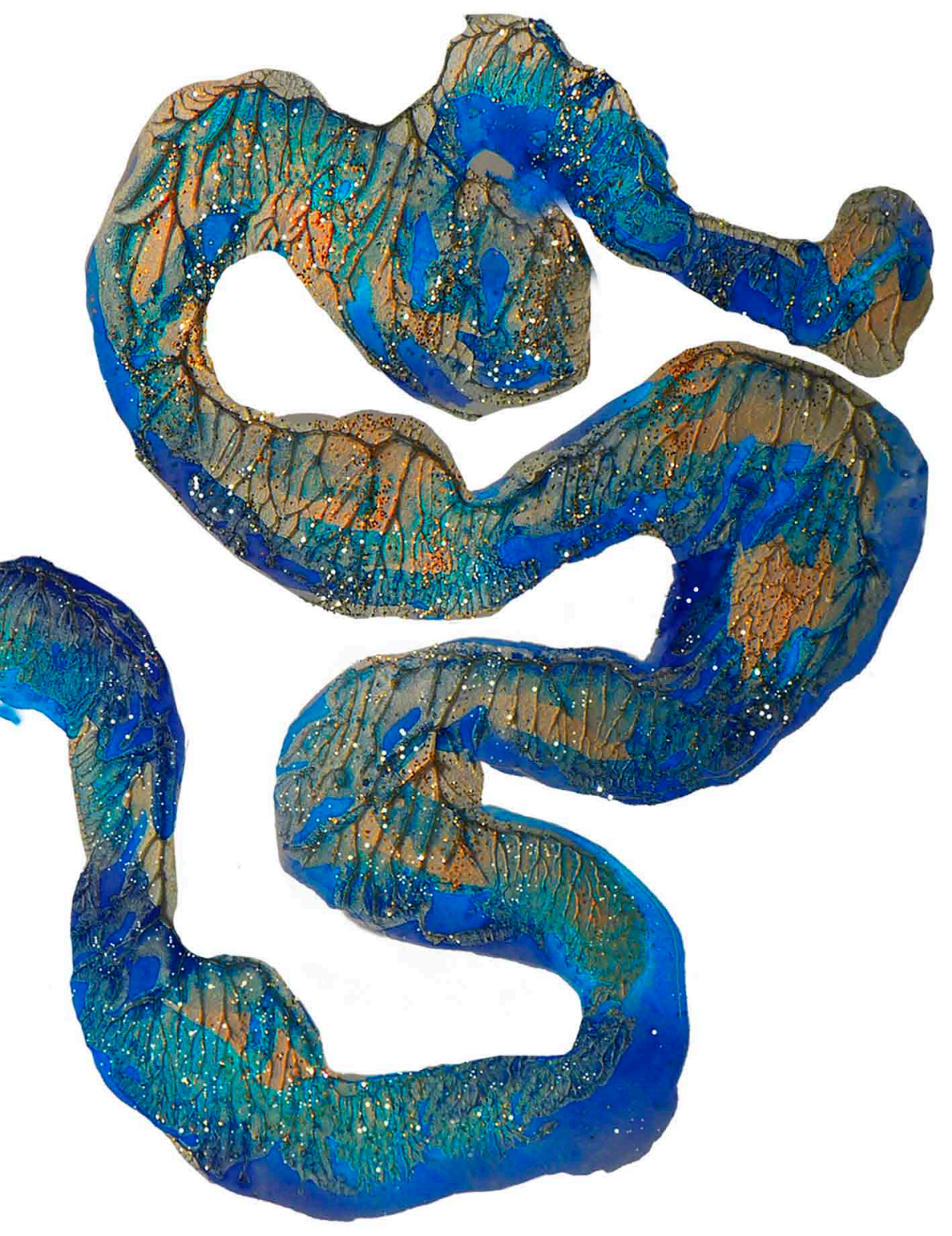




\section{Appendix}

Acknowledgements, Curriculum Vitae, List of publications, Overview of completed training activities 



\section{ACKNOWLEDGEMENTS}

First of all, I want to thank my promoter Ben. You already supervised my master thesis years ago and since then I have been continuously learning from you. With you I could vigorously discuss scientific and non-scientific topics and opinions, philosophize about life, laugh about the same jokes others couldn't understand, fool around, and celebrate successes. You always supported me and I don't want to miss you in the future. I gratefully want to thank my promoter Ivonne, for your guidance, reliability and thriving suggestions throughout the project. I am proud for having been your $\mathrm{PhD}$ student. Karsten, with you as my co-promotor, it couldn't have been better for me. Thanks for all the fruitful meetings, constructive discussions, and your sincere cordiality.

I want to thank all my co-authors and the metabolome team (special thanks to Saskia, Volker S., Eric, Barbara, Werner, Hennicke, Tilmann, Micha S., Hunter, and Volker H.). It was an outstanding chance to work with you, and in the three years of my PhD project I have learned incredibly much, both scientifically and humanly. This experience has influenced and shaped my personality, and has proved invaluable for me and the next steps of life. A special thank goes to Nicole D., who had an important role in the successful outcome of the project: you introduced me to your microbiology lab and techniques, discussed with me reasonable methods for my project, and always provided me with good advice.

Big thanks to the committee members for evaluating my thesis!

A big part of the work was also performed by my colleagues from the mechanistic toxicology (special thanks to Martina, Claudia and Burkhard) and pathology (special thanks to Frauke, Marco and Peter). Marco and Peter, I also want to thank you for all the cheerful moments and delighting coffee breaks, and of course for giving me the majestic name "Kackequeen" and the appropriate crown, which I both carry with biggest honor. Many thanks also go to the team of the clinical chemistry, first and foremost, to Irmgard: you were always there for me, thanks for your never-ending patience. Thanks Caro, for your unswerving optimism, and Gareth, thanks for your fine humor, your kind-hearted nature, and of course for the self-made scones (for which I had been waiting impatiently for years); Mrs Blum, thanks for being the good fairy of the department, for having always an open ear, the right words and free space on your couch. A big thank to all my BASF colleagues for the nice time, enjoyable talks, and your help: Gaby, Dieter D., Uwe, Frank, Markus, Thomas, Berta, Ante, Martina D., Diana\&Peter, Naveed, Mr Landsiedel, Björn, Dieter B., Kathi M., Mrs Bächle, Volker S., Lukas, Susanne, Basak, Anna T., Dani and Felix K..

An everlasting source of joy was my cozy office, namely Café UT, and this was due to all my colleagues and our awesome "neighbors" who accompanied me over the three years: first of all Xiaoqi and Johanna; Flora, Christian\&Lisa, Daniel, Juliane, Sabina, Elodie, Dunja, Eleonora, Veronique, Svenja and Jessica; Stephan, Martina and Beate. We shared uncountable special and funny moments, mountains of sweets, and developed crazy ideas 
(e.g. THE Taxi company). I found support and comprehension, and we had smart solutions for almost any problem. I also want to thank the guests of Café UT from WUR: Maria, Annelies, Mengying and Katja, who delighted me with joy and laughter - also at every visit in Wageningen, as well as my colleagues from WUR, especially Mark, Laura, Lidy and Nacho.

My deep gratitude goes to my paranymphs Laura and Johanna who both played key roles before and during my $\mathrm{PhD}$ time. Laura, I met you when I started my master thesis at the WUR tox department, and from then on, I profited from your good mood and advice, care, cordiality and creative madness. Johanna, you were my PhD student buddy and it felt we complemented each other in a mutual way. Thanks for your sensitive handling with my fears ("Midpoint"!) and your encouraging words.

Personally and outside of work, the last years have been very tough and I had to cope and struggle with many difficulties. I would not stand at the very good point where I am now without my noble friends and family. Chris, Max and my third sister Serpil, you are the persons who make me feel at home here, always being on my side in all walks of life. Meike, Ina, Xiaoqi, Georg, Yaschar, Nico, Lük, Dirk and Christoph, I want to thank you for your help, advice, humor and, above all, creating the wonderful moments of the lightness of being. Konstantin, I want to thank you for the last years. Without you, my crucial and so essential change of mind-set would not have started. Lastly, I want to thank my parents and my beloved siblings Antje, Nico and Caro for your never-ending and wholehearted support in every sense, and simply for being my family. 


\section{Curriculum Vitae}

Christina Behr was born on 14 July 1985 in Heidelberg, Germany. During her Bachelor studies at HS Mannheim, Germany, she conducted the practical semester at the Fraunhofer Institute for Interfacial Engineering and Biotechnology, Stuttgart, Germany, and graduated with the Bachelor's thesis performed at the Helmholtz-Zentrum Geesthacht, Germany, in 2012. Subsequently, she started the Master program in Toxicology at University of Kaiserslautern, Germany, and spent six months at the Division of Toxicology, Wageningen University, in cooperation with BASF SE, Germany, for MSc graduation. In 2015, Christina started her $\mathrm{PhD}$ study as an external $\mathrm{PhD}$ student at the Department of Experimental Toxicology and Ecology, BASF SE, in Ludwigshafen and the Division of Toxicology, Wageningen University, under the supervision of Prof. Bennard van Ravenzwaay and Prof. Ivonne Rietjens, as well as Dr. Karsten Beekmann. During her PhD, she followed a postgraduate education program in Toxicology, which enables to register as "Fachtoxikologin DGPT" and European Toxicologist. 


\section{LIST OF PUBLICATIONS}

Behr, C., Kamp, H., Fabian, E., Krennrich, G., Mellert, W., Peter, E., Strauss, V., Walk, T., Rietjens, I.M.C.M., van Ravenzwaay, B., 2017. Gut microbiome-related metabolic changes in plasma of antibiotic-treated rats. Arch. Toxicol. 91, 3439-3454. https://doi.org/10.1007/s00204-017-1949-2

Behr, C., Sperber, S., Jiang, X., Strauss, V., Kamp, H., Walk, T., Herold, M., Beekmann, K., Rietjens, I.M.C.M., van Ravenzwaay, B., 2018b. Microbiome-related metabolite changes in gut tissue, cecum content and feces of rats treated with antibiotics. Toxicol. Appl. Pharmacol. 355, 198-210. https://doi.org/10.1016/j.taap.2018.06.028

Behr, C., Ramírez-Hincapié, S., Cameron, H.J., Strauss, V., Walk, T., Herold, M., Beekmann, K., Rietjens, I.M.C.M., van Ravenzwaay, B., 2018a. Impact of lincosamides antibiotics on the composition of the rat gut microbiota and the metabolite profile of plasma and feces. Toxicol. Lett. 296, 139-151. https://doi.org/10.1016/j.toxlet.2018.08.002

Behr, C., Slopianka, M., Haake, V., Strauss, V., Sperber, S., Kamp, H., Walk, T., Beekmann, K., Rietjens, I.M.C.M., van Ravenzwaay, B., 2019. Analysis of metabolome changes in the bile acid pool in feces and plasma of antibiotic-treated rats. Toxicol. Appl. Pharmacol. 363, 79-87. https://doi.org/10.1016/j.taap.2018.11.012 
OVERVIEW OF TRAINING ACTIVITIES

Discipline specific courses (DGPT courses)

Molecular Toxicology

Metabolism of xenobiotics and toxicokinetics

Risk assessment

Organ Toxicology

Ecotoxicology
Munich, Germany

2016

Zurich, Switzerland

2016

Berlin, Germany

2017

Erfurt, Germany

2017

Schmallenberg, Germany

\section{Meetings and conferences}

German Pharm-Tox Summit,

Berlin, Germany

2016

Poster presentation

German Pharm-Tox Summit,

Poster presentation

Eurotox meeting,

Heidelberg, Germany

2017

Oral presentation

Heidelberg Forum for Young Life Scientists

German Pharm-Tox Summit,

Bratislava, Slovakia

2017

Heidelberg, Germany

2017

Göttingen, Germany

2018

Oral and poster presentation

Annual Meeting of the Society of Toxicology, San Antonio, USA

2018

Oral and poster presentation

\section{General courses and optional activities}

Preparation of $\mathrm{PhD}$ research proposal

BASF Tox, Germany

2015

Introduction course to $\mathrm{R}$

University of Mannheim, 2017

Germany

Preparation of LRI research proposal

BASF Tox, Germany

2018

Attending scientific presentations

BASF Tox, Germany

2015-2018

General Toxicology 


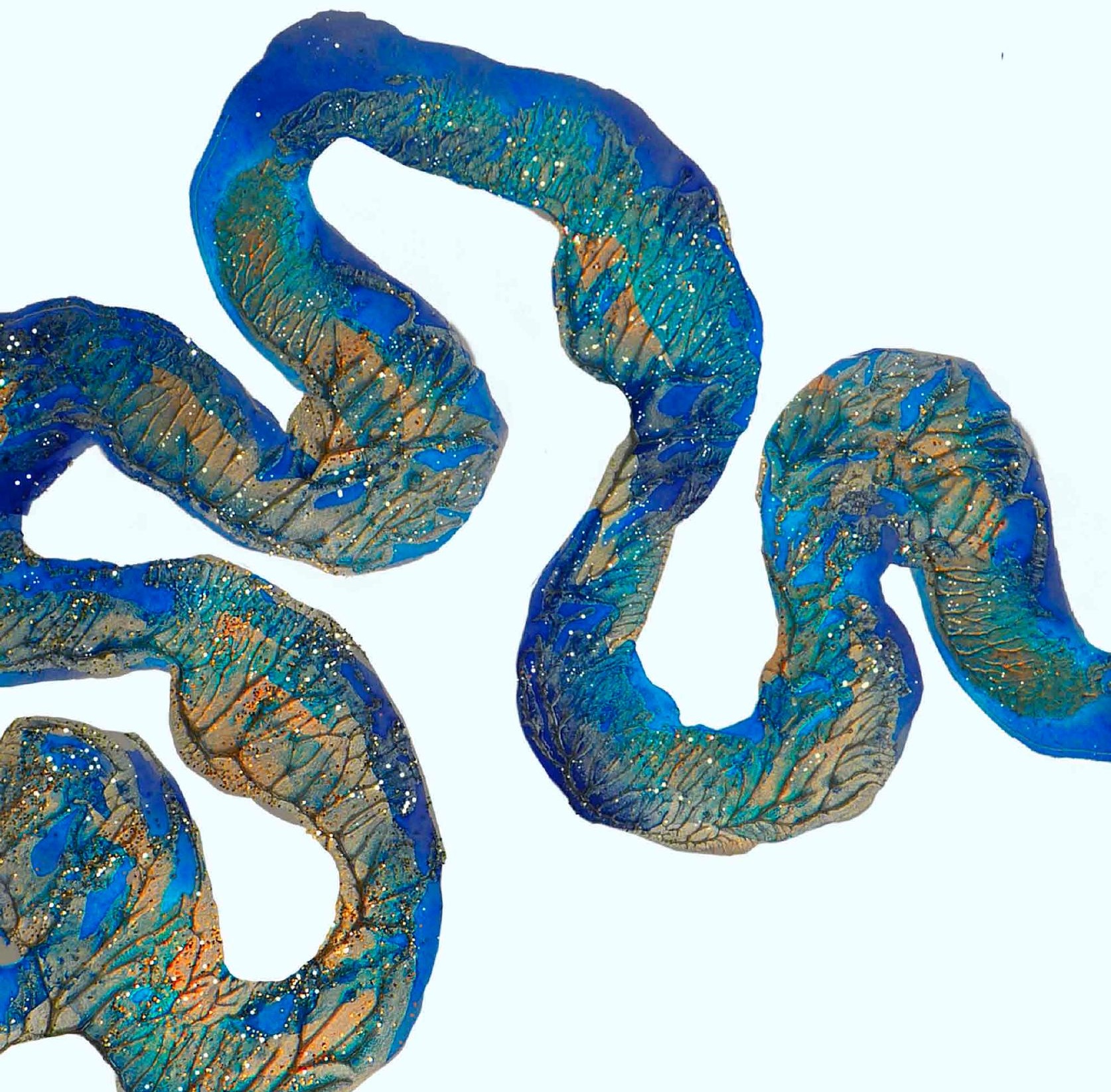


The research described in this thesis was financed by BASF SE, Ludwigshafen, Germany.

Financial support from Wageningen University for printing this thesis is gratefully acknowledged.

Cover \& Layout design by Carolin Behr and Antje Behr

Printed by Digiforce || Proefschriftmaken.nl, Vianen, the Netherlands 